Draft Final Technical Report • January 2002

\title{
INITIAL DEVELOPMENT OF A CONTINUOUS EMISSION MONITOR FOR DIOXINS
}

Final Technical Report

Reporting Period Start Date:July 29, 1998

Reporting Period End Date: January 31, 2002

Authors:

Michael J. Coggiola, Harald Oser, Gregory W. Faris, and David R. Crosley

Report Issue Date: January 2002

DOE Award Number: $\quad$ DE-AC26-98FT-40370

Prepared by: $\quad$ SRI International

333 Ravenswood Avenue

Menlo Park, CA 94025-3493

Prepared for: $\quad$ U.S. Department of Energy

National Energy Technology Laboratory

3610 Collins Ferry Road

Morgantown, WV 26505

\begin{abstract}
This report was prepared as an account of work sponsored by an agency of the United States Government. Neither the United States Government nor any agency thereof, nor any of their employees, makes any warranty, express or implied, or assumes any legal liability or responsibility for the accuracy, completeness, or usefulness of any information, apparatus, product, or process disclosed, or represents that its use would not infringe privately owned rights. Reference herein to any specific commercial product, process, or service by trade name, trademark, manufacturer, or otherwise does not necessarily constitute or imply its endorsement, recommendation, or favoring by the United States Government or any agency thereof. The views and opinions of authors expressed herein do not necessarily state or reflect those of the United States Government or any agency thereof.
\end{abstract}




\begin{abstract}
Under contract DE-AC26-98FT-40370, SRI International has completed the third phase of a planned three-phase effort to develop a laboratory prototype continuous emission monitor (CEM) for dioxins and furans generated during the incineration of waste materials at DOE remediation sites. The project was initiated on July 29, 1998 with the technical effort completed in October
\end{abstract} 2001.

During this research effort, SRI has made numerous improvements in our jet-REMPI instrument. These improvements have involved characterization and optimization of the molecular cooling in the gas jet, implementation of a custom-fabricated, four pulsed valve assembly, new data acquisition and display software, and preliminary development of a wavelength and mass calibration approach. We have also measured the REMPI excitation spectra of numerous organic compounds that are likely to be present in the exhaust stream of a waste incinerator. These spectra must be well characterized in the laboratory to understand any potential interferences that might arise when monitoring for dioxin and furan congeners.

Our results to date continue to validate the original concept of using jet-REMPI as the detection method in a dioxin CEM. Using only commercial components with minor modifications, we have already demonstrated a detection sensitivity in the low ppt range with sufficient chemical specificity to separately detect two closely related congeners of dichlorodibenzodioxin present in a mixture.

To demonstrate the utility of this methodology outside of the controlled conditions of the laboratory, we performed a series of pseudo-field experiments at the US Environmental Protection Agency's National Risk Management Research Laboratory, Research Triangle Park, NC. The instrument used for those studies was built by SRI under contract with US EPA, and was an exact duplicate of the SRI system. This duplication allowed the experiments to be conducted without transporting the SRI system to the EPA site. Using the jet-REMPI system in conjunction with a combustion flow reactor, the joint SRI-EPA team discovered several new, and unexpected, chemical species in the exhaust stream of a pure methane flame.

Based on our work in this project, we have developed a number of concepts for instrumental improvements that will substantially increase our sensitivity while maintaining the exceptional selectivity required of a dioxin CEM. In addition, we have developed several system configurations with varying degrees of functionality that can be further developed and deployed for process monitoring, surrogate measurements, and potentially, as a dioxin control CEM. Due to the extremely demanding regulatory compliance monitoring requirements involving both congener 


\section{Draft}

specificity and sub-part-per-trillion sensitivity with near real-time speed, we believe it is not as yet possible to specify a system configuration for a true dioxin compliance monitor.

While a true TEQ compliance monitor is not yet possible using the jet-REMPI approach, the technique may prove useful as a surrogate, or indicator monitor. This application would involve continuous measurement of surrogate compounds, such as lowly chlorinated dioxins and furans, whose concentrations have been previously correlated with the TEQ. Such an instrument would not require the extreme sensitivity of a compliance monitor although the high degree of chemical selectivity would remain important. 


\section{CONTENTS}

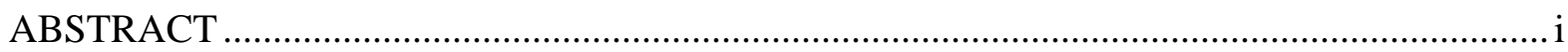

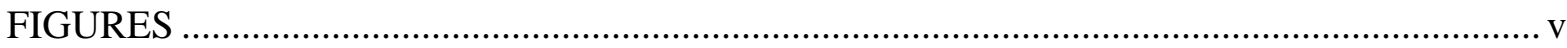

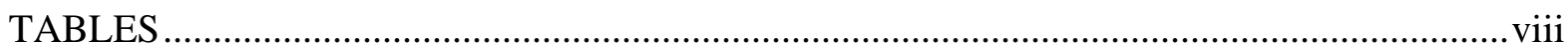

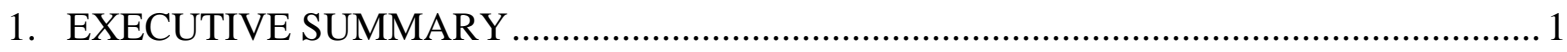

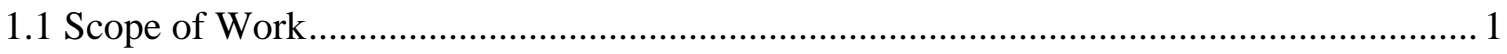

1.2 Project Objectives - Laboratory Prototype Instrument ……………….......................... 1

1.2.1 Prototype Improvement .............................................................................. 1

1.2.2 Sampling and Preconcentration.....................................................................

1.2.3 Instrument Optimization................................................................................

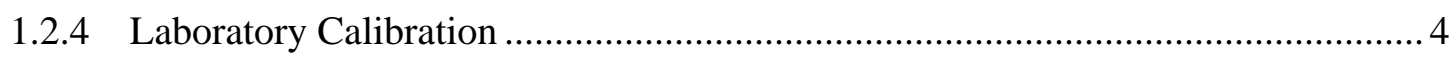

1.2.5 Dioxin CEM Design Specification ..............................................................

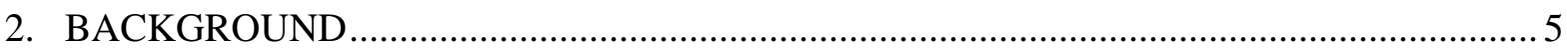

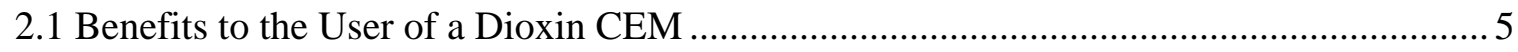

2.2 Technology Development Requirements ...................................................................

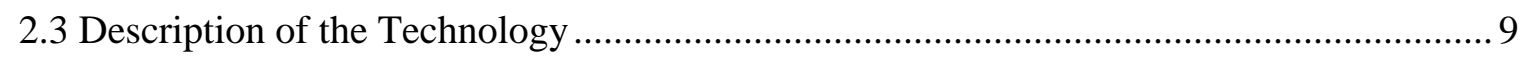

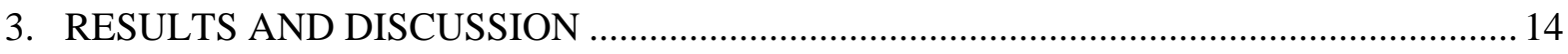

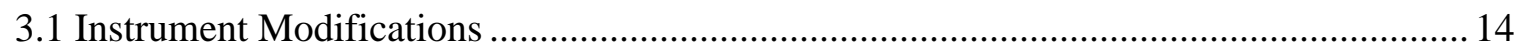

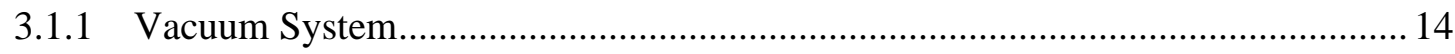

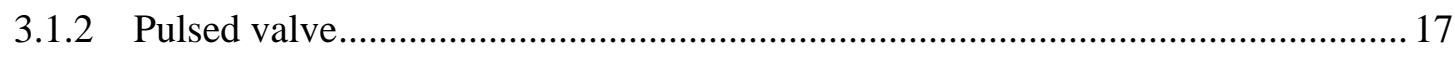

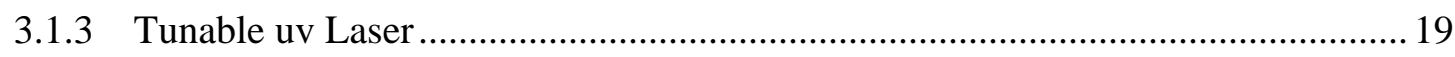

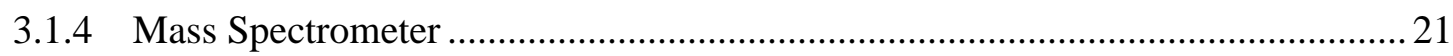

3.1.5 New Optical Frequency Doubler......................................................................2 22

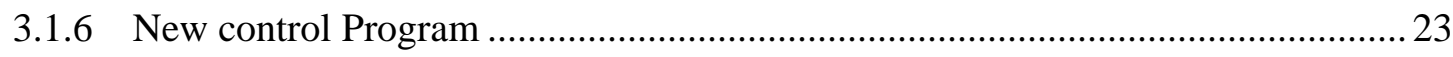

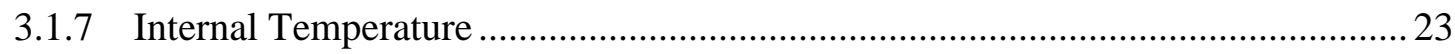

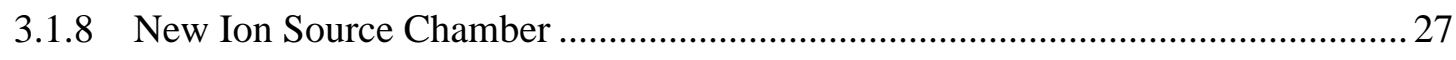

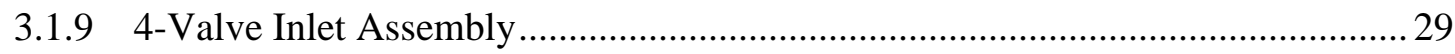

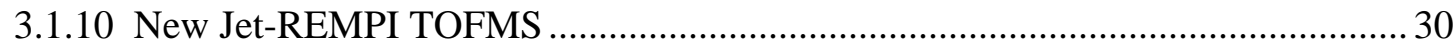

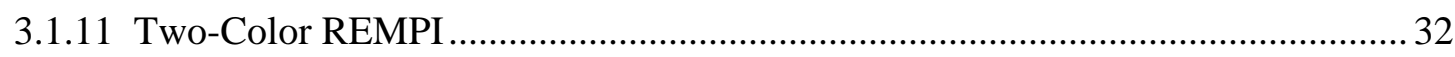

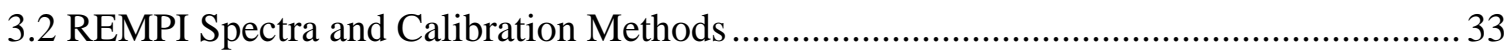

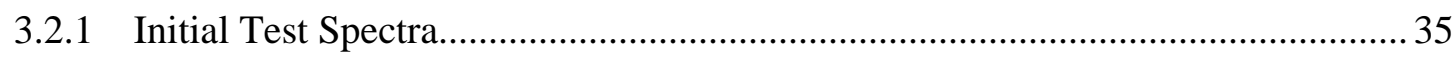

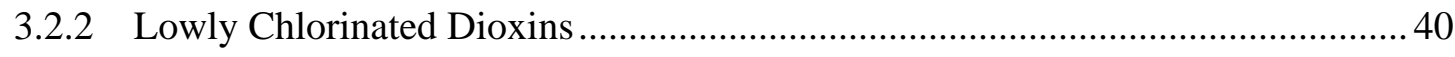

3.2.3 Other REMPI Spectra.................................................................................. 43

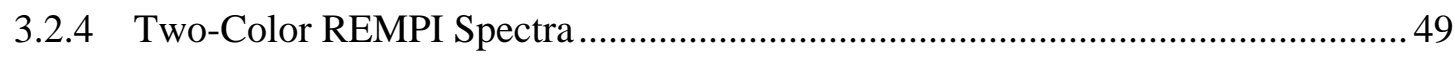

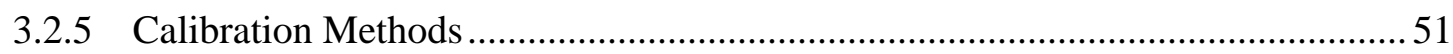




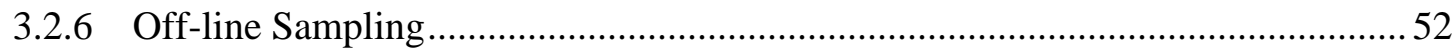

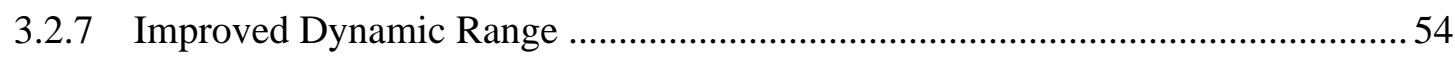

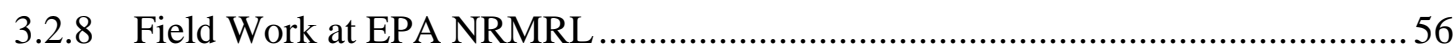

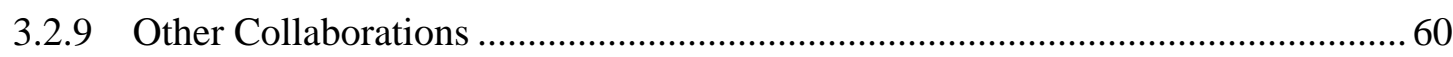

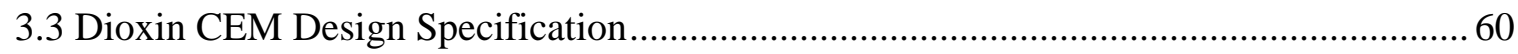

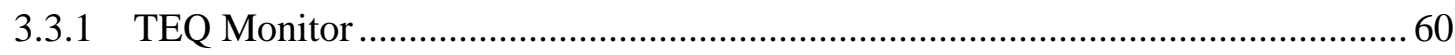

3.3.2 Alternative REMPI-Based Instrumentation Schemes ...............................................63

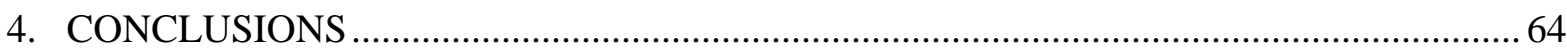

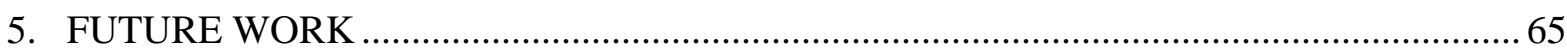

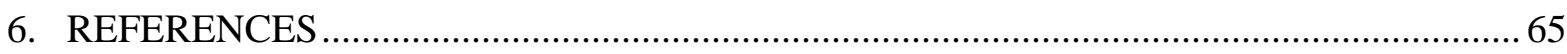

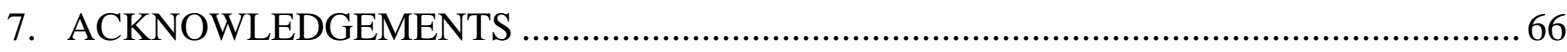

8. APPENDIX A: Preliminary Test Plan "Field Measurements Using Jet-REMPI:

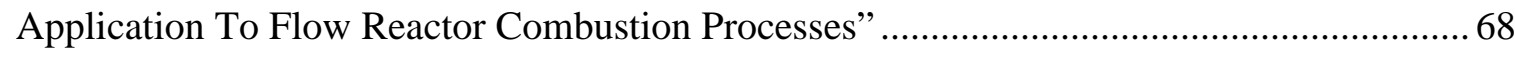

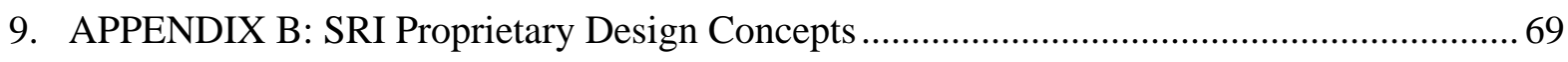

10. APPENDIX C: "Development of a jet-REMPI (resonantly enhanced multiphoton ionization) continuous monitor for environmental applications", Appl. Opt. 40, 859 (2001)..................74

11. APPENDIX D: "Congener-specific detection of dioxins using jet-REMPI", Chemosphere 43, 469 (2001). 82

12. APPENDIX E: "Development Of A Real-Time Continuous Emissions Monitor For Dioxins And Other Chlorinated Aromatics", Organohalogen Compounds 40, 551(1999). ...92

13. APPENDIX F: "Development Of A Real-Time Continuous Emissions Monitor For Hazardous Aromatic Air Pollutants", Organohalogen Compounds 45, 141(2000). 97

14. APPENDIX G: "Dioxin And Furan Laboratory Measurements Using Jet-REMPI “, Organohalogen Compounds 54, 360(2001). 102 


\section{FIGURES}

1. Two-dimensional map of the mass and wavelength space for a number of hazardous air pollutants, including lowly chlorinated dioxins. The Xs correspond to the center wavelength for the principal absorption feature for each species.

2. High resolution, two-dimensional map of the mass and wavelength space for the 2,3- and 2,8-dichlorodibenzodioxins. The approximate width of the principal absorption feature for each congener is shown by the line connecting the Xs. The instrumental mass resolution and optical resolution are shown by the vertical and horizontal bars, respectively

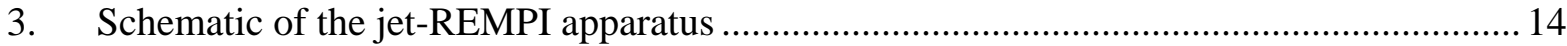

4. Design of the jet-REMPI vacuum housing............................................................... 15

5. Design of the rectangular detector port modification to the time -of-flight mass

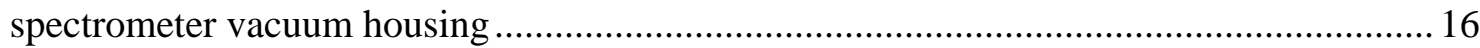

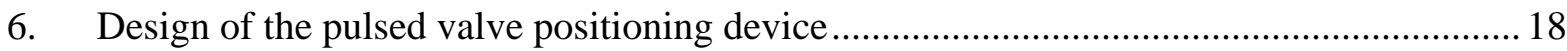

7. Schematic diagram of the valve positioning system installed on the ion source vacuum housing

8. Comparison of the measured and calculated band profiles for a valve-to-laser separation of $2.3 \mathrm{~cm}$ for a $\mathrm{S}_{1} \leftarrow \mathrm{S}_{0}$ transition in benzene.

9. Variation of the jet-REMPI signal for benzene measured at $78 \mathrm{amu}$ and a wavelength of $259.1 \mathrm{~nm}$ as a function of the laser-valve separation.

10. Comparison of the measured and calculated band profiles for a valve-to-laser separation of $7.5 \mathrm{~cm}$ for a $\mathrm{S}_{1} \leftarrow \mathrm{S}_{0}$ transition in benzene.

11. Variation of the jet-REMPI density distribution (signal level) for benzene measured at $78 \mathrm{amu}$ and a wavelength of $259.1 \mathrm{~nm}$ as a function of the delay between the valve trigger and the laser pulse. The corresponding rotational temperature as determined by fitting the band profiles is also shown.

12. Schematic of the new ion source vacuum chamber including the sliding four-valve assembly

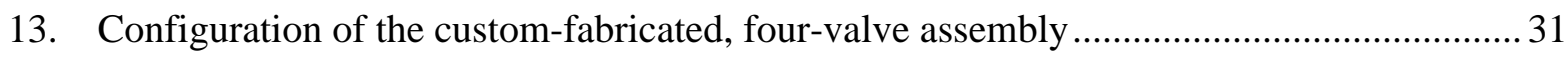

14. Cross sectional view of one valve and the base plate of the four-valve assembly ..............31

15. Photograph of the new TOFMS and existing laser system at SRI 
16. Variation of the $\mathrm{S} 0 \rightarrow \mathrm{S} 1$ excitation energy (o) as a function of chlorination for dibenzodioxins. Also shown is the one-color, two-photon ionization limit (n) corresponding to one half of the ionization potential. The S0 $\rightarrow \mathrm{S} 1$ excitation energy for the tetrachlorinated dibenzodioxin is estimated from conventional uv absorption

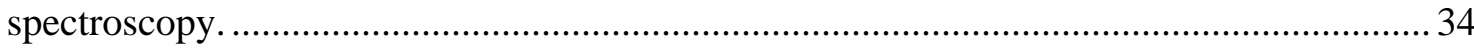

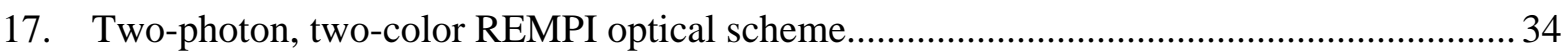

18. Mass spectrum recorded for jet-REMPI detection of 2,5-dichlorotoluene at a wavelength of $279.6 \mathrm{~nm}$. The inset shows an expanded region about the parent ion peaks. The chlorine isotope peaks are clearly resolved.

19. Wavelength dependence of the $\mathrm{m} / \mathrm{z} 160$ ion signal for jet-REMPI detection of 2,5dichlorotoluene

20. Wavelength dependence of the $\mathrm{m} / \mathrm{z} 146$ ion signal for the jet-REMPI detection of 1,2-dichlorobenzene .38

21. Wavelength dependence of the $\mathrm{m} / \mathrm{z} 112$ ion signal for the jet-REMPI detection of monochlorobenzene..

22. Mass spectrum recorded for jet-REMPI detection of 1,2-dichlorobenzene at wavelength of $261.2 \mathrm{~nm}$

23. Mass spectrum recorded for jet-REMPI detection of monochlorobenzene at wavelength of $266.0 \mathrm{~nm}$

24. Wavelength dependence of the $\mathrm{m} / \mathrm{z} 218$ ion signal for the jet-REMPI detection of 2monochlorodibenzodioxin

25. Wavelength dependence of the $\mathrm{m} / \mathrm{z} 252$ ion signal for jet-REMPI detection of 2,7dichlorodibenzodioxin

26. Wavelength dependence of the $\mathrm{m} / \mathrm{z} 252$ ion signal for jet-REMPI detection of 2,8dichlorodibenzodioxin

27. Wavelength dependence of the ion signal at $\mathrm{m} / \mathrm{z} 252$ for the jet-REMPI detection of a mixture of 2,7-dichlorodibenzodioxin and 2,8-dichlorodibenzodioxin.

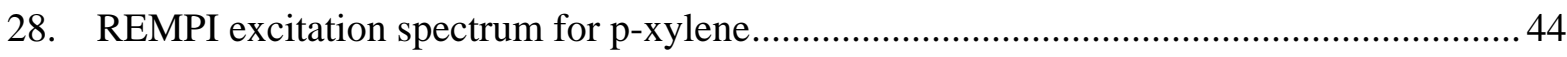

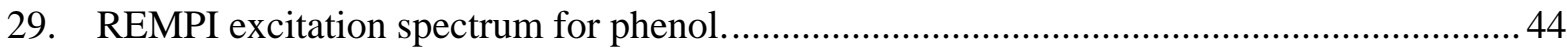

30. False color map showing the intensity (color) measured as a function of wavelength and mass for a mixture of benzene ( $78 \mathrm{amu}$ ), toluene (92 amu), and chlorobenzene (112 amu).

31. REMPI excitation spectra for benzene, toluene, and chlorobenzene derived from the three-dimensional map in Figure 30. .46

32. Wavelength dependence for the REMPI ionization of aniline 
33. Wavelength dependence for the REMPI ionization of o-toluidine.

34. Wavelength dependence for the REMPI ionization of dibenzodioxin.

35. Wavelength dependence for the REMPI ionization of 2-monochlorodibenzofuran .

36. Wavelength dependence of the ion signal at $\mathrm{m} / \mathrm{z} 146$ for the jet-REMPI detection of 1,2-dichlorobenzene. The lower trace was recorded using a one-color, two-photon, REMPI scheme. The upper trace was recorded using a two-color, two-photon REMPI scheme where the second color was at a fixed wavelength of $266 \mathrm{~nm}$.

37. Dependence of the REMPI ionization signal for 2-monochlorodibenzofuran using different excitation schemes

38. REMPI excitation spectra for a 1:1 mixture of benzene (78 amu) and perdeuterobenzene ( $84 \mathrm{amu}$ ) recorded simultaneously as a function of REMPI excitation wavelength.

39. Test of dual digitizer data acquisition system. The upper trace was acquired on the first digitizer with a full scale input of $0.237 \mathrm{Vdc}$. The center trace was acquired on the second digitizer with a full scale input of $1.282 \mathrm{Vdc}$, and the lower trace is the composite signal produced according to the algorithm described in the text.

40. Diagram of the US EAP combustion research flow reactor used in the field measurements 57

41. Time dependence of phenol measured in a lean methane flame at EPA ..........................58

42. Time dependence of phenol measured in a lean methane flame at EPA ...........................59

43. Wavelength dependence of the aniline jet-REMPI signal measured in a lean methane flame at EPA 


\section{TABLES}

1. Hazardous air pollutants previously measured by jet-REMPI. ........................................ 12

2. Performance specifications of the Continuum laser system.............................................. 20

3. Comparison between required and currently available performance specifications

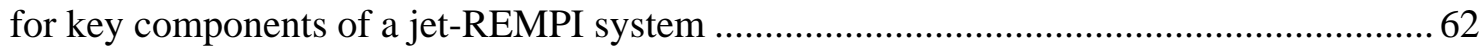




\section{EXECUTIVE SUMMARY}

Under contract DE-AC26-98FT-40370, SRI International has completed all three phases of a planned three-phase effort to develop a laboratory prototype continuous emission monitor (CEM) for dioxins and furans generated during the incineration of waste materials at DOE remediation sites. During the 42 month course of executing this project, some of the originally proposed tasks were either modified, deleted, or replaced to reflect the changing state of knowledge, the technical progress, unresolved technical issues, and unplanned, new research opportunities and directions.

\subsection{SCOPE OF WORK}

The objective of this effort is to develop a laboratory instrument that can provide continuous monitoring of the emission levels of polychlorinated dibenzo-p-dioxins (PCDDs) and polychlorinated dibenzofurans (PCDFs) generated by incineration equipment at DOE sites. Once developed, this instrument will be used to systematically study the emission levels of key dioxins and furans that contribute to the toxic equivalence (TEQ). This information, combined with mechanistic modeling studies being undertaken elsewhere, will lead to the design specifications for a real-time, autonomous dioxin CEM that can be used for compliance monitoring at DOE incinerators.

The goal of the first phase of the effort was to establish the sensitivity and chemical specificity using currently available dioxin detection instrumentation. The instrument developed in Phase 1 required a stepwise process of designing, testing, evaluating, and upgrading that was continued in Phase 2. Phase 2 included extensive, systematic testing of the laboratory prototype, performance evaluation, and a design for upgrading to a system that can measure each of the dioxin and furan congeners that contribute to the TEQ. Phase 3 included additional instrumental improvements, expansion of the spectral library, and testing the laboratory system under simulated field conditions of a sample stream containing typical levels of flue-gas combustion by-products.

\subsection{PROJECT OBJECTIVES - LABORATORY PROTOTYPE INSTRUMENT}

\subsubsection{Prototype Improvement}

A major objective of our effort has been to make incremental improvements in the analytical capabilities of the jet-REMPI system. Initial evaluations of the sensitivity and specificity for the baseline instrument assembled during Phase 1 were used to establish needed changes and to refine the laboratory dioxin CEM system. These tasks helped to establish which 
chemical species can be monitored by a prototype instrument and how best to optimize the ionization scheme and laser source(s), the mass spectrometer configuration, and numerous other design and operational parameters.

SRI successfully modified an existing TOFMS for use as the laboratory prototype dioxin CEM. All the planned major modifications were completed, as well as several additional, unplanned modifications that became necessary during the work. At the completion of Phase 1, the SRI instrument was performing as well or better than any jet-REMPI instrument reported in the open scientific literature.

SRI made considerable progress on the improvement of the operation of our prototype jet-REMPI apparatus. These improvements are described in detail below in Section 3 of this report. At the conclusion of this effort, the complete system, including the tunable laser and the mass spectrometer were fully functional. The overall reliability, stability, and reproducibility of the instrument were greatly improved.

\subsubsection{Sampling and Preconcentration}

The goal of this task was to address the critical issues associated with acquiring a representative sample of the stack emissions and transporting that sample to the instrument without significant loss or change in its chemical composition. Because the sensitivity of the instrument remains significantly below that required for a dioxin CEM compliance monitor, some form of sample preconcentrator may need to be developed.

SRI made relatively little progress on this task for a variety of reasons. The issue of sampling is closely tied to the specific details of the source from which the sample is to be extracted. Because we as yet have no definite test bed with which to test our system, it is difficult to design a universal sampler. Similarly, the design of a preconcentrator, or even the need for a preconcentrator, cannot be established until the instrumental sensitivity has been maximized to the extent possible. Since we are continuing to improve the limits of detection through optimization of the instrument, we cannot as yet determine if preconcentration will be required. Moreover, we have become aware of a preliminary preconcentrator design being developed by the research group of Dr. Brian Gullett, of the National Risk Management Research Laboratory, U.S. Environmental Protection Agency.

We have collaborated with this group on a number of dioxin CEM issues, including the design of a preconcentrator. At the conclusion of the present effort, the EPA had assembled a prototype preconcentrator, but no testing had been completed.

Additionally, we have also become aware of other DoE-funded work on sampling and preconcentration being performed at both MSE Technology Applications, Inc. (MSE), Butte, MT, and the Diagnostic Instrumentation \& Analysis Laboratory (DIAL), at Mississippi State 
University, Mississippi State, MS. SRI has maintained contact with researchers at both institutions, as well as making on-site visits to view and discuss their sampling designs.

\subsubsection{Instrument Optimization}

SRI successfully investigated the sensitivity of the jet-REMPI instrument using several lowly chlorinated dioxin congeners. Detailed "calibration curves" were not generated, however, because the focus was on estimating the limit of detection (LOD) rather than on establishing a useful range of linear response. In addition, each of many of the improvements made on a continuing basis would necessitate repeated remeasuring of the calibration curves. Since other groups have demonstrated linear response over five to six orders of magnitude using a very similar jet-REMPI instrument, it is reasonable to assume that our instrument will provide at least equivalent performance.

In an extension of the proposed sensitivity testing, SRI was able to demonstrate two critical additional performance characteristics: (1) congener-specific detection in mixtures and (2) two-photon, two-color REMPI detection of a chlorinated aromatic compound. Both of these performance characteristics are essential for a dioxin CEM.

This task was designed to investigate the function of key elements of the instrument, including the pulsed gas inlet valve, laser ionization source(s), ion extraction optics, and the mass spectrometer. The commercial, pulsed, inlet gas valve used initially may eventually be replaced with one designed specifically for this application. Design criteria will include operation at elevated temperatures, repetition rate and pulse length commensurate with the laser ionization source(s), and a geometry that maximizes the sample gas density in the ionization region.

The use of two-color REMPI schemes were also studied as a means of improving both the sensitivity and the chemical selectivity. The ion extraction optics were carefully designed to take into account four factors: (1) the field distortions associated with the proximity of the pulsed gas valve to the ionization region, (2) the geometry of the ionization region, (3) space charge effects within the extraction volume, and (4) the phase space volume associated with the ion acceptance of the mass spectrometer. Computer simulations using SIMION 6.0 were used extensively for this design.

A systematic study of the supersonic molecular beam formed by the free jet expansion was performed. This characterization allowed SRI to optimize the location of the ionization region with respect to the exit of the nozzle. This optimization not only increased the sensitivity of the instrument by maximizing the ionization yield, but it also allowed for an improved and simplified mechanical design of the source vacuum chamber. 
Finally, the functioning of the mass spectrometer was investigated with respect to its transmission, resolution, sensitivity, and background noise level.

\subsubsection{Laboratory Calibration}

The goal of this task was the calibration of the laboratory prototype instrument to establish its sensitivity, chemical specificity, response time, measurement reproducibility, and reliability. Using the optimized instrument developed in Phase 2, SRI originally planned a series of comprehensive laboratory tests using a combination of PCDDs and PCDFs that contribute to the TEQ . The bulk of these laboratory tests were replaced with a pseudo-field test performed at EPA's National Risk Management Research Laboratory (NRMRL), Research Triangle Park, NC.

The vapor delivery system that would have been used for the calibration studies proved more difficult to design and construct than originally planned due to the strict materials limitations (for example, no Teflon or other polymeric seals), and the necessity of operating at elevated temperatures. We originally had planned to use standard, commercial permeation devices, however, these were not readily available for chlorinated dioxins and furans, nor were they designed to operate above $70^{\circ} \mathrm{C}$. Because of these restrictions, we had to develop a design that did not use normal valves and seals which could result in loss of the target compounds due to adsorption or chemical degradation. Our design required the fabrication of several pieces of custom glassware. Although all the necessary components were eventually acquired, we did not complete the assembly and testing, choosing instead to make the measurements at NRMRL.

SRI made the first measurements of the REMPI excitation spectrum of benzene-d $\mathrm{d}_{6}\left(\mathrm{C}_{6} \mathrm{D}_{6}\right)$ which could serve as a calibrant molecule. The use of this compound as both a mass and wavelength calibration standard was demonstrated. Although more work will be required to implement an automated calibration scheme based on perdeuterobenzene, our preliminary experiments show that it is feasible to do this.

\subsubsection{Dioxin CEM Design Specification}

At the conclusion of this effort, we believe that the required performance specifications of a dioxin CEM instrument capable of serving as a regulatory monitor are beyond the current state of the technology. Nonetheless, it is possible to provide a general design specification for this instrument.

Although specific design elements can be specified, the exact laser or TOFMS configuration can change in time not only because many choices and combinations are possible, but also because both components are continually being improved by commercial manufactures. At the current stage of development, it is much more important to establish feasibility and general design specifications, than to project specific criteria for rapidly evolving technologies, such as laser sources. 
We provide a general design specification in Section 3.3, along with a discussion of the current state of technological development for the key components. Finally, we provide a discussion of optional monitoring systems that are possible using the REMPI TOFMS approach.

\section{BACKGROUND}

Current techniques for monitoring emissions of PCDDs and PCDFs use sampling times in excess of hours, during which the analytes are collected on adsorbing materials followed by sample extraction and preparation for later gas chromatography/mass spectrometry (GC/MS) analysis. ${ }^{1}$ These costly and time demanding methods have drawbacks in that compliance measurements are made only infrequently (perhaps once or twice per year). The consequences are overdesigned air pollution control systems and regulatory strategies that rely on indirect process monitoring rather than direct monitoring and dioxin prevention strategies.

\subsection{BENEFITS TO THE USER OF A DIOXIN CEM}

In light of these limitations, a continuous emission monitor (CEM) for PCDD and PCDF offers four benefits to users:

- $\quad$ Direct, rapid detection of PCDD and PCDF congeners, their indicators (compounds measured in lieu of PCDDs and PCDFs that indicate the parallel presence of PCDDs and PCDFs), or their precursors (compounds that have been shown to be chemical progenitors of PCDDs and PCDFs).

- $\quad$ Combustion system optimization through continuous, on-line monitoring and process control.

- A method to advance prevention of PCDD and PCDF formation rather than rely on flue gas cleaning controls.

- Assurance to stakeholders (such as permit writers and the public) that the process is operating safely.

The U.S. EPA Office of Solid Waste (OSW), which regulates hazardous waste treatment processes, has identified continuous PCDD and PCDF monitoring as a research priority, because PCDDs and PCDFs can drive risk assessments. ${ }^{2}$ The OSW recognizes that CEMs offer superior continuous compliance assurance compared with infrequent, extractive sampling. Their policy provides the economic incentive for waste facilities to use CEMs by eliminating waste feed characterization, compliance testing, and operating parameter monitoring for pollutants when a 
facility uses CEMs. However, with few PCDD/PCDF CEM technologies under development, the DOE will not be able to implement this superior mode of compliance. A CEM for compliance purposes will likely require higher performance capabilities (especially for sensitivity) than if the CEM were used for research purposes or as a method of combustion optimization. These CEM sensitivity needs will be lessened by using a short duration sample concentration method. However, sensitivity for a dioxin CEM is a primary issue, requiring measurements at concentrations two to four orders of magnitude lower than ever achieved before.

As suggested by experts in this area, ${ }^{3}$ a dioxin CEM should first be used as a research tool in laboratories studying PCDD and PCDF formation and control. Such an instrument will need to make rapid, accurate measurements of PCDDs and PCDFs but at concentrations much higher than needed for a compliance CEM. This type of instrument will greatly accelerate our understanding of PCDD and PCDF formation and the availability of prevention and control techniques. Researchers have limited understanding of how combustion processes affect PCDD and PCDF formation, largely due to their need to relate time-integrated sampling data with dynamic formation mechanisms and combustor conditions after an often multi-week analysis lag period. A real-time CEM will provide immediate feedback on how variations in combustion operating parameters affect PCDD and PCDF formation and/or destruction, thus allowing more accurate correlations and much more comprehensive data analysis. An instrument that can make these types of measurements will also be valuable to DOE as new waste treatment processes are evaluated and readied for permitting and public acceptance.

As our understanding of PCDD and PCDF formation improves, it will be valuable to build a database using emissions from actual waste treatment processes to correlate operating conditions with PCDD and PCDF formation. Such a database can be used to devise operating strategies to prevent formation of PCDD and PCDF. This database can also be used to identify surrogates or indicators that can be monitored more easily and cheaper than the PCDD and PCDF themselves, leading to less expensive, more widely implemented compliance and control strategies.

CEMs also provide data important for stakeholder's assurance that the combustion processes are operating safely. Stakeholders such as public interest groups, permit writers, and local citizens groups can play a major role in permitting waste treatment facilities. Real-time emissions data may accelerate their acceptance, saving time and money during the permitting process. 
This overall approach of coordinating dioxin measurement and control extends beyond the scope of this project and will involve several government agencies. SRI is closely involved with the dioxin monitoring community and we are working to bring this broader vision to reality. This project focuses on the issue of dioxin measurement and how to develop a REMPI (resonance enhanced multiphoton ionization) -based CEM system to measure the type and concentrations of dioxins necessary to provide DOE a means to continuously monitor dioxins for regulatory compliance.

Two critical issues must be addressed before a dioxin CEM can be successfully developed:

- What would you measure? There are 210 PCDD and PCDF congeners, with widely varying levels of toxicity, and most are not considered toxicologically significant.

- To what minimum concentration (sensitivity) would you need to measure it? The draft MACT rule proposes an emission limit of $0.2 \mathrm{ng} / \mathrm{m}^{3}$ 2,3,7,8 TCDD toxic equivalence (TEQ).

Toxic equivalence factors (TEF) are defined as a set of weighting factors expressing the toxicity of each compound relative to the toxicity of 2,3,7,8 TCDD. TEFs vary from 1.0 to 0.001 for 17 different 2,3,7,8-substituted compounds. The total TEQ is calculated by multiplying the concentration of each PCDD and PCDF by its corresponding factor TEF. Several weighting schemes are available. NATO has proposed a scheme assigning factors to 17 different 2,3,7,8substituted compounds and assigning no factor to the 193 non-2,3,7,8-substituted compounds. Estimates of performance needs for a dioxin CEM for regulatory compliance can be made based on the existing knowledge of TEF and TEQ. Considering only the 17 compounds in the total TEQ, the sensitivity required for a dioxin CEM would need to be a factor of ten less than the emission limit for each of the 17 congeners (i.e., divide the TEQ by 17 x 10). Hence, the required minimum detection limit is equivalent to the emission limit divided by 170 , or 0.001 $\mathrm{ng} / \mathrm{m}^{3}$ or better, for each congener in the TEQ.

In the best case, if you could argue that the probability of formation of each congener was about equal, then the minimum detection limits could be higher for compounds with a lower TEQ factor. In other words, given a minimum detection limit of $0.001 \mathrm{ng} / \mathrm{m}^{3}$ for 2,3,7,8 TCDD, the other $16 \mathrm{PCDD} / \mathrm{F}$ congeners having TEFs between 0.5 to 0.001 would have corresponding minimum detection limits of $0.002 \mathrm{ng} / \mathrm{m}^{3}$ to $1.0 \mathrm{ng} / \mathrm{m}^{3}$.

As shown in the estimates above, the detection limits must be very low to directly measure all 17 congeners in the TEQ. A proposed alternative is to monitor precursors or 
surrogates, identified using a dioxin CEM in research laboratories to collect real-time data. This real-time data would be invaluable to develop mechanistic understandings, leading to surrogates or precursors that can be measured at much higher concentrations. According to experts in this area, ${ }^{4}$ the study of lightly chlorinated (mono-, di-, or tri-) dioxins would be acceptable, because these congeners are easier to measure. The molecules are very similar to those in the TEQ and would likely lead to correlations describing the formation of TEQ congeners.

Laboratory experiments for studying dioxin formation and control usually operate at total dioxin concentrations around 100 to $1000 \mathrm{ng} / \mathrm{m}^{3}$. Depending on how many of the 210 total congeners are present, detection limits around $5 \mathrm{ng} / \mathrm{m}^{3}$ for each congener of interest would be more than enough. The first step in developing a dioxin CEM suitable for compliance monitoring is, therefore, to develop a laboratory instrument capable of speciating dioxin congeners, precursors, or surrogates, and measuring their concentrations in real-time with a sensitivity in the low $\mathrm{ng} / \mathrm{m}^{3}$ range. By combining this information with sophisticated combustion models, it will be possible to determine the specific target chemicals that must be measured by a dioxin CEM, and the levels to which they must be measured. Only after this information is available can a dioxin CEM suitable for compliance use be developed.

In recent years, many significant improvements have been made in the detection of dioxins resulting from various combustion processes. Several research groups, including DLR (Deutsche Forschungsanstalt für Luft und Raumfahrt) in Stuttgart, Germany, ${ }^{3,5}$ The Technical University of Munich, Germany, ${ }^{6,7}$ U.S. university research at Ann Arbor and Cornell, and Sandia National Laboratories ${ }^{8}$ in Livermore, CA, have demonstrated instruments or components of instruments based on REMPI. To date, however, none of these research programs has achieved the sensitivity required for either compliance monitoring or a research CEM to study formation and control of dioxins. The possibility of a commercial dioxin CEM becoming available within the next five to ten years is questionable without a development program focused on achieving specific performance goals for a dioxin CEM.

Of particular interest and promise for the real-time characterization of dioxins is the combination of a pulsed gas jet with REMPI and time-of-flight mass spectrometry (TOFMS). Using this approach, the group at DLR Stuttgart has measured non-, mono-, di-, tri-, and tetrachlorinated dioxins at the EPA National Risk Management Laboratory (NRML) facility during tests from July to October, 1996. ${ }^{9}$ The measured minimum detectability for dichlorinated dioxins was approximately $20 \mathrm{ng} / \mathrm{m}^{3}$. We estimate that this detection limit can be improved by at least one order of magnitude to about $1 \mathrm{ng} / \mathrm{m}^{3}$ for dichlorinated dioxins. Using the current onecolor REMPI scheme, the sensitivity decreases with increasing chlorination, making detection of the more highly chlorinated dioxins problematic. 


\subsection{TECHNOLOGY DEVELOPMENT REQUIREMENTS}

REMPI needs significant enhancements to reach the sub-ng/ $\mathrm{m}^{3}$ detection limits required for a compliance CEM. Potential enhancements include the use of sample preconcentration, a two-color REMPI scheme, and higher repetition rate laser systems. As a research dioxin CEM, however, the current REMPI-TOFMS scheme may need to be improved by only one or two orders of magnitude.

Based on our previous research with REMPI and our understanding of dioxin regulatory structure, four major areas must be studied to develop a REMPI-based dioxin CEM:

- Determination if penta- and higher chlorinated dioxins will require a twocolor REMPI scheme.

- Measurement of detection limits for congeners as a function of chlorination and improvement in the detection limits via system improvements and sample preconcentration.

- Development of a particle desorber/separator to liberate dioxins adsorbed on particles for detection by the REMPI analyzer.

- Development of a mechanistic understanding of dioxin formation and control to determine which congeners should be measured, and at what concentration, to provide measurements for regulatory compliance and process control.

This project will address the first three areas and provide the tools necessary to address the fourth. We continue to work closely with dioxin research groups to ensure that the dioxin mechanistic studies are integrated into the instrument development work.

\subsection{DESCRIPTION OF THE TECHNOLOGY}

Resonance enhanced multiphoton ionization is a highly sensitive, highly species-selective, gas-phase analysis technique that has been applied to numerous problems in molecular spectroscopy and combustion research. SRI has been using multiphoton-mass spectrometric techniques to study spectroscopy and trace-level detection for more than a decade. We pioneered the application of these methods to the detection of aromatic and chlorinated organic compounds. ${ }^{10,11}$ For REMPI of complex molecules, such as dioxins, the spectra can be simplified greatly by expansion through a nozzle. Adiabatic expansion results in low sample temperatures, which increases the electronic ground state population and narrows the resonance line widths. The enhanced population of the ground state gives an increase in sensitivity, while the narrower line 
widths give rise to very sharp REMPI transitions. One or two lasers are used to ionize the cooled gas molecules by absorption of two or more photons, one of which is resonant with an electronic transition in the target molecule.

Ions produced by REMPI are often detected using a TOFMS that takes advantage of the pulsed nature and well-defined temporal character of laser ionization. Many REMPI experiments performed at SRI have successfully used the TOFMS approach. The simultaneous detection by mass and wavelength yields high chemical selectivity. Typical sensitivities of conventional REMPI TOFMS systems that do not use sample molecule cooling are in the $\mathrm{mg} / \mathrm{m}^{3}$ range and higher, which is insufficient either for a laboratory system or for regulatory monitoring of chlorinated aromatics in a waste treatment process such as an incinerator.

A major improvement in sensitivity without loss in selectivity can be achieved using a pulsed gas valve that produces a supersonic expansion. In a supersonic jet, the temperature drop occurs only in a relatively narrow zone downstream of the nozzle but upstream of the Mach disk, or shock front. Laser ionization in the region of the Mach disk provides the highest sensitivity due to the local maximum in gas density. This phenomenon was exploited by SRI in a 1987 study of the four-photon dissociation and ionization of $\mathrm{H}_{2}$ in a pulsed jet. ${ }^{12}$

The combination of REMPI and mass spectrometry provides a unique, two-dimensional, approach to the analytical measurement of trace levels of organic compounds in complex mixtures. The two-dimensional nature of REMPI-MS is illustrated in Figure 1, which compares 28 hazardous air pollutants (HAPs) by plotting their molecular weight as a function of the primary $\mathrm{S}_{0} \rightarrow \mathrm{S}_{1}$ transition wavelength. The molecular weight corresponds to mass that would be monitored by the MS, while the transition wavelength corresponds to the optical wavelength used to resonantly excite the molecule. It is apparent that, for these selected HAPs, closely related species tend to cluster together, as might be expected.

At first glance, it appears, however, as if some species may not be distinguishable. For example, in the upper right corner, the 2,3-dichlorodibenzodioxin and the 2,8dichlorodibenzodioxin are nearly coincident on this map. In actuality, these species are readily separable using jet-REMPI, as shown in the high resolution, two-dimensional map in Figure 2. Four dioxin species fall within the narrow mass and wavelength ranges displayed in Figure 2, corresponding to the 2,3- and 2,8-dichlorodibenzodioxin molecules containing two chlorine-35 atoms, and one chlorine- 35 and one chlorine- 37 atom. On the expanded scale, the $S_{0} \rightarrow S_{1}$ transition wavelength is seen to actually have a finite width, typically $0.03 \mathrm{~nm}$, as shown by the horizontal bars for each species. The four molecules are widely separated on this map compared with our instrumental resolution, both in mass (approximately $0.25 \mathrm{amu}$ ) and wavelength 
(approximately $0.005 \mathrm{~nm}$ ). Hence, jet-REMPI TOFMS can easily distinguish these four species. This capability was verified experimentally in Phase 1.

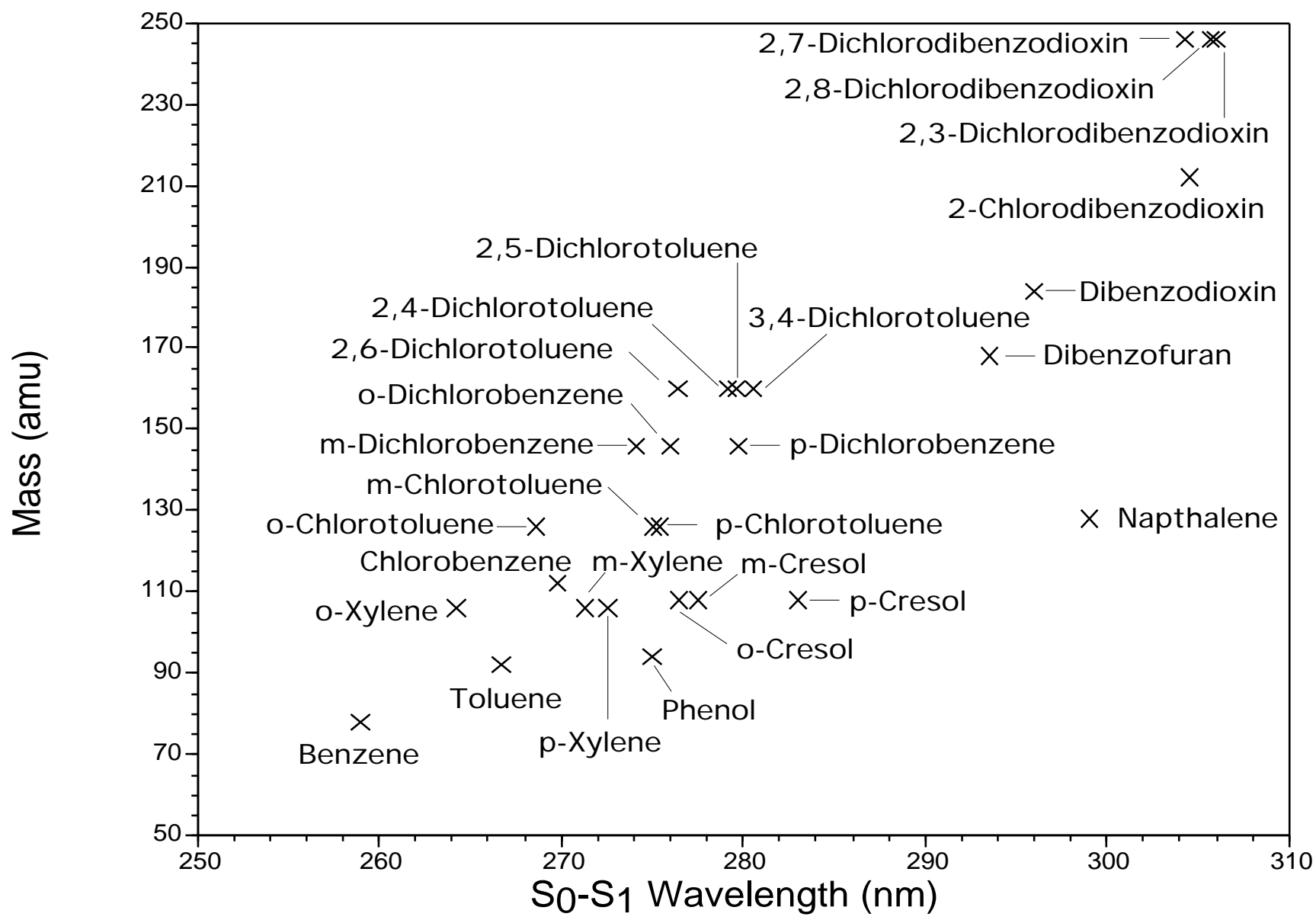

Figure 1. Two-dimensional map of the mass and wavelength space for a number of hazardous air pollutants, including lowly chlorinated dioxins. The Xs correspond to the center wavelength for the principal absorption feature for each species.

Table I lists the pertinent information for a range of hazardous air pollutants that have been previously examined using jet-REMPI. This list is not intended to be comprehensive but rather illustrative of the general classes of compounds that readily accessible using this vapor phase method. 


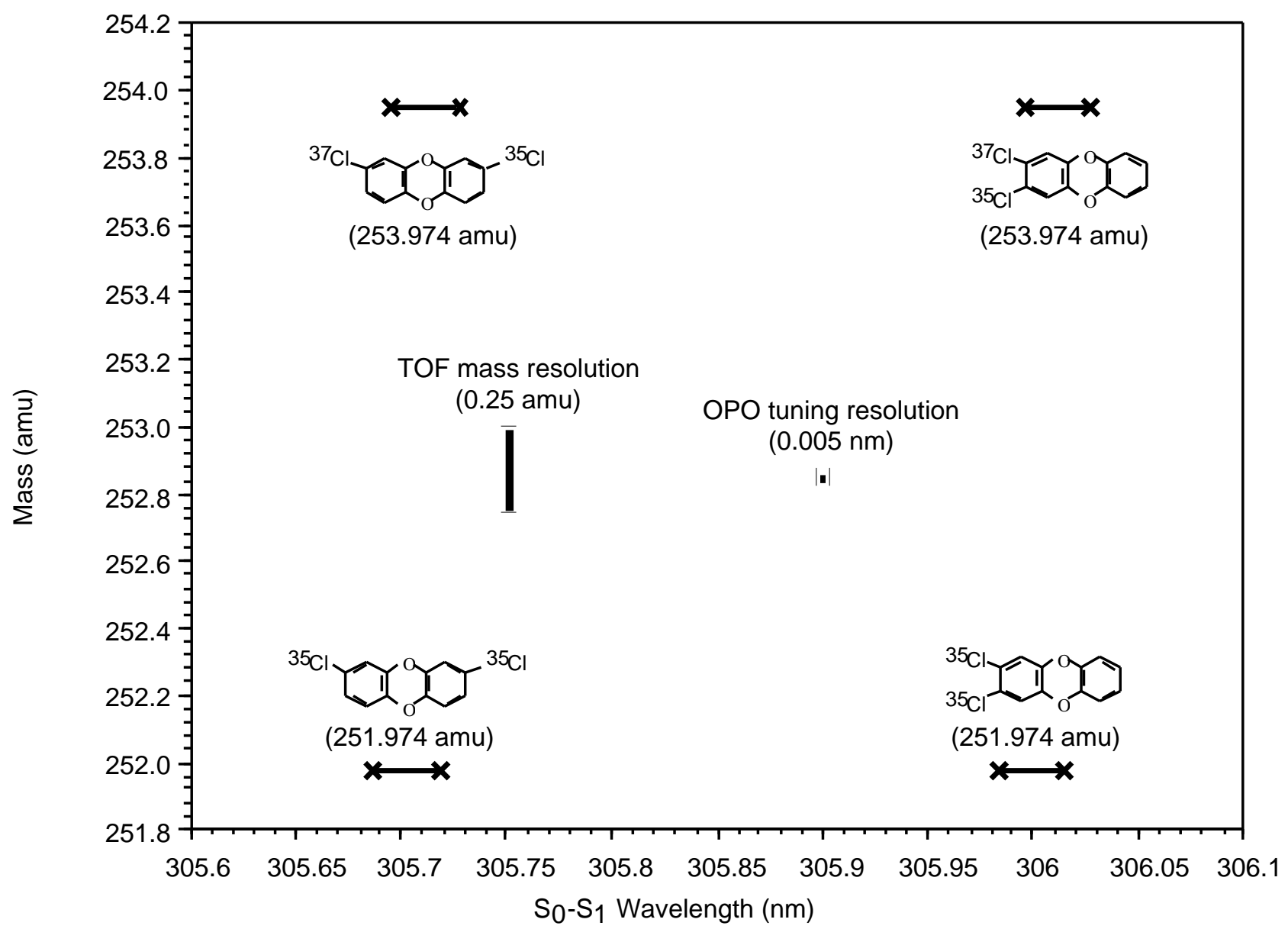

Figure 2. High resolution, two-dimensional map of the mass and wavelength space for the 2,3- and 2,8-dichlorodibenzodioxins. The approximate width of the principal absorption feature for each congener is shown by the line connecting the Xs. The instrumental mass resolution and optical resolution are shown by the vertical and horizontal bars, respectively.

Table 1

HAZARDOUS AIR POLLUTANTS PREVIOUSLY MEASURED BY JET-REMPI

\begin{tabular}{|c|c|c|c|}
\hline Molecule & Formula & Mass [amu] & $\underline{S}_{0}-S_{1}[\mathrm{~nm}]$ \\
\hline Benzene & $\mathrm{C}_{6} \mathrm{H}_{6}$ & 78 & 259.0 \\
\hline Toluene & $\mathrm{C}_{7} \mathrm{H}_{6}$ & 92 & 266.7 \\
\hline Phenol & $\mathrm{C}_{6} \mathrm{H}_{6} \mathrm{O}$ & 94 & 275.0 \\
\hline o-Xylene & $\mathrm{C}_{8} \mathrm{H}_{10}$ & 106 & 261.8 \\
\hline m-Xylene & $\mathrm{C}_{8} \mathrm{H}_{10}$ & 106 & 260.0 \\
\hline p-Xylene & $\mathrm{C}_{8} \mathrm{H}_{10}$ & 106 & 259.7 \\
\hline o-Cresol & $\mathrm{C}_{7} \mathrm{H}_{8} \mathrm{O}$ & 108 & 276.5 \\
\hline
\end{tabular}




\begin{tabular}{lccc} 
m-Cresol & $\mathrm{C}_{7} \mathrm{H}_{8} \mathrm{O}$ & 108 & 277.5 \\
p-Cresol & $\mathrm{C}_{7} \mathrm{H}_{8} \mathrm{O}$ & 108 & 283.0 \\
Chlorobenzene & $\mathrm{C}_{6} \mathrm{H}_{5} \mathrm{Cl}$ & 112 & 269.8 \\
o-Chlorotoluene & $\mathrm{C}_{7} \mathrm{H}_{7} \mathrm{Cl}$ & 126 & 268.6 \\
m-Chlorotoluene & $\mathrm{C}_{7} \mathrm{H}_{7} \mathrm{Cl}$ & 126 & 275.1 \\
p-Chlorotoluene & $\mathrm{C}_{7} \mathrm{H}_{7} \mathrm{Cl}$ & 275.4 \\
Naphthalene & $\mathrm{C}_{10} \mathrm{H}_{8}$ & 126 & 299.0 \\
o-Dichlorobenzene & $\mathrm{C}_{6} \mathrm{H}_{4} \mathrm{Cl}_{2}$ & 128 & 276.0 \\
m-Dichlorobenzene & $\mathrm{C}_{6} \mathrm{H}_{4} \mathrm{Cl}_{2}$ & 146 & 274.1 \\
p-Dichlorobenzene & $\mathrm{C}_{6} \mathrm{H}_{4} \mathrm{Cl}_{2}$ & 146 & 279.7 \\
2,4-Dichlorotoluene & $\mathrm{C}_{7} \mathrm{H}_{6} \mathrm{Cl}_{2}$ & 146 & 279.2 \\
2,5-Dichlorotoluene & $\mathrm{C}_{7} \mathrm{H}_{6} \mathrm{Cl}_{2}$ & 160 & 279.6 \\
2,6-Dichlorotoluene & $\mathrm{C}_{7} \mathrm{H}_{6} \mathrm{Cl}_{2}$ & 160 & 276.4 \\
3,4-Dichlorotoluene & $\mathrm{C}_{7} \mathrm{H}_{6} \mathrm{Cl}_{2}$ & 160 & 280.6 \\
Dibenzofuran & $\mathrm{C}_{12} \mathrm{H}_{8} \mathrm{O}$ & 160 & 293.5 \\
Dibenzodioxin & $\mathrm{C}_{12} \mathrm{H}_{8} \mathrm{O}_{2}$ & 168 & 296.0 \\
2-Chlorodibenzodioxin & $\mathrm{C}_{12} \mathrm{H}_{7} \mathrm{ClO}_{2}$ & 184 & 305.7 \\
2,3-Dichlorodibenzodioxin & $\mathrm{C}_{12} \mathrm{H}_{6} \mathrm{O}_{2} \mathrm{Cl}_{2}$ & 212 & 306.0 \\
2,7-Dichlorodibenzodioxin & $\mathrm{C}_{12} \mathrm{H}_{6} \mathrm{O}_{2} \mathrm{Cl}_{2}$ & 246 & 246.3 \\
2,8-Dichlorodibenzodioxin & $\mathrm{C}_{12} \mathrm{H}_{6} \mathrm{O}_{2} \mathrm{Cl}_{2}$ & 246 & \\
\hline & & & 304.5 \\
\hline & & 246.0 & 2 \\
\hline
\end{tabular}

Additional experimental details on the method and data acquired during the course of this project are described in two recent publications, included in Appendix C: "Development of a jetREMPI (resonantly enhanced multiphoton ionization) continuous monitor for environmental applications", Appl. Opt. 40, 859 (2001), and Appendix D: "Congener-specific detection of dioxins using jet-REMPI", Chemosphere 43, 469 (2001). SRI's contributions to the 1999, 2000, and 2001 annual dioxin conferences are also included as Appendices E-G, respectively. 


\section{RESULTS AND DISCUSSION}

\subsection{INSTRUMENT MODIFICATIONS}

Figure 3 is a schematic of the laboratory prototype REMPI TOFMS instrument we assembled for this effort. The TOFMS was originally developed for NASA to provide real-time chemical characterization of tropospheric aerosol particles. That instrument, with a suitable aerosol inlet stage, was successfully deployed on a NASA DC-8 research aircraft as part of a two-month field mission. Described below are numerous modifications, improvements, and additions made in the original TOFMS system for this project.

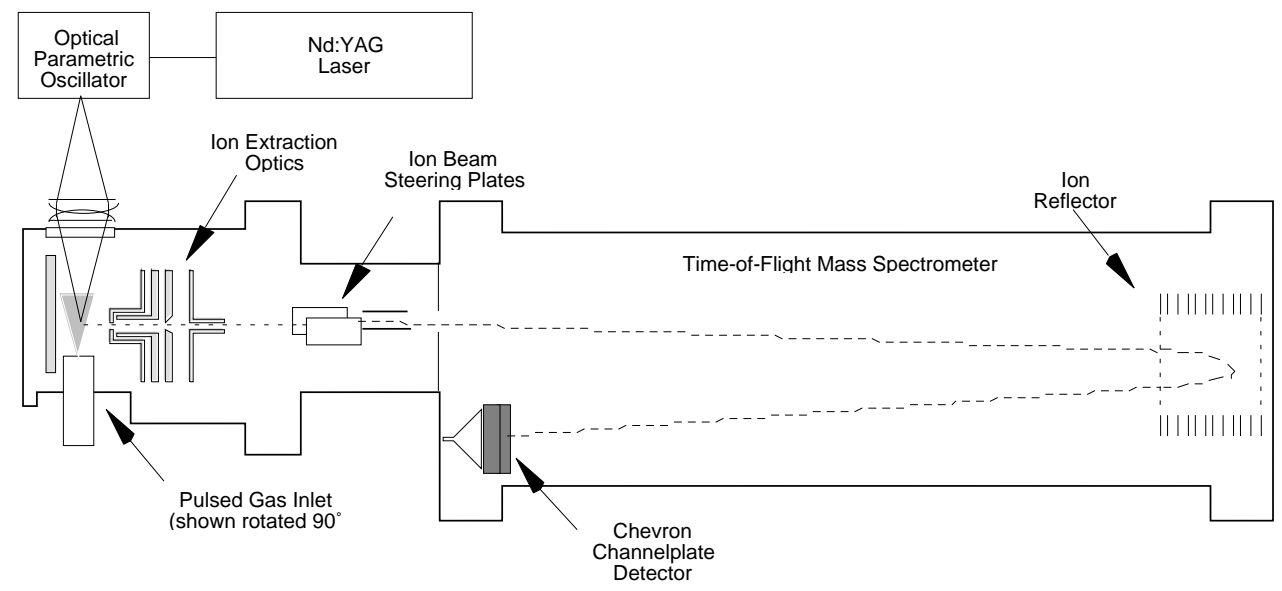

Figure 3. Schematic of the jet-REMPI apparatus.

\subsubsection{Vacuum System}

The first step in this project was to modify of our existing time-of-flight mass spectrometer system. To use the system for the jet-REMPI application, a new source vacuum housing was required. The new source chamber included two ports for laser windows, large diameter ports for the pulsed valve, the turbomolecular pump and connection to the flight tube, and three smaller ports for pressure gauges and other feedthroughs. Figure 4 shows a sketch of the chamber fabricated from a commercial, 8-inch diameter, stainless steel "tee" manufactured by MDC Vacuum Products, Hayward, CA. A still newer source chamber was fabricated later in the project as described in Section 3.1.8. 


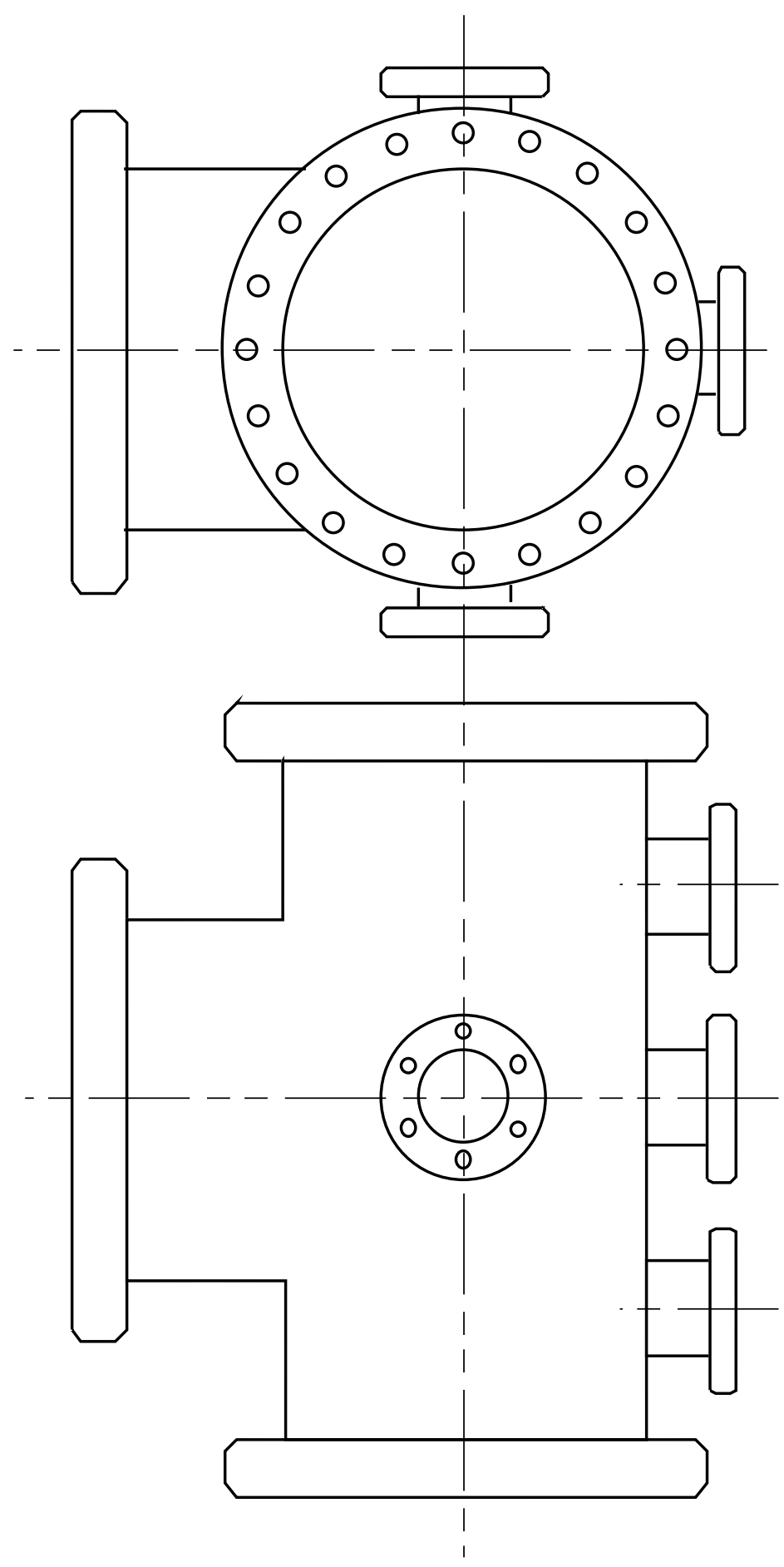

Figure 4. Design of the jet-REMPI vacuum housing.

In parallel with fabrication of the new source housing, we also modified the main vacuum chamber of the TOF flight tube to permit ready access to the microchannel plate detector assembly. The commercial vacuum chamber as supplied by R. M. Jordan, Grass Valley, CA, 
requires a significant degree of disassembly to access the detector. Under normal conditions, this is not a problem because the detector is relatively robust. However, our experience with this instrument indicates that the detector produces an unacceptably large amount of ringing following the detection of a strong ion signal. This ringing in the signal results in loss of data for several hundred nanoseconds following the strong peak. Typically, this type of ringing indicates of an impedance mismatch somewhere in the detection circuitry. Correction of a mismatch requires a trial-and-error approach of testing different resistive termination and capacitive bypass configurations under actual detection conditions. The need to change the configuration frequently makes the rapid disassembly and reassembly of the detector important. Because of physical constraints and the design of the microchannel plate detector, we added a rectangular, metal-gasketed, flange to the side of the flight tube, as shown in Figure 5. The rectangular port permits easy removal and installation of various detector configurations without resorting to disassembly of the main vacuum chamber.

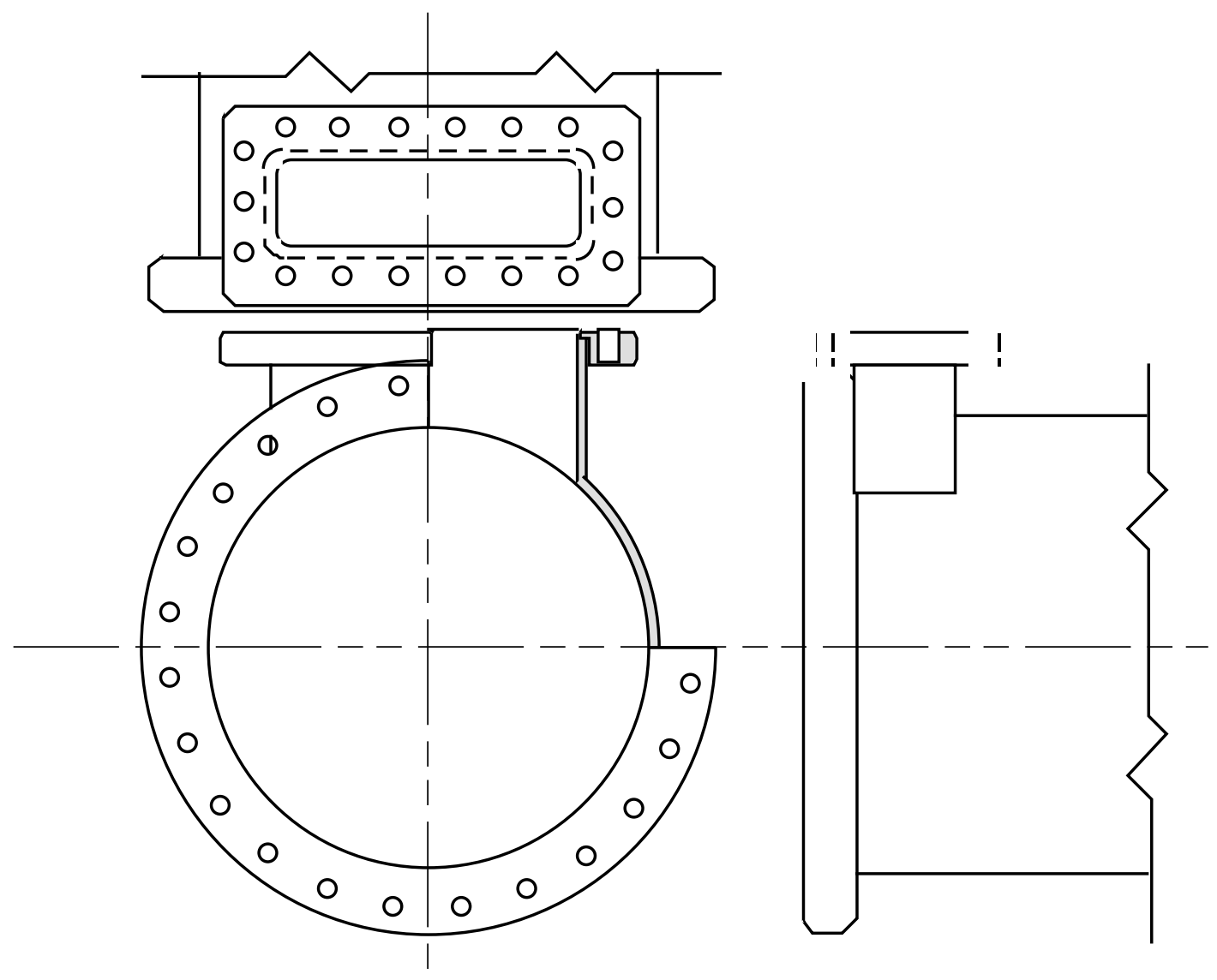

Figure 5. Design of the rectangular detector port modification to the time -of-flight mass spectrometer vacuum housing. 
The internal components of the new jet-REMPI laser ionization source were purchased from R. M. Jordan. The source consists of a repeller plate, extraction grid, acceleration grid, Einsel lenses, and steering plates. The laser-ionization source was installed in the source vacuum chamber along with a pair of quartz windows for laser access.

Pumping for the instrument was originally provided by two, $250 \mathrm{~L} / \mathrm{sec}$ Varian turbomolecular pumps. One pump was located on the lower flange of the source chamber shown in Figure 4, while the second pump was located on the flight tube. The latter was a standard V250 type pump, while the former was a V-250SF split-flow type. The split-flow pump incorporates an intermediate pumping port that was used to provide backing for the V-250 pump. The two turbomolecular pumps are backed by a $450 \mathrm{~L} / \mathrm{min}$ Varian mechanical pump. The allmetal vacuum housing for the time-of-flight mass spectrometer system routinely achieves pressures in the mid $10^{-8}$ Torr range with no sample present. Under routine operation, the pressure in the source region rises to $5 \times 10^{-5}$ Torr, and the pressure in the flight tube rises to $2 \mathrm{x}$ $10^{-7}$ Torr. The pumping system was subsequently upgraded in conjunction with the new source chamber (see Section 3.1.8).

\subsubsection{Pulsed Valve}

The pulsed valve selected for the prototype laboratory instrument is an unmodified, commercial, General Valve Series 9 type. This valve was ordered with the high-temperature coil option, in anticipation of heating above $150^{\circ} \mathrm{C}$.

The pulsed valve was fitted with a copper heating block to allow for thermal control of the valve temperature. Two 50-watt cartridge heaters were inserted into the copper block to provide heating, while a type-K thermocouple also inserted into the block is used to measure the temperature. A standard proportional temperature controller is used to maintain the valve at desired temperature. An vacuum insert was fabricated to position the pulsed valve close to the ion extraction optics. A cross sectional view of the insert, shown in Figure 6, includes an outline of the heater block and the pulsed valve.

A movable mounting system was fabricated using a bellows-sealed vacuum translator to position the valve. This system is shown schematically in Figure 7, along with the ion extraction optics. The main component of the translator is a commercial, MDC unit capable of providing $7 \mathrm{~cm}$ of linear travel. As shown in Figure 7, the bellows-sealed translator is mounted on the large top flange of the ion source vacuum chamber. In the figure, the insert is at its lowest position within the source chamber, and hence the molecular jet is closest to the laser ionization region. Adjustment of the position of the jet relative to the laser beam allows optimization of the 
signal that results from ionizing molecules that have achieved the maximum degree of cooling. In our later design, we eliminated the movable feature and fixed the separation at the optimum position. The fixed location pulsed valve configuration is described in Section 3.1.7.

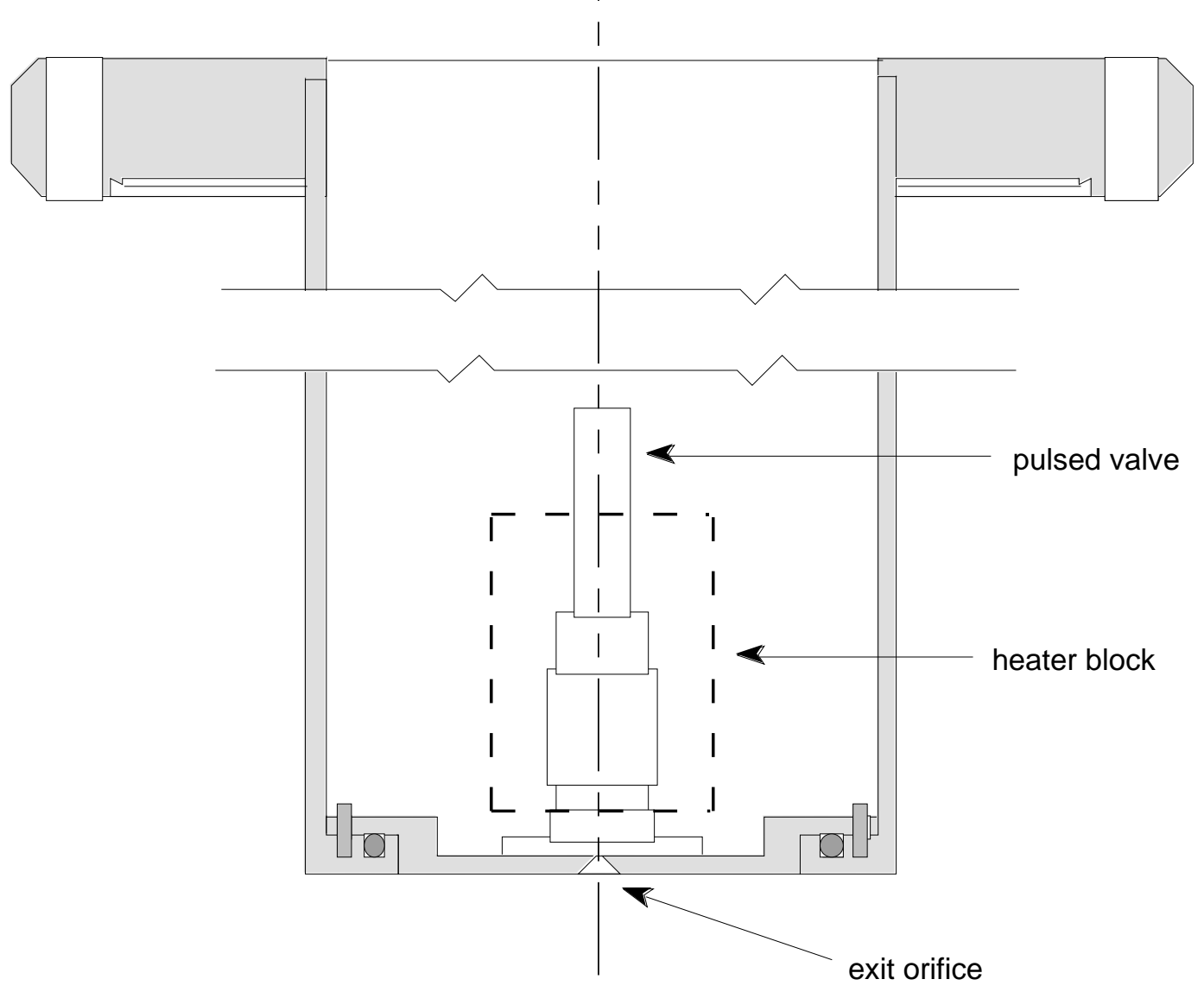

Figure 6. Design of the pulsed valve positioning device

Many pulsed valve designs have been developed based on simple electromechanical solenoid mechanisms, piezoelectric actuators, magnetic repulsion devices, and modified fuel injectors. Pulsed durations range from a few microseconds to hundreds of microseconds, while repetition rates reach $80 \mathrm{~Hz}$ or higher, depending on the pulse duration and valve design. A critical parameter for the pulsed valve in a dioxin CEM is the temperature at which it can operate. Because of the low volatility of the heavier dioxin congeners, the inlet sampling lines and pulsed valve must be continuously heated above $250^{\circ} \mathrm{C}$. Although many of the earlier valve designs could not be heated much above $100^{\circ} \mathrm{C}$ due to the temperature limits of the sealing materials, designs ${ }^{13,14}$ now exist for pulsed valves that can operate routinely at temperatures 
above $400^{\circ} \mathrm{C}$. These designs will form the basis for our design of a high-temperature pulsed valve.

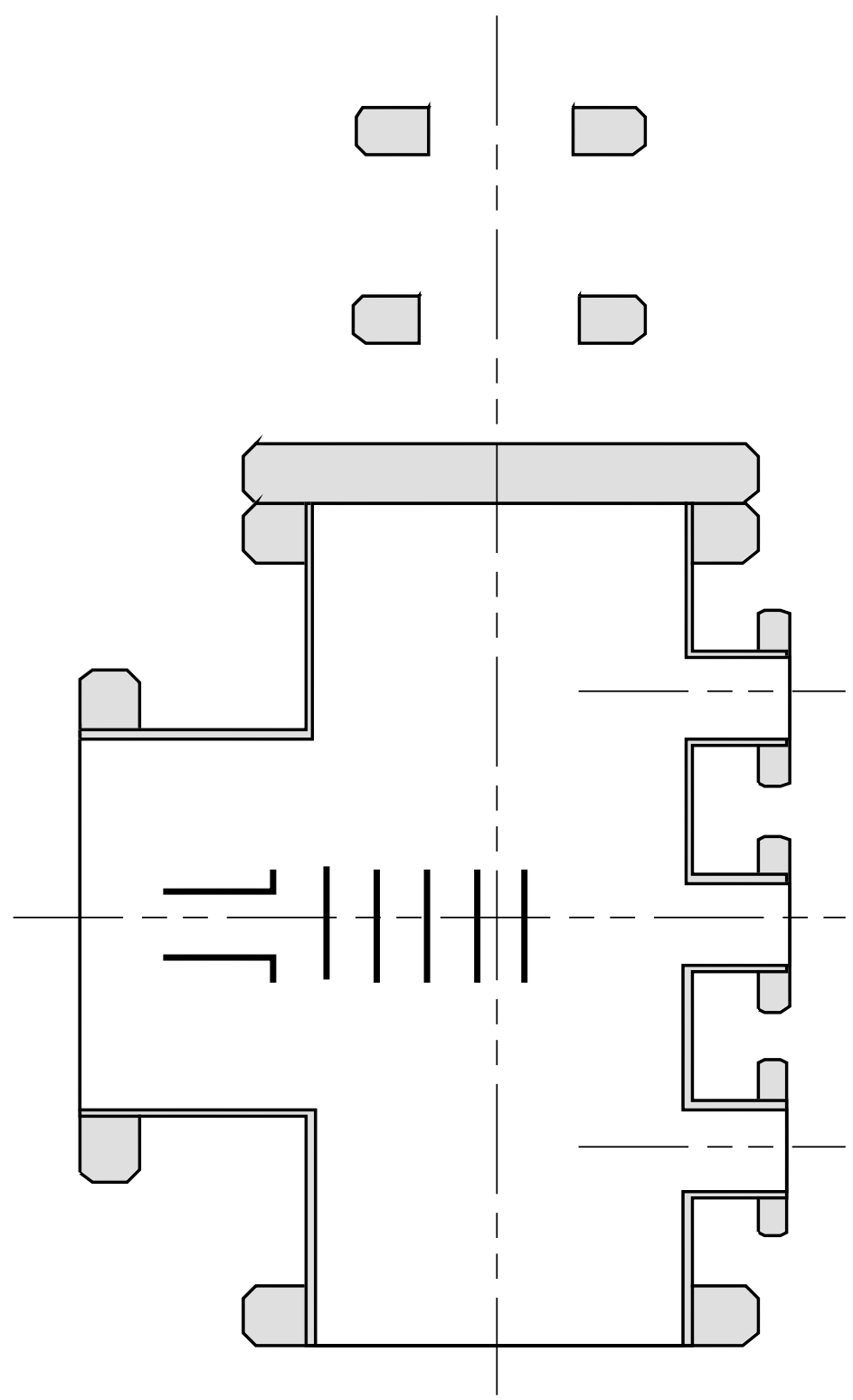

Figure 7. Schematic of the valve positioning system installed on the ion source vacuum housing. The ion extraction optics are shown in the center of the lower chamber.

\subsubsection{Tunable uv Laser}

The laser system required for REMPI of dioxins must be capable of providing tunable, narrow band radiation in the range between 220 and $350 \mathrm{~nm}$. At present, the most reliable 
method of generating this laser radiation is through the use of an optical parametric oscillator (OPO) pumped by a Nd:YAG laser. Commercial Nd:YAG lasers are very well-developed, stable, and reliable solid-state systems capable of delivering millions of pulses with minimal maintenance. Modern OPOs are also relatively reliable and stable systems. They provide many advantages over the tunable dye lasers they have replaced. OPOs are all solid-state devices that are easily controlled by a computer. They use no consumables and can provide wavelength scanning over a broad visible wavelength range, typically between 440 and $750 \mathrm{~nm}$. Frequency doubling in a nonlinear, solid-state crystal, provides the tunable uv radiation. Once they are adjusted, OPOs require minimal maintenance and only occasional recalibration.

At the beginning of this project, SRI had available a Continuum Powerlite $9010 \mathrm{Nd:YAG}$ pump laser system. Using capital equipment funds, SRI purchased a Continuum Sunlite OPO. The Powerlite was returned to the factory where it was upgraded to drive the new OPO. The upgrade included replacement of the flashlamps and laser rods, installation of improved electronics, and realignment. In addition, an intermediate optics housing located between the $\mathrm{Nd}$ :YAG and the OPO was purchased for installation during the upgrade. This unit allows either the fundamental or one of the harmonics to be diverted out of the laser and used independently of the OPO.

Our Continuum Nd:YAG pump laser and new OPO were delivered at the end of February 1999, and the actual installation was performed at the beginning of March. The factory refurbished pump laser and the OPO laser specifications are given in Table 2. All operating parameters meet or exceed our requirements for using this system in the jet-REMPI experiments.

Measured output energies from the Sunlite OPO over the important wavelength range from $680 \mathrm{~nm}$ to $460 \mathrm{~nm}$ averaged $45 \mathrm{~mJ}$ with the amplifier being pumped with $180 \mathrm{~mJ}$ of $355 \mathrm{~nm}$ radiation.

Table 2

PERFORMANCE SPECIFICATIONS OF THE CONTINUUM LASER SYSTEM

\begin{tabular}{lcc}
\multicolumn{1}{c}{ Parameter } & Specification & Actual \\
\cline { 4 - 4 } $1064 \mathrm{~nm}$ pulse energy (unseeded) & $2000 \mathrm{~mJ}$ & $>2000 \mathrm{~mJ}$ \\
$355 \mathrm{~nm}$ third harmonic energy & $400 \mathrm{~mJ}$ & $420 \mathrm{~mJ}$ \\
$1064 \mathrm{~nm}$ pulse width & $5-9 \mathrm{~ns}$ & $8 \mathrm{~ns}$ \\
$355 \mathrm{~nm}$ pulse width & $3-7 \mathrm{~ns}$ & $6.2 \mathrm{~ns}$ \\
$1064 \mathrm{~nm}$ shot-to-shot stability & $\pm 2.5 \%$ & $\leq 1 \%$ \\
$355 \mathrm{~nm}$ shot-to-shot stability & $\pm 4.0 \%$ & $\pm 1.5 \%$
\end{tabular}


As noted, tunable radiation in the ultraviolet region is required for REMPI of dioxins and other aromatic hydrocarbon species. Generation of this uv radiation from the visible OPO output was achieved by using an Inrad Autotracker II doubler. The Autotracker uses a feedback control system and stepper motors to continuously adjust the alignment of its optical components during wavelength scanning. The Autotracker II provides barely minimal performance when used with the Continuum OPO. The high scanning rate of the OPO, which is necessary for fast time response of the instrument, causes the Autotracker to become unstable and oscillate rather than smoothly track the changing wavelength. The Autotracker was ultimately replaced with a Continuum FX-1 doubler. The FX-1, described in Section 3.1.5, is designed to operate with the $\mathrm{OPO}$ and provides significantly more stable operation.

\subsubsection{Mass Spectrometer}

A primary consideration in the choice of a mass spectrometric analysis technique is the method of ion formation. For a REMPI-based CEM, the mass spectrometric method of choice is time-of-flight (TOF). TOF is an inherently pulsed technique that matches well with the pulsed laser ionization, and it offers the advantage of generating a complete mass spectrum from each laser pulse.

The mass spectrometric requirements of a dioxin CEM instrument are modest: an upper mass range of 400, with unit mass resolution. Typical TOF mass analyzers can meet these requirements using a simple, linear flight path when combined with a short duration, pulsed laser, ionization source. The low signal levels that may result from the use of a very narrow ion formation pulse can be offset for by signal averaging over multiple laser pulses. To optimize this mode of operation, a high repetition rate ionization laser would be desirable. Achieving high mass resolution with a TOF instrument is straightforward, using a pulsed laser for ionization, because of the narrow spatial, temporal, and energy spread of the nascent ions.

An increasingly common TOF approach is the use of an ion mirror, or reflectron-type TOF. This device has many desirable properties and is the approach taken in our instrument. Resolving powers $(\mathrm{m} / \Delta \mathrm{m})$ of more than 1000 can be readily obtained using a reflectron with a pulsed laser ionization source. Although mass resolving powers in excess of 1000 are not necessary to separate adjacent mass peaks even at the highest mass-to-charge ratios expected in a dioxin CEM, high resolution gives rise to signals with much narrower, and hence much higher, peaks. The increased peak amplitude leads directly to an increased signal-to-noise ratio.

A significant improvement in resolution and signal level can also be obtained through the careful design of the ion extraction optics. As a result of the pulsed laser ionization scheme, ion 
formation is constrained to occur over a very limited spatial region. Normally, a wellconstrained ion formation region can be readily coupled with high efficiency to a TOF mass analyzer using simple ion extraction optics. In our instrument, however, an additional spatial constraint is imposed by the use of a pulsed gas inlet. Pulsed valves can produce a supersonic molecular beam as the gas expands and cools adiabatically. An optimum location downstream from the orifice exists where the internal molecular temperature will have reached a minimum while the local gas density remains high. Ionization at this location will yield the optimum optical spectroscopic selectivity with maximum ion signal level. Because this optimum ionization region will typically be only a few centimeters from the exit orifice, the ion optics must be carefully designed to include the influence of the pulsed valve on the extraction fields.

We therefore undertook a detailed simulation of the ion extraction optics for the time-offlight mass spectrometer. A critical design parameter affecting the detection sensitivity of the jet-REMPI scheme is the location of the exit of the pulsed nozzle relative to the ionization laser. This distance influences the signal in two ways: (1) the large, grounded, valve body can distort the ion extraction and focusing fields, and (2) the gas density and degree of internal cooling of the supersonic expansion varies with position. Using the standard simulation program, SIMION, we modeled all aspects of the ion extraction optics to determine an optimum initial configuration of the key components. The results of these simulations were incorporated into the design of the pulsed valve mount and ion extraction optics.

\subsubsection{New Optical Frequency Doubler}

In the early part of the Phase 2 effort, it became necessary to move the location of the jetREMPI instrument. Because moving the instrument involves repositioning of the laser system which necessitates optical realignment, we took the opportunity to install a commercial laser table in place of the homemade table that had been used previously. The new table is a standard Newport 4' x 8' honeycombed table with predrilled holes for optical mounts. Because moving and remounting the laser system requires realignment by the manufacturer. This realignment was done in conjunction with the installation of a Continuum FX-1 doubler system. The FX-1 replaced the Inrad Autotracker system that had been used to convert the tunable visible output of the OPO into the required uv. Because the Inrad unit was not integrated into the OPO system, its ability to track the wavelength tuning was poor, resulting in output instabilities. The FX-1 is totally integrated into the OPO system, eliminating these problems and significantly improving the output stability of the laser. The FX-1 was purchased as part of SRI's EPA project on urban air toxics, which shared the laser system with this effort. 


\subsubsection{New Control Program}

A major thrust during the second phase was the development of new control and data acquisition software. Although the existing software, written in Visual Basic 6.0, was sufficient for most tasks, our planned improvements would have required considerable changes in the code. For a variety of reasons, we concluded that National Instruments LabView offered a better development platform. LabView is generally considered to be an industry standard for instrument control and real-time data acquisition. The current version of our control program was developed using LabView 6.0.

The baseline program functioned correctly, and was able to control the laser wavelength, acquire time-of-flight data from the digitizer, and display and store the resulting mass spectra. Additional features were added to the software, specifically improved calibration methods. The general approach to automating the calibration is to provide the user with a list of possible calibrant species for which the software has predetermined wavelengths and expected masses. Selection of the calibrant would then automatically sequence through the wavelengths, recording and displaying the intensities of the expected masses. Repeating this data collection as a function of calibrant concentration would yield a calibration curve.

Although the new control program functioned as expected, a potentially serious problem was found associated with the averaging function. It was found that 100 laser shots at $10 \mathrm{~Hz}$ that should have required exactly 10 seconds, actually required 20 seconds to complete. The timing problem was determined to be due to an automatic power down feature of the Signatec DA500A digitizer. This feature, designed to prevent the board from overheating during prolonged use, had not been properly disabled during the 100 shot burst. Hence the digitizer periodically reverted to a low power mode which introduced an additional $100 \mathrm{msec}$ delay when the next scan was triggered. Since the LabView driver that we have used to interface with the Signatec card did not provide control over this feature, we implemented the necessary changes to the driver and overcame this limitation. Data collection now occurs with no time overhead so that 100 laser shots at $10 \mathrm{~Hz}$ requires just 10 seconds.

\subsubsection{Internal Temperature}

The effective internal temperature of sample molecules in the supersonic jet is important for determining both sensitivity and selectivity of the jet-REMPI method. To determine the optimum operating conditions for obtaining the lowest temperature in combination with the maximum signal level, we performed two types of experiments. First, the sensitivity and temperature distribution in the pulsed supersonic beam was measured as a function of the valve opening time. The opening time directly affects the collisional behavior in the supersonic beam, and to a lesser extent, the density distribution. The latter is expected to increase as the opening time is increased, achieving a maximum value for opening times greater than the time required 
for the pulsed valve to fully open. The second type of measurement determined the effect on sensitivity and rotational temperature of the distance between the gated valve and the laser beam. This distance is important for allowing the collisional cooling to extract as much random thermal energy from the molecular beam as possible. Benzene was used as a thermometric species owing to its well-established REMPI spectroscopy. In particular, the ${ }^{1} \mathrm{~B}_{2 \mathrm{u}} \leftarrow{ }^{1} \mathrm{~A}_{1 \mathrm{~g}} 6{ }_{0}{ }_{0}$ band near $259 \mathrm{~nm}$ was studied. Band profiles were calculated as described below and fitted to the measured spectra, allowing a determination of the temperature to an accuracy of approximately $1^{\circ} \mathrm{K}$.

Band profiles require calculation of the individual relative line intensities, $\mathrm{I}_{\mathrm{KJ}}$ which are given by

$$
\mathrm{I}_{\mathrm{KJ}}=\mathrm{C} \vee \mathrm{A}_{\mathrm{KJ}} \mathrm{g}_{\mathrm{KJ}} \mathrm{e}^{-\mathrm{F}(\mathrm{K}, \mathrm{J}) \mathrm{kT}}
$$

where $\mathrm{C}$ is proportional to the Franck-Condon Factor (constant for a single vibration level), $v$ is the photon frequency, $A_{\mathrm{KJ}}$ is the line strength (given by the Hönl-London formulae for a perpendicular transition in a symmetric top), $\mathrm{g}_{\mathrm{KJ}}$ are the statistical weights of the lower state (for molecule with $\mathrm{D}_{6}$ rotational symmetry), $\mathrm{F}(\mathrm{K}, \mathrm{J})$ is the energy of the lower level, $\mathrm{J}$ is the total angular momentum and $\mathrm{K}$ is the projection of $\mathrm{J}$ onto the figure axis of the symmetric top, both for the lower state. A complete band consists of $\mathrm{P}, \mathrm{Q}$, and $\mathrm{R}$ subbands corresponding to $\Delta \mathrm{J}=0, \pm 1$, with subband origins distributed along Fortrat parabolas corresponding to $\Delta \mathrm{K}= \pm 1$. Accurate band profiles require that Coriolis energies be included for the upper state.

We chose benzene as the test molecule because its optical absorption spectrum is well characterized. We made measurements of a single band contour for benzene, shown in Figure 8. This spectrum was taken at a distance of $2.3 \mathrm{~cm}$ from the nozzle following a delay from the valve opening that provided the best ion intensity and cooling for this distance. The band shown is part of the lowest singlet-singlet transition $\left(S_{1} \leftarrow S_{0}\right)$ of benzene near $259 \mathrm{~nm}$. This specific transition has the spectroscopic identification ${ }^{1} \mathrm{~B}_{2 \mathrm{u}} \leftarrow{ }^{1} \mathrm{~A}_{1 \mathrm{~g}} 6^{1}{ }_{0}(1= \pm 1)$. The band shown corresponds to many overlapping rotational lines. From the overall contour of these overlapping lines, we can determine an effective rotational temperature of the benzene molecules.

To determine the temperature, we wrote a computer program to fit the simulated the form of the band contour as a function of temperature. This program used the most recent values for the spectroscopic parameters for the $\operatorname{transition}^{13}$ and the appropriate statistical weights ${ }^{14}$. The result of this fitting program gives the best estimate of the rotational temperature of the benzene molecules, which is $18 \pm 1 \mathrm{~K}$ for this measurement. The high quality of the calculated fit is shown in Figure 8. This rotational temperature is very low, and indicates that our gas expansion is near optimum in terms of its cooling characteristics. 


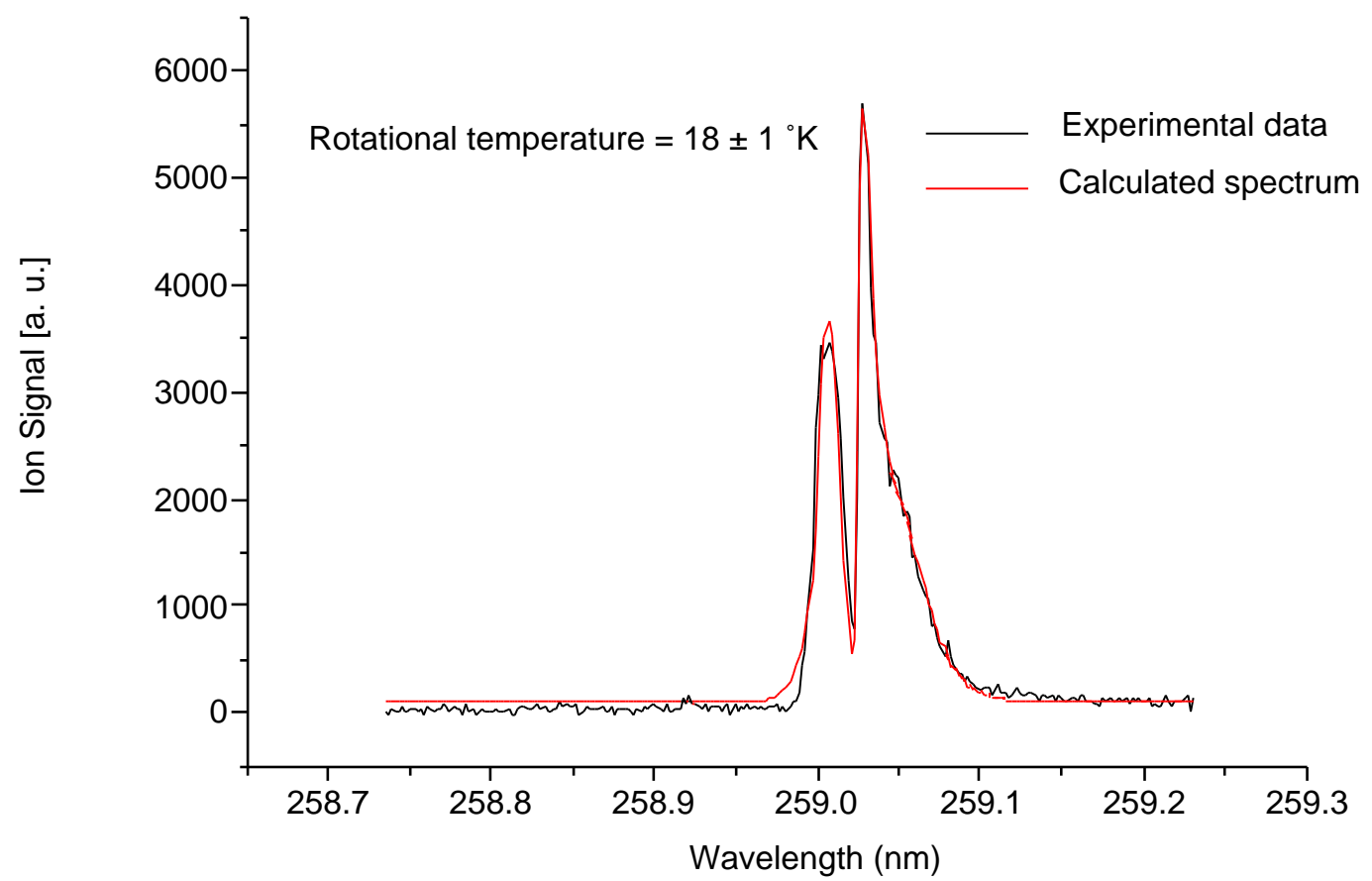

Figure 8. Comparison of the measured and calculated band profiles for a valve-to-laser separation of $2.3 \mathrm{~cm}$ for a $\mathrm{S}_{1} \leftarrow \mathrm{S}_{0}$ transition in benzene.

To complete this initial series of measurements, we determined the REMPI signal for the same band in benzene as a function of the valve-to-laser distance. These measurements provided two important pieces of information; the local gas density as a function of distance, and the degree of internal cooling as a function of distance. Figure 9 shows a plot of the local gas density, as determined by the integrated REMPI signal, as a function of the inverse square of the valve-to-laser separation. In this representation, the expected quadratic falloff in intensity with distance should result in a straight line. A second order polynomial produces an excellent least squares fit to our data with a correlation coefficient of 0.996. The minimum distance for which we could measure the REMPI signal was approximately $2.3 \mathrm{~cm}$, while the maximum was approximately $7.4 \mathrm{~cm}$.

While this quadratic dependence of intensity with distance is to be expected, the dependence of the internal cooling with distance was not anticipated. Using our temperature fitting program, we determined that there was essentially no change in the rotational temperature of the benzene molecules as a function of the valve-to-laser distance. This surprising result implies that molecular cooling is complete within a very short distance from the exit of the jet nozzle. Based on this observation, the maximum REMPI signal intensity is only a function of the local gas density, which is easily estimated from the quadratic behavior exhibited in Figure 9. 


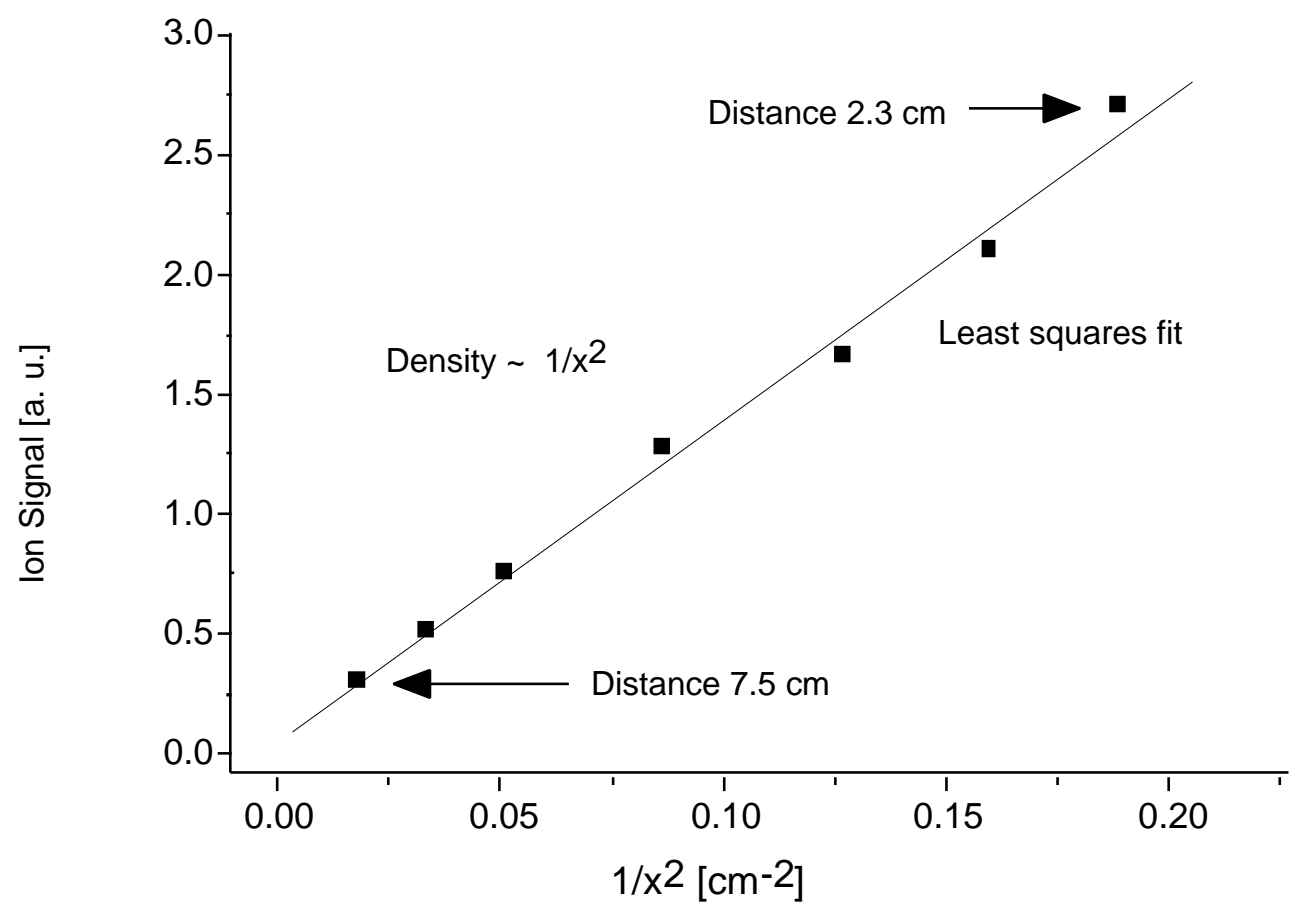

Figure 9. Variation of the jet-REMPI signal for benzene measured at 78 amu and a wavelength of $259.1 \mathrm{~nm}$ as a function of the laser-valve separation.

At the smallest valve-to-laser separation of $2.3 \mathrm{~cm}$, the measured and calculated band profiles were shown in Figure 8. Figure 10 shows the corresponding results for the largest valveto-laser separation of $7.5 \mathrm{~cm}$. The best fit rotational temperature for this data set was $18.5 \pm 1{ }^{\circ} \mathrm{K}$.

The relative lack of a strong temperature dependence on the valve-to-laser separation is not unexpected. At a separation of $2.3 \mathrm{~cm}$, the benzene molecules are most likely already in a collision-free regime, hence, no further reduction in temperature will occur. While the valve-tolaser separation does not influence the internal temperature of the beam, the $1 / \mathrm{x}^{2}$ drop in local density measured in Figure 9 confirms the importance of fixing the valve-to-laser separation at the smallest value possible consistent with the mechanical constraints of the ion extraction optics and the gas pumping.

The other key variable in optimizing the REMPI signal is the delay between the valve pulse and the ionization laser pulse. To investigate the dependence of the benzene REMPI signal on this parameter, we made a series of band profile measurements while varying the delay time. The results are shown in Figure 11. The solid line in Figure 11 shows the variation in the signal strength of the 78 amu benzene parent ions measured with a time delay of between 300 and 1100 $\mu \mathrm{s}$. As expected, the ion signal initially rises as the delay time increase, reflecting the increase in 


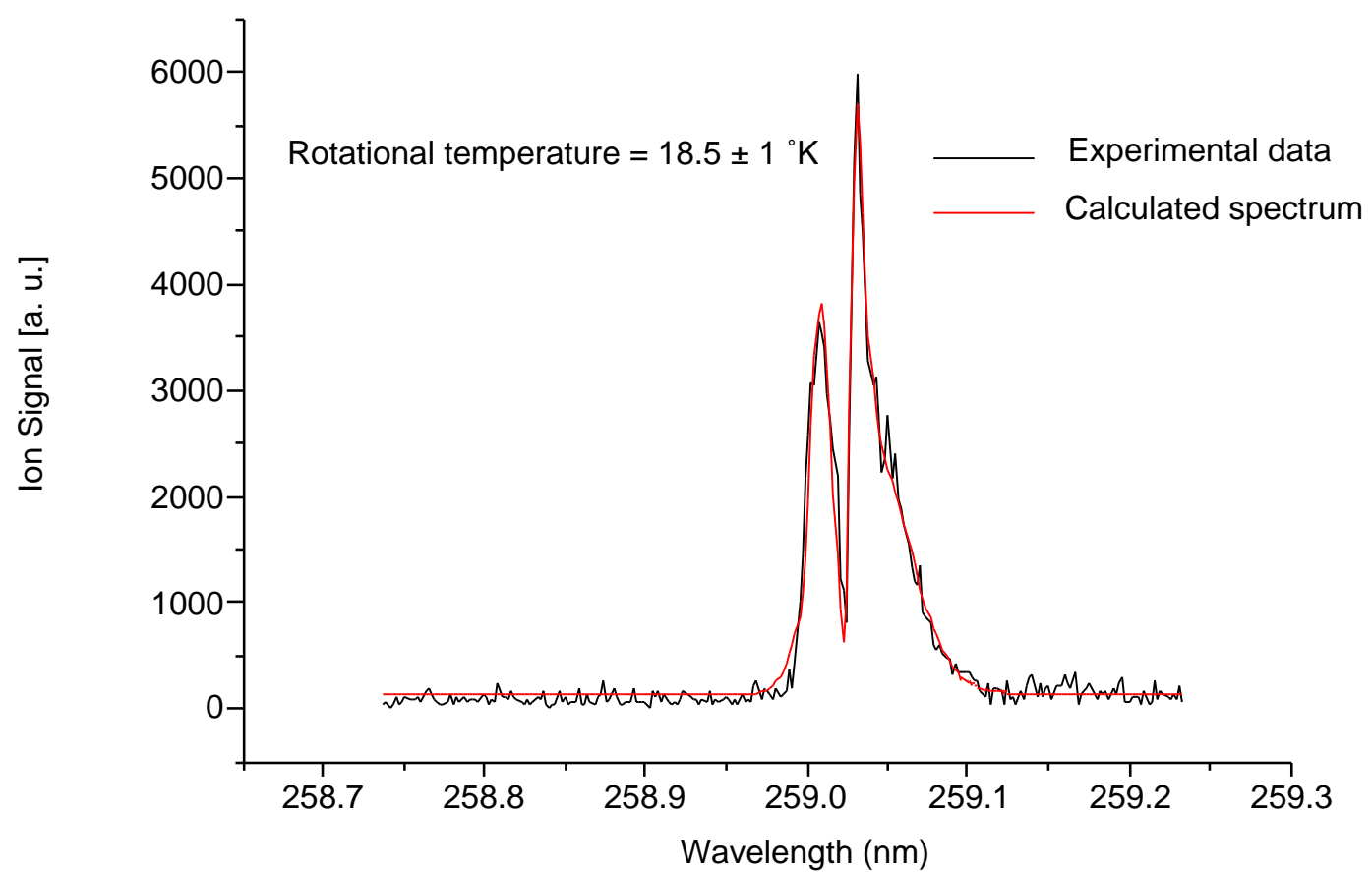

Figure 10. Comparison of the measured and calculated band profiles for a valve-to-laser separation of $7.5 \mathrm{~cm}$ for a $\mathrm{S}_{1} \leftarrow \mathrm{S}_{0}$ transition in benzene.

gas density as the molecular beam forms and flows past the ionization region. As the delay time become longer than approximately $450 \mu \mathrm{s}$, the signal level begins to decrease as the bulk of the gas pulse has passed the laser beam.

Also shown in Figure 11 are the fitted rotational temperatures measured at a number of time delays. Using only a simple vertical scaling of the two sets of data, we find that the rotational temperature exactly follows the gas density variation. This indicates that the peak density corresponds to the coolest neutral molecules. Presumably, this correspondence arises because the cooling mechanism depends on intermolecular collisions, which will be most probable when the local gas density is at a maximum. That these two dependencies occur at a common time delay greatly simplifies the optimization of the signal with respect to this parameter.

\subsubsection{New Ion Source Chamber}

The original jet-REMPI laser ionization source chamber was designed to maximize flexibility and versatility. It incorporated a precision translation stage to allow adjustment of the distance between the exit of the pulsed nozzle and the laser ionization zone. While this capability proved to be very useful in optimizing the performance of the system, it is now possible to fix this distance at an optimum valve and hence we can eliminate the translation stage and simplify the instrument. Elimination of the translation stage involved fabrication of a new 


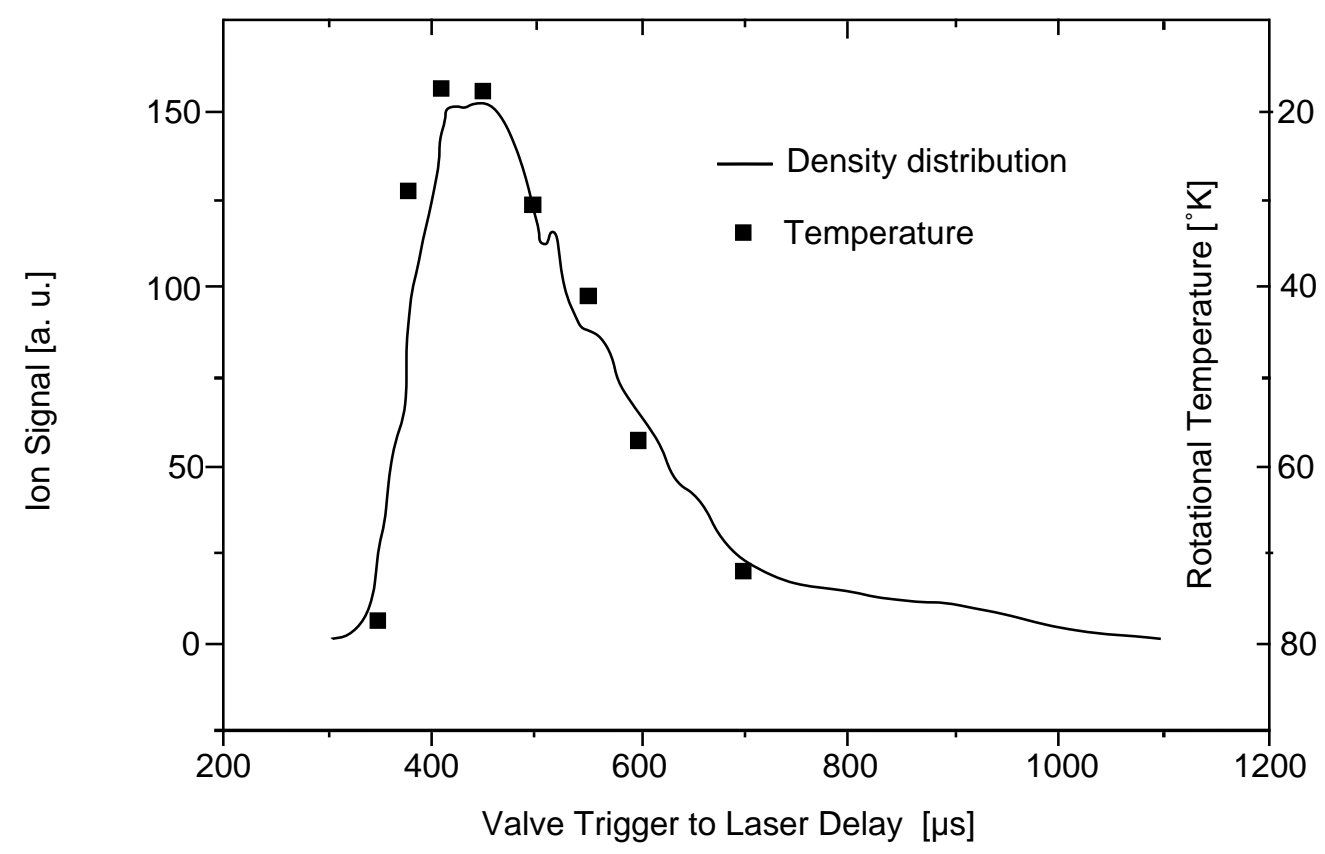

Figure 11. Variation of the jet-REMPI density distribution (signal level) for benzene measured at 78 amu and a wavelength of $259.1 \mathrm{~nm}$ as a function of the delay between the valve trigger and the laser pulse. The corresponding rotational temperature as determined by fitting the band profiles is also shown.

source chamber. A cross sectional drawings of the new chamber is shown in Figure 12. Note that a new valve assembly, described in detail below, is positioned at a fixed distance from the laser beam. The outer body of the vacuum chamber was modified to move the valve as close as possible to the ion extraction optics without inducing an appreciable distortion in the electric field potentials required for extraction and acceleration of the ions. This design also provides maximum pumping in the pulsed gas jet region, which is important in maintaining a low pressure in the ion source region.

The new chamber also allows us to overcome an operational problem associated with the use of a single pulsed valve. With a single pulsed valve, it is not possible to perform any routine maintenance without venting the entire apparatus to atmosphere. Replacement of the sealing poppet is a typical example of an operation requiring considerably longer to complete because the vacuum system must be vented and re-evacuated. In addition, we have found that it is difficult to remove residual traces of many of the sample compounds that we have been studying.

These compounds tend to have low vapor pressures and adhere to surfaces within the pulsed valve. Even with prolonged heating, we are able to detect minute traces of samples that have nominally been removed from the inlet. This is of course a result of the exceptional sensitivity of the instrument. 


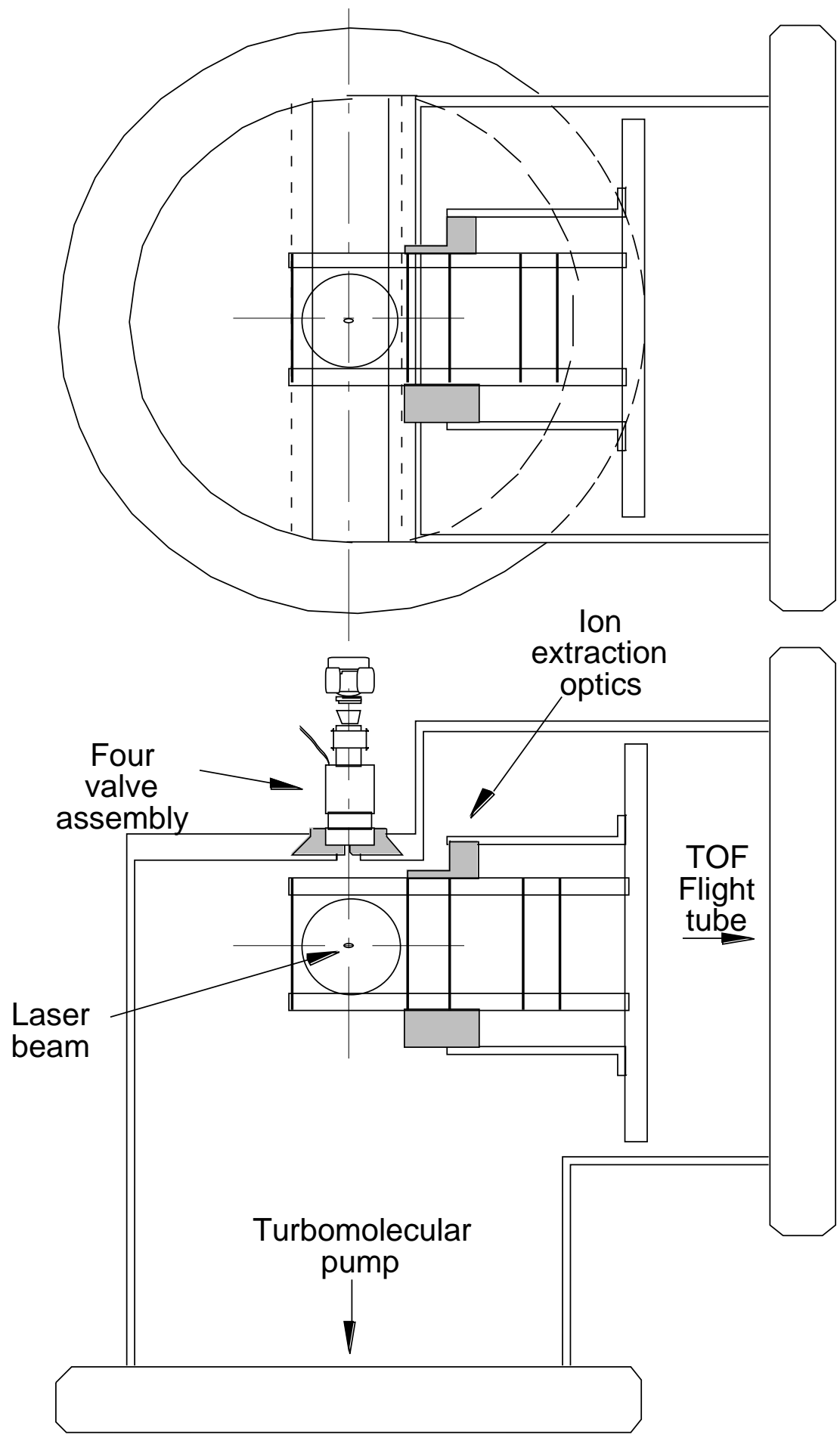

Figure 12. Schematic of the new ion source vacuum chamber including the sliding four-valve assembly.

\subsubsection{4-Valve Inlet Assembly}

To circumvent the above described shortcomings of the original pulsed valve design, we designed a new valve assembly that incorporates four pulsed valves on a sliding mount. The 
new source chamber was fabricated to accept this assembly in place of the single pulsed valve. The sliding valve mount, shown in Figure 13, was designed to allow any of the four valves to be positioned over the ionization region without loss of vacuum. This capability permited us to (1) remove any of the inactive valves for servicing without vacuum loss, (2) rapidly switch from a valve with residual sample contamination to a clean valve, and (3) quickly investigate the effect of varying the nozzle diameter on sensitivity.

Figure 14 shows the configuration of the four valve assembly. This unit was custom fabricated for SRI by General Valve based on our design. It incorporates four standard series 9 valves in a sliding base block. Figure 14 shows a cross section of the base and one of the valves.

Heating of the pulsed valves is very important, especially when dealing with the more highly chlorinated dioxins. Experience has shown that temperatures of at least $200 \mathrm{deg} \mathrm{C}$ are required to avoid unacceptable sample loss due to condensation. To achieve this temperature, we have designed and fabricated a large copper heater block that surrounds all four valves. The block extends from the sliding base to a point just below the Swagelok ${ }^{\circledR}$ fitting on the top of the valves. This block has four, 0.25 " diameter channels drilled through its length to accommodate four, 200-Watt cartridge heaters. In addition, the sliding base has four, 0.125 " diameter channels drilled through its length to accommodate four, 50-Watt cartridge heaters. These two sets of heaters will be independently controlled to maintain the valves at an elevated temperature. This separation in control zones was necessary because of the relatively large thermal mass of the latter components as compared with the former components.

\subsubsection{New Jet-REMPI TOFMS}

While fabricating a new jet-REMPI system for delivery to the US EPA, we simultaneously assembled a duplicate TOFMS for use on this project, as well as our EPA HAPs effort. The majority of the components were purchased using SRI internal funds. We completed assembly and testing of a new jet-REMPI system during the second phase of our project. The internal ion optics of this new system were initially the same as our functioning system, however, provisions have been made to implement the slit nozzle in the near future. Pumping for the new instrument includes a $550 \mathrm{l} / \mathrm{sec}$ turbomolecular pump for the source region, and a smaller, $250 \mathrm{l} / \mathrm{sec}$ turbomolecular pump for the flight tube. This system, assembled using support from SRI, will be used to measure the spectroscopic signatures and detection limits of target compounds. This instrument makes use of the new ion source vacuum chamber and fourvalve assembly described above. These modifications, along with the larger vacuum pump, will simplify testing of the slit nozzle and rapid switching between varying sample streams. 


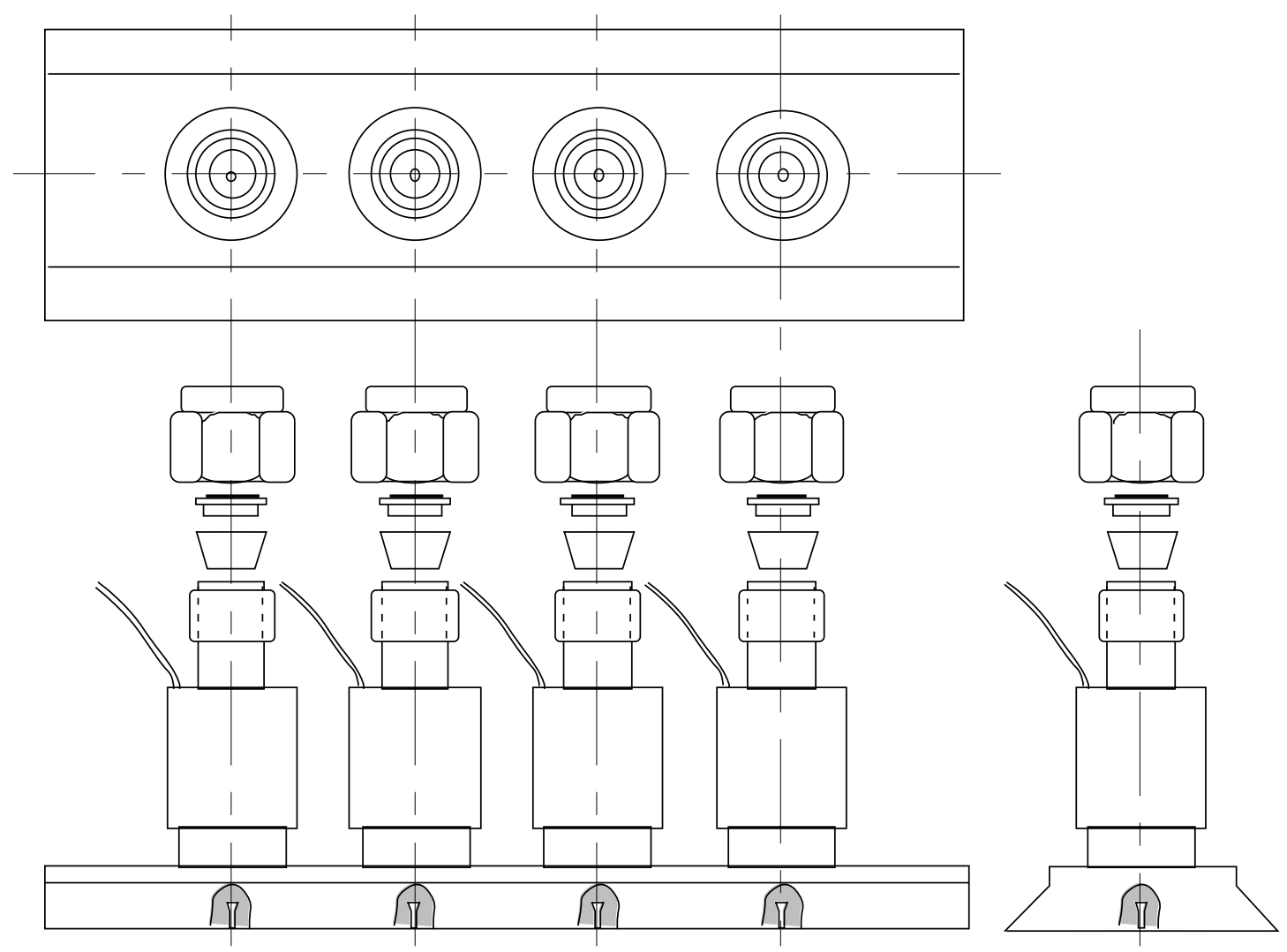

Figure 13. Configuration of the custom-fabricated, four-valve assembly.

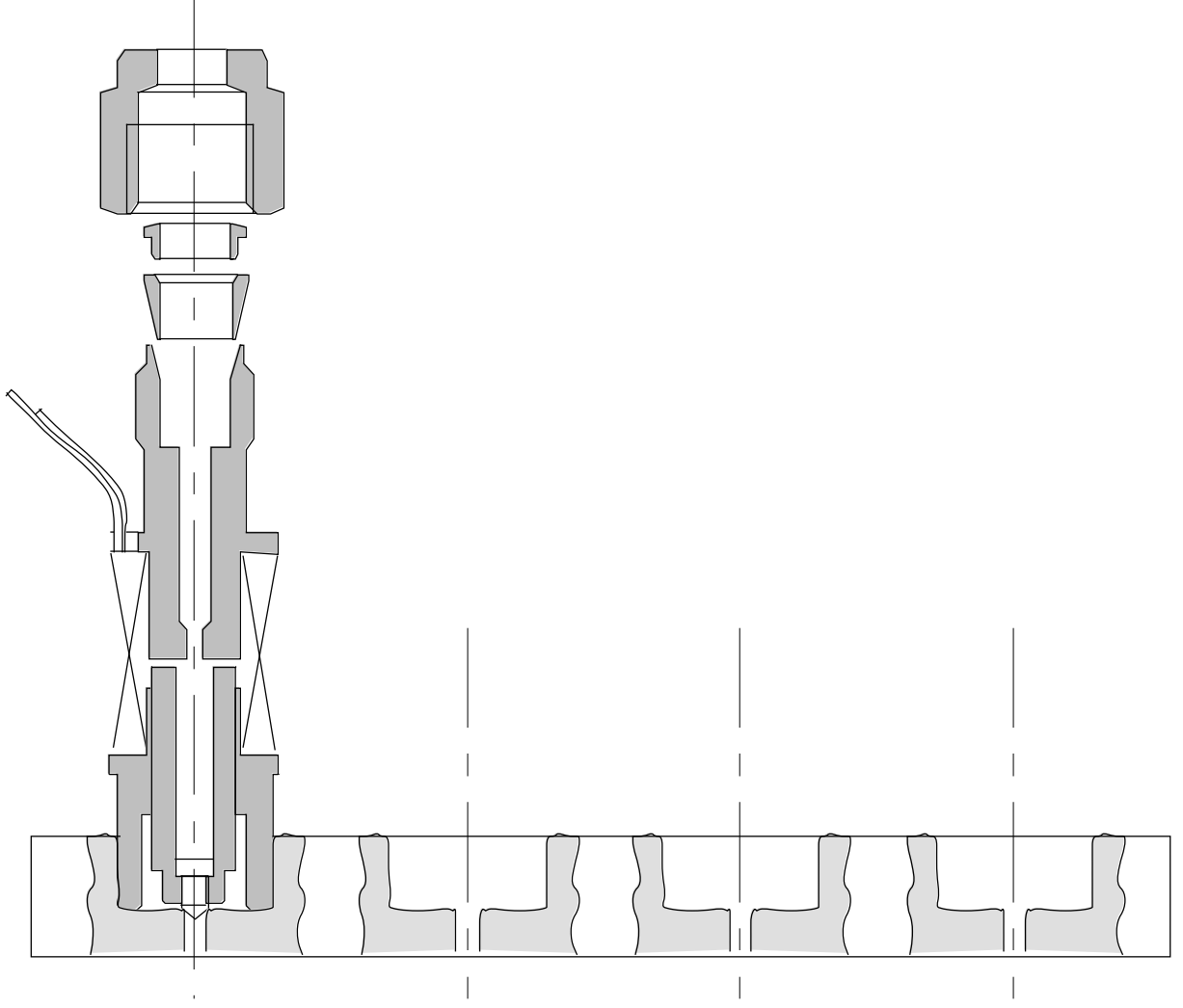

Figure 14. Cross sectional view of one valve and the base plate of the four-valve assembly. 
The new system can be used either with the same laser system as the existing jet-REMPI instrument, or it can be easily moved to another laboratory where a similar laser system is available for occasional use. Access to the second laser system provides an opportunity to simultaneously perform two different experiments using the two mass spectrometer systems. A photograph of the new TOFMS and existing laser system is shown in Figure 15.

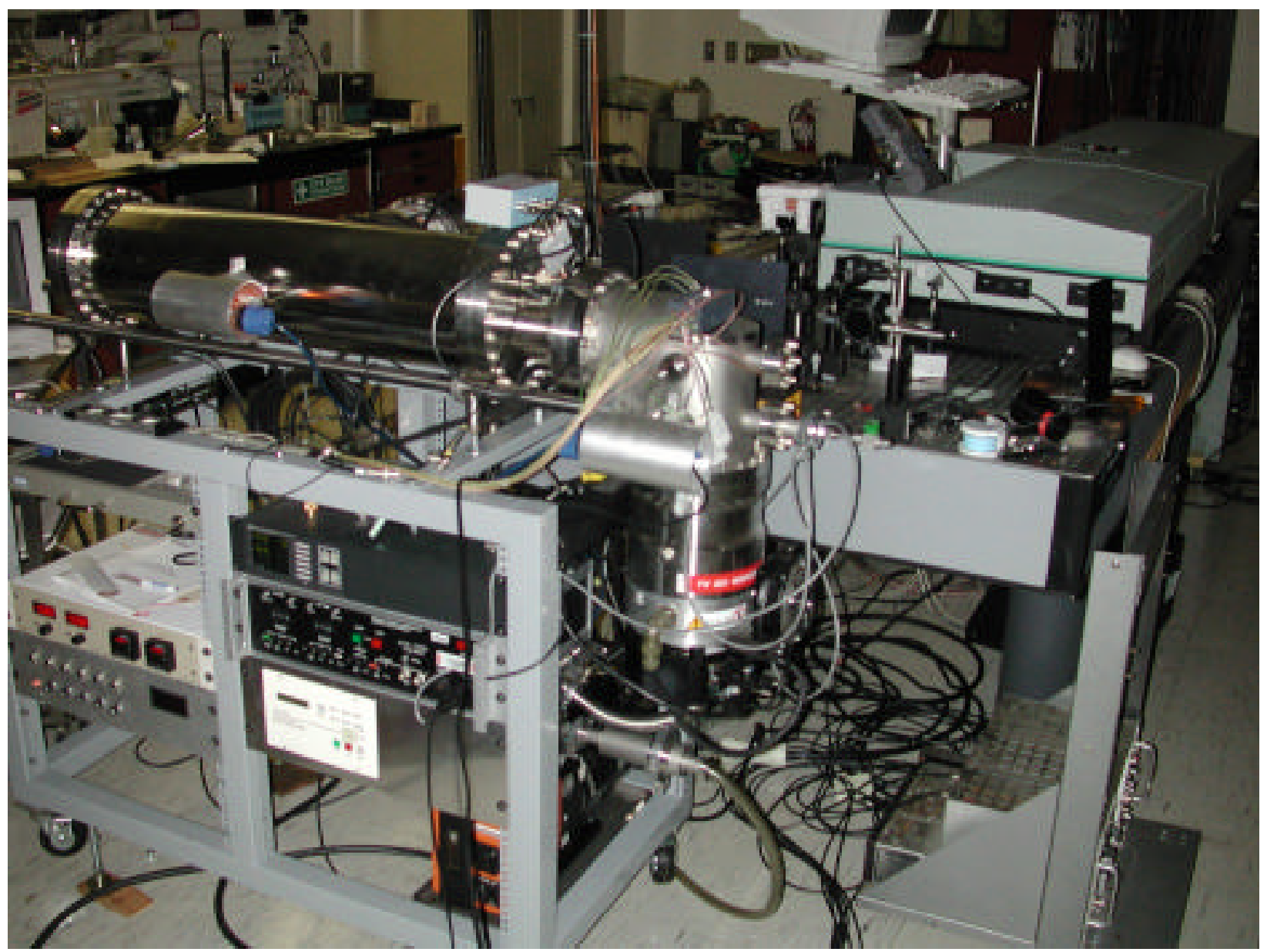

Figure 15. Photograph of the new TOFMS and existing laser system at SRI.

\subsubsection{Two-Color REMPI}

Most of the data shown in this report were recorded using the two-photon, one-color REMPI scheme where only the first photon is resonantly absorbed and the wavelength of the second photon is not important so long as it excites the molecule above the threshold for ionization. This scheme is possible only so long as the resonantly excited level lies at an energy of at least half of the ionization potential (IP). If the $S_{0} \rightarrow S_{1}$ transition energy is less than IP/2, then either a third photon will be required for ionization, or a second photon of higher energy is 
needed. Although three-photon REMPI is possible, it is less efficient than the two-photon scheme.

Figure 16 shows the variation in the $S_{0} \rightarrow S_{1}$ transition energy for some dioxin congeners as a function of their degree of chlorination. Although data are not available for most of the highly chlorinated dioxin congeners, estimates have been made based on conventional uv absorption spectra. A monotonic decrease in the transition energy with chlorination is predicted. Also shown in Figure 16 is the monotonic increase in IP/2 as a function of chlorination. For congeners that lie to the left of the crossing point of these two parameters, the one-color REMPI scheme can be used. This has been experimentally verified at least through the dichlorodibenzodioxin congener. Interestingly, the important tetrachloro-substituted dioxins lie very near the crossing point, and the applicability of the one-color REMPI scheme is uncertain. It is clear, however, that for more highly chlorinated congeners, a two-color scheme will be required.

In preparation for two-color REMPI studies, we assembled the necessary optical components to generate a second, fixed wavelength, of $266 \mathrm{~nm}$. Figure 17 shows the optical schematic of our modified laser system. Note that the tunable wavelength portion of the system remains essentially unchanged from the previously used configuration. The additional laser pulse at $266 \mathrm{~nm}$ is generated by doubling the excess $532 \mathrm{~nm}$ second harmonic of the Nd:YAG fundamental. This second wavelength pulse overlaps the tunable pulse spatially within the ion source; however, it is temporally delayed by $\sim 5 \mathrm{~ns}$. Although the delay is adjustable, $5 \mathrm{~ns}$ is a reasonable starting value. The delay must be long enough to allow molecules in resonance with the tunable light to be excited before being ionized by the $266 \mathrm{~nm}$ photon, yet not so long that they are either lost from the ionization region or the molecule returns to the ground state.

The additional laser pulse at $266 \mathrm{~nm}$ is generated by doubling the excess $532 \mathrm{~nm}$ second harmonic of the Nd:YAG fundamental. This second wavelength pulse overlaps the tunable pulse spatially within the ion source; however, it is temporally delayed by $\sim 5 \mathrm{~ns}$. Although the delay is adjustable, $5 \mathrm{~ns}$ is a reasonable starting value. The delay must be long enough to allow molecules in resonance with the tunable light to be excited before being ionized by the $266 \mathrm{~nm}$ photon, yet not so long that they are either lost from the ionization region or the molecule returns to the ground state.

\subsection{REMPI SPECTRA AND CALIBRATION METHODS}

The initial test experiments designed to verify operation of the jet-REMPI system consisted of placing a small amount of liquid 2,5-dichlorotoluene into the inlet line of the pulsed valve. The room temperature vapor pressure of 2,5-dichlorotoluene is high enough that no 


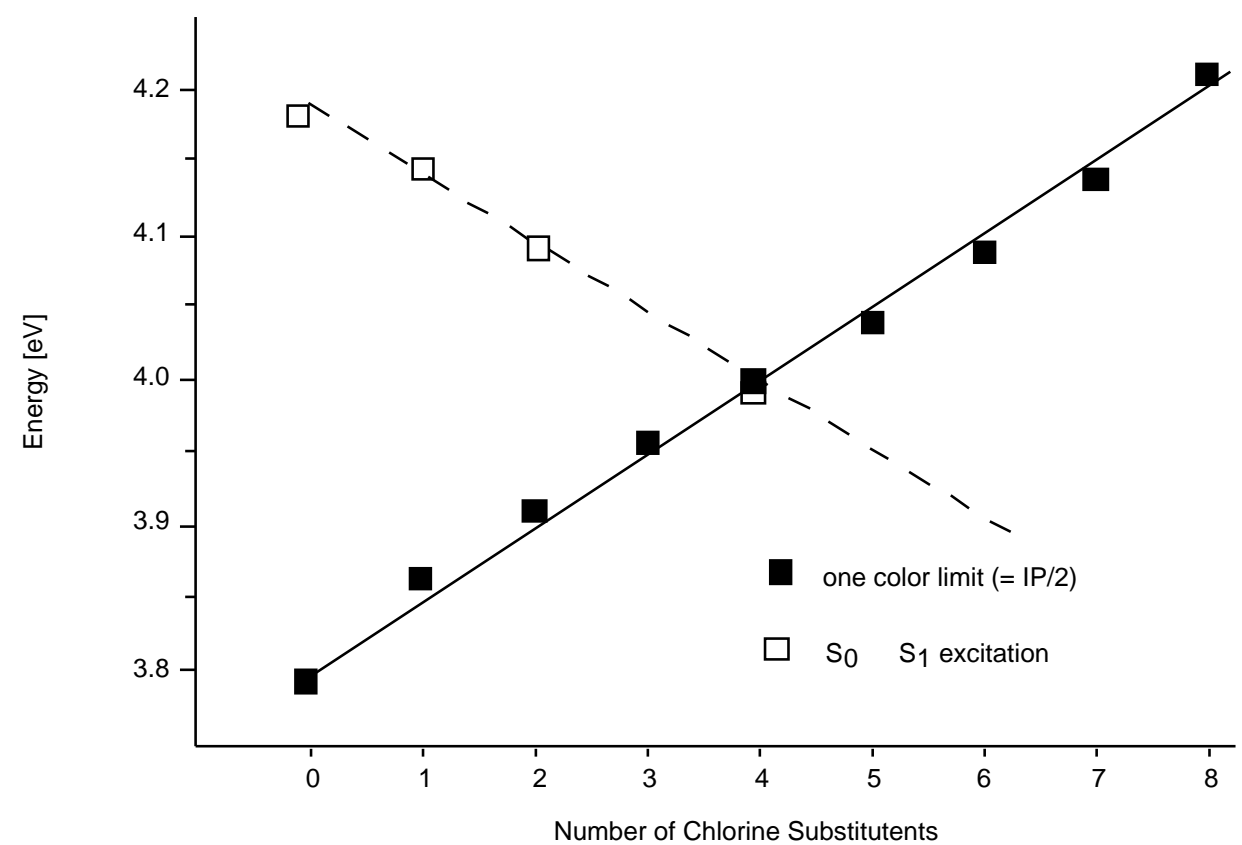

Figure 16. Variation of the S0 $\rightarrow \mathrm{S} 1$ excitation energy (o) as a function of chlorination for dibenzodioxins. Also shown is the one-color, two-photon ionization limit (n) corresponding to one half of the ionization potential. The $\mathrm{S} 0 \rightarrow \mathrm{S} 1$ excitation energy for the tetrachlorinated dibenzodioxin is estimated from conventional uv absorption spectroscopy.

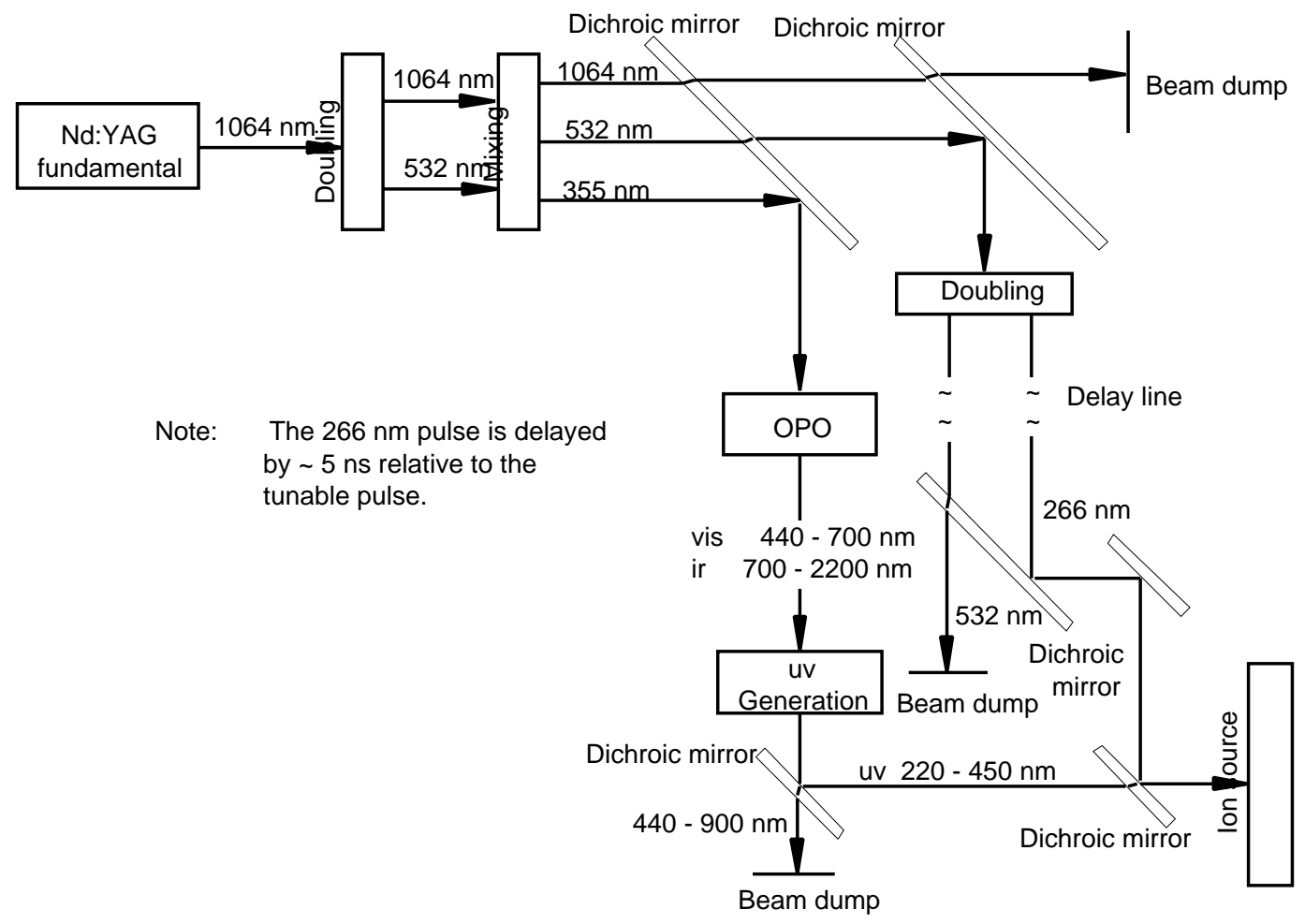

Figure 17. Two-photon, two-color REMPI optical scheme. 
heating was required to generate a sample. Unfiltered room air was used as a carrier gas in these experiments. Voltages on the TOFMS were initially adjusted according to the SIMION calculations, and the laser was tuned to a wavelength of $279.6 \mathrm{~nm}$ where strong REMPI absorption was expected. Within a short time, a mass spectrometric signal corresponding to the parent ion at 160 atomic mass units (amu) was observed. Some additional adjustments in the TOFMS voltages were made along with adjustments in the pulsed valve parameters and the delay time between the sample injection and the laser trigger.

We quickly found that the signal levels were too strong, causing nonlinear effects and saturation of the detection electronics. The signal levels were reduced by using a combination of reduced gain on the multichannel plate detector and by attenuating the laser intensity.

\subsubsection{Initial Test Spectra}

The initial mass spectrum measured for 2,5-dichlorotoluene showed very good mass resolution in excess of $500(\mathrm{~m} / \Delta \mathrm{m})$, and the chlorine isotopic peaks were easily resolved, as shown in Figure 18. In addition, no significant fragmentation was observed, and no extraneous background peaks were present. In this mass spectrum, the three major isotopic peaks at $\mathrm{m} / \mathrm{z}$ 160,162 , and 164, corresponding to ${ }^{35} \mathrm{Cl}^{35} \mathrm{Cl},{ }^{35} \mathrm{Cl}^{37} \mathrm{Cl}$, and ${ }^{37} \mathrm{Cl}^{37} \mathrm{Cl}$, respectively, are in the ratio of 100:66.7:10.4. While these ratios are very close to the expected values of 100:65.0:10.6, the minor discrepancies are actually the result of the very high wavelength resolution of our instrument and the slight shift in resonance energy associated with the $1 \%$ mass difference among the different isotopic species. Thus, a wavelength of $279.6 \mathrm{~nm}$ will be in exact resonance with the molecules containing ${ }^{35} \mathrm{Cl}^{35} \mathrm{Cl}$, slightly off resonance with the molecules containing ${ }^{35} \mathrm{Cl}^{37} \mathrm{Cl}$, and even further off resonance with the ${ }^{37} \mathrm{Cl}^{37} \mathrm{Cl}$ species.

A wavelength scan was performed for 2,5-dichlorotoluene between 260 and $285 \mathrm{~nm}$. The resulting REMPI signal at m/z 160 is shown in Figure 19. This preliminary jet-REMPI data indicated that the molecules had not achieved the desired degree of internal cooling. This was manifested as wider than expected peaks in the wavelength dependant scan of the parent mass ions shown in Figure 19. While the wavelength dependence had the expected peaks at the correct locations, the peak widths were considerably larger $(50 \%)$ than expected from previously published results on the same molecule. Two possible factors can lead to the observed peak broadening: a wider than expected laser linewidth, or target molecules that have retained excessive internal energy as a result of insufficient cooling in the gas expansion. Since the $\mathrm{Nd}$ :YAG-OPO system was operating optimally with a linewidth on the order of $0.04 \mathrm{~cm}^{-1}$, the most obvious source of peak broadening was due to a lack of gas cooling in the jet. 


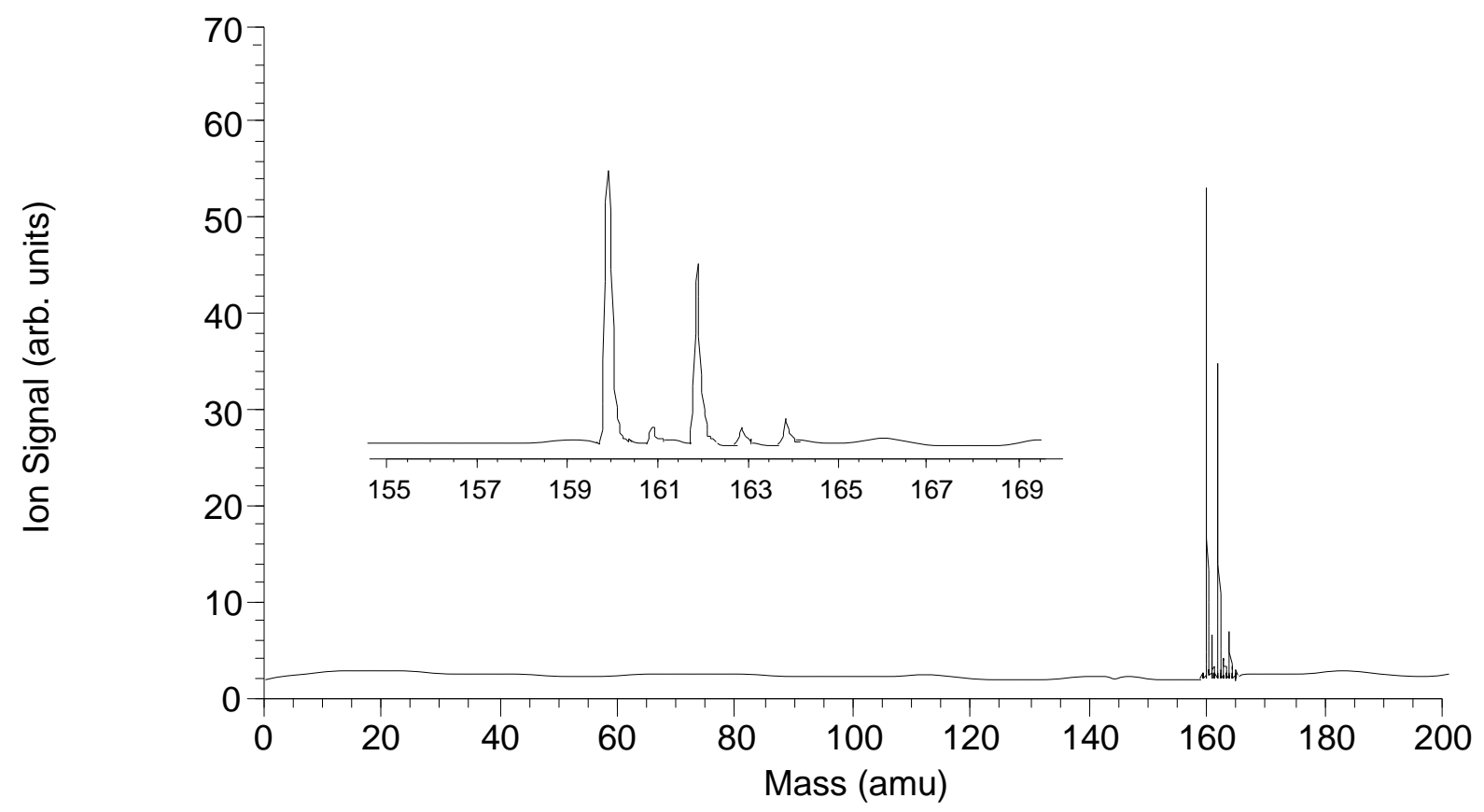

Figure 18. Mass spectrum recorded for jet-REMPI detection of 2,5-dichlorotoluene at a wavelength of $279.6 \mathrm{~nm}$. The inset shows an expanded region about the parent ion peaks. The chlorine isotope peaks are clearly resolved.

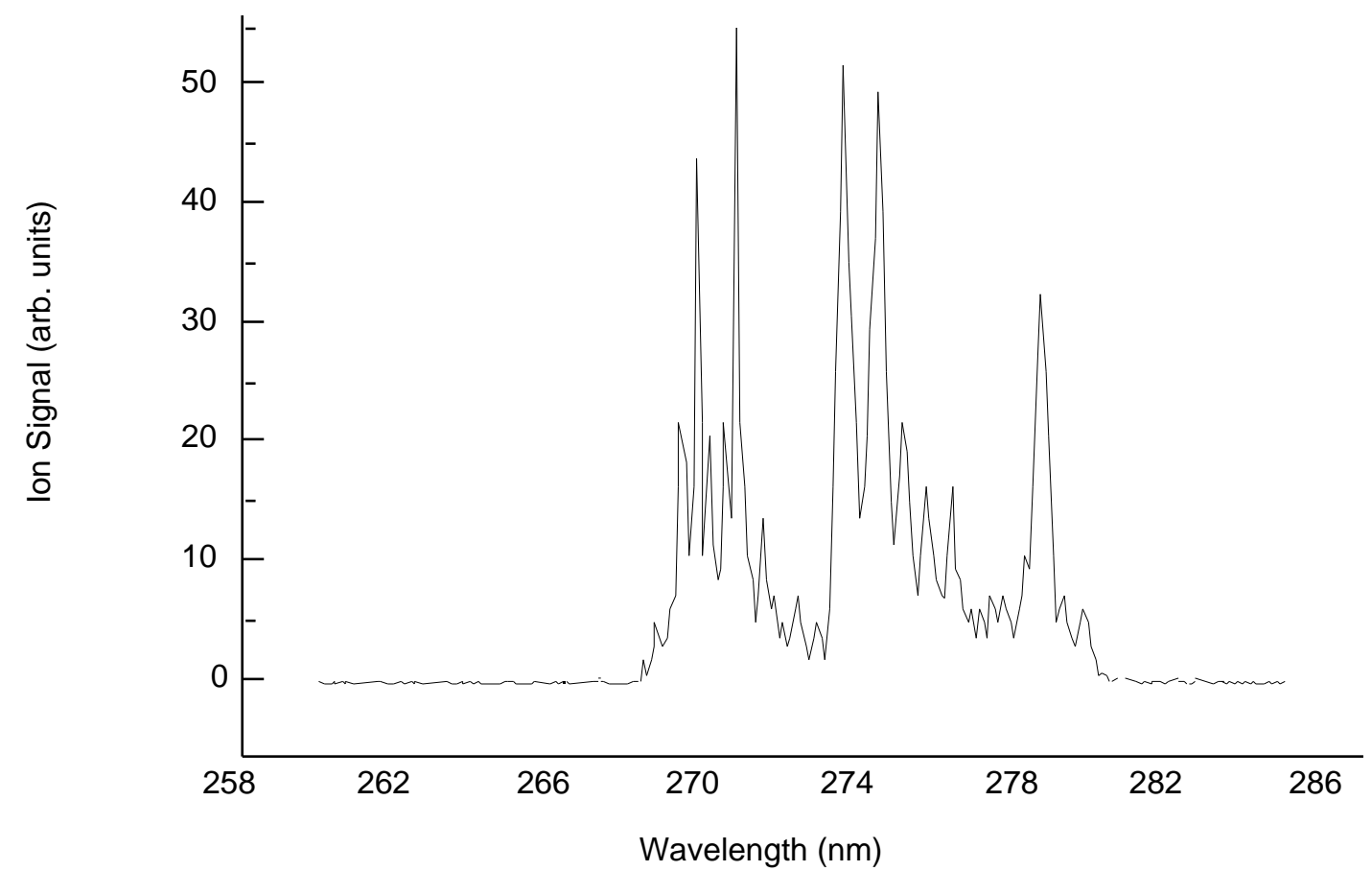

Figure 19. Wavelength dependence of the $\mathrm{m} / \mathrm{z} 160$ ion signal for jet-REMPI detection of 2,5dichlorotoluene.

Molecules that have undergone a free-jet expansion will not exhibit the expected degree of internal cooling if they have undergone either too few collisions during the expansion or too 
many collisions after the expansion. Given our operating conditions, the valve timing parameters, and system pressures, it was apparent that the most reasonable explanation was too many collisions. Also, during our testing, first one and then the second of our $250 \mathrm{~L} / \mathrm{s}$ turbomolecular pumps failed. The pump on the flight tube region was temporarily replaced with a $170 \mathrm{~L} / \mathrm{s}$ pump, while the source chamber pump was temporarily replaced with a $330 \mathrm{~L} / \mathrm{s}$ turbomolecular pump.

With this configuration, we observed a slight narrowing of the absorption peaks, but still not to the degree expected. We did, however, observe that the pressure differential between the source (ionization) chamber and the flight tube was only a factor of 3 , indicating that too much of the sample gas load was entering the flight tube, rather than being retained and removed in the source chamber. This observation led to a change in the way we isolated the two vacuum regions. Specifically, we incorporated a cylindrical metal can that enclosed the ion extraction optics. This isolation can was sealed to the ion lenses, using a closely fitting Teflon ring.

Later tests showed a slightly improved vacuum isolation; however, the relatively large (1.27-cm-diameter) aperture in the ion extraction plate still provided too much gas conductance. Because the ion beam at this position is expected to be a few millimeter in diameter, this aperture size was reduced to $0.63 \mathrm{~cm}$ in diameter. The combination of enclosing the ion optics with the smaller extraction aperture produced more than an order of magnitude reduction in the pressure differential between the two chambers. Under these conditions, we again measured the wavelength dependence for detection of 2,5-dichlorotoluene and found the peaks to be as narrow as previously reported, indicating that the desired degree of molecular cooling had been achieved.

The desired cooling effect was verified by measuring the wavelength dependence for both 1,2-dichlorobenzene and monochlorobenzene. Both molecules exhibited very narrow resonance absorption peaks. These results are shown in Figures 20 and 21, respectively. Figure 22 shows the mass spectrum measured for the jet-REMPI detection of 1,2dichlorobenzene at a wavelength of $261.2 \mathrm{~nm}$. Again, only the parent ion signals, including isotopic chlorine, are observed. Similarly, Figure 23 displays the mass spectrum measured for the jet-REMPI detection of monochlorobenzene at a wavelength of $266.0 \mathrm{~nm}$. 


\section{Draft}

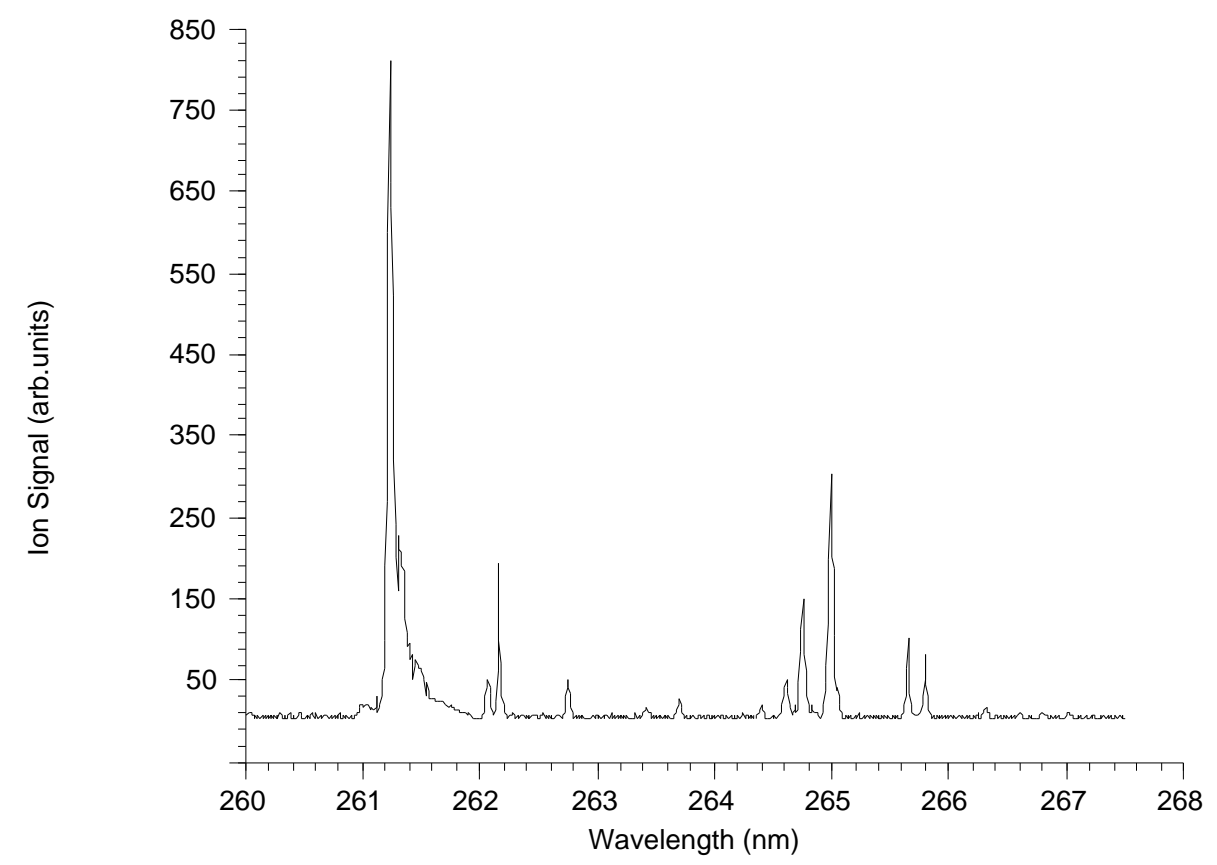

Figure 20. Wavelength dependence of the $\mathrm{m} / \mathrm{z} 146$ ion signal for the jet-REMPI detection of 1,2dichlorobenzene.

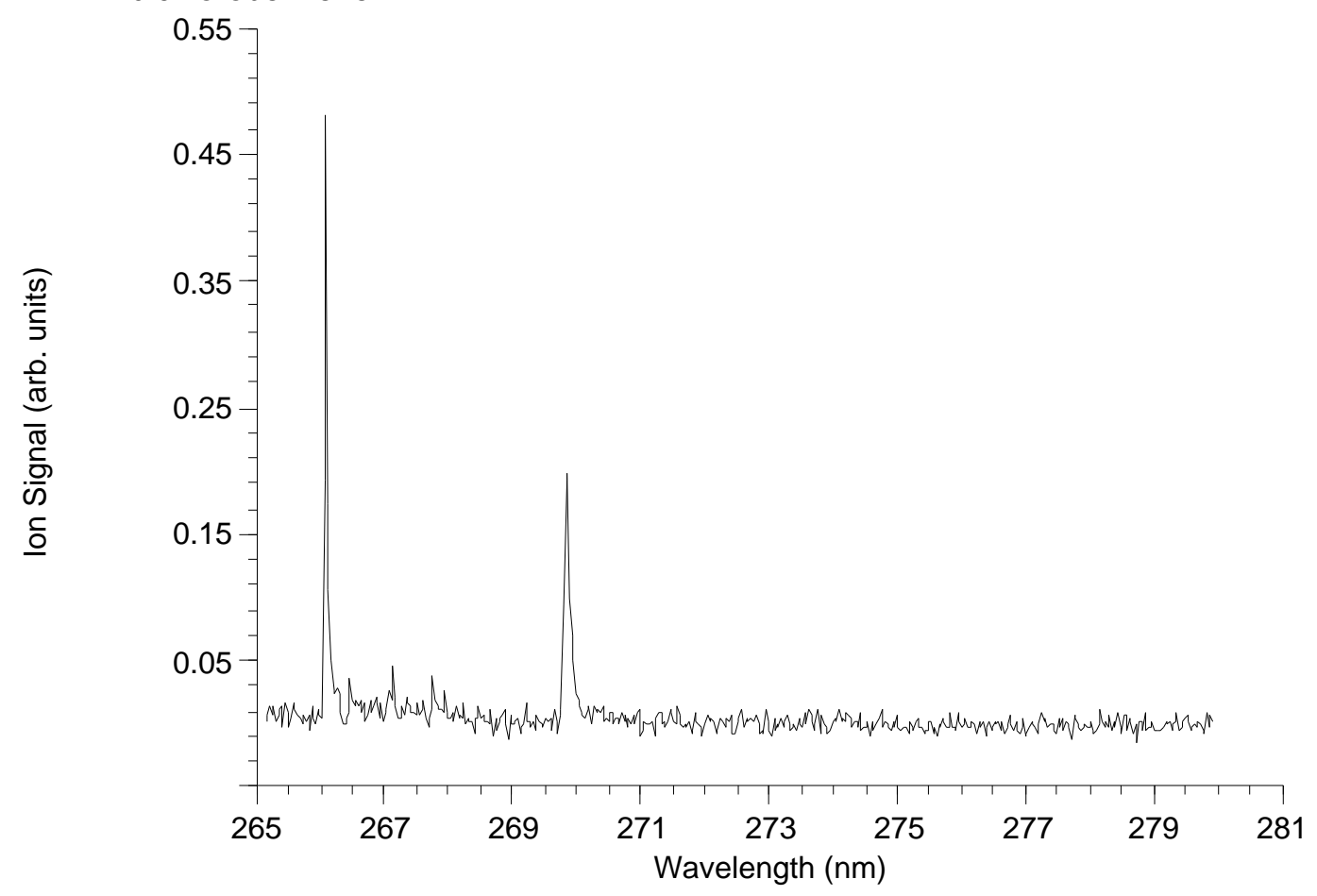

Figure 21. Wavelength dependence of the $\mathrm{m} / \mathrm{z} 112$ ion signal for the jet-REMPI detection of monochlorobenzene. 


\section{Draft}

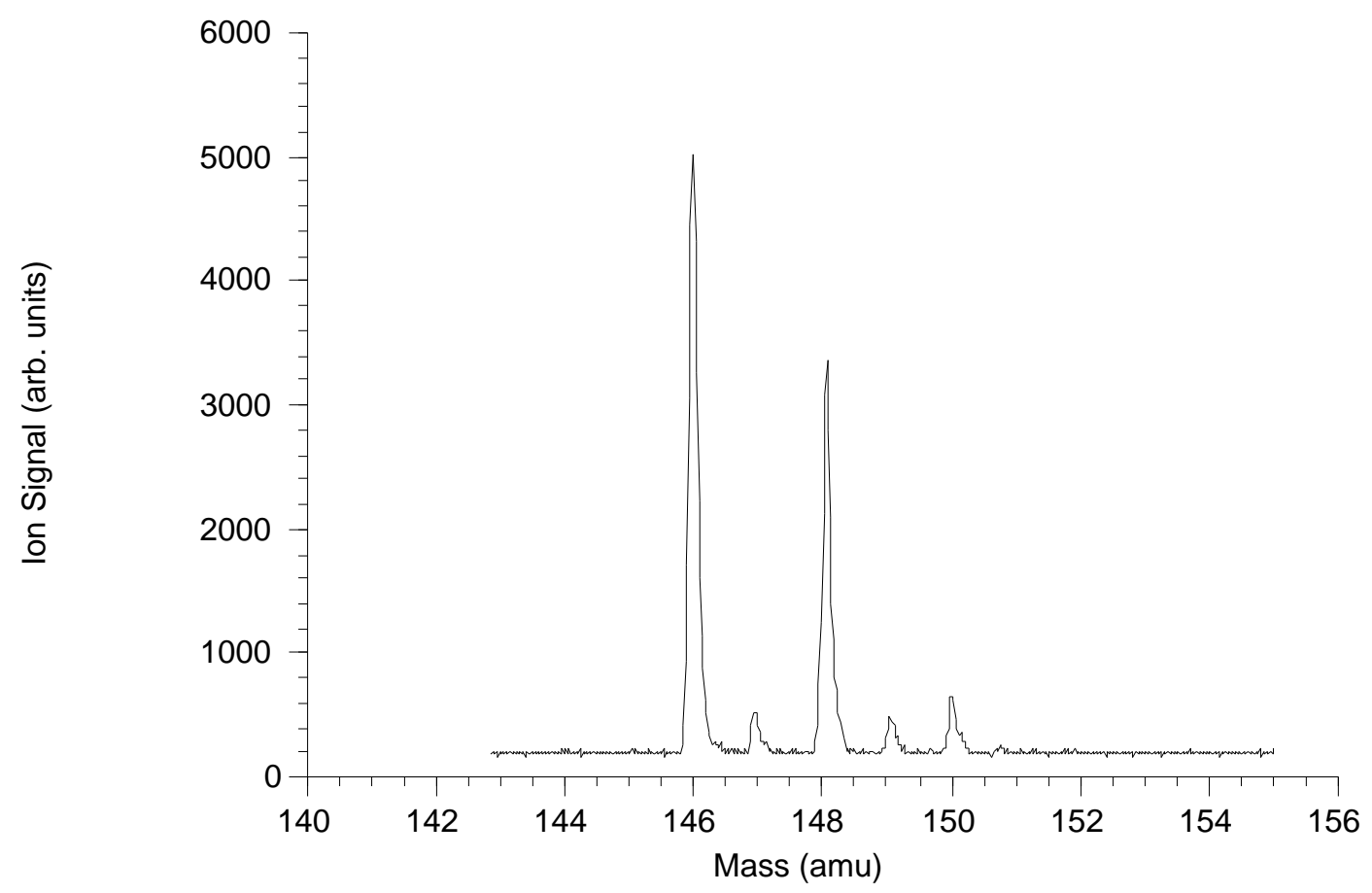

Figure 22. Mass spectrum recorded for jet-REMPI detection of 1,2-dichlorobenzene at wavelength of

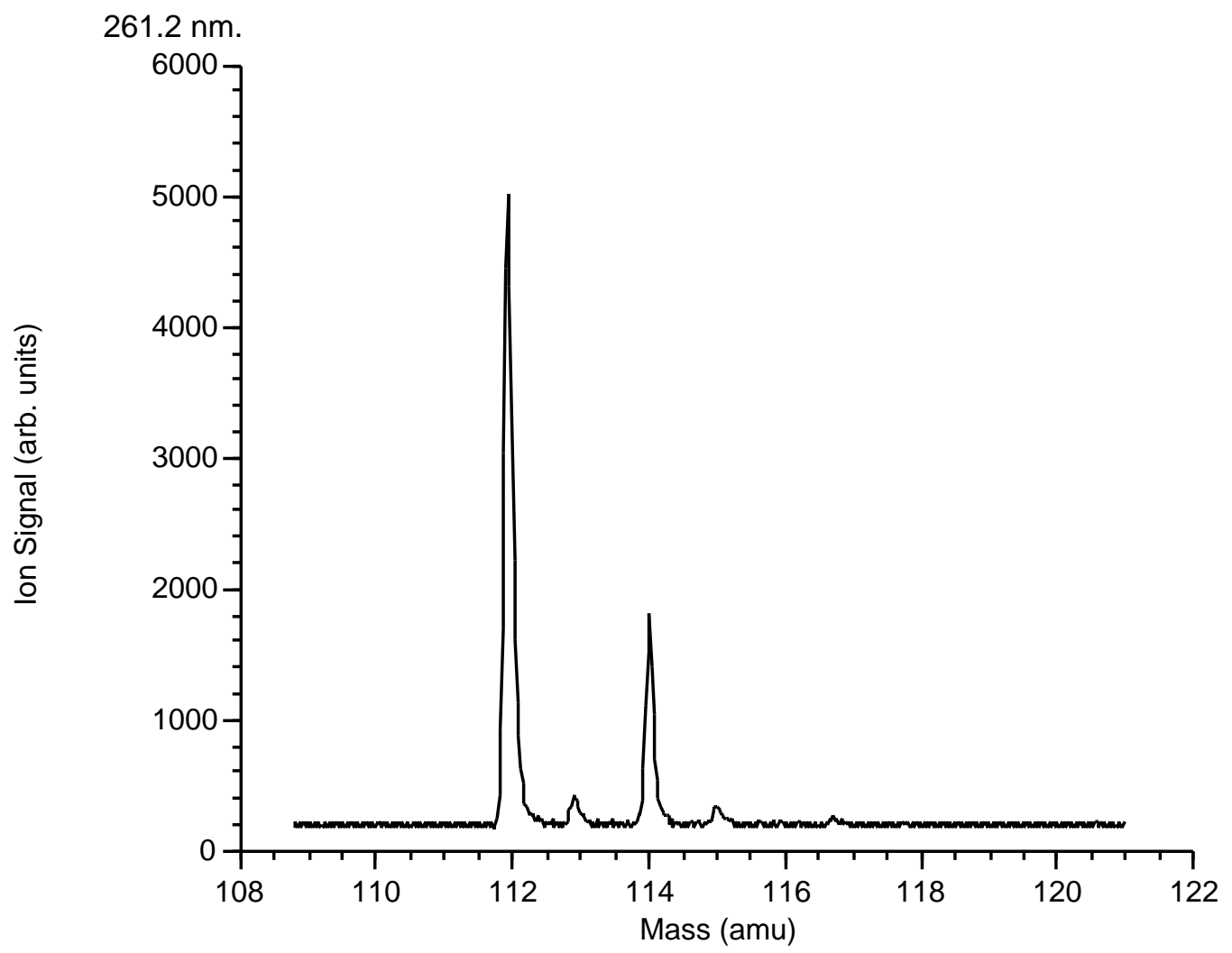

Figure 23. Mass spectrum recorded for jet-REMPI detection of monochlorobenzene at wavelength of $266.0 \mathrm{~nm}$. 
Following the source modifications described above, the jet-REMPI instrument appeared to provide the expected degree of both optical resolution and mass resolution. These studies clearly indicate the importance of maintaining the optimum vacuum conditions within the ionization and ion extraction regions. They also indicate that the $250 \mathrm{~L} / \mathrm{s}$ turbomolecular pump used initially on the source region was too small to adequately handle the gas load from the pulsed nozzle, especially for opening times of $200 \mu$ s or greater. Fortunately, larger pumps are readily available and can easily be incorporated into the system without any modifications. Larger pumps will become more important when the new valve is used due to the higher gas flow.

Our first tests showed that the smaller aperture in the ion extract electrode produced the desired reduction in the pressure differential between the ionization and detection chambers. We also found that the wavelength dependence for detection of 2,5-dichlorotoluene showed peaks that were as narrow as previously reported. This verified that the desired degree of molecular cooling had been achieved. However, it remained to verify that the signal levels (and hence, the sensitivity) of the instrument had not been adversely affected by the smaller aperture.

We therefore measured the signal levels for both 1,2-dichlorobenzene, and monochlorobenzene. With some minor adjustment of the ion optical potentials, we found the signal levels to be essentially the same as those previously measured with the larger aperture. This finding is consistent with our expectation that the ion beam is smaller in diameter than the $0.63-\mathrm{cm}$ aperture.

During this work, which involved the use of 1,2-dichlorobenzene as the sample gas, we observed the expected, but very small, isotope shift in the peak optical absorption wavelength due to ${ }^{37} \mathrm{Cl}$ relative to ${ }^{35} \mathrm{Cl}$. This effect was observed by changing the OPO wavelength by $0.005 \mathrm{~nm}$ and observing that the ion signal associated with the ${ }^{35} \mathrm{Cl}^{35} \mathrm{Cl}_{-}-\mathrm{C}_{6} \mathrm{H}_{4}$ decreased while the corresponding signal from the ${ }^{37} \mathrm{Cl}^{37} \mathrm{Cl}-\mathrm{C}_{6} \mathrm{H}_{4}$ increased. Our ability to observe this effect confirms several experimental factors. The OPO wavelength is very stable and reproducible, and the sample molecules have achieved a high degree of internal cooling, allowing for very narrow absorption features.

\subsubsection{Lowly Chlorinated Dioxins}

Following our preliminary experiments on chlorinated benzene and toluene that established the operational ability of our instrument, we began an initial survey of the jet-REMPI spectra for simple, lowly chlorinated dioxins. Three congeners were examined in pure vapor form: 2-monochlorodibenzodioxin, 2,7-dichlorodibenzodioxin, and 2,8-dichlorodibenzodioxin. 
REMPI spectra for each of these compounds have been reported previously by others. We also studied, a mixture of the 2,7-dichlorodibenzodioxin, and 2,8-dichlorodibenzodioxin congeners. This is the first time that a mixture of these two closely related dioxin congeners has been measured using jet-REMPI.

Figures 24 through 26 show the measured jet-REMPI wavelength dependence for the three dioxin congeners studied in pure vapor form. For each congener, note that the ion signal shows numerous, narrow, absorption peaks corresponding to resonant excitation of different rovibrational levels in the ground electronic state of each molecule. Furthermore, there is no background ion signal measured at off-resonance wavelengths. For each congener, the corresponding mass spectrum shows only the expected parent ion peaks, including the chlorine isotopic peaks.

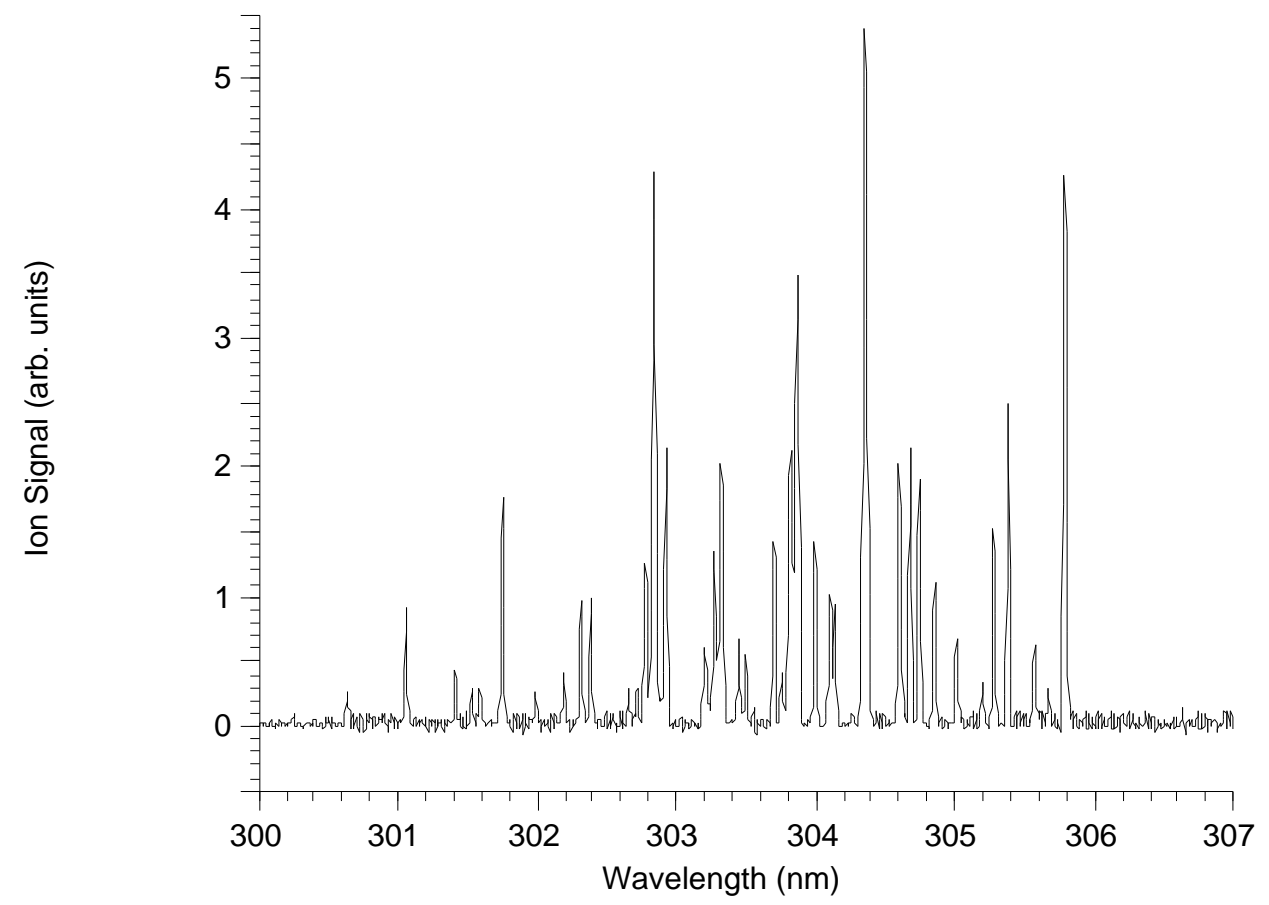

Figure 24. Wavelength dependence of the $\mathrm{m} / \mathrm{z} 218$ ion signal for the jet-REMPI detection of 2monochlorodibenzodioxin. 


\section{Draft}

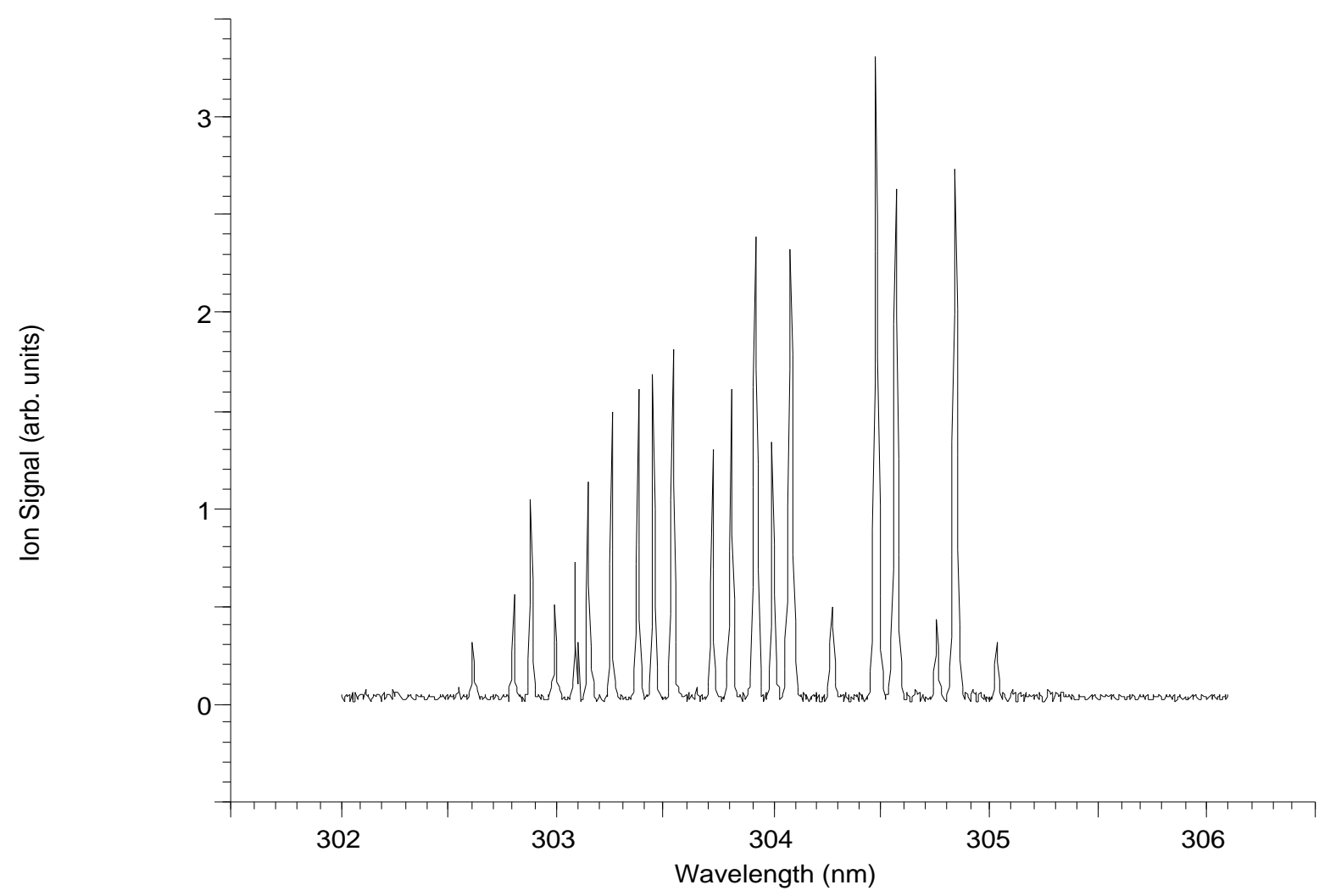

Figure 25. Wavelength dependence of the $\mathrm{m} / \mathrm{z} 252$ ion signal for jet-REMPI detection of 2,7-

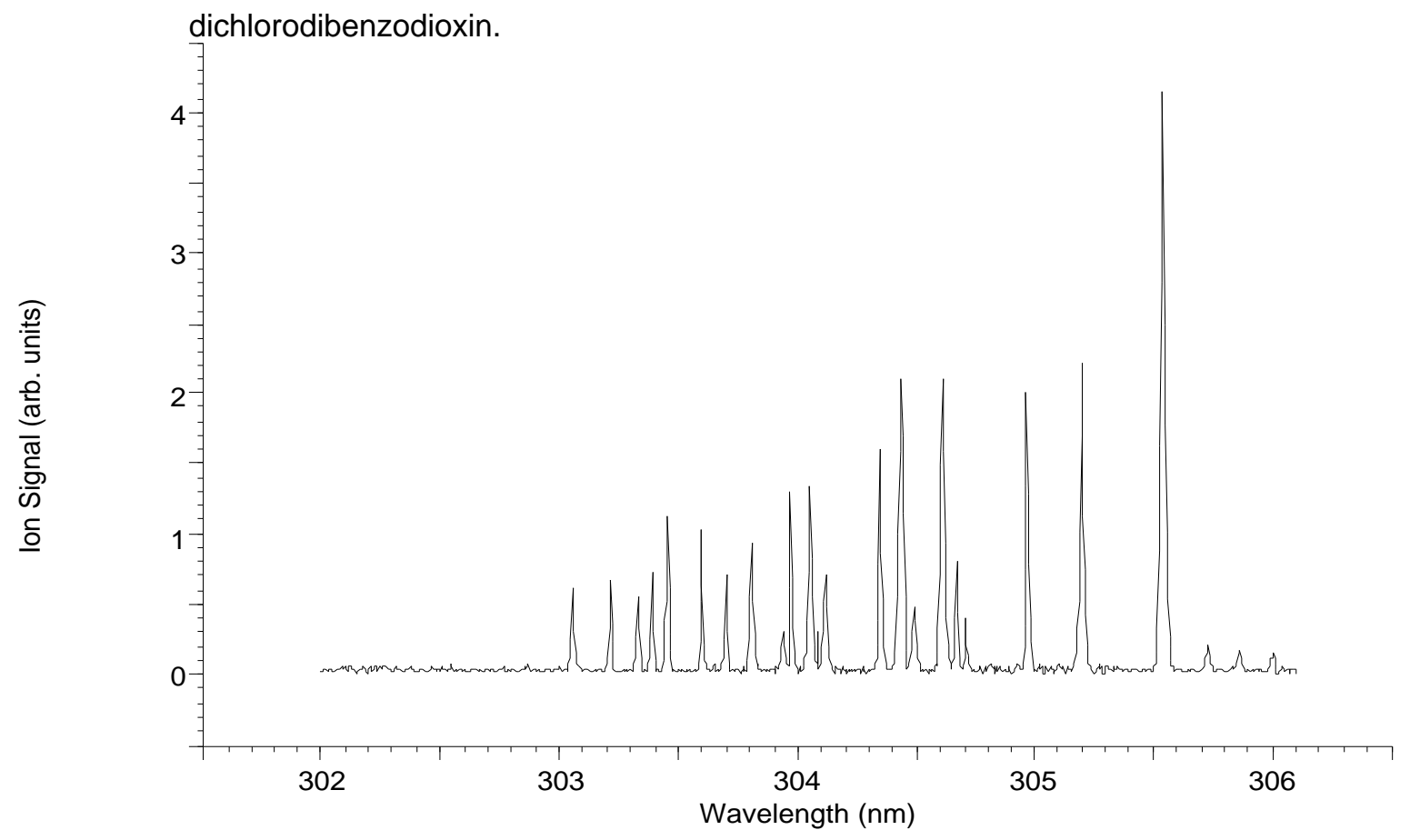

Figure 26. Wavelength dependence of the $\mathrm{m} / \mathrm{z} 252$ ion signal for jet-REMPI detection of 2,8dichlorodibenzodioxin. 
Of particular interest and importance are the data shown in Figure 27. Here, the sample consisted of an equimolar mixture of the 2,7- and 2,8-dichlorodibenzodioxin congeners. Several absorption peaks corresponding to each of the congeners have been noted to show that many wavelengths are available for separately detecting these two closely related congeners in a mixture. The mixture data in Figure 27 are essentially the algebraic sum of the wavelength scans for the separate component shown in Figures 25 and 26. Although it has always been presumed that such selective detection of closely related congeners was possible using this method, this is the first clear demonstration.

\subsubsection{Other REMPI Spectra}

During the course of Phase 2, we measured the wavelength-dependent REMPI spectra of a number of important HAPs that are likely present in most incinerator exhaust streams. Two examples are shown in Figures 28 and 29. Figure 28 shows the wavelength dependence for the jet-REMPI detection of p-xylene, while Figure 29 shows the corresponding data for phenol.

As part of our development of a rapid calibration method, our new data acquisition software is capable of recording the full mass spectrum as the wavelength is varied. The result of this can be viewed as a three-dimensional, false-color map showing the ion intensity (color) as a function of the mass (y-axis) and wavelength (x-axis). An example of this type of map is shown in Figure 30. Here, a mixture of benzene (78 amu), toluene (92 amu), and chlorobenzene

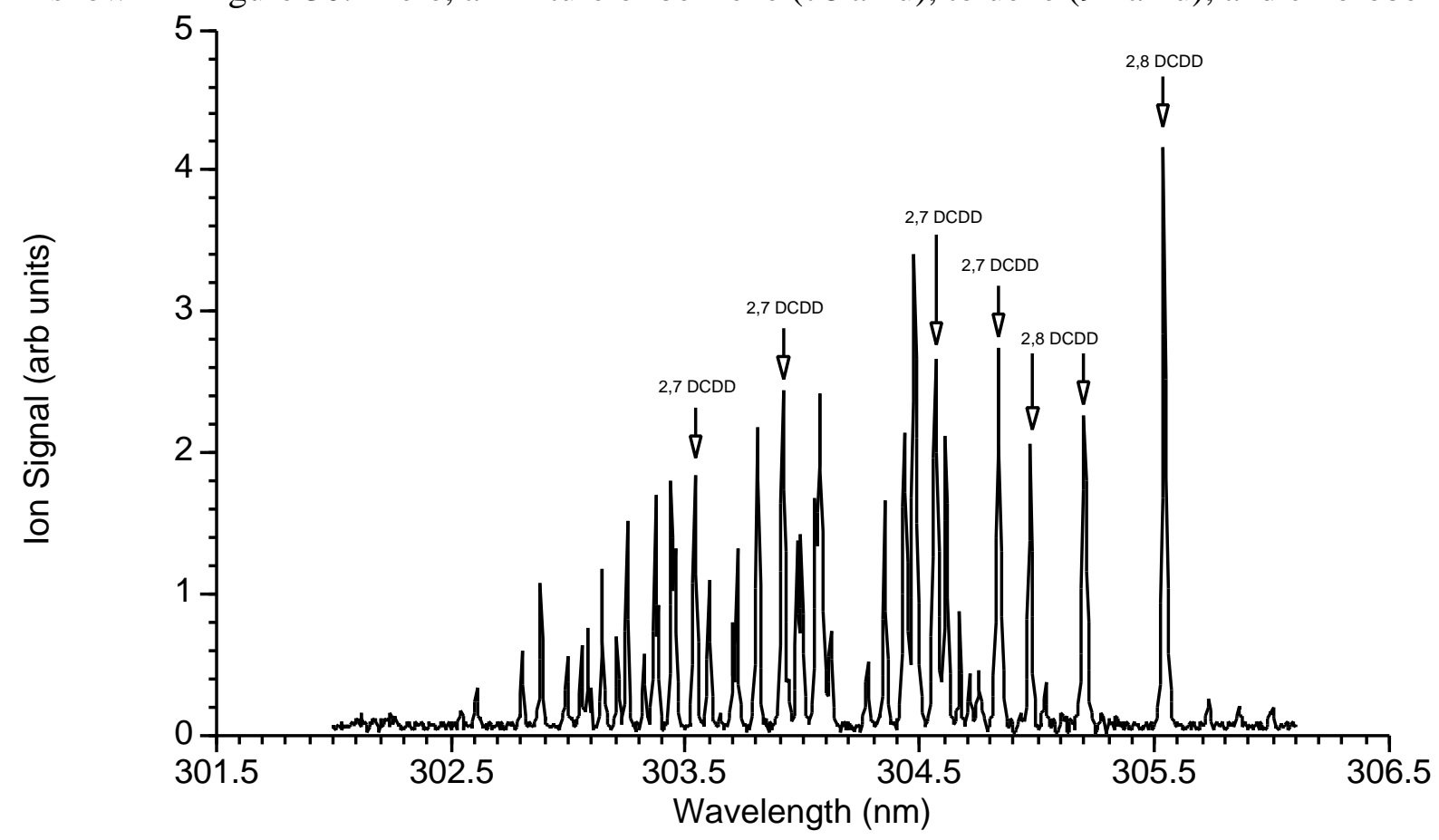

Figure 27. Wavelength dependence of the ion signal at $\mathrm{m} / \mathrm{z} 252$ for the jet-REMPI detection of a mixture of 2,7-dichlorodibenzodioxin and 2,8-dichlorodibenzodioxin. 


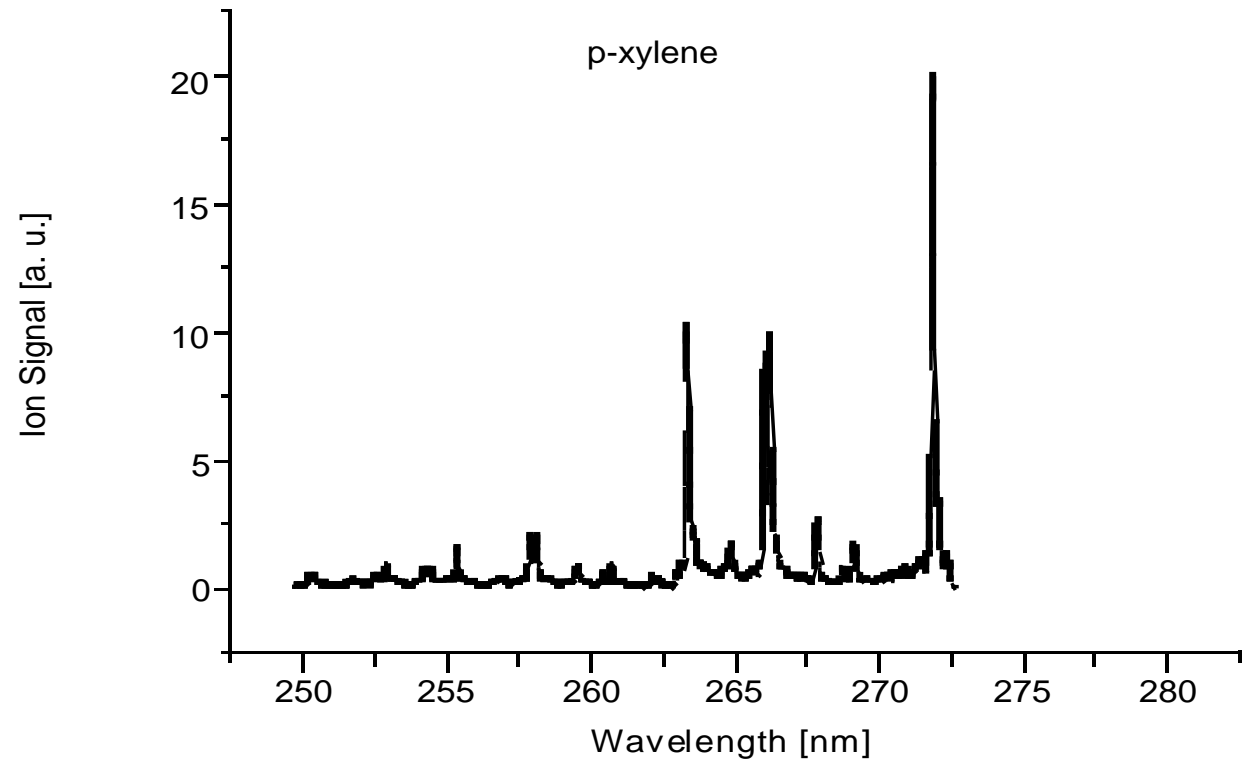

Figure 28. REMPI excitation spectrum for $p$-xylene.

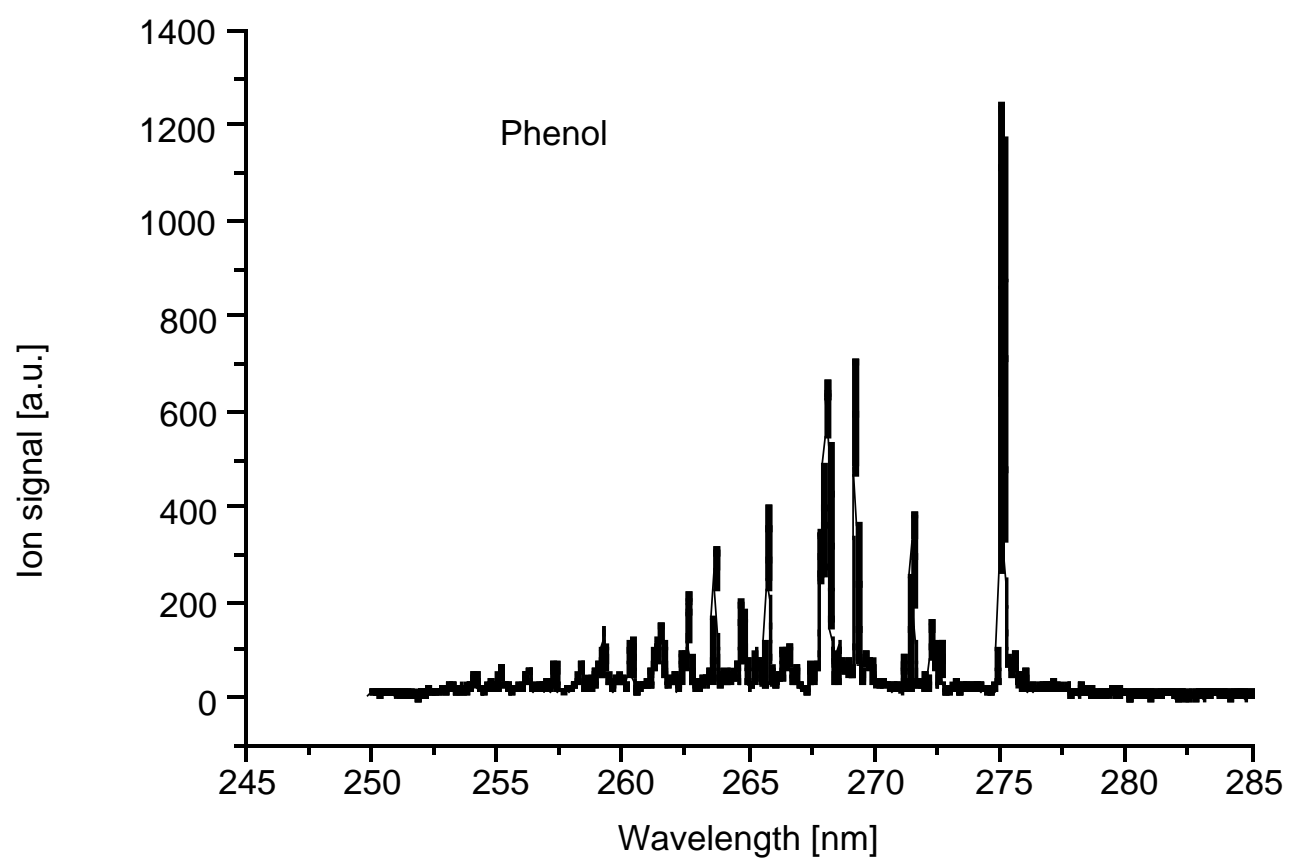

Figure 29. REMPI excitation spectrum for phenol.

(112 amu) diluted in room air was used as the sample. It is apparent from Figure 30 that each compound only gives rise to a single parent ion signal, however, that ion signal appears at a number of wavelengths. By taking a cut in the vertical direction at a fixed wavelength, you would produce a mass spectrum showing what ion signals you would expect if all of these compounds were present in the sample stream; that is, a chemical interference spectrum. 
By taking the corresponding horizontal cut through the data at a fixed mass, you would obtain the normal REMPI excitation spectra, such as those shown in Figures 28 and 29. It must be noted that the results shown in Figure 30 are preliminary in nature and included here only to demonstrate the type of data visualization display that is being developed.

As an example, Figure 31 shows three horizontal cuts in the data from Figure 30, one at each of the three primary mass peaks. As expected, we easily recover the REMPI excitation spectra for the individual compounds even though only one set of data was recorded.

Two additional test compounds were investigated, aniline, and o-toluidine. Both compounds are known to yield sharp, well-defined REMPI spectra. By measuring these spectra, we can simultaneously verify the wavelength dependence, the absorption linewidths, the absolute mass calibration, and the mass resolution. The spectra that were measured for each compound are shown in Figures 32 and 33. These spectra were indistinguishable from those that we previously measured with the older instrument, as well as spectra reported in the literature measured under comparable conditions.

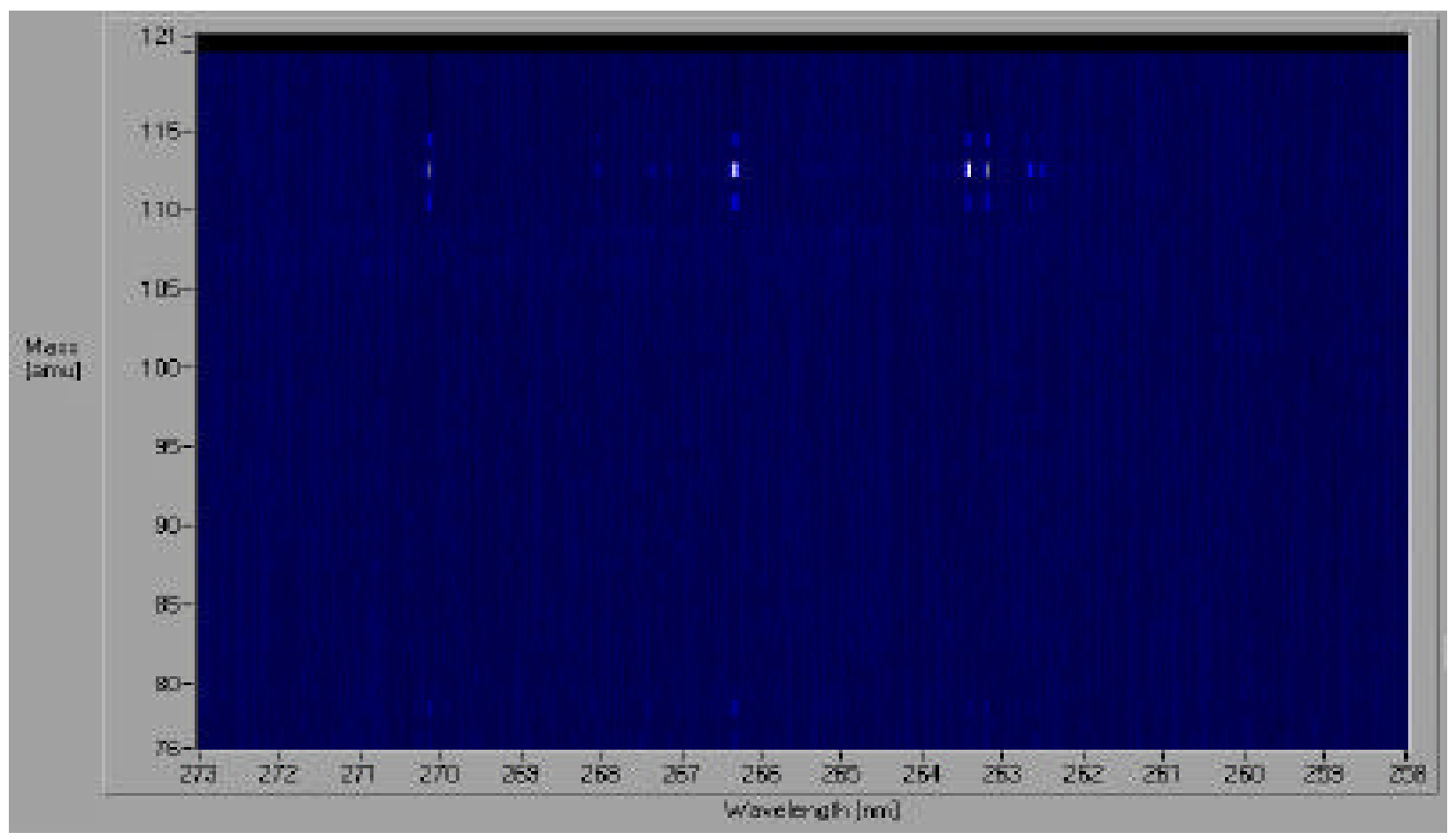

Figure 30. False color map showing the intensity (color) measured as a function of wavelength and mass for a mixture of benzene (78 amu), toluene (92 amu), and chlorobenzene (112 amu). 


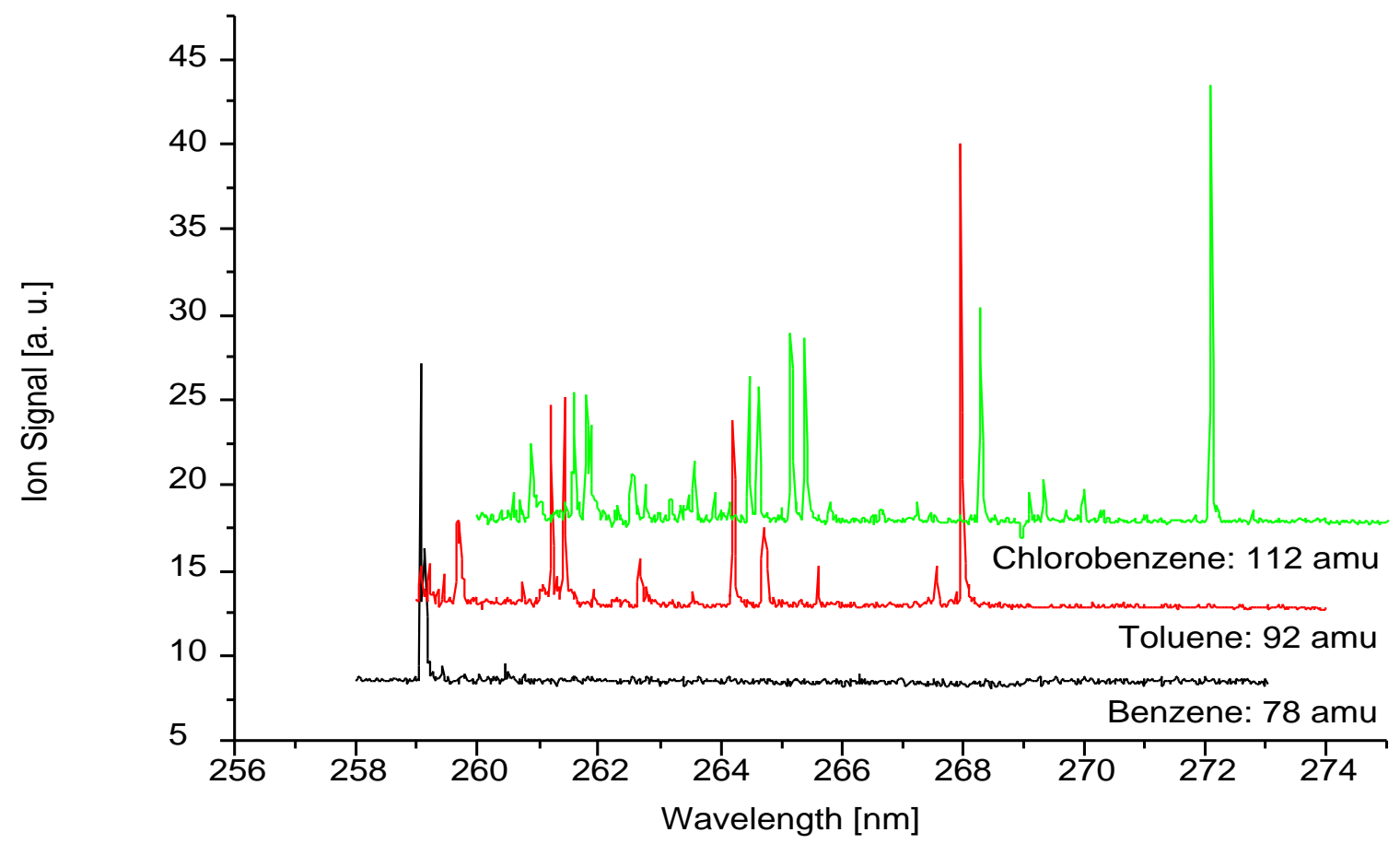

Figure 31. REMPI excitation spectra for benzene, toluene, and chlorobenzene derived from the threedimensional map in Figure 30.

Ion Signal [a. u.]

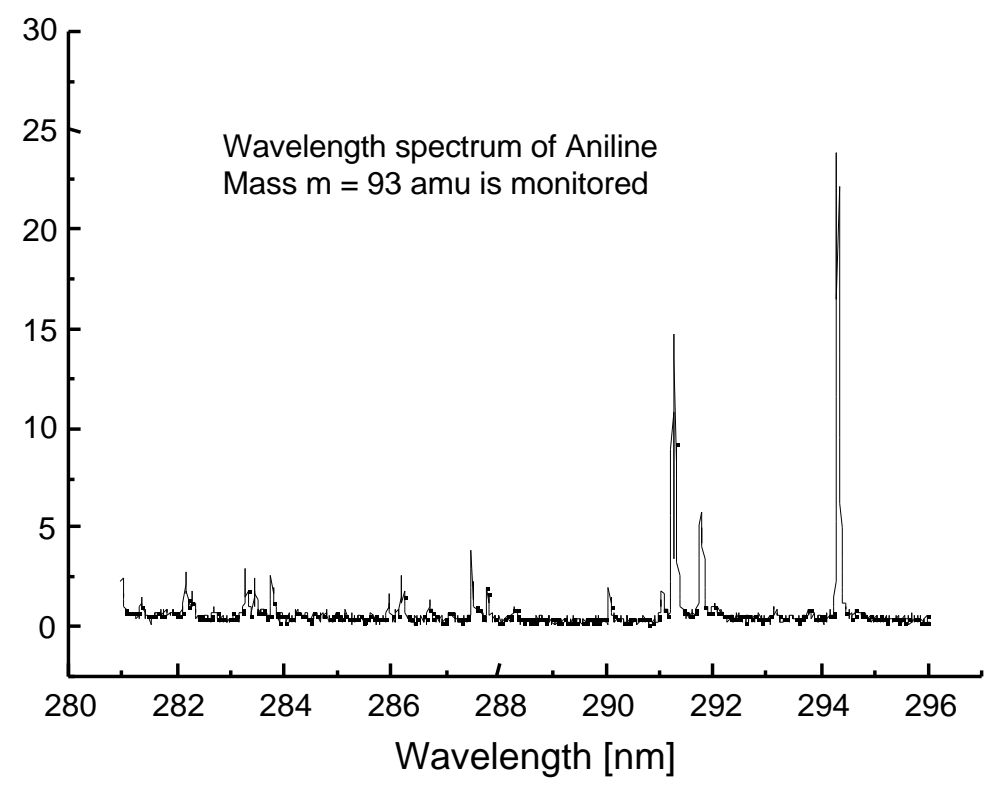

Figure 32. Wavelength dependence for the REMPI ionization of aniline. 


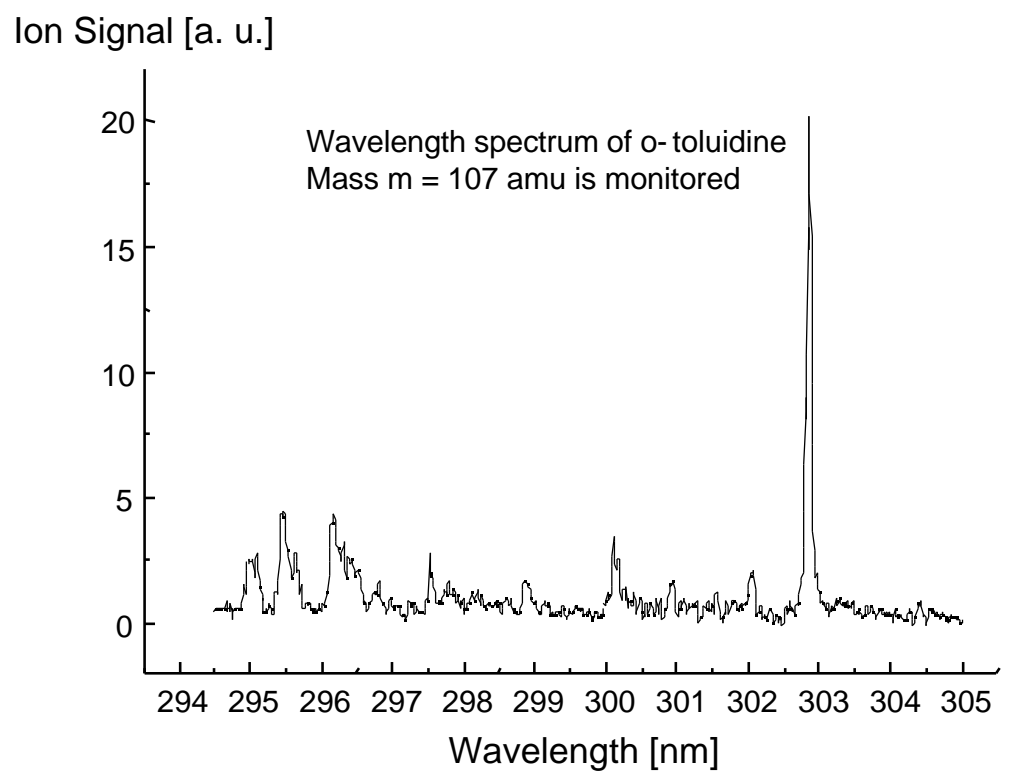

Figure 33. Wavelength dependence for the REMPI ionization of o-toluidine.

Based on the results obtained with the new mass spectrometer, we investigated the REMPI spectra of the unchlorinated dibenzodioxin. Our wavelength scan is shown in Figure 34.

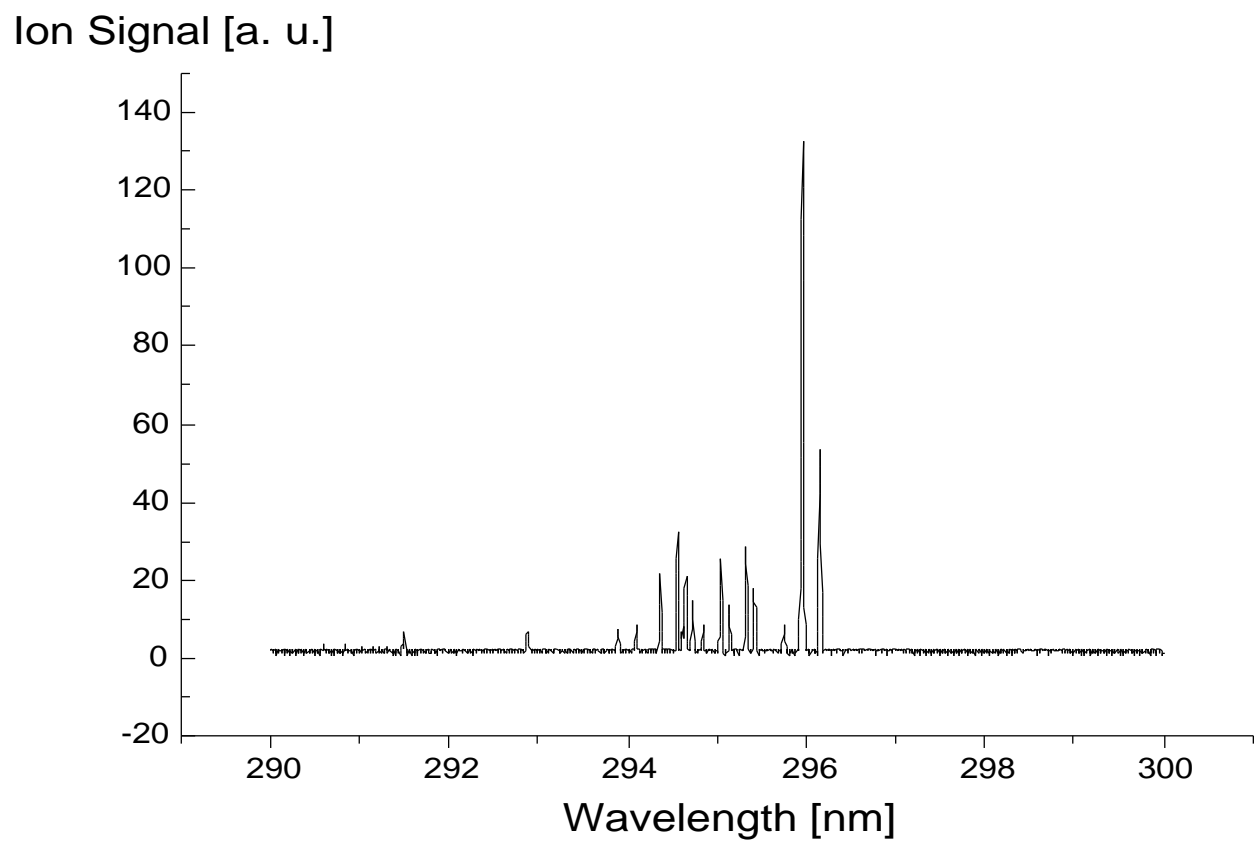

Figure 34. Wavelength dependence for the REMPI ionization of dibenzodioxin.

Although the spectra measured using the new mass spectrometer system, including aniline, o-toluidine, and unchlorinated dibenzodioxin, shown in Figures 32, 33, and 34, 
respectively, were essentially the same as we previously had measured with the older instrument, we soon found that for even lowly chlorinated compounds with moderate vapor pressures, the REMPI spectra were much broader than expected. The apparent cause for this poor optical resolution was insufficient thermal cooling in the jet expansion. We had previously observed similar effects when using the original ion extraction configuration as supplied by R. M. Jordan. Since the new spectrometer incorporated the standard Jordan source, we concluded that the small spacing between the repeller and extraction electrodes was responsible for disrupting the free-jet expansion and thereby preventing sufficient collisional cooling. To quickly overcome this problem, we removed the ion source from our older spectrometer that had been modified to increase the electrode spacing, and installed it in our new spectrometer. We then verified that full molecular cooling was achieved.

As a test molecule, we examined the REMPI absorption spectrum of 2monochlorodibenzofuran (2-MCF). This species had not previously been studied by REMPI. Figure 35 shows the jet-REMPI signal recorded for the parent $\left({ }^{35} \mathrm{Cl}\right)$ ion at $\mathrm{m} / \mathrm{z} 202$ as a function of the wavelength for a one-color, two-photon ionization process. The wavelength dependence shows numerous, well-resolved, absorption features. We noted, however, that both he relatively low signal level and the lack of an apparent $S_{0} \rightarrow S_{1}$ band origin at long wavelengths might indicate that a two-color scheme would yield more sensitivity. We have made a very preliminary test of this by combining a tunable photon with a fixed $266 \mathrm{~nm}$ photon, and indeed we see an enhanced signal.

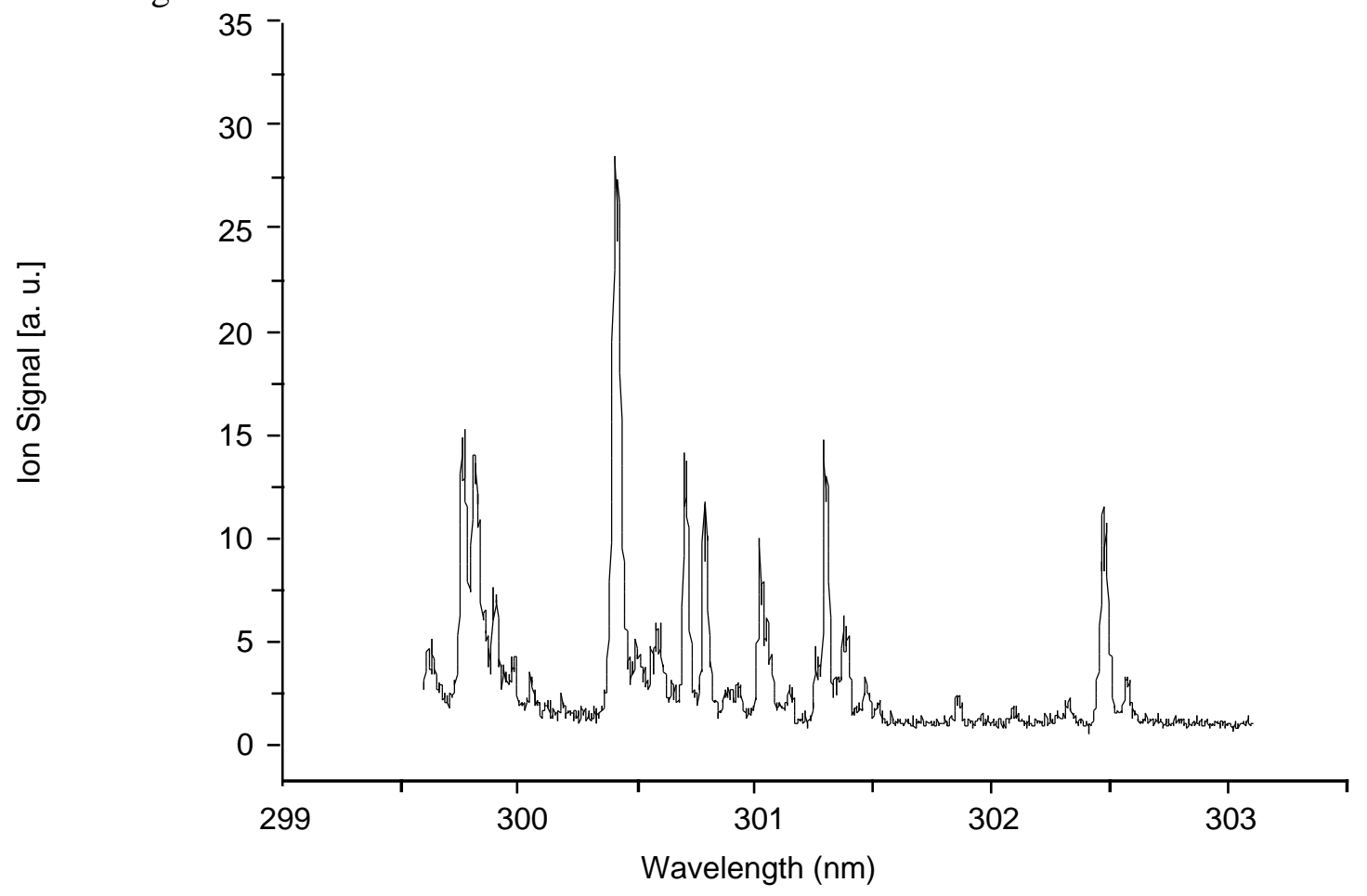

Figure 35. Wavelength dependence for the REMPI ionization of 2-monochlorodibenzofuran. 


\subsubsection{Two-Color REMPI Spectra}

To demonstrate the two-color approach, we again examined the jet-REMPI wavelength dependence for 1,2-dichlorobenzene. Figure 36 shows the $\mathrm{m} / \mathrm{z} 146$ ion signal measured using a combination of tunable uv from the OPO and $266 \mathrm{~nm}$ produced by quadrupling the $\mathrm{Nd}$ :YAG fundamental. Since 1,2-dichlorobenzene can also be ionized by the one-color, two-photon scheme, we have shown that ion signal in Figure 36 as well.

Note that the large absorption peak at $272.61 \mathrm{~nm}$ appears using both ionization schemes. However, the absorption peak at $273.58 \mathrm{~nm}$ appears only in the two-color signal. This feature arises from the absorption of one photon that is resonant with vibrationally excited ground state molecules, followed by absorption of a $266 \mathrm{~nm}$ photon to produce ionization. Because the absorption of a second photon at $273.58 \mathrm{~nm}$ cannot ionize 1,2-dichlorobenzene, this feature does not appear in the one-color REMPI spectrum. As shown in Figure 36, the threshold for the onecolor, two-photon process is approximately $273 \mathrm{~nm}$.

Some effort was also expended to improve the optics for performing two-color REMPI experiments. In particular, the original proof of concept two-color experiments used a tunable uv photon in the $273 \mathrm{~nm}$ range combined with the fixed, $4^{\text {th }}$ harmonic of the Nd:YAG at $266 \mathrm{~nm}$. Although we will initially retain this overall configuration, the new optics will improve both the

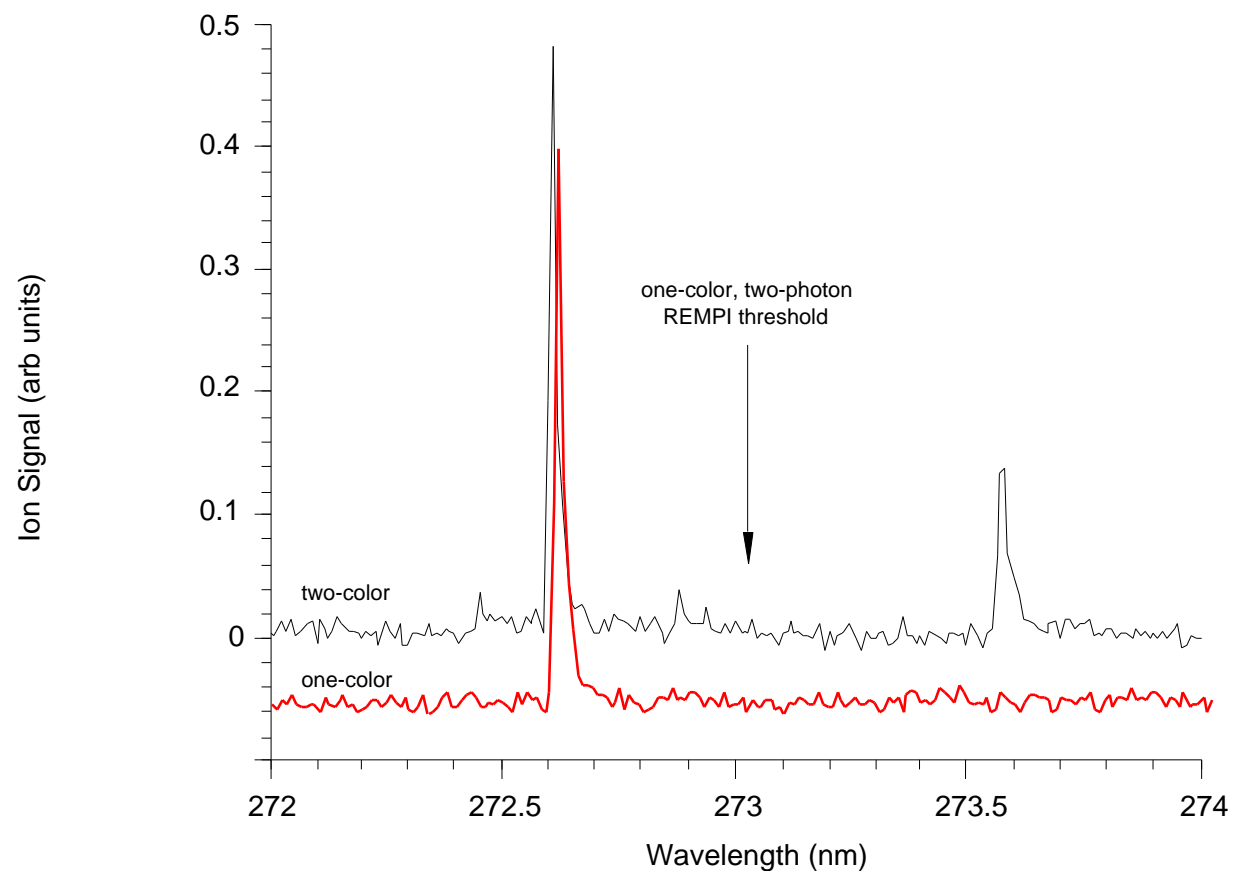

Figure 36. Wavelength dependence of the ion signal at $\mathrm{m} / \mathrm{z} 146$ for the jet-REMPI detection of 1,2dichlorobenzene. The lower trace was recorded using a one-color, two-photon, REMPI scheme. The upper trace was recorded using a two-color, two-photon REMPI scheme where the second color was at a fixed wavelength of $266 \mathrm{~nm}$. 
ease of performing two-color experiments and the sensitivity of the method resulting form improved stability and overlap of the tow laser beams within the ionization volume. We are also considering implementation of a $5^{\text {th }}$ harmonic generator to yield a fixed, $213 \mathrm{~nm}$ beam. The increased energy of the second photon will make possible the detection of wider range of compounds than is possible using the $266 \mathrm{~nm}$ color.

The REMPI absorption spectrum of 2-monochlorodibenzofuran (2-MCF) signal recorded for the parent $\left({ }^{35} \mathrm{Cl}\right)$ ion at $\mathrm{m} / \mathrm{z} 202$ as a function of the wavelength for a one-color, two-photon ionization process showed numerous, well-resolved, absorption features (Figure 35). We noted, however, that both the relatively low signal level and the lack of an apparent $S_{0} \rightarrow S_{1}$ band origin at long wavelengths might indicate that a two-color scheme would yield more sensitivity. We made a very preliminary test of this by combining a tunable photon with a fixed $266 \mathrm{~nm}$ photon, and indeed we see an enhanced signal.

We extended these two-color measurements which have verified and quantified the improvement. Figure 37 shows the measured REMPI signal for the parent ion (m/z 202) using different excitation/ionization schemes. Initially, only the fixed wavelength at $266 \mathrm{~nm}$ was used. With a energy of $0.2 \mathrm{~mJ}$, no REMPI signal was measured, as expected. After several minutes, $0.5 \mathrm{~mJ}$ of tunable light at $300.41 \mathrm{~nm}$ was added, resulting in an immediate increase in the signal. This signal remained constant for many minutes until the $266 \mathrm{~nm}$ fixed wavelength laser was blocked. Although the tunable laser at 300.41 remained on resonance, the REMPI signal deceased by a factor of ten. Finally, the OPO was detuned from resonance where $0.5 \mathrm{~mJ}$ at 300.3 $\mathrm{nm}$ again produced no measurable signal.

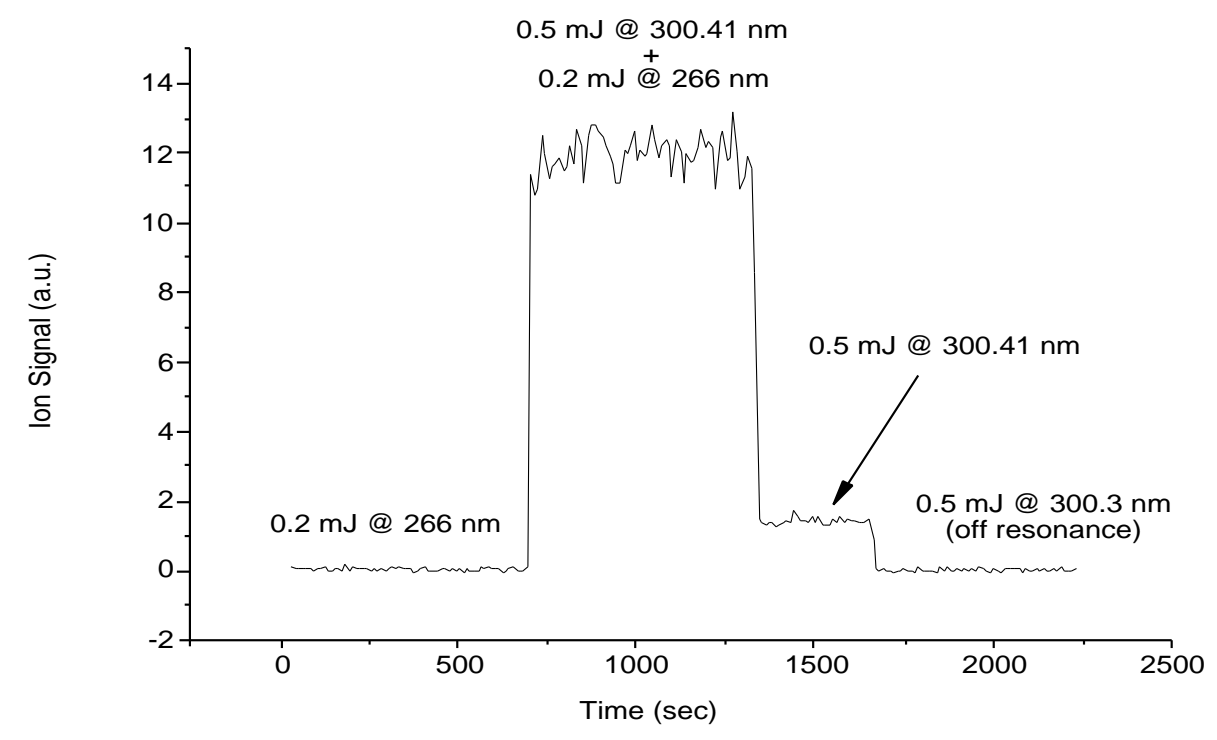

Figure 37. Dependence of the REMPI ionization signal for 2-monochlorodibenzofuran using different excitation schemes. 
It should be noted that the relatively large fluctuations in the two-color signal were due to variations in the energy of the $266 \mathrm{~nm}$ light. These variations were in turn caused by the lack of temperature stability of the externally mounted doubling crystal. A thermostated enclosure was added to the crystal, and these fluctuations have been greatly reduced by maintaining it at $35 \operatorname{deg}$ C.

\subsubsection{Calibration Methods}

Before jet-REMPI can be used as a CEM, a variety of field calibration methods must be developed. Three types of instrumental calibration is needed, one for each of the three key parameters; intensity, mass, and wavelength. Intensity calibration will require development of a standardized vapor delivery system capable of introducing a know, reproducible, concentration of a calibrant into the inlet.

Calibration of the mass and wavelength can actually be accomplished simultaneously through the appropriate choice of a target molecule. As an example of how this might be accomplished, we have chosen perdeuterobenzene $\left(\mathrm{C}_{6} \mathrm{D}_{6}\right)$ as a test molecule. While benzene and deuterobenzene $\left(\mathrm{C}_{6} \mathrm{H}_{5} \mathrm{D}\right)$ will be present in most incinerator sample streams, perdeuterobenzene will never occur naturally. Moreover, there are no commonly occurring interferents at mass 84 . Figure 38 shows the REMPI excitation spectrum for a 1:1 mixture of benzene and perdeuterobenzene. Note that over this narrow wavelength scan, each molecule exhibits only two, narrow, absorption features.

The perdeuterobenzene features are the same magnitude as the undeuterated molecule, and each is shifted by a fixed amount. Because the perdeuterobenzene features are so narrow, they can readily be used to check the wavelength calibration of the OPO. At the same time, a narrow wavelength can will provide two independent mass peaks (78 and $84 \mathrm{amu}$ ) that can be used to verify the time-to-mass conversion factors. In principle, it is better to have two or mass peaks with a larger mass separation, however, for a periodic check of the mass calibration, this approach may be adequate. Of course, other, higher molecular weight compounds can be added to the calibration mixture to accomplish this. 


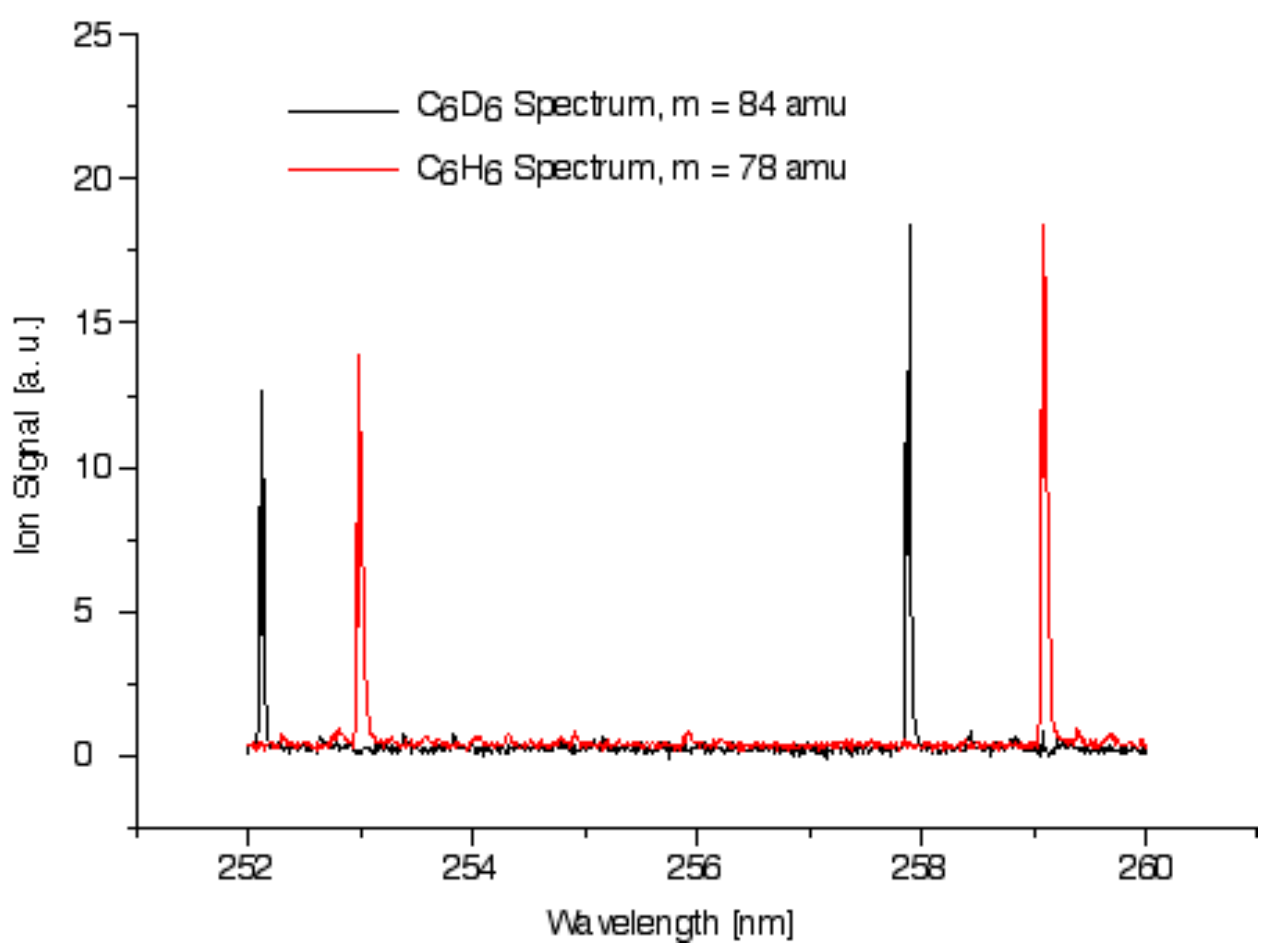

Figure 38. REMPI excitation spectra for a 1:1 mixture of benzene (78 amu) and perdeuterobenzene (84 amu) recorded simultaneously as a function of REMPI excitation wavelength.

\subsubsection{Off-line Sampling}

An important aspect of the dioxin CEM development effort is the sampling. While development of a sampling system was originally part of our planned effort, a number of factors have led us to reduce the level of effort devoted to this aspect of the work. Among the factors is the continuing uncertainty of the role of particulates in dioxin monitoring, the ongoing sampler/preconcentrator development efforts in Dr. Brian Gullett's laboratory at EPA, and the corresponding sampler work being pursed at MSE and DIAL.

As part of SRI's ongoing EPA effort to apply jet-REMPI to characterization of urban air pollutants, it was essential that an external sampling method be developed so that preliminary laboratory studies of the typical composition and concentrations of HAPs in urban air could be conducted prior to planning any field studies. Although that effort was not directly associated with the dioxin CEM project, the experience and results are sufficiently relevant that they are included here.

As a first approach, we used standard charcoal sorbent tubes connected to portable air metering pumps. By operating the pumps at a calibrated air flow for selected periods of time, we could sample known volumes of air. The sorbent tubes were then returned to the laboratory and the adsorbed organic compounds recovered using conventional solvent extraction methods. Because we needed to perform a chemical survey before applying jet-REMPI, we analyzed the extracted samples using GC-MS. Once a set of target compounds had been identified by GC- 
MS, the same sample was examined using jet-REMPI to confirm their presence and ascertain the signal levels available from our instrument.

The sampling method can be summarized as follows:

(1) Open hermetically sealed carbon filter element (CFE) and insert in sample pump inlet tube;

(2) Calibrate flow using a Buck flow meter

(3) Start sample pump and mark time

(4) At end of desired time duration, stop pump

(5) Remove CFE from pump inlet hose and cap open ends of CFE

(6) If at remote site, place capped CFE in cooler with icepack

The laboratory extraction procedure can be summarized as follows:

(1) Break open main sample volume of CFE.

(2) Pour filter carbon into sample vial

(3) Cap sample vial and label

(4) Repeat for breakthrough volume of CFE

(5) Add $1.0 \mathrm{ml}$ of $\mathrm{CS}_{2}$ to sample vial

(6) Wait minimum of $30 \mathrm{~min}$

The GC/MS analysis procedure can be summarized as follows:

(1) At beginning of day, run GC-MS calibration

(2) Run previously developed method "CS2MIX"

(3) Inject 2.0 $\mu 1$ of sample into GC/MS inlet

(4) After GC/MS run, analyze spectrum with NIST MS search

(5) At end of day, run bake column

The Jet-REMPI analysis procedure can be summarized as follows:

(1) Warm-up laser system

(2) Calibrate system, see note below

(3) Evacuate heated inlet sample flask

(4) Inject $10 \mu \mathrm{l}$ of sample into flask

(5) Select wavelength and mass

(6) Adjust gain

(7) Select filename and set beginning and ending wavelengths

(8) Plot and examine final REMPI data

Note that we created a calibration mix to check and adjust the wavelength ranges and mass calibrations. This was used in the beginning to set up the data acquisition software and occasionally to verify that the setup was still in spec. 
To test this sampling and analysis approach, we took samples were taken from open containers, gasoline tank headspace, automobile exhaust, and ambient air. In ambient air, we collected samples on a CFE for 3.5 hours, to obtain enough sample to use GC/MS as a survey tool. Only toluene shows in the GC/MS scan as a small signal. To use jet-REMPI, we diluted the sample by a factor of 20,000 to avoid overloading the instrument. This dilution is equivalent to a direct measurement with a sampling time of about $1 \mathrm{sec}$. All of the BTEX (benzene, toluene, ethyl benzene, and the xylenes) compounds were readily seen in the jet-REMPI instrument, and the three isomers of xylene can be individually quantified. Our preliminary study showed that not only can many urban air pollutants, such as BETX, can be detected quantitatively using jet-REMPI using long-term sorbent sampling, but more importantly, because of the high sensitivity of REMPI, these same compounds could also be detected in the field in near real-time without sampling and preconcentration.

\subsubsection{Improved Dynamic Range}

One of the critical limitations in our system is the 8-bit digitizer. At the present time, all digitizers operating above $200 \mathrm{MHz}$ are limited to 8-bits. This effectively yields a dynamic range of at most 255:1, but more realistically, about 100:1 due to random noise fluctuations. In practical terms, this means that once the parameters of the signal chain are fixed (MCP voltage, preamplifier gain, digitizer input attenuation) to keep the largest signals on scale, then any minor peaks less than $1 \%$ of full scale are not easily recovered. Signal averaging can help recover small peaks from the noise, but since the $S: N$ improvement varies only with $\sqrt{ }$ (repetitions), considerable additional time is required to achieve a substantial improvement. Another approach to improving the dynamic range is to employ a logarithmic amplifier (log amp). This approach has traditionally been problematic due to the limited speed of log amps and their inherent electrical noise. Newer log amps offer the possibility that this approach may be viable, and we are planning to test an Analog Modules Model 384 in the near future. One other option to improve the dynamic range of the digitizer is to use two digitizers in tandem, each with a different gain. The resultant signal is then reconstructed from the two separate digital traces using the relative gains. The major disadvantage of this approach is the $\$ 6500$ cost for each digitizer.

Because we have a spare Signatec DA500A digitizer board, we investigated the use of a second digitizer as a means of increasing the effective dynamic range of our data acquisition system. The DA500A digitizers has the capability of being configured in a master-slave arrangement. This configuration uses only a single channel counter to advance each digitizer, ensuring perfect temporal registration of the two traces. Test software was written to implement the master-slave configuration and a $40 \mathrm{MHz}$ arbitrary waveform generator was used to provide a test signal whose relative peak amplitudes could be easily controlled. 
Figure 39 shows the results of the test. The first digitizer was adjusted to have a full scale input of $0.237 \mathrm{Vdc}$, while the second digitizer was set for a full scale of $1.282 \mathrm{Vdc}$. Both digital traces (shown as the upper and middle graphs, respectively) were transferred to the computer after each trigger and an effective signal synthesized (lower graph). The algorithm used to synthesize the resultant signal was simply:

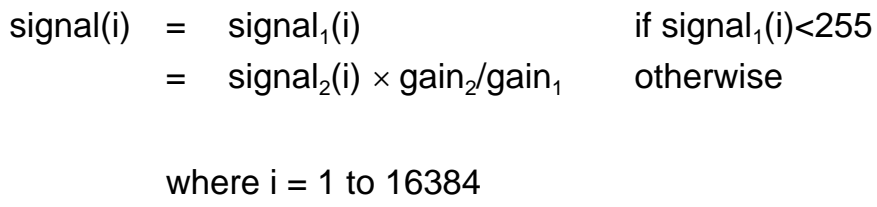

For the data shown in Figure 39, the gain ratio was 5.409, yielding an effective dynamic range of 10-bits, or nearly one order of magnitude better than a single digitizer. Note that in the upper trace, the signal periodically exceeds full scale of the first digitizer, while the gain of the second digitizer is such that the middle trace is always less than full scale. Although not apparent, all of the low level signal details visible in the upper trace are reproduced exactly in the bottom trace.

In principle, a gain ratio of more than 18:1 can be achieved using the DA500As which would provide an effective dynamic range of 12 bits. Currently, however, the output of our fast preamplifier is limited to less than 2 volts, so the full 12 bits cannot be realized. Nonetheless, the results shown in Figure 39 show that an order of magnitude improvement can be achieved with minimal additional programming effort. 


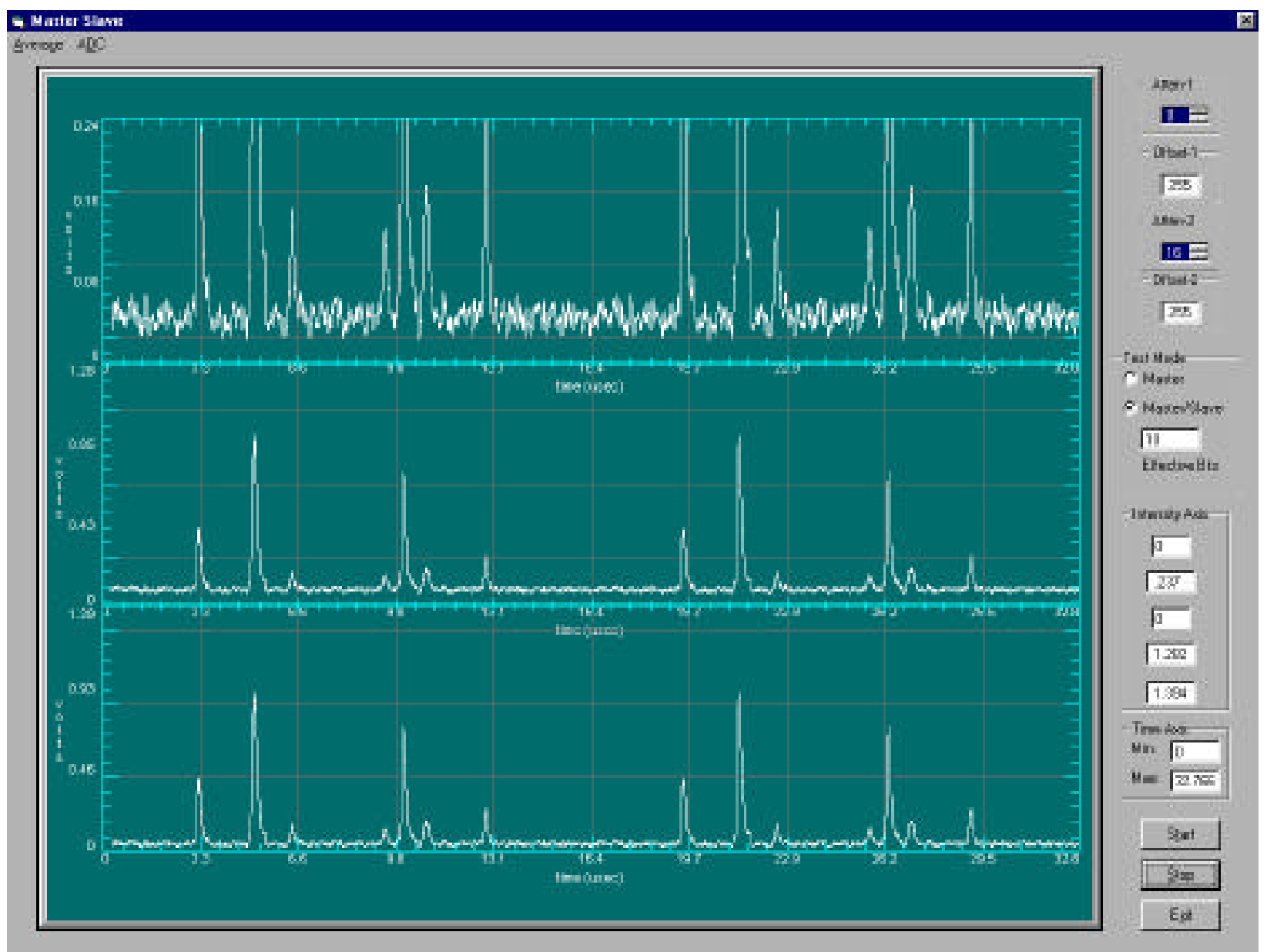

Figure 39. Test of dual digitizer data acquisition system. The upper trace was acquired on the first digitizer with a full scale input of $0.237 \mathrm{Vdc}$. The center trace was acquired on the second digitizer with a full scale input of $1.282 \mathrm{Vdc}$, and the lower trace is the composite signal produced according to the algorithm described in the text.

\subsubsection{Field Work EPA NRMRL}

SRI had a unique opportunity to perform a series of pseudo-field measurements without the time and expense associated with transporting the instrument to a field site. This was possible because by using the REMPI apparatus that SRI built for Dr. Brian Gullett at the EPA's NRMRL. Since that apparatus is essentially a duplicate of the one currently in use at SRI, measurements taken with the system in conjunction with EPA's combustion facility allowed us to make these "field-like" measurements.

Dr. Oser, therefore, traveled to NRMRL and was able to perform numerous experiments using the SRI-built REMPI apparatus in conjunction with the EFR flow reactor. A diagram of the flow reactor is shown in Figure 40. An outline of these experiments was provided in 


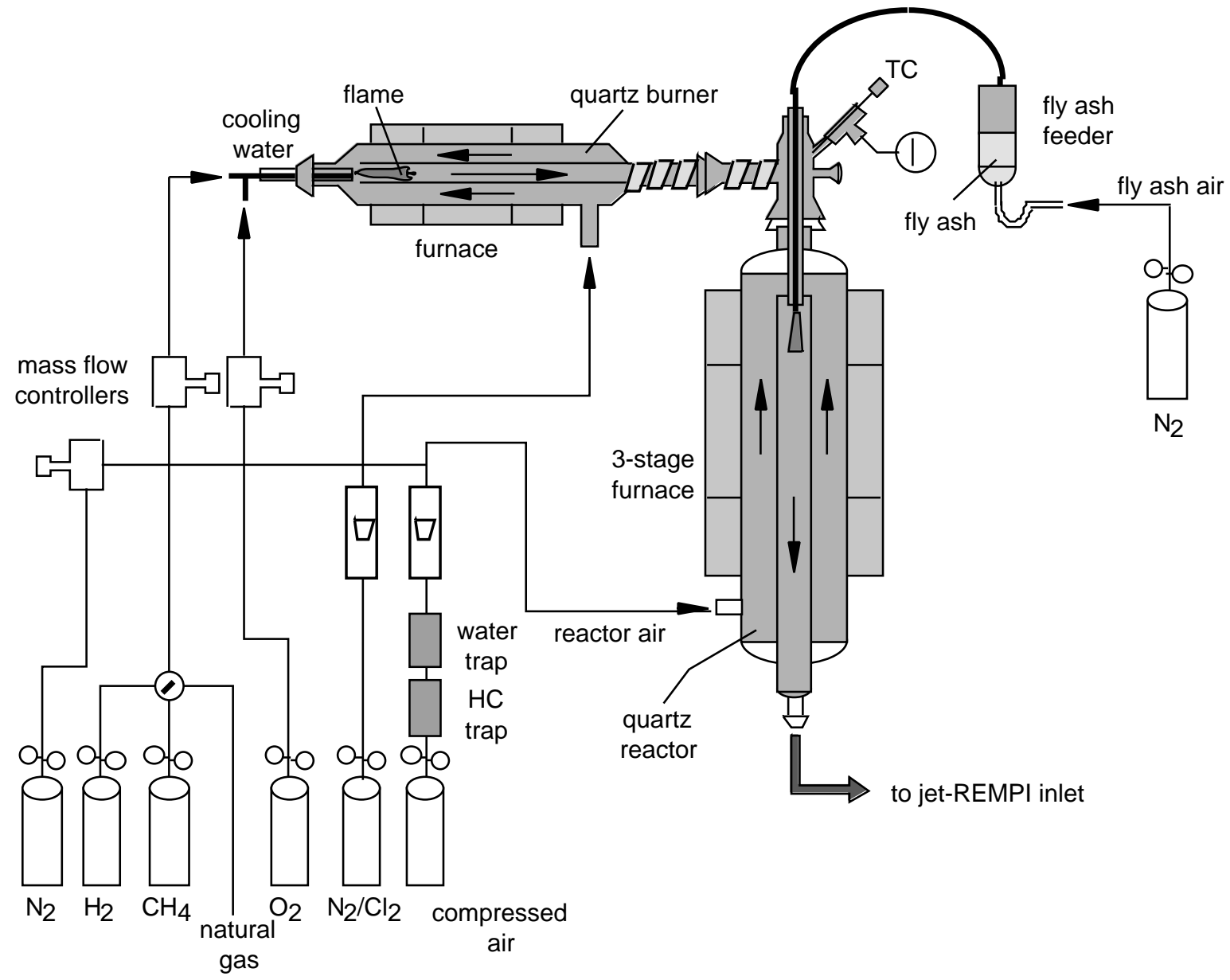

Figure 40. Diagram of the US EPA combusiton research flow reactor used in the field measurements.

Appendix A Proposed Test Plan, which was submitted prior to beginning the work. The outcome of these experiments is being critically examined and will be the focus of a joint SRIEPA publication. It should be noted, however, that for the most part the experiments were successful, and should yield considerable new information. It is also worth noting that the apparatus was operating at least as well as when it was first installed at RTP, with typical detection limits in the low ten's of ppt for simple aromatics such as benzene. The preliminary results are reported here.

Among the most intriguing observations was the detection of several interesting species that were identified in a nominally "clean" methane flame. Benzene and phenol were both positively detected in the off-gas stream of the reactor. In addition, clear evidence was also found for aniline produced in the EPA reactor under the test conditions. For all species, the measured spectra are essentially identical to those recorded using a "clean" test gas mixture and all were easily detected in the exhaust stream. Furthermore, their presence could be entirely 
attributed to the methane combustion chemistry as the background levels were not detectable in the absence of the flame.

Figure 41 shows the time dependence of the phenol signal at m/z 94 with the laser tuned to the primary resonance at $274.1 \mathrm{~nm}$. The time resolution of this data was 1 second, corresponding to 10 laser shots per data point. Between $t=0$ and 250 seconds, the background signal was measured with no reactant gases flowing. At 250 seconds, the methane and oxygen reactants were introduced but without igniting the flame. Note that prior to flame ignition, the phenol signal was essentially absent. At the point indicated on the figure, the flame was ignited, and the phenol signal rose rapidly and remained more or less constant until the flame was extinguished. With the flame off, the phenol signal initially decreased rapidly followed by a slower decrease back to the baseline. The slower decay was likely due to residual phenol in the sampling line and pulsed valve. Increasing the temperature of these components would likely decrease the phenol clearance time. Under the REMPI conditions used to measure the data in Figure 41, we estimate the phenol concentration to be $150 \mathrm{ppt}$.

Figure 42 shows a similar time history recorded for the aniline signal at $\mathrm{m} / \mathrm{z} 93$ and a wavelength of $292.8 \mathrm{~nm}$ (see Figure 43). In this measurement, the methane flame equivalence ratio was also 0.8. Again, the aniline signal was absent until the flame was ignited, at which point it rose rapidly and remained easily measurable until the flame was extinguished. No estimate has been made yet for the concentration of aniline in the exhaust gas stream.

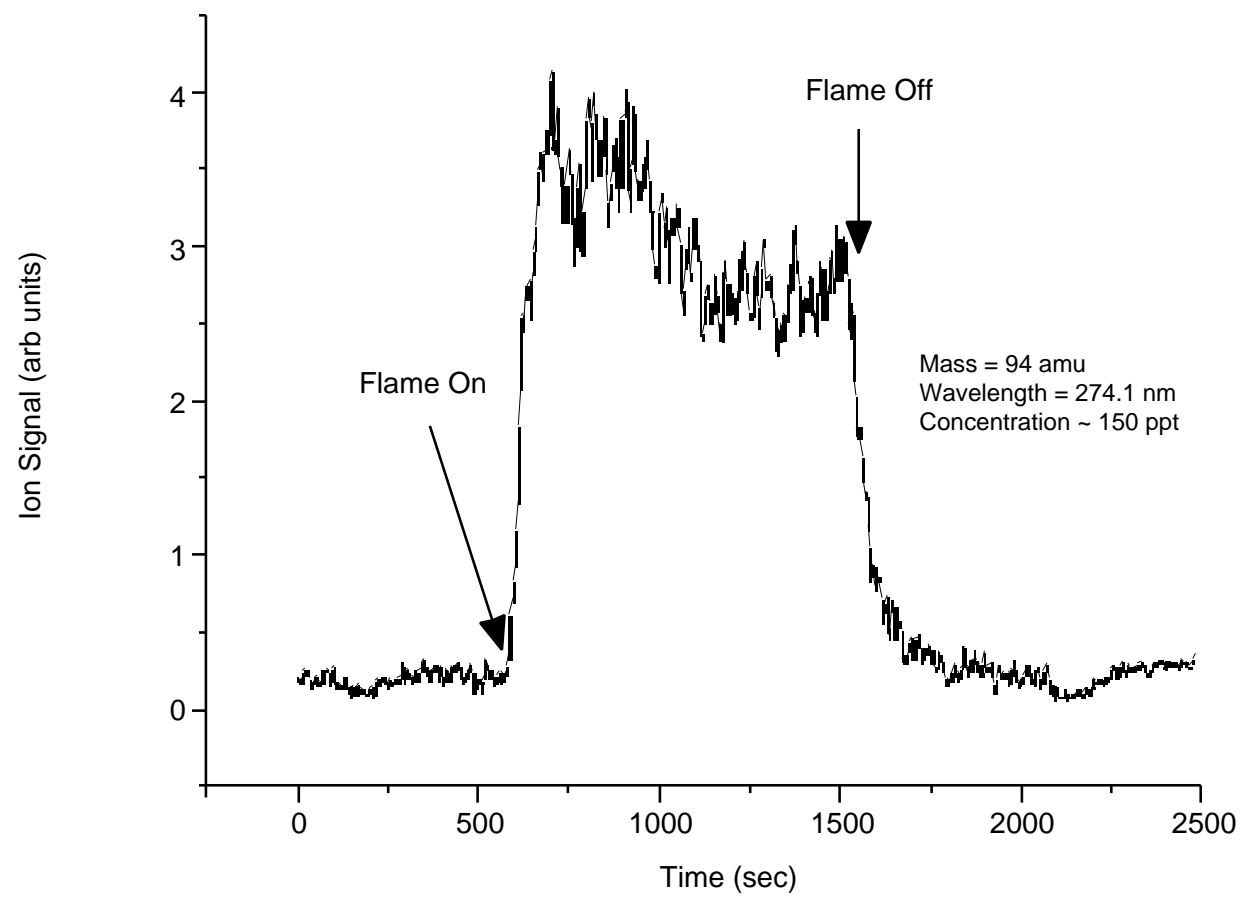

Figure 41. Time dependence of phenol measured in a lean methane flame at EPA. 


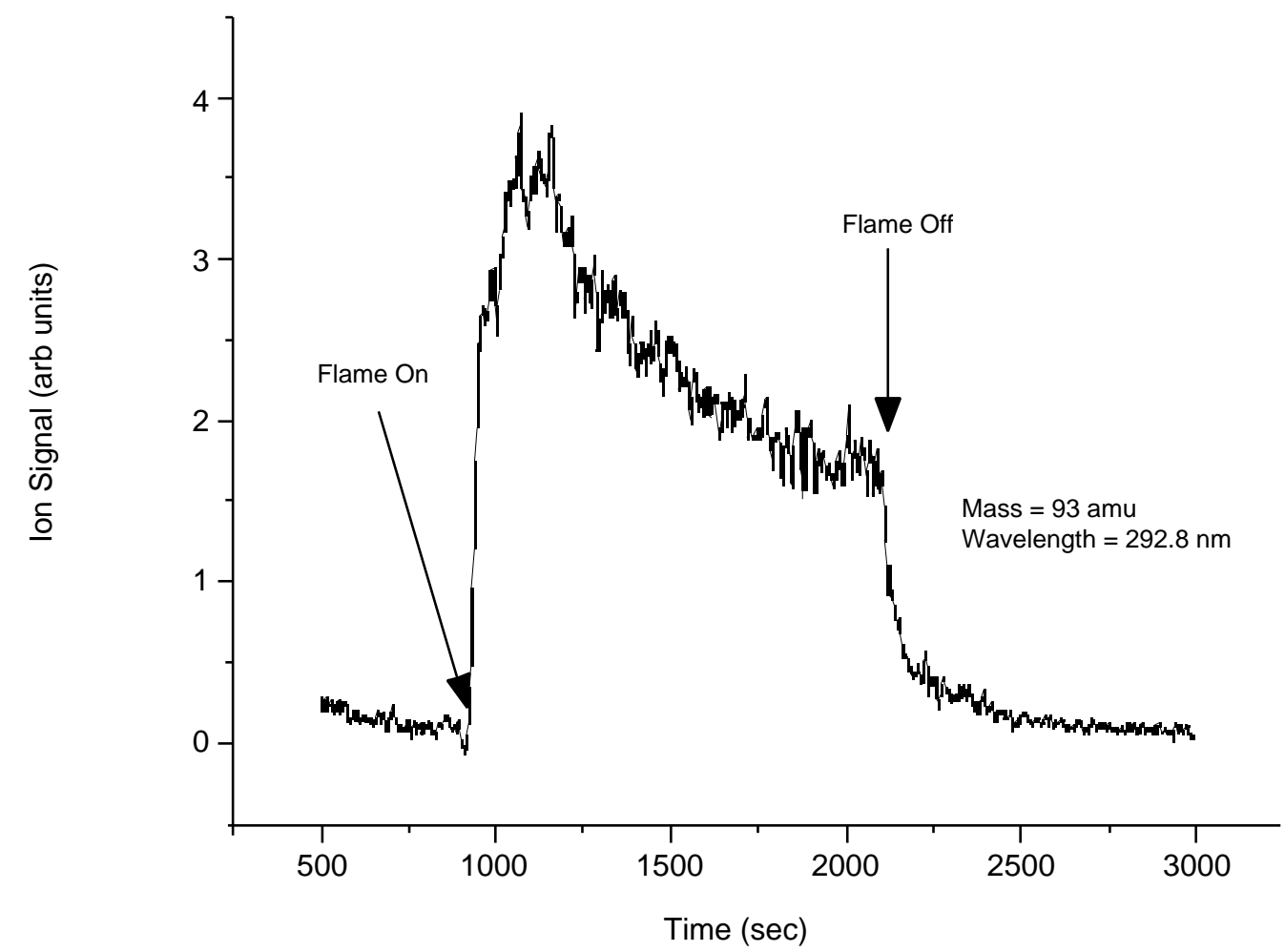

Figure 42. Time dependence of aniline measured in a lean methane flame at EPA.

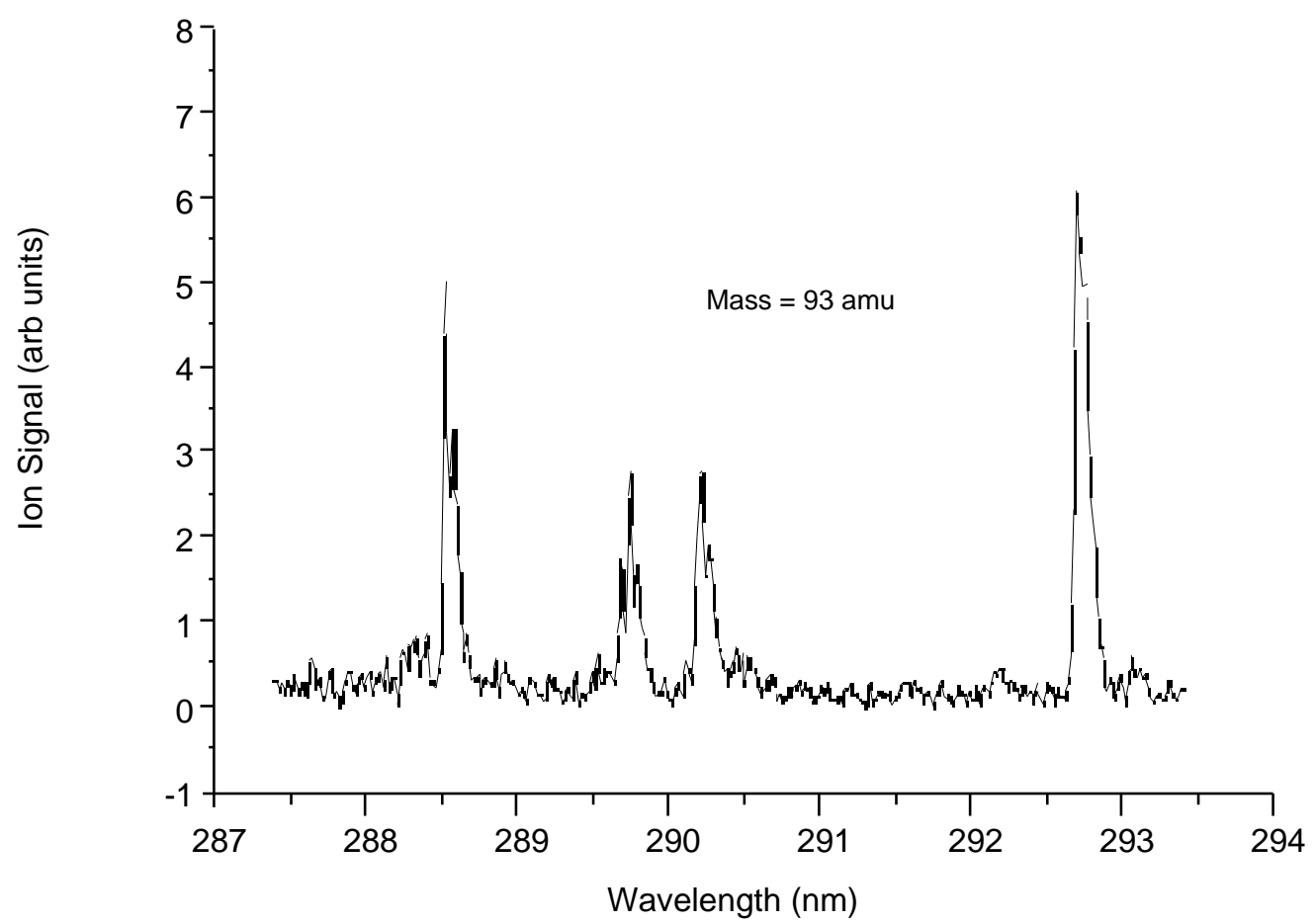

Figure 43. Wavelength dependence of the aniline jet-REMPI signal measured in a lean methane flame at EPA. 
Because the observation of aniline in the exhaust stream from a nominally pure methane flame was unexpected, a wavelength scan was recorded at $\mathrm{m} / \mathrm{z} 93$ to confirm the identification. Figure 43 shows the measured REMPI signal over the wavelength range from $287.5 \mathrm{~nm}$ to 293.5 $\mathrm{nm}$. This spectrum corresponds to the known REMPI absorptions for aniline under jet-cooled conditions. Note that the data in Figure 42 was measured with the laser tuned to the primary resonance line at $292.8 \mathrm{~nm}$.

\subsubsection{Other Collaborations}

During the course of this effort, SRI interacted with a number of other research groups involved in closely related efforts. Drs. Crosley and Oser visited the DIAL group at Mississippi State University to discuss complimentary field experiments that could be performed at their facility as part of our future efforts to develop and test jet-REMPI under a variety of conditions.

In light of SRI's plans to eventually test the jet-REMPI system on a pilot-scale combustor, it became clear that we would need to coordinate our instrument design efforts with those of the sampler developers. As a result, a visit was made by Drs. Coggiola and Oser to the facilities of MSE during this reporting period. Detailed technical discussions were held covering their sampler probe design, function, and possible integration with our system. Plans were also discussed for possible field experiments to be conducted at MSE that would be designed to make a side-by-side comparison of the real-time jet-REMPI detection of important organic compounds with MSE's sorbent tube collection and offline GC-MS analysis.

SRI has recently been asked to participate in an international effort to assemble a REMPI data base of common chemicals detectable by this method. The consortium has applied to IUPAC for recognition and funding. SRI intends to share with the group our library of REMPI spectra, and furthermore, we will use this opportunity to help develop a uniform reporting format and searchable database.

In addition to these collaborations, SRI maintains a close working relationship with Dr. Gullett.

\subsection{DIOXIN CEM DESIGN SPECIFICATION}

\subsubsection{TEQ Monitor}

Based on the results obtained to date, it is not possible to conclude that an instrument based on a jet-cooled molecular beam combined with REMPI and TOFMS will ultimately provide the sensitivity, selectivity, and time response required for a dioxin CEM. This observation is based on a number of factors, including the "chlorine effect", and the unresolved 
issues associated with the role of particulates in the formation and transport of dioxins and furans.

The so-called chlorine effect has been observed and verified by every group applying REMPI to polychlorinated dioxins. This effect, which manifests itself as a significant reduction in ionization efficiency for each additional chlorine added to a congener, may substantially limit the ability of REMPI to achieve the sub-ppt detection levels required of a compliance CEM. While the chlorine effect has not been verified for all important congeners, and hence may be less important than currently believed, there is sufficient evidence to make it reasonable to assume that it will impact the detectability of more highly chlorinated congeners. There are a variety of potential ameliorating techniques that might be employed. For example, the use of two-color REMPI, shorter laser pulses, and improvements in the ion extraction and detection could overcome, at least in part, the reduced ionization efficiency of highly chlorinated dioxins.

The issues associated with sampling in general, and the role of particulates in particular, has not been adequately addressed by the dioxin CEM community to the point where useful guidance is available. One of the most significant technical issues in regard to sampling is the requirement to maintain all surfaces of the sampling line at an elevated temperature such that the most highly chlorinated dioxin and furan congeners of interest $(2,3,7,8$-chlorine substituted species) are not lost due to condensation. This thermal requirement extends to the pulsed valve, as well. Moreover, the actual surface materials that are in contact with the sampled vapor stream are critical, further exacerbating the difficulties. Finally, a key issue is the disposition of particulates in the sample stream. Because particles, and any adsorbed dioxin and furan congeners, are included in the standard method 23 sample collection and analysis train, they are a de facto part of the TEQ determination. Thus, since particulates contribute to the TEQ, they too must be included in the REMPI TEQ measurement. Despite their potential importance, there are currently no adequate sampling and desorption methods suitable for use with a real-time dioxin CEM regardless of the detection methodology. It seems clear that more work will be required in the future to address these issues.

As a general design specification, a jet-REMPI based dioxin CEM would consist of the basic components used in our laboratory prototype, albeit with significant improvements where feasible. The generic specification therefore consists of: (1) a heated sampling and transfer line whose surfaces are chemically inert toward all congeners of interest; (2) a heated, pulsed valve with similar thermal and chemical characteristics; (3) a narrow band, tunable, pulsed uv laser source operating at as high a repetition rate as the detection electronics, pulsed valve, and vacuum system will allow; (4) ion extraction optics capable of collecting and focusing the vast majority of nascent ions into the mass spectrometer; (5) a time-of-flight mass spectrometer with mass resolution exceeding 500, and preferably at least 1000; (6) an ion detector that is linear with incident current over 3-4 orders of magnitude without signal distortion or electronic ringing; 
(7) a preamplifier with a minimum $500 \mathrm{MHz}$ bandwith; (8) a high speed digitizer providing at least 12 bits of effective resolution and a single shot time resolution of 1-2 nsec and a high-speed data transfer rate to the data acquisition computer; (9) on-line wavelength, mass, and intensity calibration; and (10) a fully automated, autonomous control system.

There are numerous other operational specifications for many of these components that are not explicitly stated here. For example, the uv output of the laser system must be very stable in wavelength, linewidth, optical beam quality, and intensity independent of the thermal and mechanical environment in which it would operate. Moreover, the laser conditions must be reproducible over long periods of time to avoid the need for recalibration. While this might be accomplished with a combination of temperature control and vibration isolation, these are not necessarily simple engineering issues. Similarly, the optical alignment of the ionization laser beam with the ion source region is critical to maintain optimum overlap between the laser and pulsed gas jet. Variations in the spatial overlap between these two can lead to significant degradation in both the signal level and the apparent mass resolution. Thus, mechanical rigidity of the entire system is a critical engineering design parameter.

It is instructive to review the current state of development of each of the key components outlined above. Table 3 summarizes the requirements and the currently available or developmental capabilities for each component.

\section{Table 3}

COMPARISON BETWEEN REQUIRED AND CURRENTLY AVAILABLE PERFORMANCE SPECIFICATIONS FOR KEY COMPONENTS OF A JET-REMPI SYSTEM

Component

1. heated sampling and transfer line

2. heated, pulsed valve

3. narrow band, tunable, pulsed uv laser source

4. ion extraction optics

5. time-of-flight mass operate at as high a repetition rate as the detection electronics, pulsed valve, and vacuum system will allow

capable of collecting and focusing the vast majority of nascent ions into the mass spectrometer

mass resolution exceeding 500 ,
Curently Available

could be assembled using existing technology with the exception of particulate treatment

problematic, both with regard to chemical inertness of the surfaces and thermal performance

could be assembled using existing, commercial systems

highly refined, and meet the demands

can be readily assembled or 
spectrometer

6. ion detector

7. preamplifier

8. high speed digitizer

9. calibration

10. control system and preferably at least 1000

linear with incident current over 3-4 orders of magnitude without signal distortion or electronic ringing

minimum $500 \mathrm{MHz}$ bandwith

at least 12 bits of effective resolution and a single shot time resolution of 1-2 nsec and a highspeed data transfer rate to the data acquisition computer

on-line wavelength, mass, and intensity

fully automated, autonomous purchased

can be readily assembled or purchased

can be readily assembled or purchased

could be assembled form commercially available systems, high cost

could be developed using existing technologies

could be developed using existing technologies

\subsubsection{Alternative REMPI-Based Instrumentation Schemes}

While a true TEQ compliance monitor is not yet possible using the jet-REMPI approach, the technique may prove useful as a surrogate, or indicator monitor. This application would involve continuous measurement of surrogate compounds, such as lowly chlorinated dioxins and furans, or other species whose concentrations have been previously correlated with the TEQ. Such an instrument may not require the extreme sensitivity of a compliance monitor although the high degree of chemical selectivity could remain important. Specific operational criteria depend strongly on the specific target compounds.

The configuration of such an instrument would depend strongly on the specific chemical surrogate(s) chosen and their anticipated concentration(s) and form. Assuming that the surrogates were lowly chlorinated dioxin and furan congeners with three of fewer chlorine atoms, then the laboratory prototype system developed and used in this effort would form the basic design specification. In particular, the pulsed jet inlet, narrow-band laser, and modest resolution TOFMS would all be required to maintain adequate sensitivity and selectivity. Because only lowly chlorinated congeners would be of interest, the thermal issues associated with both the transfer line and the pulsed valve would be easily mitigated using conventional technologies.

Should a robust correlation between simpler surrogates, such as chlorobenzenes, and the true TEQ be established for a particular combustion system, then a REMPI-based instrument might be simplified as well. These simplifications might be include replacement of the pulsed 
valve with a capillary inlet, and the use of a wider band, tunable uv laser source, or even, a fixed frequency $(266 \mathrm{~nm})$ pulsed source. The capillary inlet would consist of a length of uncoated, glass capillary tubing that forms a gas flow restrictor, limiting the pressure in the ionization chamber to a reasonable value. Such an inlet is continuous, rather than pulsed, thus the average flow must be considerably less than the currently used pulsed flow. Nonetheless, the pulsed ionization source would still allow use of TOFMS detection.

The benefits of this simplified design include considerably lower cost, reduced size and complexity, and improved robustness and easy of use, particularly if a fixed frequency laser source is used in combination with a pulsed valve. The drawbacks include reduced sensitivity and selectivity, the need for an acceptable surrogate-TEQ correlation that is valid over a range of operating conditions and feedstocks, and the possibility that the resulting measurements would not satisfy regulatory requirements. In fact, the measurements made using such a system are best considered as a real-time indication of the process conditions, rather than as a real-time emissions monitor. Such a measurement could be very useful in ensuring that the combustion process is operating within acceptable design parameters, and by inference, in compliance with emissions limits.

It remains to be shown that appropriate surrogates are available whose levels correlate with the TEQ. If acceptable surrogates are found, then it must be demonstrated that they can be measured in real-time and at the expected levels with a simplified instrument.

\section{CONCLUSIONS}

We can conclude from our work to date and the extremely demanding regulatory compliance monitoring requirements involving both congener specificity and sub-part-per-trillion sensitivity with near real-time speed, that it is not as yet possible to specify a system configuration for a true dioxin compliance monitor. As detailed in this report, this conclusion is based on a number of factors, including both scientific, technical, and engineering issues.

We have developed a number of concepts for instrumental improvements that will substantially increase our sensitivity while maintaining the exceptional selectivity required of a dioxin CEM. In addition, we have developed several system configurations with varying degrees of functionality that can be further developed and deployed for process monitoring, surrogate measurements, and potentially, as a dioxin control CEM.

\section{FUTURE WORK}


The direction of future work on the development and application of the jet-REMPI method for continuous emissions monitoring will depend strongly on the intended application. Should a true TEQ compliance monitor be required, then the effort would clearly focus on improving the sensitivity to overcome the apparent chlorine effect. At the same time, the critical sampling issues, especially those associated with particulates, must be addressed and satisfactorily resolved.

While SRI has measured the jet-REMPI spectra for many pollutants of interest, including selected polychlorinated dioxins, many additional congeners must be similarly investigated so as to expand the library of detectable compounds. All work to date indicates that this readily done for dioxins and furans with three or fewer chlorines using the current system. For more highly chlorinated species, the two-color approach will be require, although we have demonstrated during this effort that it works well and is easily implemented in the laboratory. Inclusion of the more highly chlorinated species in the spectral library would also require a high temperature inlet and pulsed valve, both of which are developmental items. Sensitivity is not an issue in regards to acquiring REMPI excitation spectra, so no additional developments would be required for that work.

As detailed elsewhere in this report, sampling issues remain largely unresolved. The use of fast preconcentration/desorption may be a viable means of improving the effective sensitivity of the method. If the preconcentrator can introduce some degree of preseparation, then it could improve selectivity as well. Sample preconcentration might also help to resolve the particulate problem by allowing collection and then thermal desorption of adsorbed species from particles in the exhaust stream. Clearly, this process will be difficult to implement in a quantitative way while maintaining the time response of the system.

\section{REFERENCES}

1. K. Ballschmiter, Bacher, Dioxine, VCH, Weinheim, Germany (1996).

2. F. Chanania, EPA Office of Solid Waste, personal communication (February 1997).

3. H. Oser, R. Thanner, H. H. Grotheer, B. Gullett, N. Bergan French, D. Natschke, "DLR JetREMPI as a Continuous Emissions Monitor: Measurements of Chlorinated Dibenzodioxins," in Proceedings, 1997 International Conference on Incineration and Thermal Treatment Technologies, (May 1997).

4. Brian Gullett, EPA National Risk Management Laboratory, Combustion Research Branch, personal communication (April 1997). 
5. H. Oser, R. Thanner, and H. H. Grotheer, in Proceedings, 1996 International Conference on Incineration and Thermal Treatment Technologies (1996), pp. 387-392.

6. R. Zimmermann, U. Boesl, D. Lenoir, A. Kettrup, Th. L. Grebner, and H. J. Neusser, Int. J. Mass Spectr. Ion Phys. 145, 97 (1995).

7. C. Weickhardt, R. Zimmermann, U. Boesl, and E. W. Schalg, Rapid Comm. Mass Spectrom. 7, 183 (1993).

8. E. A. Rohlfing, 22nd Symp. (Int.) on Combustion, The Combustion Institute, p. 1843 (1988).

9. N. Bergan French, B. K. Gullett, H. Oser, H. H. Grotheer, D. Natschke, "Jet-REMPI Dioxin CEM Test Program Final Report,” DOE Westinghouse Savannah River (1997).

10. M. Rossi and D. J. Eckstrom, Chem. Phys. Lett. 120, 118 (1985).

11. M. Rossi and H. Helm, J. Chem. Phys. 87, 902 (1987).

12. E. Y. Xu, T. Tsuboi, R. Kachru, and H. Helm, Phys Rev. A36, 5645 (1987).

13. M. Okruss, R. Müller, and A. Hese, J. Mol. Spectrosc. 193, 293 (1999).

14. E. B. Wilson, Jr., J. Chem. Phys. 3, 276 (1935).

\section{ACKNOWLEDGEMENTS}

The authors wish to acknowledge the technical contributions of Mr. Steve E. Young, and Mr. William C. Olson, SRI, for their many contributions and helpful technical suggestions and discussions. In addition, we acknowledge the technical contributions of undergraduate students Ms. Qui Luu, Ms. Katherine Copic, Mr. Steven Schuh, and Mr. Gregory Grist who contributed to this effort as part of the Research Experience for Undergraduate program under sponsorship of the National Science Foundation.

Significant contributions were made by Mr. Bengt Volquadrdsen, a visiting SRI International Fellow.

We gratefully acknowledge the technical guidance and encouragement provided by Mr. Ronald K. Staubly, National Energy Technology Laboratory, Morgantown, WV, Mr. Stephen J. Priebe, Idaho National Engineering Laboratory, Dr. Stephan Weeks, Bechtel-Nevada, and Ms. Nina French, Sky+, Napa, CA. 
During the course of this effort, we benefited from numerous discussions and visits with Drs. Brian Gullett and Lukas Oudejans, US EPA, RTP, NC; the technical staff of MSE

Technology Applications, Inc., Butte, MT, in particular, Dr. Clarence Whitworth and Mr. Dan Battleson; as well as the technical staff of DIAL, Mississippi State University, Mississippi State, MS.

Selected portions of the work described in this report were jointly supported by this contract and EPA Agreement Number R 82792701-0, "Characterization of Urban Air Toxics Sources In Support of HAPs Emission Control Strategies". 


\section{APPENDIX A \\ Preliminary Test Plan \\ Field Measurements Using Jet-REMPI: Application To Flow Reactor Combustion Processes}

Research test plan for the visit of Dr. Harald Oser, June 18-29, 2001, to EPA/APPCD, Research Triangle Park, NC. This test plan is based on the assumption that as of June 18, 2001:

- The EFR flow reactor will be available for tests.

- A REMPI library including additional aromatic compounds will be available based on a recently purchased mixture of aromatics.

- A non-Teflon (quartz or stainless steel) sampling line is available.

The preliminary test plan includes three types of experiments list in order of priority:

1. Flue gas sampling versus $\mathrm{N}_{2}, \mathrm{~N}_{2} / \mathrm{H}_{2} \mathrm{O}$ environment. What is the effect of flue gas on the REMPI signal of a known compound, for example, deuterated benzene? What compounds can be identified as PICs?, versus equivalent ratio?

The jet-REMPI apparatus will be setup to sample directly from the EFR flow reactor. Initially, deuterated benzene will be injected into the inlet, and a baseline signal level established. The same species will then be monitored with the flow reactor in operation to assess the impact of flue gas on detection. Flow reactor conditions will be varied as time allows. A search will be made for products of incomplete combustion using the expanded REMPI aromatic library.

2. Sampling for dioxins using $\mathrm{N}_{2}$ carrier gas spiked with known levels of selected dioxin congeners (non- and lowly-chlorinated). Investigate possible production of dioxins in the flue gas resulting from addition of fly ash and/or reactive species.

Sampling issues. One sampling issue regards the possible influence of water on the REMPI signal. Prior work at EPA has shown the deuterated benzene signal increases when water vapor is present in the sample stream. These measurements were made using a Teflon transfer line, and the will be repeated using either a stainless steel or, preferably, quartz sampling probe. Issues regarding sample recovery, adsorption, pre-exposure of lines, and response times may be investigated if time permits. 


\section{APPENDIX B}

\section{SRI Proprietary Design Concepts for Improved Slit-Type Nozzle and Ion Extraction Optics}

Previous jet-REMPI studies of chlorinated aromatic compounds, show that achievable sensitivities are in the low parts-per-trillion range. Our preliminary experiments, described below, confirm this observation. While this represents excellent chemical sensitivity that is sufficient for many analytical applications, it must be improved by several orders of magnitude for a dioxin and furan CEM.

SRI developed several concepts for improving the sensitivity of our jet-REMPI instrument. One of the most promising improvements is the use of slit-type pulsed nozzle in place of the standard valve with a circular orifice. A slit-type pulsed valve will produce a wide gas jet that, when crossed by the laser, will yield a much larger ionization volume than the existing configuration. Although slit-type pulsed gas valves have been developed previously, they have not been used with laser ionization, and therefore, no designs exist for the critical ion extraction optics that can accommodate a line-source of ions.

Using SIMION, we have now developed a new ion optical design, shown in Figure B1. Note that the lenses are not circular, but rather slits with the longer dimension into the plane of the page. The dotted lines indicate open mesh grids used to maintain the proper electrostatic fields and to shield the ions from the distortion induced by the large, grounded, valve body approximately $1 \mathrm{~cm}$ from the laser beam. Note also that the vertical and horizontal scales are not equal in this sketch; the horizontal dimension is compressed to keep the full $30-\mathrm{cm}$ length visible, while the vertical dimension is expanded to show the electrode detail.

SIMION simulations of this source show excellent ion beam behavior in the field-free flight tube to the left of the sketch. This behavior can be seen by the ion trajectories shown in Figure B1, which shows the very tight ion focal point just inside the second lens element to the left of the laser ionization region. This will allow us to incorporate a small-diameter skimmer at that point and hence to provide differential pumping within the source region to reduce the gas load due to the much larger open area of the slit nozzle as compared with the conventional circular nozzle. Some of the key characteristics of the new source are listed in Table B1.

In addition to the SIMION simulations described above for the design of the ion extraction optics, we have begun performing gas dynamic simulations to better understand optimal slit configurations, the tradeoffs involved with the use of a slit nozzle instead of a circular nozzle, and any resulting constraints on our improved ion optics. These simulations are 
based on method of characteristics calculations for free jet expansions from circular and slit shaped nozzles. ${ }^{16-18}$ The accuracy of these calculations, which assume an isentropic gas

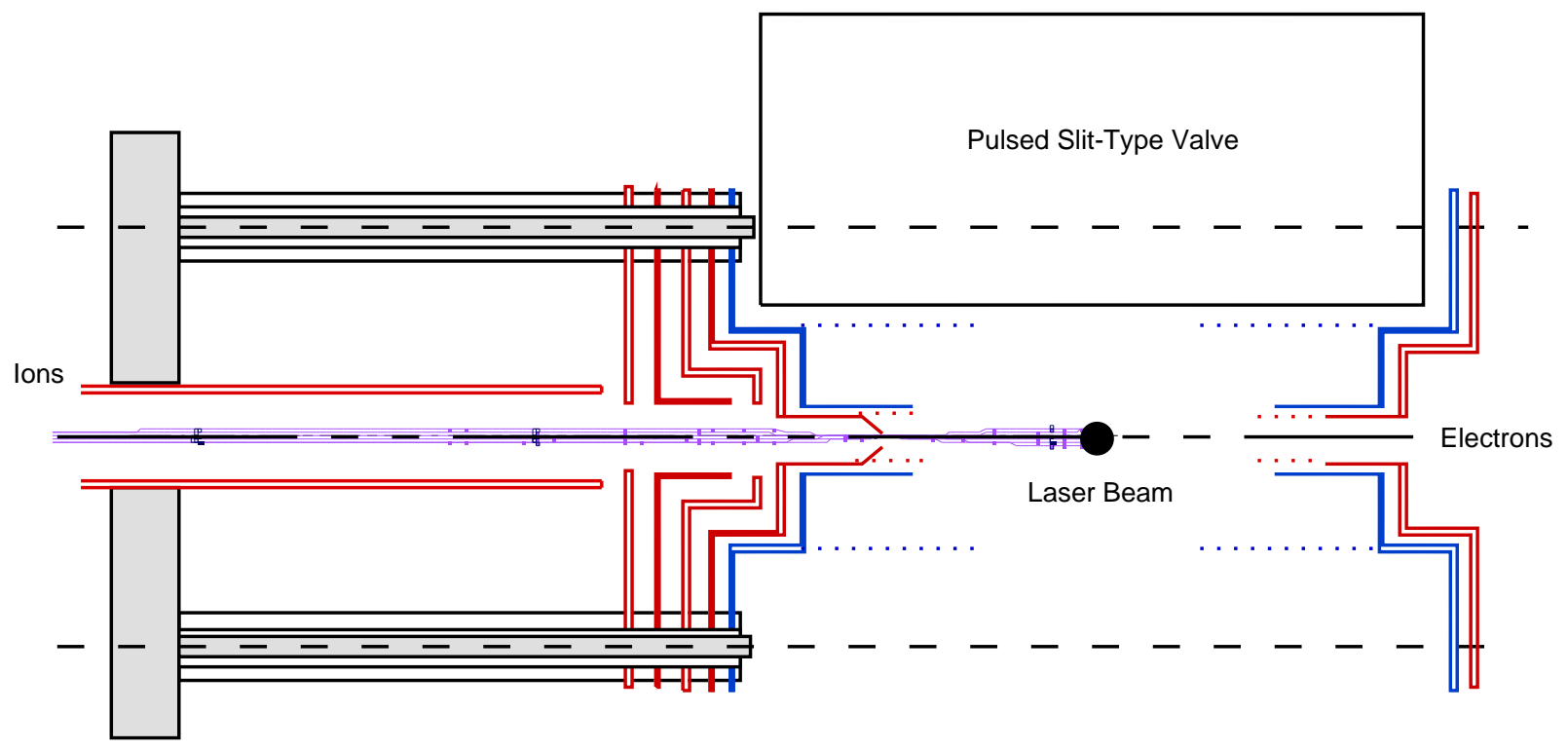

Figure 8. Design of the ion extraction optics for a slit-type pulsed valve. Ion trajectories were calculated by SIMION for realistic ionization and source conditions.

\section{Table B1 \\ CHARACTERISTICS OF THE SLIT-TYPE ION SOURCE}

Feature

Use of wire mesh grid structures

Symmetrical arrangement of acceleration and repeller electrodes

\section{Effect}

- No disturbance of electrostatic field distribution with small separation between laser beam and metal valve.

- Smallest possible distance from grid to laser beam of $1 \mathrm{~cm}$.

- Allows experimental verification of cooling effects either with circular or slit nozzle and locating the optimum distance between valve and laser beam to maximize sensitivity and selectivity.

- Allows a determination of the effective temperature in the supersonic beam, using either NO or benzene.

- Electrons are removed from the system within the shortest possible time. This reduces possible interaction between electrons and sample and increases the $\mathrm{S} / \mathrm{N}$ ratio.

- Detection of electrons is possible, if desired.

- Reduces interference of the supersonic beam by electrodes. 
- Grid-free electrodes give higher ion transmission.

- Slit formed electrodes in combination with optimized field distribution increase the accessible ionization volume with acceptable mass resolution.

- Incorporates a skimmer in the acceleration electrode to reduce the carrier gas flow into the MS and minimize the interaction time between ions and background gas in the "high pressure" region of the ion source.

- Allows "collinear" arrangement of valve and ion source.

- Minimal exposure of the ions to the surface of the acceleration electrode.

expansion, has been tested previously by others. Although the slit expansion typically cools more slowly than the circular expansion, the fact that the nozzle width is typically narrower than the circular nozzle diameter compensates for this effect. Detailed results from these difficult calculations are not completed.

Based on the promising results of our ion optical simulations for a slit-type geometry, we turned our attention to the details associated with fabricating the various ion optical lens elements required to assemble this device. The primary difficulties in fabricating these lens elements are their small physical dimensions and the very close tolerances that must be maintained to create the required electric field uniformity. We determined that standard mechanical machining approaches could not achieve the required specifications. As an alternative, we explored fabrication methods based on electrodeposition of copper onto a machined form. Following removal of the form by chemical dissolution, the resulting copper lens element would be overcoated first with nickel, then gold.

A vendor was located who could maintain our dimensional specifications, and a number of slit-type electrodes were fabricated using this approach. Sacrificial aluminum forms were machined and plated with copper to the desired thickness. The aluminum forms were then removed by etching in a hot solution of sodium hydroxide. After the copper electrodes are gold coated, they will be assembled into an ion source. This fabrication approach appears to yield copper electrodes of very high quality and dimensional accuracy.

We have begun making modifications to the older TOFMS to accept the slit source. We are currently having the previously fabricated, copper, slit-type ion optics coated with gold. We are also planning to purchase a larger turbomolecular pump of at least $1000 \mathrm{l} / \mathrm{s}$ for the source region, as well as a high vacuum rotatable flange. The former is required to handle the much higher gas load that will be generated using the slit valve, while the latter is necessary to provide exact alignment of the valve with the laser and extraction optics. 
We making modifications to our older TOFMS to accept the slit source, and we have recently purchase a $1300 \mathrm{l} / \mathrm{s}$ turbomolecular pump for the source region, as well as a high vacuum rotatable flange. The former is required to handle the much higher gas load that will be generated using the slit valve, while the latter is necessary to provide exact alignment of the valve with the laser and extraction optics. Both components were acquired using capital investment funds from SRI.

The new ion optics for the slit source have been completed and mounted in the old ion source chamber. The mechanical mount for the slit valve has also been finished, providing both vertical adjustment, as well as rotational alignment. These two degrees of freedom are required to allow us to optimize the signal by adjusting the nozzle-to-laser separation, and the alignment of the jet with respect to the laser to maximize overlap. The final step in preparing the system for testing is mounting of the new $1300 \mathrm{l} / \mathrm{s}$ turbomolecular pump for the source region.

The new ion optics for the slit source had been completed earlier, however, final mounting in the old ion source chamber required several custom fabricated parts. In particular, the low pressure flight tube region must be isolated from the high pressure source region. This isolation is provided by a large collar that surrounds the exit of the ion source with a small ion exit aperture leading to the flight tube. The collar must be electrically insulating and able to withstand elevated temperature, hence it is made from Teflon. A photograph of the new source is included with this report. The final step in preparing the system for testing is the mounting of our new 1300 1/s turbomolecular pump on the source chamber, which also requires some custom fabricated components.

During the course of Phase 2, we determined that General Valve Corporation offers a slittype valve based on the same electromechanical valve driver that we are currently using. Although it is not clear if the new valve design can withstand the elevated temperatures required for our application, it will nonetheless allow us to rapidly assess the impact of the slit design on our sensitivity. We therefore purchased a slit valve and plan to evaluate its performance as soon as the new ion optical system is completed. 


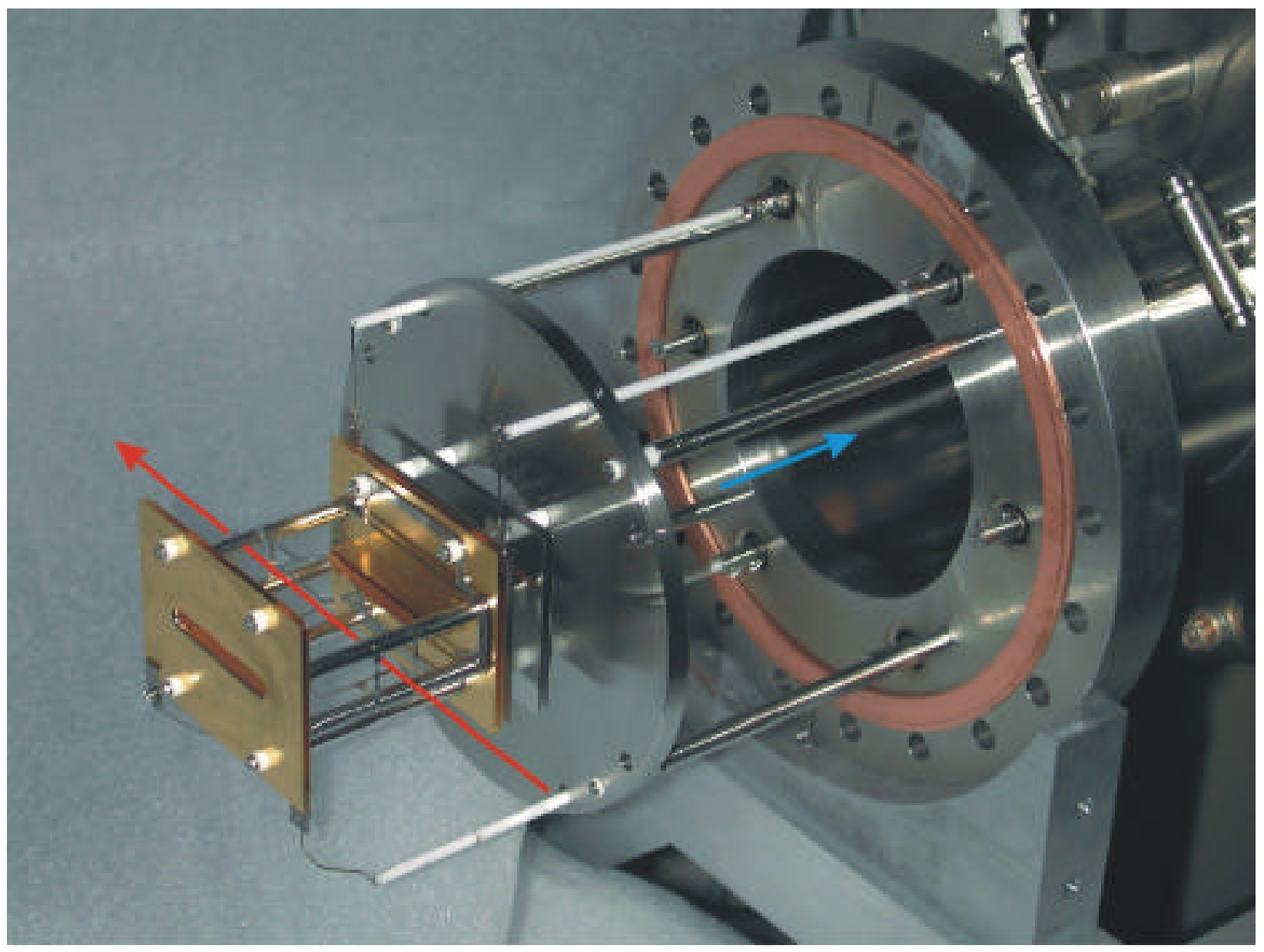

Oblqueview di the SRI stitypeionedraction opdics. The red line shows the approximate laser beam path, while the blue line shows the ion path into the TOF-MS. 


\section{APPENDIX C}

“Development of a jet-REMPI (resonantly enhanced multiphoton ionization) continuous monitor for environmental applications"

Harald Oser, Michael J. Coggiola, Gregory W. Faris, Steve E. Young, Bengt Volquardsen, and David R. Crosley

Applied Optics Vol. 40, No. 6, 859-865

February 2001 


\title{
Development of a jet-REMPI (resonantly enhanced multiphoton ionization) continuous monitor for environmental applications
}

\author{
Haraid Oser, Michael J. Coggiola, Gregory W. Faris, Steve E. Young,
}

Bengt Volquardsen, and David R. Crosiey

\begin{abstract}
The need for a evetimuves monitor foe envionmentally important pollutants at realistie fparta-per trillim parts in $10^{12}$ )] coneontrations moasured in neal time (minutes) is widefy roognizwd. We derel oped an instrument that is based $\mathrm{m}$ enpersmiejos expansion and eocling, followed by resmantly erhaneod multiphotoe imixatice (REMPl) into a nass spoctromoter. This approsech furnishes the dual sobetivity of narrew-hand tuned laser abucorption and mass analysis. Wo initiated a spectroscopic charactorization of the jut's collisioenal enoling behavior to optirniwe the instrument's sensitivity and scbetivity, made mestsaremsats of severnl aromatic cosapounds (inchuding polyehlorinated dinxinsi by use of a cene-color REMPI scheme, and demonstrated a two-oolor exeitation scheme. 5 2001 Optival Socinty of America

OCIS nodes: $910.1120,120.1740$.
\end{abstract}

1. Environmental Needs for a Continuous Emissions Monitor

Eimission-control strategies must ultimately rely on a careful assessment of the link between health effects and ambient, human-exposure levels to hazardous air pollutants (HAP's). Because organic compounds in general and chlorinated aromatics in particular are among the most common toxic HAP's, we are developing a continuous emission monitor (CEM) for these species by using the jet-resonantly enhanced multiphoton ionization (jet--REMPI) technique. Although a wide range of aromatic compounds are toxic polychlorinated dibenzodioxins and polychlorinated dibenzofurans that are emitted from waste incinerators and other combustion sources are of special interest. The capability for real-time detection and identification of these classes of compounds and other toxic $\mathrm{HAP}_{5}$ is an essential component of emissions modeling, dispersion modeling, source apportionment, and, ultimately, of human-exposure modeling.

The authers are with the Molectlar Physics Laboratory, SII Intornational, Monlo Park, California 94025. H. Oser's o-mai addross is harald.cosuretsricom.

Recvived 24 May 2000, revisod manuscript noceived 6 Septombet 2000 .

$000 \mathrm{c}-6935 / 01 / 060859 \mathrm{~N} 07315.00 / 0$

82001 Optical Society of Amorices
Jet-REMPI is the only current instrumental technique that can provide the speed, breadth, and sensitivity that is required to support the development of a comprehensive strategy to monitor and control emissions of HAP's such as dioxins and furans from waste incinerators.

Our technique will provide real-time HAP concentrations (especially for dioxins and furans) rather than the multiple-hour or multiple-day averages required for existing conventional sampling and analysis methods. In the case of dioxin emissions this capability will permit a detailed investigation of the relations among the concentrations of specific isomers and incinerator or furnace operating parameters and waste feed or fuel characteristics. Such a study might reveal that transient pollutant-emission events account for a majority of the dioxin emissions; hence only short-term control methods would be re. quired to meet time-averaged regulatory emission limits. Such control strategies may be much more cost effective than full-time emission-control approaches. Tailoring the emission-control strategy in response to minute-by-minute changes in the emission of hazardous pollutants can be accomplished only if a suitable CEM is available.

For urban air-pollution studies the speed and the versatility of jet--REMPI will allow both the spatial and the temporal distribution of a majority of the most toxic organic HAP's to be measured concur- 
rently at levels of toxicological interest. Because our CEM is capable of directly measuring, in real time, the concentrations of specific HAP's and urban air pollutants at levels far below many present real-time analytical instruments, we will have the capability to identify and characterize critical emission sources over a wide ceographical area under a variety of ambient conditions. Spatial and temporal mapping of HAP levels as functions of meteorolorical, seasonal, and atmospheric conditions will establish a means of identífying emissions trends, determining humanexposure levels, and assessing the increased risks associated with specific geographical areas or subpopulations. Also, it will permit experiments to determine rapidly the cause-and-effect relations between HAP emissions and the operational conditions of their sources.

2. Jet-Resonantly Enhanced Multiphoton Ionization as an Anatytical Tool

Our approach to acquiring the data on HAP levels is the direct measurement of specific compounds by use of the jet-REMPI method. Jet-REMPI is an ultrasensitive analytical technique that can selectively identify and quantify vapor-phase constituents present at part-per-trillion (ppt, or parts in $10^{12}$ ) levels directly in air samples. For this reason, we do not use sample-preconcentration techniques.

In recent years many significant improwements have been made in the detection of hazardous, vaporphase, oryanic compounds of environmental importance. Several research groups, including ours, ${ }^{1}$ the Deutches Zentrum fuer Laft- und Raumfahrt, ${ }^{2}$ and especially The Technical University of Munich, ${ }^{3} \mathrm{Ger}-$ many, have demonstrated analytical instruments based on REMPI and mass spectrometry.

Ions produced by REMPI are detected by use of a time-of-flight (TOF) mass spectrometer (TOF-MS) that takes advantage of the pulsed nature and the well-defined temporal character of laser ionization. Simultaneous detection by mass and wavelength yields the extremely high chemical selectivity that is crucial to identifying one trace compound in the midst of many other similar ones. One photon resonantly excites the molecule in the lowest singlet to the singlet transition $S_{1} \leqslant S_{0}$, yielding selective fingerprint spectra that involve individual quantum levels in each state. The second step is photononization from $S_{1}$. This step produces little or no photofragmentation, so usually only the parent molecular ion is formed, allowing easy identification through TOFMS. The resonant step is usually saturated, and more than $10 \%$ of the sample is converted to ions in each pulse.

We wish to use jet-REMPI to distinguish a trace species in the midst of many similar compounds. This use of jet-REMPI as an analytical tool contrasts sharply with the vast majority of previous experiments with the technique in which the spectra of individual compounds were stadied one at a time and at moch higher concentrations. This analytical approach requires careful attention to the resolution

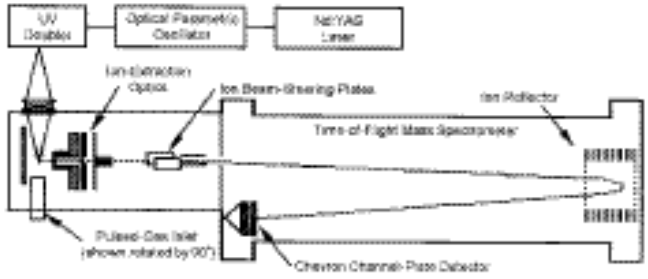

Fig. 1. Schomatic of the jot-REMPI apparatus devoloped at SRI.

characteristics of both the laser-excitation step and the mass measurement.

Figure 1 shows a diagram of the laboratory jetREMPI TOF-MS instrument that we assembled at SRI for measuring HAP's. Instrumental details are given below in Subsection 2.D.

A major improvement in sensitivity without a loss in selectivity can be achieved by nse of a pulsed gas valve that produces a supersonic expansion. Pulsed gas valves provide a number of advantages over continuous gas inlets, including a reduced gas flow (hence smaller vacuum pumps), higher local gas densities, a well-defined spatial distribution, a significantly reduoed translational energy distribution that is orthogonal to the propagation direction, and reduced internal (vibrational and rotational) temperatures, leading to a greatly enhanced spectrosoopic resolution.

REMPI is a highly sensitive, highly speciesselective, gas-phase analysis technique that has been applied to numerous problems in molecular spectroseopy and combustion research. For the REMPI of complex molecules, such as dioxins and other polychlorinated aromatics, the spectra can be simplified greatly by expansion through a nozzle. Adiabatic expansion results in low sample temperatures, which increases the rotational and the vibrational groundstate populations of the electronic ground state. It also narrows the resonance linewidths through a reduction in molecular velocities (reduced Doppler or temperature broadening) and through a reduction in transition-perturting collisions (reduced pressure broadeningy. These reduced linewidths lessen the excitation of other molecular species in the same wavelength region (interferences)-leading to improved selectivity-and make the peak absorption larger-leading to improved sensitivity. The same or a separate wavelength is used to ionize the cooled sas molecules by the absorption of two or more photons, one of which represents the resonant-excitation step.

\section{A. Laser-Excitation Considerations}

Because different isomers of a given chemical composition can have very different toxicities, it is essential that a measurement instrument be capable of distinguishing among isomers. This requirement is particularly true of dioxins and furans for which very closely related isomers exhibit vastly differing toxic- 
ities. For example, the 2,3,7,8-tetrachlorinated dibenzodioxin (TCDD) is one of the most toxic compounds known, whereas the $1,3,7,8$-TCDD is relatively benign. Our instrument is able to identify closely related species without any sample preseparation (as in gas chromatography, for example) because of the inherent capability of optical spectroscopy (REMPI in this case) to distinguish readily among isomers. Supersonic cooling is critical to obtain this selectivity from optical spectroscopy.

One or two wayelengths can be used for ionization with REMPI. One-color REMPI is simpler to implement. However, in certain cases using a second color is preferable or essential. For example, if the first excited state lies lower than half of the ionization energy the excitation photon will not have enough energy to ionize the molecule. For TCDD the energy of the first excited level is approximately half of the ionization potential." Thus single-color REMPI may or may not be effective for TCDD. Two-color REMPI that uses a second higher-energy laser beam may be a more efficient technique for TCDD detection. The second beam need not require an additional laser. For example, when using a tunable Nd:YAG pumped laser the second color can be the fourth or the fifth harmonic of the Nd:YAG fundamental produced by use of nonlinear frequency conversion.

Resching the first excited state of most chlorinated aromatics requires a laser wavelength in the range of 250 to $350 \mathrm{~nm}$. We produce this wavelength with a frequency-doubled optical parametric ascillator $(O P O)$ pumped by the third harmonic of a Nd:YAG laser.

\section{B. Mass-Spectrometer Characteristics}

The mass spectrometric requirements of a CEM instrument are modest: an upper-mass range of 400 with unit mass resolution is sufficient for HAP monitoring. Typical TOF mass analyzers can meet these requirements by use of a simple linear flight path when combined with a short-duration, pulsed-laser, ionization source. The low signal levels that can result from the use of a narrow ion-formation pulse can be compensated for by use of signal averaging with multiple laser pulses. Achieving high mass resolution with a TOF instrument is straightforward when using pulsed-laser ionization because of the narrow spatial, temporal, and energy spreads of the nascent ions.

An increasingly common TOF approach is the use of an ion mirror, or reflectron-type, TOF. The ion beam is reflected by a potential gradient (an electrostatic mirror) that compensates for small differences in the ion velocities and thus better resolves the mass spectra. The properties of this device are well known, and a number of computer codes have been developed to assist with their design. Resolving powers $(m / \Delta m$, where $m$ is the mass of the detected ions) of more than 1000 can be obtained readily by use of a reflectron with a pulsed-laser ionization source. Although mass-resolving powers in excess of 1000 are not necessary to separate adjacent mass peaks even at the highest mass-to-charge ratios expected in an environmental CEM, high resolution gives rise to signals with much narrower-hence much higher-peaks. The increased peak amplitude leads directly to an increased signal-to-noise ratio.

A significant improvement in the resolution and the signal levels can also be obtained through the careful design of the ion-extraction optics, As a result of the pulsed-laser ionization scheme, ion formation is eonstrained to occur over a very limited spatial region. Normally, a well-constrained ion-formation region can readily be coupled with high efficiency to a TOF mass analyzer by use of simple ion-extraction optics. In this instrument, however, an additional spatial constraint is imposed by the use of a pulsed gas inlet. An optimum location exists downstream from the orifice where the internal molecular temperature will have reached a minimum, while the local gas density remains relatively high. Ionization at this location will yield the optimum optical spectroscopic selectivity with the maximum ion signal level. Because this optimum ionization region will typically be only a few centimeters from the exit orifice, the ion optics must be designed carefully to include the influence of the pulsed valve on the extraction fields.

\section{Two-Dimensional Detection Scheme}

For jet-REMPI to serve as an analytical tool, we must have available a library of the resonant excitation spectra for both the target compounds and the potential interferents. For example, one may wish to detect five of the most toxic dioxins within a waste incinerator's flue gas, which contains many similar molecules, especially different isomers of the multichlorinated compounds. A cement kiln, which also is a dioxin source, may have a very different interference condition. Building this library up is a major part of the development of jet-REMPI for environmental applications. We currently are developing software to automate and speed the process so that the instrument-control computer can select the turing range and the mass for a given compound and identify potential interferents. This software will take full advantage of the two-dimensional selectivity, wavelength, and mass afforded by jet-REMPI. Because the full mass spectrum can be captured in each laser pulse, several compounds can be determined at the same time if their absorption spectra overlap in the same region.

The two-dimensional nature of jet-REMPI is illustrated in Fig. 2. The ordinate of Fig. 2 corresponds to the parent-ion mass of a number of common HAP's, whereas the abscissa corresponds to the resonant $S_{1}$ $-S_{0}$ transition wavelength. It is apparent that most HAP's are well separated in this twodimensional space, thus allowing unambignous detection and identification by jet-REMPI. There are, however, several instances of two or more HAP's appearing nearly coincidently in Fig. 2. For example, in the upper right-hand corner of Fig. 2 the 2,3- 


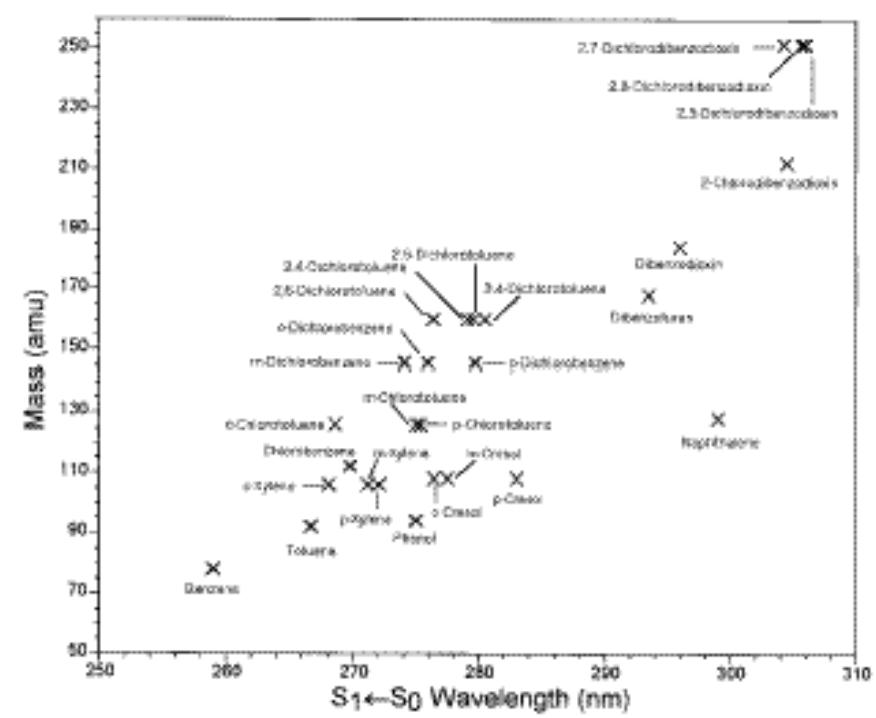

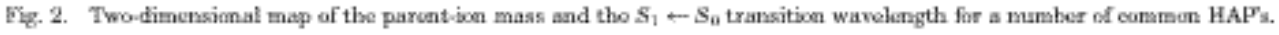

dicholorodibenzodioxin (DCDD) and the 2,8-DCDD both appear to have a nominal parent-ion mass of 252 amu and an $S_{1} \leftarrow S_{0}$ transition at $306 \mathrm{~nm}$. An expanded view of this region is shown in Fig. 3.

The higher-resolution plot in Fig. 3 shows that there are four dioxins with nearly the same parention mass and transition wavelength. Both the $2,3-$ DCDD and the 2,8-DCDD have multiple isomers arising from the two chlorine isomers ${ }^{35} \mathrm{Cl}$ and ${ }^{37} \mathrm{Cl}$ that are present in a ratio of approximately 3:1. Although each oompound has three chlorine isomeric variations, only the two most abundant are shown in Fig. 3. For simplicity, we ignored all other isomeric species, including those resulting from ${ }^{13} \mathrm{C},{ }^{2} \mathrm{H}$, and the isotopes of oxygen. In the expanded mass-wavelength region of Fig. 3 the four chlorine isomers are readily separable in both dimensions. This separability is illustrated also by the TOF-MS resolution

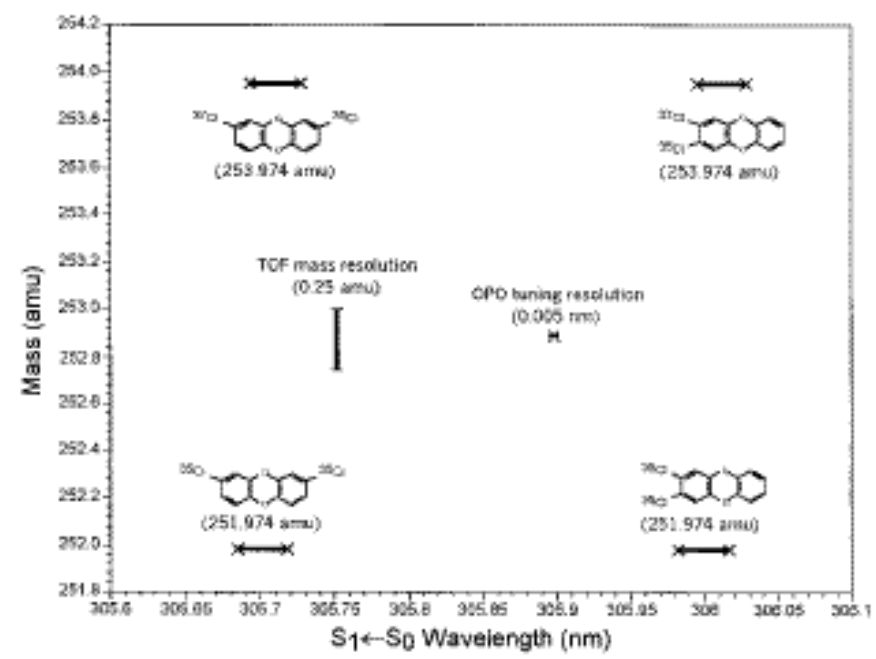

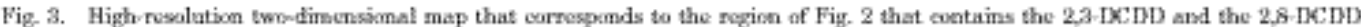
isomors. The instrumenisl mass and the wavelength resalutions aro also shown. 
(approximately $0.25 \mathrm{amu}$ ) and the wavelength resolution provided by the narrow-band OPO (approximately $0.005 \mathrm{~nm}$ ). The latter resolution is sufficient for the observation of the slight wavelength shift in each isomer that is due to the heavier chlorine isotopes. The observed 0.03-nm width of the $S_{1} \leftarrow S_{0}$ transition for each isomer is indicated by the horizontal bar.

\section{Specific Detalls}

The laser system used in these experiments consists of a Continunm Model Powerlite Precision 9010 Nd: YAG pump laser and a Model Sunlite EX OPO with a nominal tuning range in the visible between 445 and $710 \mathrm{~nm}$, a 5 -ns pulse width, and a repetition rate of $10 \mathrm{~Hz}$. Frequency doubling is achieved by use of a Continuum Model FX-1 option. The optical linewidth of the system is approximately $0.2 \mathrm{~cm}^{-1}$ in the UV.

The pulsed valve is an unmodified General Valve series 9 unit with an orifice diameter of $0.5 \mathrm{~mm}$ and a $2.5-\mathrm{cm}$ separation between the exit of the valve and the ionization region. The nominal opening time is $150 \mu \mathrm{s}$, which admits approximately $2 \mu \mathrm{l}$ of gas per pulse, the vast majority of which is carrier gas. With the sample reservoir at atmospheric pressure two 250-1/s turbomolecular pumps (Varian, Model Turbo $\mathrm{V}-250$ ) maintain pressures in the ionization chamber and the mass-spectrometer regions of $10^{-1}$ Torr and $5 \times 10^{-7}$ Torr, respectively.

The ionization conditions used to acquire the data included a pulse energy of approximately $1 \mathrm{~mJ}$ with a $2 \mathrm{~mm} \times 2 \mathrm{~mm}$ active beam area. The wavelengthdependent spectra were measured with an optical resolution of $0.002 \mathrm{~nm}$, and 30 laser shots were averaged per wavelength step. A wavelengthdependent spectrum such as that shown in Fig. 5 below thus required $100 \mathrm{~min}$ to acquire. The lasercreated ions were mass analyzed by an R. M. Jordan reflectron TOF-MS with a mass resolution of approximately 500 . The ion signals were amplified by an Ortec Model 9306 preamplifier with a gain of 85 and a $1-\mathrm{GHz}$ bandwidth and recorded by a $500-\mathrm{MHz}$ Signatec Model DA500A digitizer.

\section{Discussion of the Experiment}

\section{A. Rotatiansl Temperature}

The effective temperature of samples in the supersonic jet is important for both sensitivity and selectivity with jet-REMPI. To learn the operating conditions for obtaining the lowest temperatures from the pulsed valve, we used benzene as a thermometric species. The spectroscopy of benzene is quite well known, which allows the determination of the effective rotational temperature from measured spectra.

Measurements of a single-band contour for benzene are shown in Fig. A/a). This spectrum was taken at a nozzle-to-mass spectrometer center-line separation of $2.75 \pm 0.05 \mathrm{~cm}$. The band shown is part of the $S_{1} \leftarrow S_{0}$ transition of benzene near $259 \mathrm{~nm}$

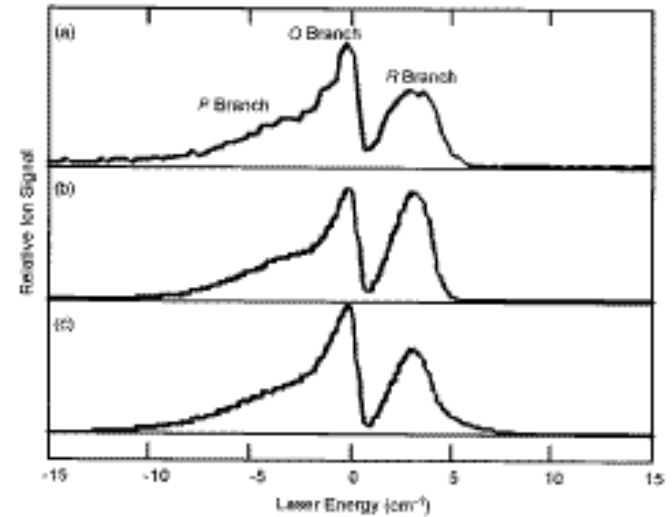

Fig- 4. Rolative ion signal and spectrosoopic fittings for the ' $B_{2}$ $-{ }^{-} A_{1}, B_{0}^{2}$ transition in benzone. Shown are $(a)$ the moasured REMPI data and calculatod fittings to tho dala (b) with the as. sumption of ne saturation and fei with the assunaption of eomplote saturation of the individual rotatiosal lines. The center of the transition band that was investigatod was 38 fo6 $\mathrm{cm}^{-1}$ and was cuntured at wro on the laser.onergy coordinate.

with a spectroscopic identification of ${ }^{1} B_{2}-{ }^{1} A_{1}, 66_{0}^{1}$. The band shown ouresponds to many overlapping rotational lines. From the overall contour of these overlapping lines, we can determine an effective rotational temperature of the benzene molecules.

We present preliminary results from a fitting prosram that was written to simulate the form of the band contour as a function of temperature. The rotational-band calculations follow the method given by Callomon et al, ". We use literature values for the spectroscopic constants, ${ }^{6}$ appropriate statistical weights for the ground-state populations, ${ }^{7}$ and the appropriate Honl-London factors, ${ }^{3}$ which describe the rotational dependence of the transition probabilities. The results from this fitting program are shown in Fig. A(b). The general shapes of the $Q$ and the $P$ branches agree well with the experiment, but in comparison with the data the $R$ branch is too large relative to the other two branches. Becanse the first. step of the $1+1$ REMPI prooess, i.e., a two-photon excitation-ionization process with photons of the same wavelength, is likely to be saturated, we performed a second fitting by assuming that all the transitions were fully saturated (i.e, the Honl-London factors were all set equal to 1). The result of this fitting is shown in Fig. 4(c). This fitting yields a much better agreement with the data than does the fitting shown in Fig. 4(b). The resultant temperatures are $20 \pm 1 \mathrm{~K}$ and $19 \pm 1 \mathrm{~K}$ for the fittings shown in Figs. 4(b) and 4(c), respectively.

\section{B. Selective Determination of Isomers}

We demonstrated both isomer selectivity and instrumental sensitivity by using a sample that consisted of a mixture of equal amounts of 2,7-DCDD and 2,8- 


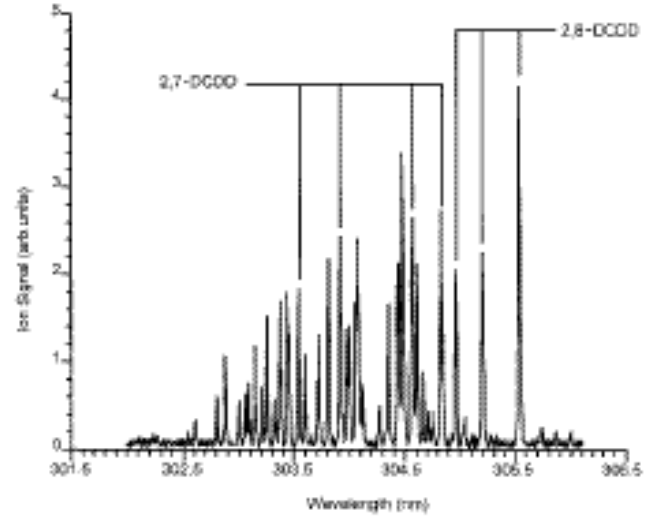

Fig. 5. Jet-REMPI wavelongth dopundenoo for an equimolat mixture of 2,7-DCDD and 2,5-DCDD measured at $m / z=252$.

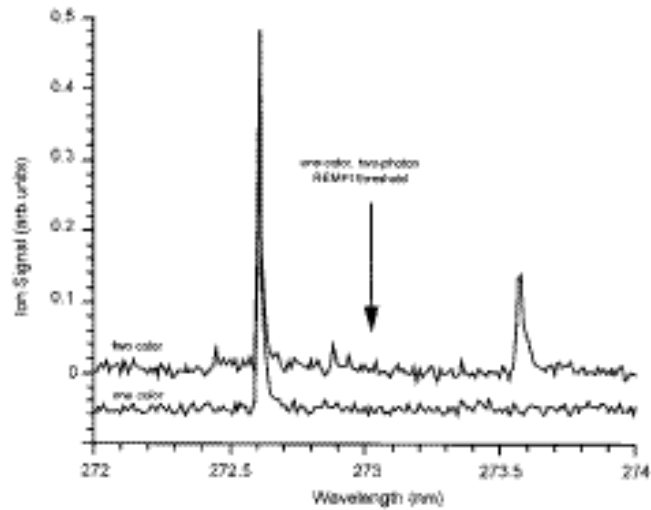

Fig 6. Wavolongth depondence of the $m / z=146$ ion signal fine 1,2-dichlorvbonzene revorded by use of both a coe-color, twophoton oxcitation-innization schems and a two-ooles, two-pboton REMPI schome.

DCDD. To simplify these tests, we injected a small quantity of the liquid mixture into a stainless-steel sample flask on the upstream side of the pulsed valve and used room air as the carrier gas. We estimate that the concentration of each isomer in the sampled gas stream was approximately 1 ppb ( 1 part in $10^{5}$ ). All components in the sample stream, including the nozzle, were heated to $150^{\circ} \mathrm{C}$ to prevent condensation.

Figure 5 shows the ion signal recorded at $252 \mathrm{amu}$ that corres ponds to the parent ions of both isomers as a function of wavelength. Several individual absorption features are labeled to show which isomer gave rise to the ion signal. Assignment of these absorption features was made by the messurement of the jet-REMPI spectra for each isomer separately ${ }^{2}$ and by comparison with the results of Weickardit et $a l$ " From an estimated signal-to-noise ratio of 20, we derived a current detection limit of 50 ppt for both investigated DCDD isomers.

\section{Two-Wavelength Resonantly Enhenced Multiphoton} ionization

For many molecules of interest, particulariy tetrachlorinated and higher dioxins and furans, the onecolor, two-photon REMPI excitation and ionization scheme is difficult or impossible to use because the first excited state lies less than half the distance to the ionization limit. For those molecules a two-color REMPI scheme must be ased in which a tunable wavelength is used to excite the first excited state resonantly and a second fixed wawelength is used to achieve ionization. To demonstrate the two-color approach, we examined the jet-REMPI wavelength dependence for 1,2-dichlorobenzene,

Figure 6 shows the $m / 2=146$ ion signal measured by use of a combination of tunable UV from the OPO and $266 \mathrm{~nm}$ produced by the quadrupling of the fundamental wavelength of the Nd:YAG pump laser. Because 1,2-dichlorobenzene can be ionized from higher rovibronic levels of $S$, by the one-color, twophoton scheme, we show that ion signal in Fig. 6 as well. Note that the large absorption peak at 272.61 $\mathrm{nm}$ appears when both ionization schemes are used. However, the absorption peak at $273.58 \mathrm{~nm}$ appears in only the two-color signal. This feature arises from the absorption of one photon that is resonant with vibrationally excited molecules in the $S_{i}$ state and followed by the absorption of a 266-nm photon to produce ionization. Because the absorption of a second photon at $273.58 \mathrm{~nm}$ cannot ionize 1,2dichlorobenzene, this feature does not appear in the one-color REMPI spectrum. As shown in Fig. 6, the threshold for the one-color, two-photon process is approximately $273 \mathrm{~nm}$ between these two features.

\section{Conclusions}

We have demonstrated a prototype HAP monitor that is based on the jet-REMPI technique. A preliminary spectroscopic investigation of the molecular cooling produced in the supersonic gas expansion indicates a high degree of rotational relaxation. The detection and the identification of two closely related isomers of dichlorinated dioxins in a mixture at the 50-ppt level has been achieved by use of this instrument. Furthermore, we have demonstrated the application of two-color REMPI for the detection of chlorinated organic molecules.

This study was supported by the Federal Energy Technology Center, Department of Enerny, under contract DE-AC26-98FT-40370 and the U.S. Environmental Protection Amency under srant R82792701. The anthors would like to acknowledge the assistance of Katherine Copic in developing the data-acyuisition and the ontrol sottware. 


\section{References}

1. H. Oser, K. Copic, M. S. Coggiola, G. W. Faris, and D. R. Crosley, "Congenor spotific detoetion of dioxins using jet-REMPI," Che mosphere ito bo pablishod $)$.

2. H. Oser, R. Thanner, and H. H. Grothoer, Het.REMPI Sor the doloetice of trace gas oompounds in exmplex gas mixturos, a tool for kinotic research and inciberatar process eventrol," Combast Sci. TothnoL 116, 587-572 (1996)

A. C. Woickhardt, U. Boosl, and E. W. Schlag, "Laser mass speoe trometry for time rossalved mulitioomponent analysis of oxhaus gas, 'Anal Chom. 66, 1062-1069 (1994).

4. F. Zimmermann, D. Lonoir, A. Kettrup, H. Nagul, and U. Boesh. On line umissim control of eambeastion procosses by lasor indused resosaneo-cahanood multiphoten imization-mass spectrumotry," in Proesedings of the Tiventy-Sixth International Symposium on Combustion fThe Combustim lnstitute, Pitts burgh, Pa., 1996\%, p. 2869.
5. J. H. Callornce, T. M. Dumn, and 1. M. Mille, "Futational analysis of the 2800 . $\hat{A}$ ahsorption system of honaene," Thilos. Trank. R. Soc. Lundin Ser. A 259 , 499 552 (1988).

6. ML Oaruss, E. Malber, and A. Hese, "High resolution UV laser

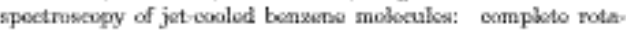
tional analysis of the $S_{1}-S_{0} G_{0}^{2}(1= \pm 1$ h hand,", . Mol. Spoctrose. 198, 29:3 za5 (1999).

7. E. B. Wilsun, Jr., "Statistieal weights of the rotational kwols of polyatomie moleculos, including methane, a mamania, benzene, usckgroguans, and sthylone" 3. Chem. Phys. 3, 276-285 (1935).

8. G. Herzberg, Moleculas Spertrn and Molecular Structure (Van Nostrand, Now Yark, 1966), Vol. III, p. 292.

9. C. Woickhardt, R. Zimmormann, K. W. Schrumm, D. Lonvir, U. Boosl, and E. W. Schlag, "Laseor mass epoctromotry of dibcrono" dioxin, dibonzofiuran, and two isomors of dichlorodibenzodiax. ins: solutive ionization," Rapid Conmmun. Mass Spectrosn. 7. [8] $187(1998)$. 


\section{APPENDIX D:}

"Congener-specific detection of dioxins using jet-REMPI"

Harald Oser, Kathy Copic, Michael J. Coggiola, Gregory W. Faris, and David R. Crosley

Chemosphere Vol. 43, 469-477, 2001 


\title{
Congener-specific detection of dioxins using jet-REMPI
}

\author{
Harald Oser ${ }^{\text {a, }}$, Kathy Copic ${ }^{\text {", }}$, Michacl J. Coggiola ${ }^{\text {, }}$, Gregory W. Faris a", \\ Dayid R. Crosley ${ }^{\text {a }}$

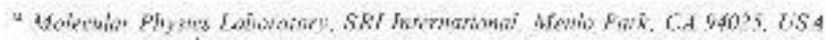

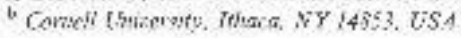

\begin{abstract}
Although 210 chemically different polychlorinatod dibenzo-p-dioxin and dibsnzofuran congeners can be produced during conbustion, it is currently believed that fewer then 20 ale toxic cnough to warrant monitoring. SRI is developirg a continuous cmissions monitns to study the emission levels of chese most toxis dioxins, leading eventually to an improved understanding of the formation of thess molecules and to improved means nf monitoring and control, We report here the first results of performing engener-specific detection for two dichloro ditengo p-dioxins present in low ppeconecntrations in a mixture using the supersonic jetresenantly enhanced multiphoton ionization time of flight mass

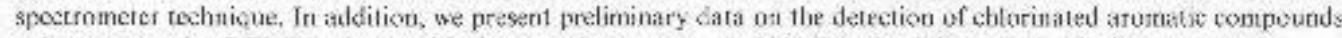
using a rwo-color RFMT whente with the same irstrument. 92001 Elsevies Sciente L ki All righls reserved.
\end{abstract}

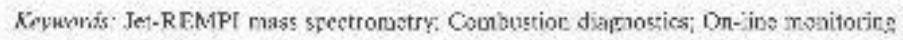

\section{Introdintion}

The need for in continuous emussons monitor (CEM) for the deternination of polyctlonnated dibenco-p-dt. oxins (PCDDs) and diberzofurans (PCDIis) al realistx cuncentrations (parts per trilion or lower) in real tume (minutes) is widely recogmzos. Such an zostrument would enable rupid ans reul-time experinentation on incinerators, so at to test and develop control strategies. It would also be extremely usetul in laboratory kinetics expenments seeking to understand formation. interconrersion. and destruction processes for dicxius and theis precursors. lijualty, it could be used eventually tor continuous conpliance monitoring, or rapid sad lowcost facilities cert:tication. The key issues for 2 dioxin CLM are: overall sensitivity. selectivity among the many congeners tound in real applications. and the ability to

"Carrespanting iuthar. Fax: $+1-650-859-6195$.

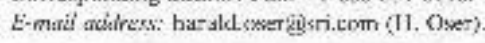

detect and distingush these compounds in the presence of a very womplex anckgrounci.

Our approach for the real-time djoxin CLM cotnbires a pulseat gas jer wath resonantly enbanced multiphoton ionization (RLMPI) and tome-of-flight mass spectrometry (TOLMS). This article describes our initjal demonstration of a laboratory instrument that can provide contimuous monitoring at the enission levels of PCDDil's generated by inciserators. Onos developed, this inst:ument will be usot to systeruaticaly study the enission levels of the key dioxins and furuds that contribute to the toxic equivalency (TEQ). This infor. nation, combinod with mecianistic modeling studies being undertaken elseckihere (Guilet, 1997). will keid tn the design specizications for a real-time, auloumonos dioxin CEM that can $x$ used for system control, and eventually compliance monitoring, at wuste inemerators.

A reasonable tirst use of a dioxin CEM is as a icsearch tool in laboratorics studying dioxin formation and oontrol (Oser et al., 1996a). As such, the itstrumeut must make rapid, socurate measurementis of 
dioxins but al cuncentrattions much higher than necded for a curapliance CEM. A laborator? instrument will gevaty accelerale our understanding of thusin formation and the deusibilty of mplestentug prevettion and ontrol tochaiques. Researchers tave u fimiled under standing of how combustion procerses alleet dioxim formation. Due to the lack of real-fone monters, current approaches reguirs relating t:me-ialegrated sampling data with dynamk formutwon metbarusros and onmlsustor conditions after un enalysis dug periox that is often many wecks. A real-time CEMt will provide immediate feedhack on hon variations in conshustion opecisting parameters afect dioxin formation andior destutetina. thus allowing more accurate co:relations and far more comprehensise data analysic. $A$ real-tine dioxia moaitor knuld also be valuable as nees waste trealnseal procesw:s alc evaluated and readiox for pernillingipublic ateseptanos.

Current techeliyurs lu masitor emissions of $\mathrm{PCOD}_{3}$ and PCDDis use sampling limes in exeess of hours. durisg whath the antalyues are collecled on adsorbiag materials followed by sampie exiraiction and preparation tor subsequent gas chirumalographyimase spectrometry (GCJMS) analysis. These are custly and time sonsuming methods whose crawbicks Jead to compliance measurements made only infrevuent; (perhaps onse or twice per year). The consejuences atre regul i wry strategies that rely on indirect process monituring sather than dirost mozitoting and dioxir preverntion strategies, often Jeading to inettisieut air pottuition con. trol systems.

As ou: understanding of PCDD and PCDI formation improves, it would be valuable to buld a dutabase using emissions from actual waste treatmen! processes and correlate operating conditions with PCDD and PCDF formation. Such a database could be used to devise operating strategics to prevent tormation of PCDD and PCDF. This database could ulso be used in altempts to identify surrogates or indicutors that an be munitorel more easily and cheaply than the PCDD and PCDF thernselves, leading to less expensive, more widely implemented complianos and control stratepies,

\section{Sunsitivity requirematnts}

Toxw equivalence factors (IEt) are defined as a se: of wesghting factors expressios foxicity of eath enanpound relative to the loxicily of $2,3,2,8$ telrachlorodibenzodioxin (ICDD) TLIs vary fron 1.0 to 0.001 for 17 different polychlorinated compounds. Fach of these 17 waneners cunlains chlorine atoms ia the $2,3,7$, and 8 posjtsons, 16 having additjonial chlorine substitutions at one or more olber positions. The solat loxic equivalenes (TEQ) is calculated by multiplynge the cunceatiation of cach PCDD and PCDI by its curresponding TLE factor. Several wejehting swhemes sre ivalable. NAIO has proposed a scieme assignire tactors to the 17 taosl woxic polychlorinated dioxins and furans, and ussigning no factor 25 the remaining $1 \% 3$ zengeners. Estimates of performance requirements for a dioxin CEM for regulatnry compliance can be made kased on the existing knowiedge of TEF and TEQ. Considering only she 17 compends in the total TEQ. the sensitivity required for is diexin C.M would noed $w$ be a factor of nerbaps ten ess ibata the emissien limit for cach of the 17 congeners ij 5 , sivide the TEQ by $12,10 \%$. Basod on the reontly promulgated maximuru achievable control wholhology (MAC:I) rules, the mosinum TFQ is set at $0.2 \mathrm{ng} \mathrm{gm}^{3}$. Hence, the rejuired minimu derection timit is covikaJent to the ernissun limil divided by 17n, $\mathrm{mi}$

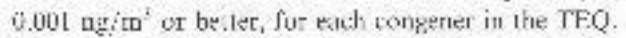

In the bes case, if you ceuld argue thal ibe proba bility of formution of eatch wergenter was abual equal, then the minmun detection limets could be hirber fur compounds with a lower TLQ fuctor. In cher wends. given a minimun detection limil of $0001 \mathrm{ng}^{\prime} \mathrm{m}^{3}$ fur 2.3,7.8 TCDD. the other I6 PCDDiI congeatrs having TF.Fs berwoen 0.5 ard 0.001 would huve correspordag minimum dercetion limits of $0.002-1.0 \mathrm{ng} / \mathrm{m}^{\prime}$.

As shown in the cstimetes atwove. the detection liruts mist be very law to dircetly measure all 17 congeners an th. TFQ. A proposed alocrnative is to monitor prevussters or alurogates. identiiied usirg a true PCDDiI CLM in reveatel laborawries. This real-time dutu woth be invaluable to develop mechanistic understandings, Ir:adiag, in surrogates of preceusors that can $b$ medsured al much thigher conceatrations. For exemple, it has beer segresteal (Gullert, 1997) that the study of lightly thlorinaled (muno-, di-, ur tri-) dioxins may be acceptabie. A so, these wongeters are casicr to measure than the more bighly chlorinated dioxins. The molocules are very similar to those in the TFQ and may xell kead to correlations describing the furmation of TFQ oongeners.

Laboritory experiments for sladying dioxin formation and wotrol usually operite at wat dioxin onncontrations around $100 \mathrm{l}-1000 \mathrm{~g} / \mathrm{m}^{3}$. Degending on how: many of the 210 tolal congeners ; are present, detection limits around $5 \mathrm{ngmi}^{3}$ for eich compener of interest would be more than adejuate. The first step in developing a dioxan CLM suitatble for compliance moniroring is. therefore, to develop u Jaboratory instrument capahle of speciating dioxin congeners, ankior precursors, of strrogates, and meusuring ther wountratiurs in iealtime xith a sensitivity in the low ng/ $\mathrm{nt}^{3}$ range. By conbinire this information with sophisticated combuston models, it will te porssble to determine the spccific target chenusenls that nusi be measured by is dioxin CLM, and the levels to which they must be measumod. Only after the intoroation is is widiatble tan a dioxin CEM suitable tor complanove use be develonest. 


\section{Insirumerutation}

In reoent ycars, many sjenificant improvements have been made in the detection of dinxins resultiag from harious combustion processes. Several isseacl zteups, incleding SRI (Oser el at., 1\%g9), Deutsele Forstury sunstalt fur Luft und Ruunduhet (DI.RI in Srullzirl Germany iOser et all. 1\%\%6, 1997, The Texunical University of Muriwh, Germeny (Weickhitrsht el al 1993: Zimmemarus el al.. 1995), and Sirrulit Natjoral Lahoratories (Roblting. 1988) in I.ivermore, f.A. bave demnnstrated stack menitoring instrumets or components of instruments hased on RFMPI. lo date, horever, bute of these rescarch progratns has acbueved the sensitivily reyajed ter either comp iance montoring or s rescarch CF $\mathrm{M}$ to study formation and conleol of dioxias.

Or partucular interest and promise for the real-time chanaterizution of dinxins is the combination of a pulsed gals jet witk. RF.Mाग and turue-of-tight mass spectrometry (TOFM.S). Iising itis upproach, the group at DLR Stuttgart has measured non-, mono-, di-, tir-; and tetru-chlotinatod diexirs at the LPA National Risd Magagement Laboratniy (NRML) tacility during wois from July to Oernher i 996 ; Bergan French et ai., 1997), The measured minimum detectability for dicllorirntel dioxins was approximately $20 \mathrm{ng}_{\mathrm{z}} \mathrm{m}^{3}$. For the trithlorodibenzodinxin and letructlorosibenzodioxia, the detection limits were not measurod. but they hase boe

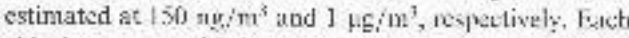
chlorine atom atcied to the molocule secrns to riske detoction hander assugr the current, one-wolkn, lwev-photon, inaization method. For this teason, we are deve:oping fso-culur, (wo-photon, ionization methods that will impove the detectability of the mure figthy chlorinated tlioxims.

RFMII will aoed significant entharlecnerts to reach. the suh-ny: $m^{3}$ detection limits required for a compliuno or systern control CEM. As a reseirch dioxin CEM, huwever, the edrsem REMPP-1OI MS scheme may need to be improved by only one or two orders of magnitude.

SRI las heen using rutuphoton-mass apeetronetne tohrigues to study spertustopy and traos-lesel detestion for more than it deade. We originally appled theso methods to the deletion of uromatic and chlorinated organic com-xurics \& Rossi and Eckstrom, 1985; Resst and Heln, 1987). lior RLMPI of complex molexules. such as diusins, tbe syertza ean ine simplified greatly by expandion ib-uath a nozzlc. Adiahar expinsion result: in low sumple tengeratures, a few degres: $\mathrm{K}$, which iacreases the elextronic ground stitte population and nanows the resonane line widthe. The enbanos popLlation of tbe ground state gives an increilse in sensitivity, while the narrower line willth give rise to very shan RLMPI transition. One or wo lusers are used to innize the coolod gas molecules by atborption of two or uore pho:ons, one of which is nesonant with an clectrunic transition in the targel molecule.

lons produced lo REMPI are often detected using a TOI-MS that takes acrantiuge of the pulsed nature and xell-definod cemporal thancter of laser ionizatina. Many REMPI experineats pertormed at SRI and ylsewhere have acetessfillty used the TOF-MS amproich The simultaneous detection by mass and waveleryth yields higl olernical selectivity. Typical sensitivities of conventional RFMPI TOL -MS syatems that do nut use sample molecule couting are in the mg/ $\mathrm{m}^{3}$ range and higher, which is greatly josulficient sither for a lahuratory system or for regulatory menitoring of elloriraled aromatics in as welste treutment facility such as ist in ciacrator:

Fig. 1 is a diayum of the laboratory protolype RlMPI TOF-MS isstrument (Crosley et al., 1993) that we have asvembled at SR1 for measuring PCDDiF:

Puised kabe-trpe gas iniets have beea useal in a wide sariety of mass speetrometric and laser spec:lrowop:

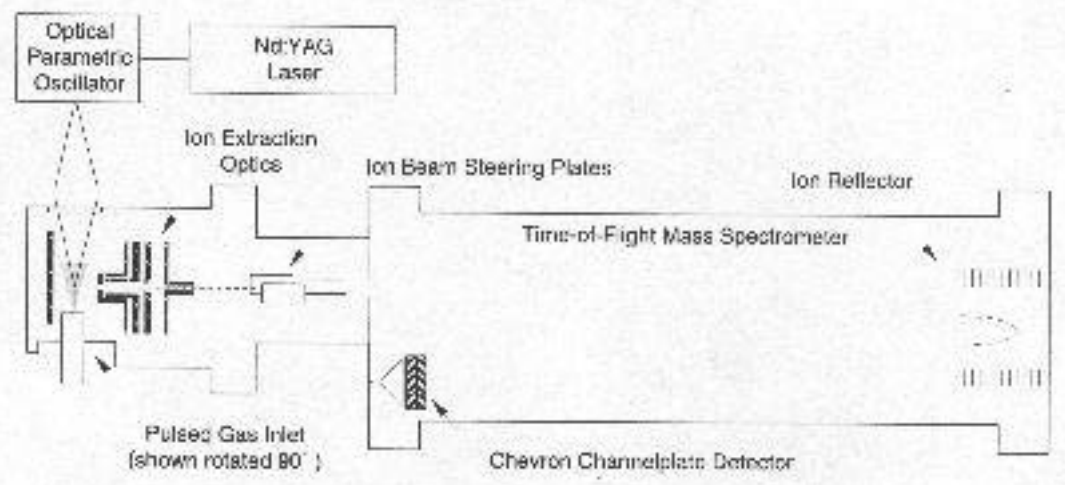

Fie. I. Selcanaty: of the SRI jet-R EMPI appatatus. 
schemes for more than 30 years. SRI firs! uombiretl the use of a pulsed gas inlel with RFMPI as eirly as 198 ; in studies of the ionication of $\mathrm{H}_{2}$ (X) et. al., 1987) and $\mathrm{l}$; (Bischel, 1988), Sinow those exirly stusies, wa bave contimued in uxe and develop pulsed gas valve sourses tor a lange of spentroscopic and detection upplzauons. Pulsed gas values pruyide at aumber of advantages over contimusus gas irlets, including reduied gas tlow and lence stmiller valcutm puraps, tigher local gas densities. ants veli-delized spatial distribution. The major shortcomings of pulsed valves are their teduced throughout and duty cycle as compared with is continuous gas inlet. and their incressed meerarical complex irs. When com bined with a pulsed ionization laver, laweer, the re duced duty cycle of a pulsed vaibe as comorat with is continuous gas inle: is un lenger a factor.

Many mulsod salve desizns tave been develoreal based on simple elecitumestatrutal solenuid mecth:nisms, picznelestic actuaturs, milnedic repuision devices, and modifed fuel anectors. Pulsed ducations range from is fex microseconds to burdreds of microsoennds, while repetition rates reach to to $80 \mathrm{~Hz}$, or higher, defending on the pulse durution anc ralve design. A critical porneneter for the pulsod valve in a dioxin C'EM is the tempersture at whwh it can oncrate. Becuuse of the low volatility of the heavier dioxin congeners. the inlel sampling lines and pulsed valve must be oontinuvusly heated above $250^{\circ} \mathrm{C}$. Although many of the carticr valve desigas could not be heatod mueh above $160 \mathrm{C}$ due to temperuture limits of the scaling materials, designs (Bahut et al., 1987; $\mathrm{Li}$ and Luhman, 1989) now exist tor pulsed valves that can operate routinely a: termperatures above $4 \times / C$.

Resonatity eithanded ntidis-pholon ionzizsoirn is an efficjent and highly selective iorization methes and as such, REMPI is well suited to combination with mas spectroscopy. Fig. 2 is a diagram of RFMIJ. A inolecule

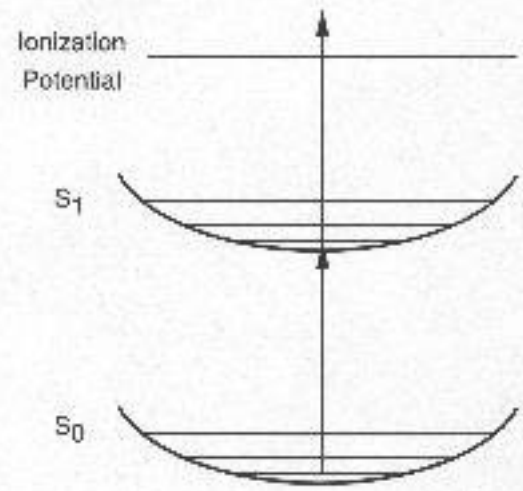

1ig. 2. Diigrim ef the REWPI process. is raised froto the tround siete, Sin to the fist exeited state, $\mathrm{S}_{1}$, by one phown. and suxeczuently ienized $\mathrm{b}_{3}$; serond photor. Although the two-photen RFMll schene. 45 shown in Fiz. 2, is the most common, and by fur most sensitive, botl the excistion and ionizatinn stepe each ean ic peformed with two un noure phutsons. Selectivity is provided by the cesonance of the fort is soption with the exeited state $S$. Lonication frum S: procesds cfficiently, particularly when only twa proters are required.

Many vithonic levels in the exeatod atats san be used for RF:APl. Ite rats:ton strength, Founck-

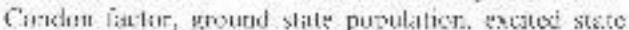
lifelirie, ind tragmenatan thall maly owcur an association with the ionication intledoe chure of the vibrovie levet.

1 urther cont:dereteons for RLMP1 include the us: of vate or tro walvelenglbs icolors; for icnization. Or: color is sompler to imolenent. However, in zerta $\mathrm{n}$ cawez, using a seoond oolor is preterable $n$ essential. For $\mathrm{x} x$ ampls. if the excited state lies lower than half the total ionization energy, a secord photon of the sams wavelength will rot heve eneugh encrge to isnizs the moleeule. For TCDD, the encipy of the $S$ lewel is

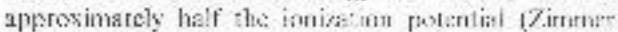
mana et al., 19863. Thus, vingle-colur RFF:MPI is mo-

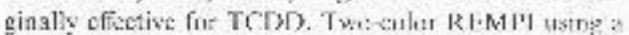
wocend, sighe energy axc hesm misy he a mure effecier! technique for TEDN deketion. The seennd beat ateal net require an additional lase. For exampl:s, when osiog.

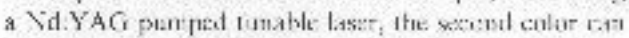
he the fourch hatmonic lipht frum the NalYAG nra duced usirg norlinear crystials

Reaching the first excited slate of ch orinaled dioxins

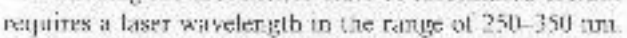
We proilice this wavelerulb cusreatly with a frecuency-

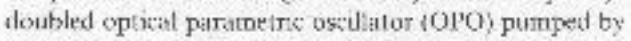
i $\mathrm{Nd}$ Y Y AG taser. $A$ practical system for fir d meisure. meats nught also be bilsed on a tauable solid stite liser suth is Ti:Sipphire or a direct diods-luser-p.Isuped Cr:LiSAL. The barmonics of the Tr:Supphire laser, bowever. have faps and camo: contirucusly cover the wivelength range trom 250 to 350 um.

The mass spectroinetric requitenents of a dioxu CLM justumert aze modest. an upper mass tange of 400 wath unit mass aesoiution. Typical TOF mass zaalyzers can moet these requirenents Lsing a simple, linear tjegt path when combincd with a shert duration. pulsed laser iouization source. Typical operation includes averaging over multiple sears to improve the sign:altc-acise To optinuze this mode of operation. u high repetution rate ionization laser would be desjzable. Acbreving tigh mass resolutiou with a TOF instrument is siritghttorward using a pulsed lase: tor jotuzation becuuse of the arroon spatizl, tempors.. and coergy sp:ead of the nascent iors. 
An increasingly commen $T(1)$ upproth is the use of an ion nirens, cr reflex:tunt-type TOL. Resolving powers ini An of more than luou win be readily obreinod using a reflactron sith a pulsed lase: ionization wura: Al though mas: resolviur powers in excess of inofi are nn nocssary to sepatrale edjecent mass peaks exen at the highest mas in chasge ratios expected ia a diosin CF.M. high reshlt in gres nge to signals with much n.trower. and henoe ruct tigher. peiks. This nereased peak an pilitude feads directly to an increased signa to noise ratje.

A istenalicant improvemen ir resolution and sjenal Jevel can also be obrainos hrough the raretul design of the ion extractiou notick. As a resul of t5 pulsed lese: Jorization sheme, ise fumbetsen ss constraned to occu:

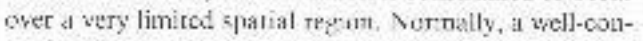
struined ion formation nevion asa be teadaly coupled with aigh efticieney to a rot miss analyzer usiag simpic io a extraction optics. In our instrtment. hoxeves, an additional spatial cunstraint is unposed by the use of a pulsed fas inlat. B.tatuxe this optumun icnization region sil typically be orly it few cercumeters from the exit orific: the ion eptics must be alrefuly designod w inclutiz the inflesenes of the pulsed rilve on the extractina fiskds.

\section{Ixperimutal conditions}

The Jaser syston used in tiese expe-inted cursists of it Cuntunum Poserlite Precisin soll Nd:YAG and Sunbile LX OPO with a noninal turing raure an the vis:bie betweer 415 and 710 nm. a 5 as pulse width. and i repettion rate of $10 \mathrm{H} / \mathrm{l}$ leejuency deubling is actueved using an Triat Autotracker II. The optical lenewidth of the s, tem is approximately $0.1 \mathrm{~cm}^{-1}$.

The pulsed valve is in unmodited General valve series 9 unit. with an urfice daureter of $0.5 \mathrm{~mm}$. The rominal opening time was $190 \mu \mathrm{s}$. with a $2.5 \mathrm{~cm}$ separetion between the exil of the valve and the ionization segion. With the sample reservorr at atrospheric pressurc, the two $250 \mathrm{l} / \mathrm{s}$ turbomokeular pumm (Varian Turbo $\mathrm{V}-250$ ) maicuain pressuzes in the ionization chamber and ma ss spectrouneter regions of I $\times 10^{5}$ ", and $5 \times 10^{2}$ Tor, tespoctively.

The ionization cusctitions used to acquire the data included a pulse energy of approsimatcly $\mathrm{Im} . \mathrm{I}$ in a 1.5 man diameler laser beim. Ion signals from the R.M. Joidan reflectrun $101-\mathrm{MS}$ kere amplified by a Orlet 9306 firearmplifier with a tain of 85 and a $1 \mathrm{GH}_{2}$ handwill1, and recorded by a $500 \mathrm{MH}$, Sigrales D.A500A dipitizes

\section{S. Results}

Thee cungerses huve boen cxaminat in pure vapus ferm. 2-mutuchlorodibenzodioxin. 2,T-dichlotextiben- sxilukin, and 2.8-d:thorosibeazndioxin. RLAPI apos17i for each of these compenals wate been reported

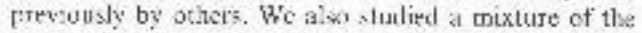
2,- - ichloredibeazodioxin, and 2,8-djchlorodikenzod usin coneceners. This is the firs line that a rixture of twu clesely related dima in eongeners bas been measures USIIE KT-REMPI.

I tegs. 3-5 show the incasured jel-RLMP1 wavelength crpendence tor the the dicxin congeners studicel in puse sapor form. Nole that for each congener, the 203 signal show's rutuerous, barrow, abworption petka corresponding In resomant excitecion of differenl re-vibratienal laveh in lite excited elsetrenic state $S$ of eush

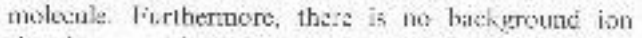
signal measured al ofl-resonano waseleng ls, $I$ or euch wingener, the corzespondang mass spectrom stror's onl? the expected puren: ion peaks, intuding the chlorine isolupic peaks.

of parlictlar intecest and impurtanes ate the datat shuwn in Fig. (1. Here, the simple consisted of an equinokir nixure of the 2,7 and 2.8 -dxtuorodibcnzodivan wongeners. To simplify tbese lests. a small gututuly of the liquid nixlure wils irjected into a staidess sted sample fias, on the upstream side of the pulsad valve, and fuom air was usud us a zearier gai. All components in the sanplestresta were hatod to pecren! concisasatior.

Several ahsmiption peilis corresponding to each of the ongeners liave heren ouled to show that many wavelengths are avalibble lor separutely detocting these two closely relaled congeners in a mixtare. The mixture data in Fie 6 are esserticilly the sum of the waveleagth scans for the se-jarate cumpoucne siowen in Figs. 4 and $S$.

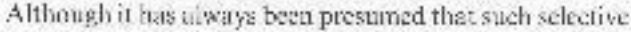
derection of dosely relatod congeners was possible using this method, this is the first ileur demonstration of this selectivily, Of course, other compounds with resonant ahsorplions at wavelengthe coincident with PCDDIF molecules will yield jons with dificring mass-tn-charge ralios, and thus be separabie in the TOF-MS.

For many molocules of interest, narticularly teitachlonuted and higher diozins and furans, the onecolor, two-photen REMPI excitation and ionization x.bejae is not possible as the first excited state lies lesi Itwen bulf way to the ionization limir. Fon those melecules. a two-color REMPI scheme must he used where a turable wavelength is used we eacite $S_{1}$ resorianly, and a second, shorter wareleagth is used to athieve iurization. To demonstrate the two-colut approsech, we dave exstruned the jet-REMPI waveierigt b dependente for 1,2 . dict.orobeazene. The additional lawer pulse for these Jne:lsu rements was generated by doulling, exces $512 \mathrm{~nm}$ secoad harnonic of che Nd:YAG fundamealal. This second wuvelength pulse owerlam the turiatile pulse spillially sithin the ion source: however, it is lemporally delayed by $-5 \mathrm{~ns}$. Altheugh tie delay is acjustitble, $5 \mathrm{~ns}$ 


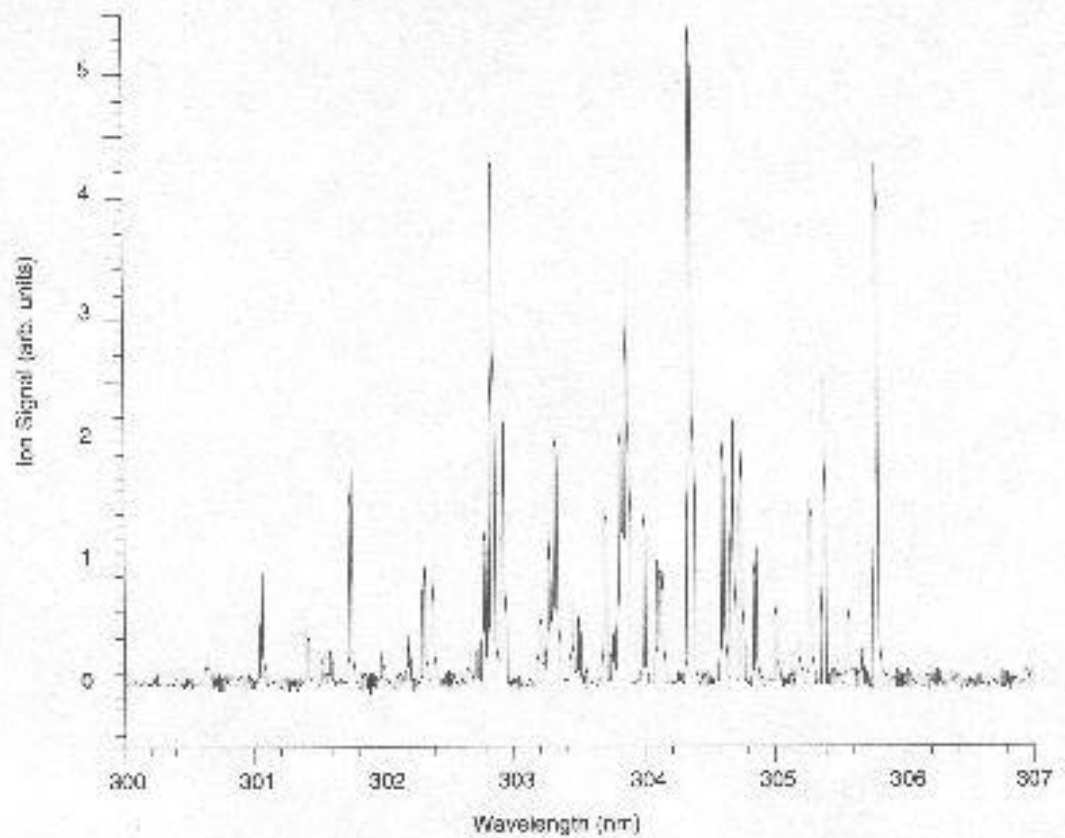

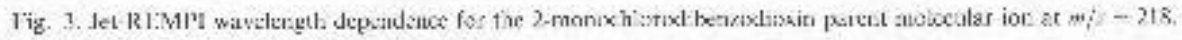

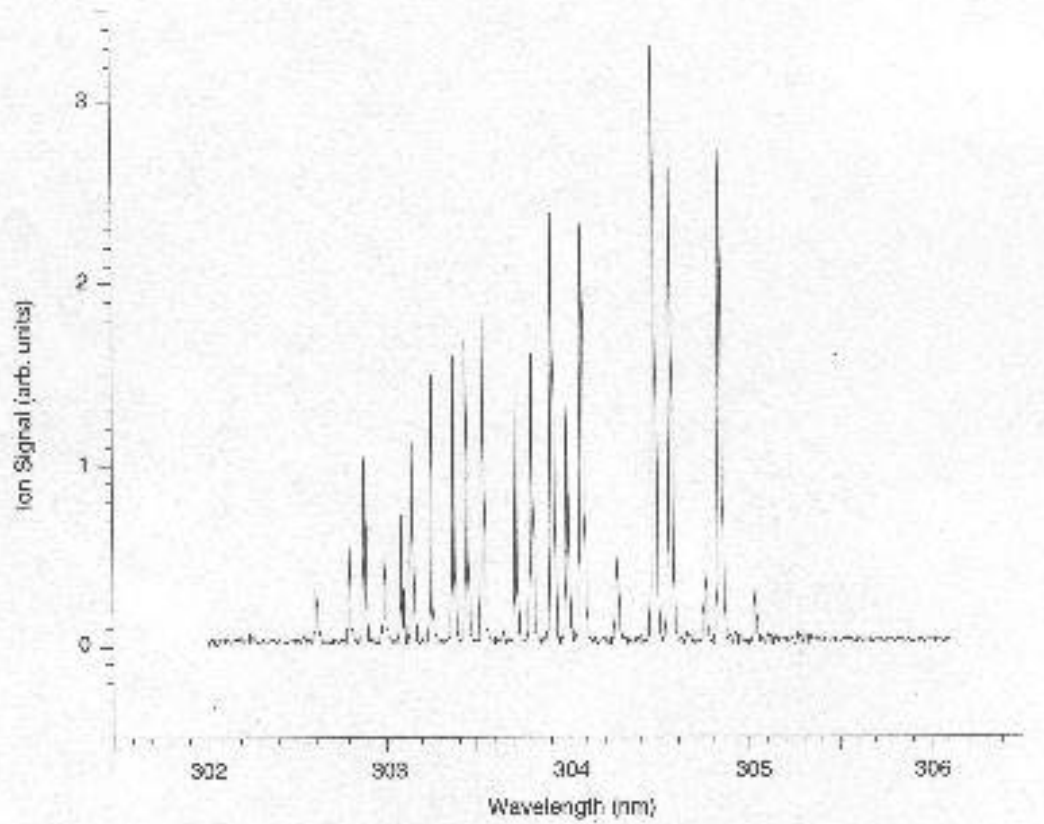

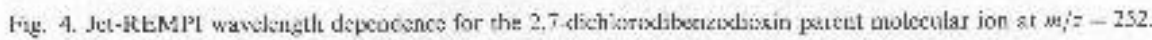




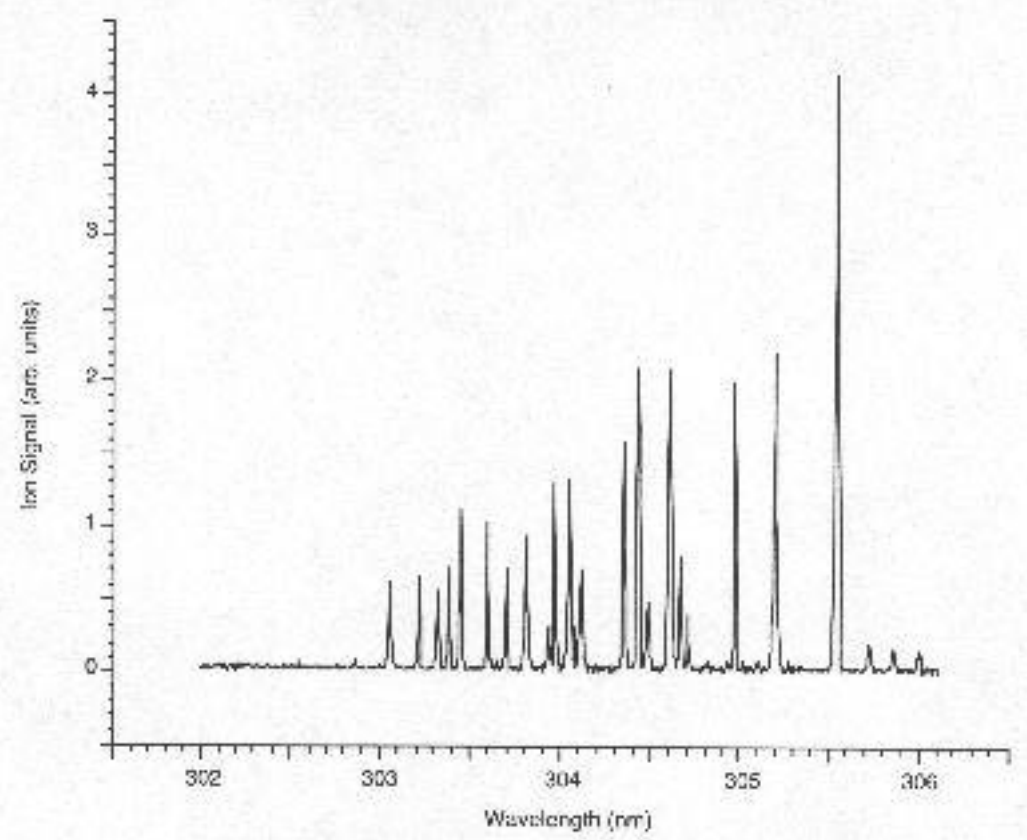

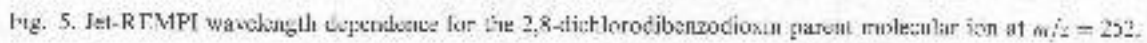

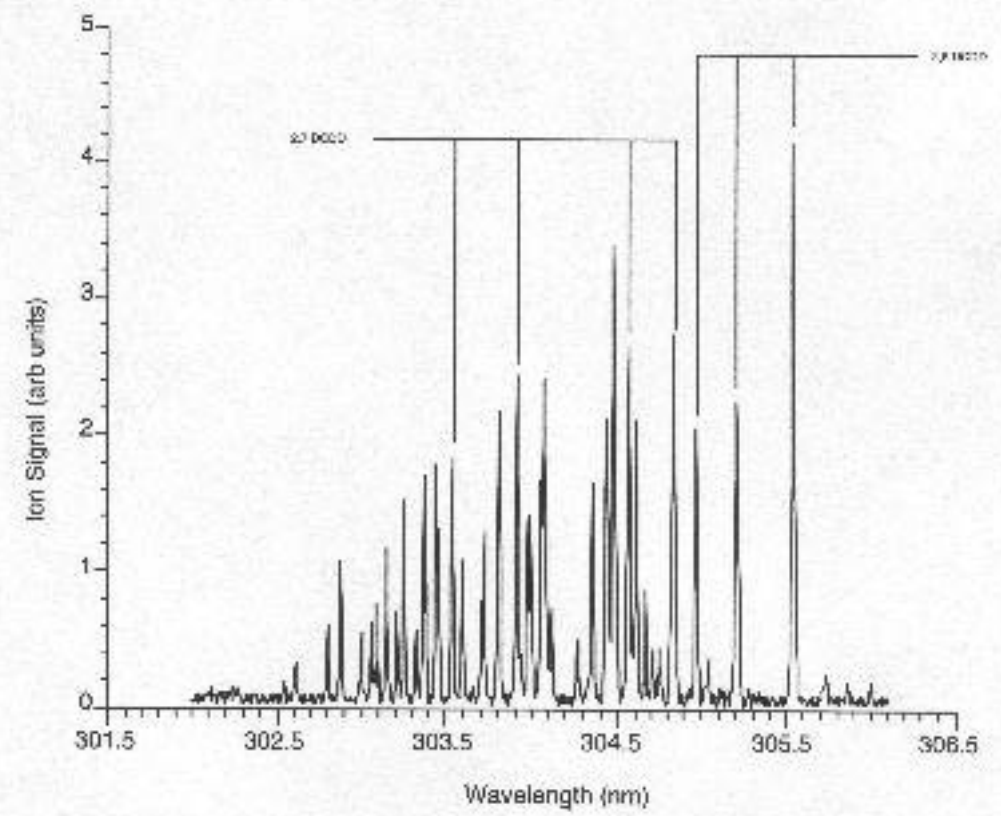

Fig. 6. Jel-kL.t11' watelength dependence for $n$ mixture of 2,7-12.DD and 2,k-DCDD, 


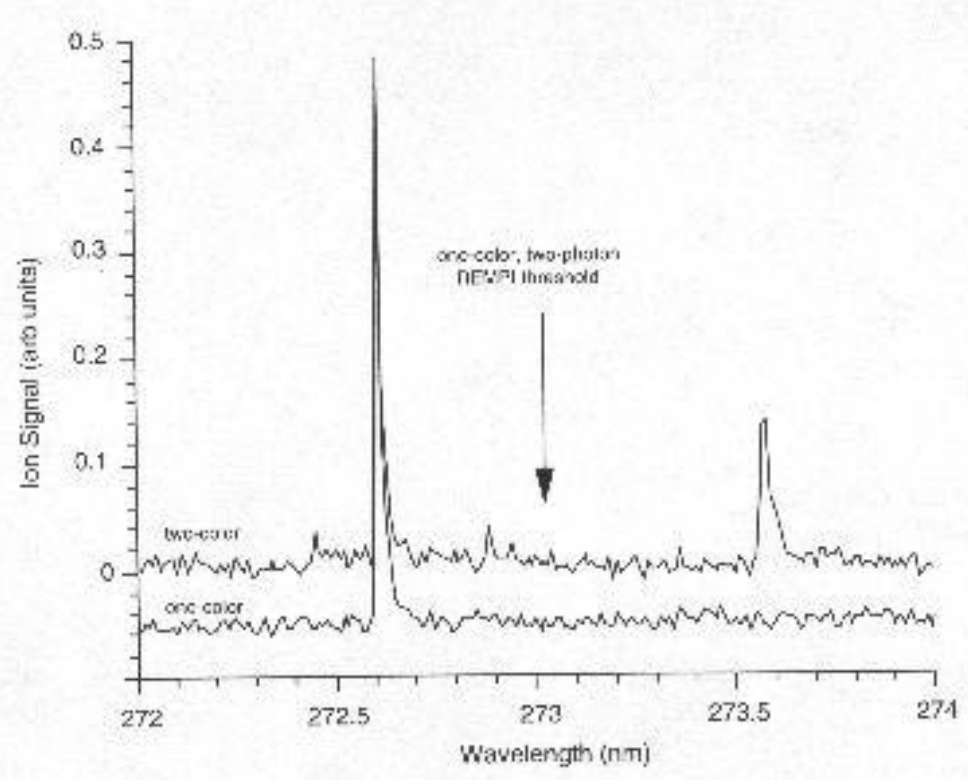

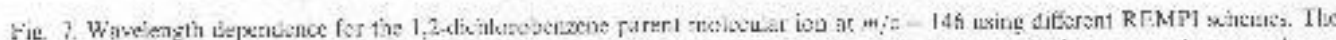

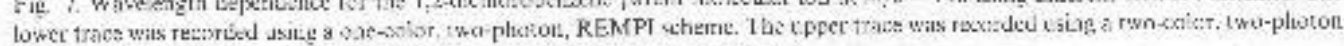

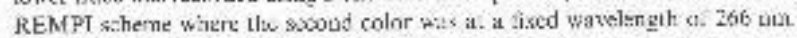

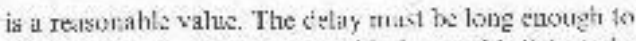
ullow muecules in resonsace with the tunable light to be excited before heing ionizod by the $266 \mathrm{~nm}$ photon, yet not so Jong linat tieg are either lost fruen the iorization region of the molecule returas to tbe yround state.

In Fig. 7. we show the mass-tu-charhe 146 ion signals for 1,2-duthlorobenzene mesurured with onc-color. und two-color excitarion schames. Note that cie large stsorption peak at $272.61 \mathrm{~nm}$ appears using both ionization schemes. However, the absorption peak at 273.58 nm apsears only in the two-color sjgrial. This feature arises from the abworption of one pholun that is resonane with vibrutconal $y$ excited S. moiecules, followed by absorption of it $266 \mathrm{~mm}$ photen to pruduce ionization. Recause the ubsurption of a scoond phuion at $273.58 \mathrm{nt}$ cannoc jonize 1,2-dichlorshenzene, the feature does 100 appear in the one-culat RFMPI spectruen. This observation agress with the expectation that the one-colo: two-photon jonicatiun threshold corresponds w a wivelength of $273.03 \mathrm{~mm}$.

\section{Conclosions}

We have assembled a prototype dioxin CEM hased on the jet-REMP1 approikch. Using this systez, we have dernorstrated detection of several specizic ctuorinatod dioxis cunseners at the pazts-per-trillion concentration renge an kaburatory air. We tave shewe, for the first time. that the jel-REMPI excjtetjun spectrum for it mixture of wo clocely relsted dichlurinated diexin molecules is the stm of the indaridual excitation spectra. This result supports the viability of usiru iet-REMPI as a dioxin CLM for real-work applizations involving mixcures of PCDDif congeners. Also, we have neilsured the first two colns jet-REMPi excilation spectra for a chlorinated uromatic oompound.

\section{Aitinamledgenseuts}

This work wus supported by the Federal Finergy Techassolngs Centes of the Department of Energy under contrat DF-AC26-981\%-40370. One of us $(\mathrm{KC}$ ao bnowledges support from the National Science Foundation, Mathenatics and Pbysics Directorute, Rexearch Lxperience for Lndergraduates Prngram.

\section{References}

Babnt, D., Cheshnorshy, O., Exen, T., Litvie. N., Magen, Y., 1987, Gereration and detection of in kease claster beams. Jotarnil at Physieal Chenistry 51, 24612-2462. 
Beigan Fieuch, N., Gullest, B.K.. Oser, II., Girotiter, H.-H. Natsclabs. D.. J99?. Jet-RES.PI dioxin CE.M test prograru fial tepor1. Westanglwuse Sazrnah River.

Bischel, W.K. 1988. Two plotos cetection Ischniques it atusic fluotite Final Repat for the Air Torte oflice :s Scicutiāe Resanch, SRI Projxt PYC.k121.

Crosles, DR, Cospioia, Mf..1, Tars, Ci.42, Oser, H.. 1999

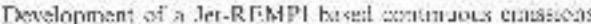
menitor for dioxirs, Ir: Prixedtirys w the Latsuations Conference on lnciaeration ant Tliemas Tisesucnt Tertnobsies. $\mathrm{T} 5 \mathrm{3}$

Gellet, BK, 1997. F.PA Natioral Risi Mansgeucet Latou.

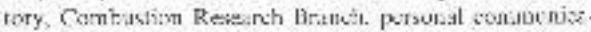
rinr.

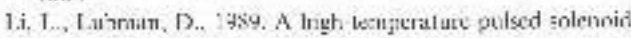

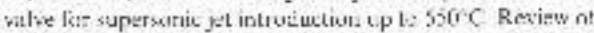
Sciertilik Irstrunents 60. 1Y9 SOI.

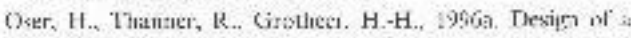
matite bevice basad on jet-KEMPt for the utrasensitin delextson of touse yas exajpeut-ds. Lo: Pisceadings of the

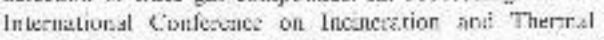
Tratunint leclunitogits. py. 33:-392

Oser, H., Thanner, R.. Gretheer, H.-H., 1996t Jet-RT.MPl cur Lie detcetiol of trax pas compounds in cotnitic gas.

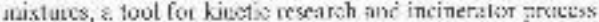
control. Combustion Swince noc Tectnoingy 116. \$67.

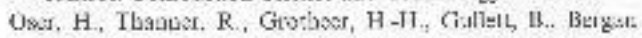
French. N., Nzrschke, D, 1997, Di.R yel RI:NIPI as a

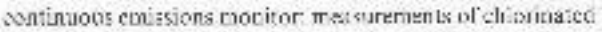
dibenzodioxirs, In Proxedings af the International Cusfererce on Incine arion and Themal Tratursa. I schuolopies
Ose, H., Crostey. I, Cospola, M, Faris, G., 3959. Developnisat of a scal-rione soutiovsus emiszions monitst fo:

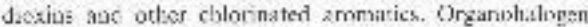
Cutpouts 4 - $5.551-554$

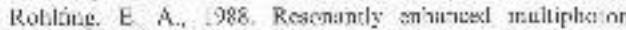

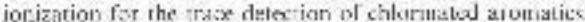
Lo: P-scestires of the 22and international Symegustum or Combustion. The Combustima lakilute, p. 1843.

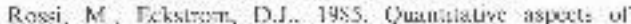

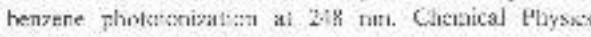
1 Aters 120,115 128.

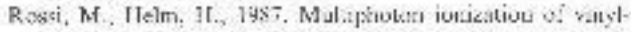

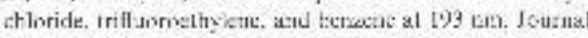

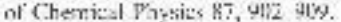

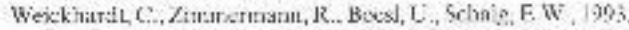

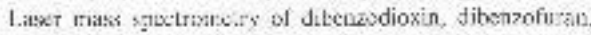

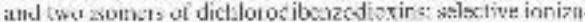

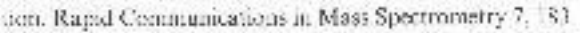

Xu. 1..Y.. Isuzoi. 1., Kaclere, H. Helor, H, 1957 Four-

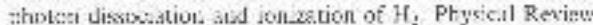
A $36,56-15-5652$.

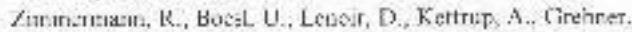
Ta.L. Ne.sssor, H.J. 1995 The isnization eturezex at' fotwde sivated d thenso-p-tioxine new expotismerital naselts atd theoretical studies international Jisurtial of Mass Spectroluatry aud Ion P-iceses 14., 97 2018.

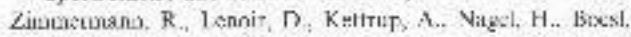

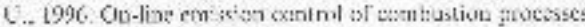

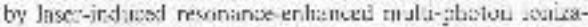

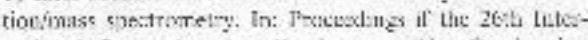

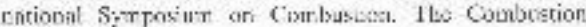
lastiture, p. 2855 


\title{
Draft
}

\begin{abstract}
APPENDIX E
“Development Of A Real-Time Continuous Emissions Monitor For Dioxins And Other Chlorinated Aromatics"
\end{abstract}

Harald Oser, David R. Crosley, Michael J. Coggiola, and Gregory W. Faris

Organohalogen Compounds 40, 551(1999) 


\title{
DEVELOPMENT OF A REAL-TIME CONTINUOUS EMISSIONS MONITOR FOR DIOXINS AND OTHER CHLORINATED AROMATICS
}

\author{
Harald Oser, David R. Crosley, Michael J. Coggiola, and Gregory W. Faris \\ Molecular Physics Laboratory \\ SRI International, Menlo Park, California
}

\section{INTRODUCTION}

Emission control strategies must ultimately rely upon a careful assessment of the link between health effects and ambient, human exposure levels to hazardous air pollutants (HAPs). Because polychlorinated organic compounds in general, and dioxins and furans in particular, are among the most toxic of HAPs, SRI International is developing a continuous emission monitor (CEM) for these species using the Jet-REMPI technique under support from the U.S. Department of Energy (DOE). The unique capability for real-time detection and identification of these and other toxic HAPs directly in incinerator emissions using a CEM instrument is an essential component of emissions modeling, dispersion modeling, source apportionment, and ultimately, of human exposure modeling. JetREMPI is the only instrumental technique that can provide the speed, breadth, and sensitivity of measurement capabilities that are required in support of the development of a comprehensive strategy to monitor and control emissions of dioxins and furans from waste incinerators.

Our technique will provide real-time dioxin and furan concentrations rather than the multi-hour or multiday averages as in the case for existing, conventional sampling and analysis methods. This capability will allow a detailed investigation of the relationship between dioxin emissions and the incinerator operating parameters and waste feed characteristics. Such a study may reveal that "puffs" (transient pollutant emission events) account for a majority of the dioxin emissions, and hence only short-term control methods would be required to meet timeaveraged regulatory emission limits. Such control strategies may be much more cost effective than full-time emission control approaches. Tailoring the emission control strategy in response to minute-by-minute changes in the emission of hazardous pollutants can only be accomplished if a suitable CEM is available, such as our Jet-REMPI based instrument.

\section{MATERIALS AND METHODS}

Our approach to acquiring the dioxin and furan levels is to perform direct measurement of specific congeners using the Jet-REMPI method. Jet-REMPI is an ultra-sensitive analytical technique that can selectively identify and quantify vapor-phase constituents present at ppt levels in incinerator emissions without preconcentration or sample collection.

In recent years, many significant improvements have been made in the detection of hazardous, vapor-phase, organic compounds of environmental importance. Several research groups, including SRI, (1-4) DLR, $(5,6)$ and The Technical University of Munich, Germany, $(7,8)$, have demonstrated instruments or components of instruments based on REMPI and mass spectrometry.

Ions produced by resonance enhanced multiphoton ionization (REMPI) are often detected using a time-offlight mass spectrometer (TOF-MS) that takes advantage of the pulsed nature and well-defined temporal character of laser ionization. The simultaneous detection by mass and wavelength yields extremely high chemical selectivity 
crucial to identifying one trace compound in the midst of many other similar ones. Typical sensitivities of conventional REMPI TOF-MS systems that do not use sample molecule cooling are in the $\mathrm{mg} / \mathrm{dscm}$ range and higher, which is totally insufficient either for a laboratory system or for regulatory monitoring of chlorinated aromatics in a waste treatment process such as an incinerator.

A major improvement in sensitivity without loss in selectivity can be achieved using a pulsed gas valve that produces a supersonic expansion. Pulsed gas valves provide a number of advantages over continuous gas inlets, including reduced gas flow and hence smaller vacuum pumps, higher local gas densities, well-defined spatial distribution, significantly reduced translational energy distribution orthogonal to the propagation direction, and reduced internal (vibrational and rotational) temperatures leading to greatly enhanced spectroscopic resolution.

REMPI is a highly sensitive, highly species-selective, gas-phase analysis technique that has been applied to numerous problems in molecular spectroscopy and combustion research. For REMPI of complex molecules, such as dioxins, the spectra can be simplified greatly by expansion through a nozzle. Adiabatic expansion results in low sample temperatures, which increases the electronic ground state population and narrows the resonance line widths through reduction in molecule velocities (reduced Doppler or temperature broadening) and through reduction in transition-perturbing collisions (reduced pressure broadening). These reduced linewidths lessen the ionization of other molecular species (interferences) - leading to improved selectivity - and make the peak absorption larger leading to improved sensitivity. One or more lasers are used to ionize the cooled gas molecules by absorption of two or more photons, one of which must be resonant with an electronic transition in the target molecule.

Because different isomers of a given chemical composition may have very different toxicities, it is essential that a measurement instrument be capable of distinguishing among isomers. Our instrument is able to do this without any sample pre-separation, such as by gas chromatography, for example, because of the inherent ability of optical spectroscopy (REMPI in this case) to readily distinguish among isomers. The selectivity of the optical spectroscopy is vastly improved through the pulsed nozzle cooling effects, as discussed above.

The mass spectrometric capabilities of our system include an upper mass range typically up to $500 \mathrm{amu}$, limited only by the size of the mass spectral data files. Typical laser-based TOF mass analyzers use a simple, linear flight path combined with a short duration, pulsed laser, ionization source. Low signal levels that may result from the use of a very short ion formation pulse can be compensated for by signal averaging using multiple scans. To optimize this mode of operation, a high repetition rate ionization laser is used. Achieving good mass resolution $(\mathrm{m} / \Delta \mathrm{m})$ with a TOF instrument is straightforward using a pulsed laser for ionization because of the narrow spatial, temporal, and energy spread of the nascent ions.

\section{RESULTS AND DISCUSSION}

The laser system used in our preliminary experiments described below consists of a Continuum Powerlite Precision $9010 \mathrm{Nd}$ :YAG and Sunlite EX OPO with a nominal tuning range in the visible between 445 and $710 \mathrm{~nm}$, a $5 \mathrm{~ns}$ pulse width, and a repetition rate of $10 \mathrm{~Hz}$. Frequency doubling is achieved using an Inrad autotracker II. The optical linewidth of the system is approximately $0.1 \mathrm{~cm}^{-1}$.

The pulsed valve is an unmodified General Valve series 9 unit, with an orifice diameter of $0.5 \mathrm{~mm}$. The nominal opening time was $150 \mu \mathrm{s}$, with a $2.5 \mathrm{~cm}$ separation between the exit of the valve and the ionization region. With the sample reservoir at atmospheric pressure, the two $250 \mathrm{l} / \mathrm{s}$ turbomolecular pumps (Varian Turbo V-250) maintain pressures in the ionization chamber and mass spectrometer regions of $10^{-5}$ Torr, and $5 \times 10^{-7}$ Torr, respectively.

The ionization conditions used to acquire the preliminary data included a pulse energy of approximately $1 \mathrm{~mJ}$ in a $1.5 \mathrm{~mm}$ diameter laser beam. Ion signals from the R. M. Jordan angular reflectron TOF-MS were amplified 
by a Comlinear preamplifier with a gain of 10 and a $350 \mathrm{MHz}$ bandwidth. Signals were recorded by a $500 \mathrm{MHz}$, Signatec DA500A digitizer.

A sample of 1,2-dichlorobenzene was used for the initial instrument testing. To simplify these tests, a small quantity of liquid was injected into a stainless steel tube on the upstream side of the pulsed valve, and room air was used as a carrier gas. All components were at room temperature. 1,2-Dichlorobenzene is commonly used for testing because of its high vapor pressure at room temperature, and it may also be an important indicator species of total dioxin levels (9). Moreover, the REMPI excitation spectrum is well known, so that tuning the laser to resonance line at $261.2 \mathrm{~nm}$ produced the mass spectrum shown in Figure 1.

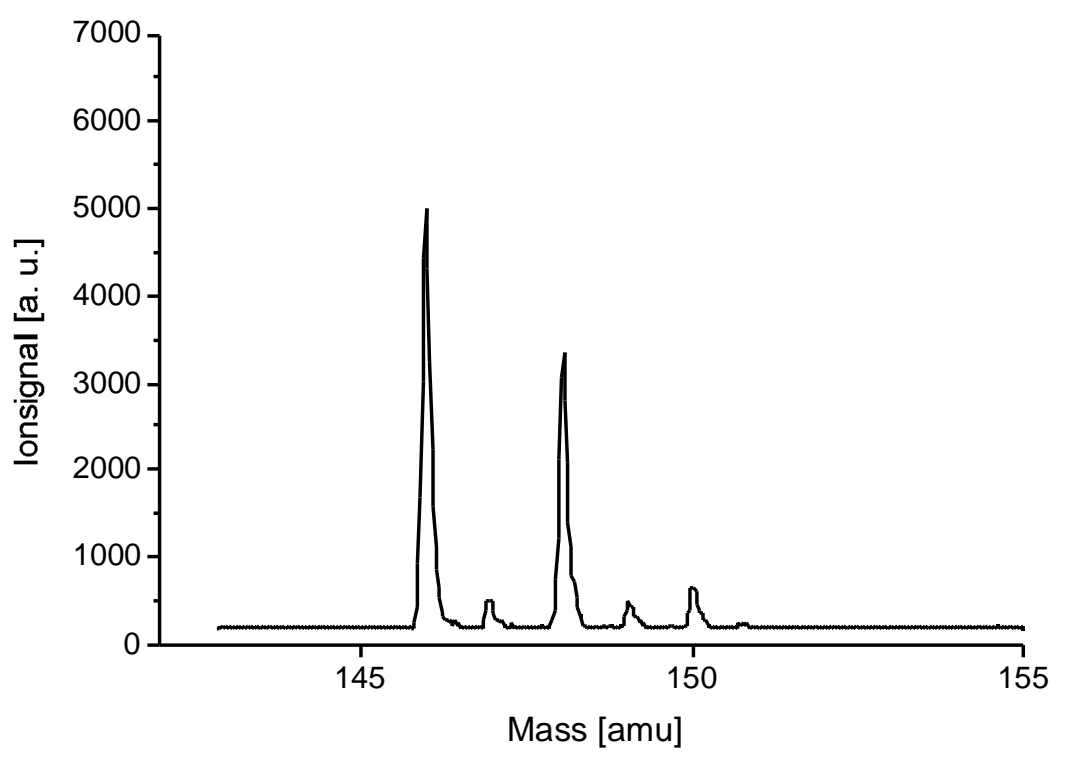

Figure 1. Jet-REMPI mass spectrum of 1,2-dichlorobenzene at a wavelength of $261.2 \mathrm{~nm}$.

We also performed a rapid survey of the m/z 146 ion signal strength as a function of laser wavelength to produce the REMPI excitation spectrum. That spectrum, shown in Figure 2, is in good agreement with previously reported results for the same molecule. Both the resonance locations and their peak heights are consistent with published REMPI spectra, indicating that our instrument is functioning correctly. 


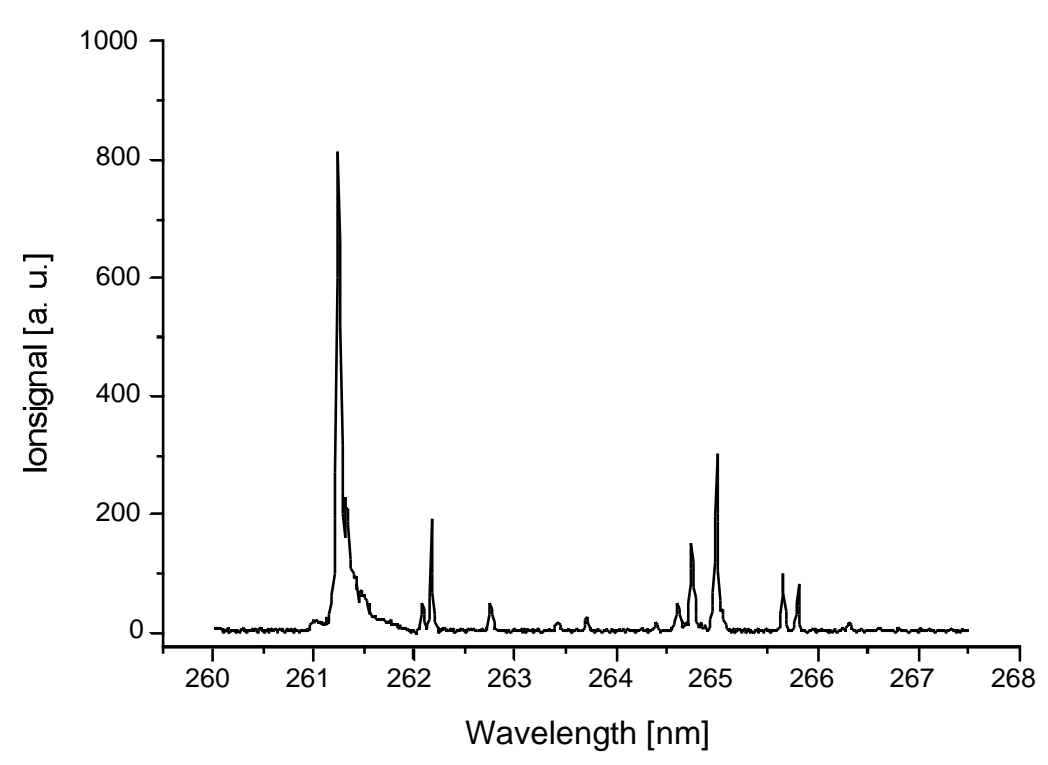

Figure 2. Wavelength dependence of the m/z 146 ion signal for 1,2-dichlorobenzene.

Acknowledgments

This work was support by the U. S. Department of Energy, Morgantown Energy Technology Center, Morgantown, West Virginia under contract DE-AC26-98FT-40370.

\section{References}

1. Bischel, W. K., "Two Photon Detection Techniques for Atomic Fluorine," Final Report for the Air Force Office of Scientific Research, SRI Project PYC-8320, June, 1988.

2. Xu, E. Y., Tsuboi, T., Kachru, R., and Helm, H., Phys Rev. 1987, A36, 5645.

3. Rossi M. and Eckstrom, D. J., Chem. Phys. Lett. 1985, 120, 118.

4. Rossi, M. and Helm, H., J. Chem. Phys. 1987, 87, 902.

5. Oser, H., Thanner, R., Grotheer, H. H., Gullett, B., Bergan French, N., Natschke, D., Proceedings, 1997 Int. Conf. On Incineration and Thermal Treatment Technologies.

6. Oser, H., Thanner, R., Grotheer, H. H., Proceedings, 1996 Int. Conf. On Incineration and Thermal Treatment Technologies, pp. 387-392.

7. Weickhardt, C., Zimmermann, R., Boesl, U., Schalg, E. W., Rapid Comm. Mass Spectrom. 1993, 7, 183.

8. Zimmermann, R., Boesl, U., Lenoir, D., Kettrup, A., Grebner, Th. L., and Neusser, H. J., Int. J. Mass Spectr. and Ion Phys. 1995, 145, 97.

1. Blumenstock, M, Zimmermann, R., Lehnardt, R., Schramm, K.-W., Kaune, A., Nikolai, U., Lenoir, D., Kettrup, A., Organohalogen Compounds 1998, 36, 47-52. 


\begin{abstract}
APPENDIX F
“Development Of A Real-Time Continuous Emissions Monitor For Hazardous Aromatic Air Pollutants"
\end{abstract}
Harald Oser, Michael J. Coggiola, Gregory W. Faris, Bengt Volquardsen, and David R. Crosley

Organohalogen Compounds 45, 141 (2000) 


\title{
DEVELOPMENT OF A REAL-TIME CONTINUOUS EMISSIONS MONITOR FOR HAZARDOUS AROMATIC AIR POLLUTANTS
}

\author{
Harald Oser, Michael J. Coggiola, Gregory W. Faris, Bengt Volquardsen*, and David R. Crosley \\ Molecular Physics Laboratory \\ SRI International, 333 Ravenswood Ave., Menlo Park, California, USA \\ *Fachhochschule Aachen, 52066 Aachen, Germany
}

\section{Introduction}

Under the 1990 Clean Air Act (CAA) Amendments, the U.S. Environmental Protection Agency (EPA) was mandated to implement a research program providing the scientific foundation to develop a comprehensive national strategy. This strategy aim to control $90 \%$, or more of the emissions of the 30 most hazardous toxic pollutants in urban areas. This control strategy must ultimately rely on a careful assessment of the link between health effects and ambient, human exposure levels to hazardous air pollutants (HAPs). Despite the need for such data, reliable ambient concentrations have been measured for fewer than $40 \%$ of the 189 HAPs. $^{1}$ Even these limited measurements provide no spatial or temporal information on HAPs levels, whose variation is inherent due to the sporadic, episodic nature of many combustion-related HAPs releases. This information cannot reliably be estimated or inferred by typical current method, like GC-MS. It must be acquired through actual field measurements under typical, timevarying, ambient human exposure conditions.

A powerful alternative to current HAPs monitoring methods is Jet-REMPI-ToF-MS. It combines resonance enhanced multiphoton ionization (REMPI), supersonic jet cooling, and time-of-flight mass spectrometry.

SRI International is developing a continuous emission monitor (CEM) for HAPs using the REMPI technique under support from the U. S. Department of Energy (DoE) and the U.S. Environmental Protection Agency (EPA).

Our instrument will provide real-time HAPs at ambient and toxicological concentrations, instead of the multi-hour or multi-day averages obtained using existing, conventional sampling and analysis methods. This instrument will provide the speed, breadth, and sensitivity of measurement capabilities required by EPA in support of its development of a comprehensive national strategy to control emissions of HAPs from urban sources.

\section{Materials and Methods}

Our approach to detecting toxic organic HAPs and HAP mixtures in urban areas at the required levels is to perform direct measurement of specific isomers using the Jet-REMPI method. Jet-REMPI is an ultra-sensitive analytical technique that can selectively identify and quantify vapor-phase constituents at parts-per-trillion levels or lower.

In recent years, many significant improvements have been made in the detection of hazardous, vapor-phase, organic compounds of environmental importance using Jet-REMPI. It has been demonstrated by several research groups, including $\mathrm{SRI}^{2-5}, \mathrm{DLR}^{6,7}$, and The Technical University of Munich, Germany ${ }^{8,9}$ - under the condition of supersonic cooling, the two-dimensional detection scheme based on laser excitation spectrum plus mass spectra can provide a unique method of molecular identification and structural detection. 
In the REMPI process, one or two laser wavelengths are used to ionize gas molecules by absorption of two photons, one of which must be resonant with an electronic transition in the target molecule. This optical resonance with the molecular quantum state provides part of the selectivity for Jet-REMPI.

Ions produced by REMPI are detected using a time-of-flight mass spectrometer (TOF-MS) that takes advantage of the pulsed nature and well-defined temporal character of laser ionization. The simultaneous detection by mass and wavelength yields the extremely high chemical selectivity crucial to identifying one trace compound in the midst of many other similar ones.

For REMPI of complex molecules, such as HAPs and even dioxins, the spectrum can simplified dramatically by expansion of the sample through a narrow orifice. The supersonic cooling step results in low sample temperatures, increasing the electronic ground state population and narrowing the resonance line widths through reduction in molecular velocities and through reduction in transition-perturbing collisions. These reduced linewidths are eliminate the ionization of other molecular species (interferences) - leading to improved selectivity - and make the peak absorption larger - leading to improved sensitivity.

The pulsed gas valves also provide advantages over continuous gas inlets, such as reduced gas flow and hence smaller vacuum pumps and is compatible with the pulsed nature of REMPI.

Because different isomers of a given chemical composition may have very different toxicities, it is essential that a measurement instrument be capable of distinguishing among isomers. Our instrument does this without any sample pre-separation, such as by gas chromatography, for example, because of the inherent ability of the laser excitation step to readily distinguish among isomers.

The mass spectrometric capabilities of our system include an upper mass range typically up to $500 \mathrm{amu}$, limited only by the size of the mass spectral data files. The TOF mass analyzers uses a simple, linear flight path compatible with the short pulsed laser. Achieving good mass resolution $(\mathrm{m} / \Delta \mathrm{m})$ with a TOF instrument is straightforward using a pulsed laser for ionization because of the narrow spatial, temporal, and energy spread of the nascent ions.

A more detailed description of our laboratory Jet-REMPI ToF-MS instrument is given elsewhere ${ }^{10}$. Therefore we present here just a brief summary of our instrument.

The sample is introduced into the ionization chamber through a pulsed valve (General Valve series 9) that delivers $150 \mu$ s sample pulses at a repetition rate of $10 \mathrm{~Hz}$. Residual gas pressures of $10^{-5}$ Torr in the ionization chamber and $10^{-7}$ Torr in the mass spectrometer are achieved by the combination of sufficiently large pumps (Varian V-250 and Seiko Seki 301), the short gas injection time, a $0.5 \mathrm{~mm}$ orifice of the pulsed valve, and gas samples at atmospheric pressure.

The laser system consists of a Continuum Powerlite Precision 9010 Nd:YAG and Sunlite EX OPO including frequency doubling with a nominal tuning range in the UV between 225 and $400 \mathrm{~nm}$, a $5 \mathrm{~ns}$ pulse width, and a repetition rate of $10 \mathrm{~Hz}$. The optical linewidth of the system is approximately $0.2 \mathrm{~cm}^{-1}$.

An unfocused laser beam is used, with a beam area of about $2 \mathrm{~mm}^{2}$ and pulse energies of approximately 1 mJ. Ions were mass analyzed with a reflectron type ToF-MS (R. M. Jordan), amplified by an Ortec 9306 preamplifier (gain $=85$, bandwith $=1 \mathrm{GHz}$ ) and recorded by a $500 \mathrm{MHz}$ digitizer (Signatec DA500A).

\section{Results and Discussion}




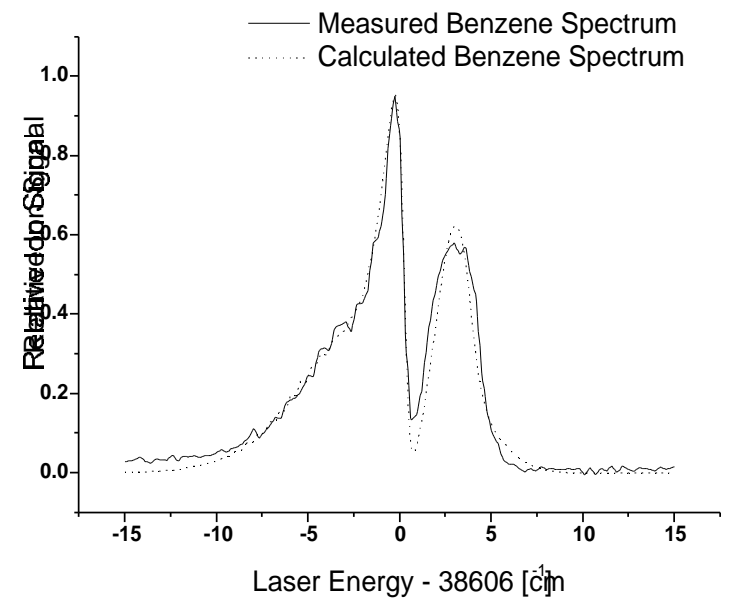

Fig. 1: Relative ion signal and spectroscopic fit for the benzene transition around $259 \mathrm{~nm}$

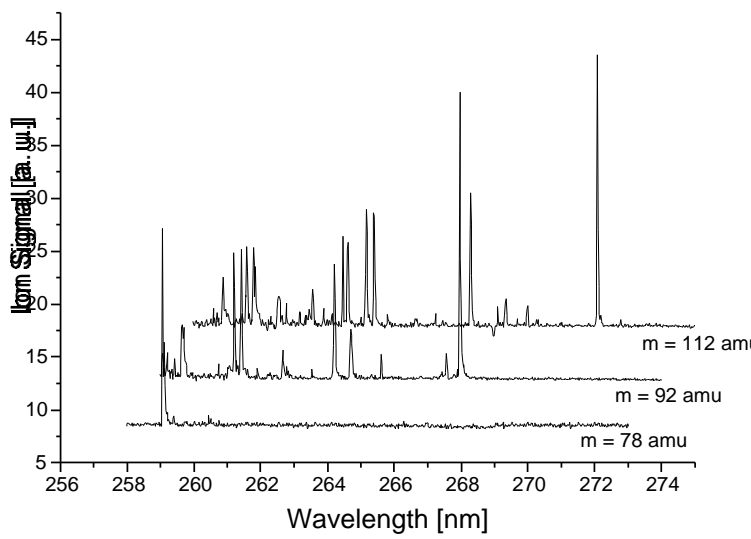

Fig. 2: Wavelength dependent REMPI spectra for Benzene (78 amu), Toluene (92 amu), and Chlorobenzene (112)
The effective temperature of samples in the supersonic jet is important for sensitivity and selectivity using Jet-REMPI. Lower temperatures lead to narrower lines, which improves

selectivity. In addition, there are fewer total lines, again improving both sensitivity and selectivity. To learn the operating conditions that achieve the lowest temperature in combination with the highest sensitivity from our pulsed nozzle/ion extraction arrange-ment, we have used benzene as a thermometric species. The spectroscopy of benzene is known quite well, allowing determination of the effective rotational temperature from measured spectra. Measurements of a single band are shown in Fig. 1. This spectrum was taken at a distance of about $2.7 \mathrm{~cm}$ from the nozzle. The band shown is part of the lowest singlet-singlet transition $\left(\mathrm{S}_{1} \leftarrow\right.$ $\mathrm{S}_{0}$ ) of benzene near $259 \mathrm{~nm}$. The band shown corresponds to many overlapping rotational lines. From the overall contour of these overlapping lines, we can determine an effective rotational temperature of the benzene. We simulate the form of the main contour as a function of temperature. Rotational band calculation follow the method given by Callomon, et al. ${ }^{11}$ The result is also shown in Fig 1. The best agreement of the general shape for the simulated band contour with the experimental result is given at a temperature of $19 \pm 1{ }^{\circ} \mathrm{K}$. This indicates a high degree of internal relaxation. To demonstrate the two dimensional detection scheme based on wavelength and mass we used a mixture of benzene, toluene, and clorobenzene as test substances. These molecules are known HAPs and their REMPI spectra are defined $^{12,13}$. Due to soft ionization, only the parent peaks at their respective masses are recorded and no cross sensitivities occur. The results in Fig. 2 show that we have good optical resolution for our measured spectra. This also indicate sufficient cooling. 


\section{Acknowledgments}

This work was support by the U. S. Department of Energy, Morgantown Energy Technology Center, Morgantown, West Virginia under contract DE-AC26-98FT-40370, and U. S. Environmental Protection Agency under grant R82792701.

\section{References}

1. Koshland, C. P. (1996),'Impacts and Controls of Air Toxics from Combustion," $26^{\text {th }}$ Symposium (Int.) on Combustion, p. 2049.

2. Bischel, W. K. (1988), "Two Photon Detection Techniques for Atomic Fluorine," Final Report for the Air Force Office of Scientific Research, SRI Project PYC-8320.

3. Xu, E. Y., Tsuboi, T., Kachru, R., and Helm, H. (1987), Phys Rev., A36, 5645.

4. Rossi M. and Eckstrom, D. J. (1985), Chem. Phys. Lett., 120, 118.

5. Rossi, M. and Helm, H. (1987), J. Chem. Phys., 87, 902.

6. Oser, H., Thanner, R., Grotheer, H. H., Gullett, B., Bergan French, N., Natschke, D. (1997), Proceedings Int. Conf. On Incineration and Thermal Treatment Technologies.

7. Oser, H., Thanner, R., Grotheer, H. H. (1996), Proceedings Int. Conf. On Incineration and Thermal Treatment Technologies, pp. 387-392.

8. Weickhardt, C., Zimmermann, R., Boesl, U., Schalg, E. W. (1993), Rapid Comm. Mass Spectrom. 7, 183.

9. Zimmermann, R., Boesl, U., Lenoir, D., Kettrup, A., Grebner, Th. L., and Neusser, H. J. (1995), Int. J. Mass Spectr. and Ion Phys., 145, 97.

10. Oser, H., Copic, K., Coggiola, M. J., Faris, G. W., and Crosley, D. R. (2000), "Congenere-Specific Detection of Dioxins Using Jet-REMPI," Chemosphere, to be published.

11. Callomon, J. H., Dunn, T. M., and Mills, I. M. (1966),"Rotational Analysis of the 2600_ Absorption System of Benzene." Philoso. Trans. R. Soc. London Ser. A 259, 499-532.

12. Williams, B. A., Tanada, T. N., and Cool, T. A. (1992), "Resonance Ionization Detection Limits for Hazardous Emissions," 24 $4^{\text {th }}$ Symposium (Int.) on Combustion, p. 1887.

13. Sin, C. H., Tembreull, R., Lubman, D. M. (1984), "Resonant Two-photon Ionization spectroscopy in Supersonic Beams for Discrimination of Disubstituted Benzenes in Mass Spectrometry," Anal. Chem. 56, 1227. 


\title{
Draft
}

\begin{abstract}
APPENDIX G
“Dioxin And Furan Laboratory Measurements Using Jet-REMPI"

Harald Oser, Michael J. Coggiola, Steve E. Young, Gregory W. Faris, and David R. Crosley
\end{abstract}

Organohalogen Compounds (2001) 


\section{DIOXIN AND FURAN LABORATORY MEASUREMENTS USING JET-REMPI}

Harald Oser, Michael J. Coggiola, Steve E. Young, Gregory W. Faris, and David R. Crosley

SRI International, Menlo Park, California 94025 USA

\section{Introduction}

On-line monitoring of stack emissions to determine TEQ values requires at the minimum, sensitivities at the ppq level, which is far below the detection limit of any current continuous emission monitor. An alternative approach is the real-time measurement of indicator substances, like lowly chlorinated DD/DF isomers, by jet-cooled, resonantly enhanced multi-photon ionization (Jet-REMPI).

The use of Jet-REMPI as an indirect real time TEQ monitor requires identification of sufficiently abundant lowly chlorinated isomers that correlate well with the TEQ values. Recent statistical analysis studies of TEQ values and concentrations for a subset of the mono- to tri-chlorinated $\mathrm{PCDD} / \mathrm{F}$ congeners show promising correlations, suggesting that these compounds can act as TEQ indicators ${ }^{1,2}$. The on-line nature of Jet-REMPI provides also a valuable tool for the mechanistic understanding of PCDD/F formation. This understanding may support finding process control methods to reduce or even prevent their formation.

Under support from the U. S. Department of Energy (DoE) and the U. S. Environmental Protection Agency (EPA), SRI International has developed a continuous emission monitor (CEM) for PCDD/F detection using the Jet-REMPI method. In an initial step, the focus of the research was to measure Jet-REMPI spectra for lowly chlorinated PCDD/F isomers, investigate mixtures of these compounds, and apply a more advanced ionization scheme, namely the two color, two photon ionization scheme, to increase the number of REMPI accessible PCDD/F isomers.

\section{Materials and Methods}

Jet-REMPI is an ultra-sensitive, highly selective, analytical technique that can identify and quantify vapor-phase constituents at parts-per-trillion levels. In recent years successful applications in on-line monitoring of hazardous air pollutants produced in waste incineration have been reported by two research groups ${ }^{3,4}$, and many significant improvements have been made in the detection of hazardous, vapor-phase, organic compounds of environmental importance using Jet-REMPI. 
In the REMPI process, one or two laser wavelengths are used to ionize gas molecules by absorption of two photons, one of which must be resonant with an electronic transition in the target molecule. This optical resonance with the molecular quantum state provides part of the selectivity for Jet-REMPI. Ions produced by REMPI are detected using a time-of-flight mass spectrometer (TOF-MS) that takes advantage of the pulsed nature and well-defined temporal character of laser ionization. The simultaneous detection by mass and wavelength yields the extremely high chemical selectivity crucial to identifying one trace compound in the midst of many other similar ones.

For REMPI of complex molecules, such as dioxins, the spectrum can be simplified dramatically by expansion of the sample through a narrow orifice. The supersonic cooling step results in low sample temperatures, increasing the electronic ground state population and narrowing the resonance line widths through reduction in molecular velocities and through reduction in transition-perturbing collisions. These reduced linewidths eliminate the ionization of other molecular species (interferences) - leading to improved selectivity - and make the peak absorption larger - leading to improved sensitivity.

A more detailed description of our laboratory Jet-REMPI TOF-MS instrument is given elsewhere ${ }^{5}$. Therefore just a brief summary of is presented here. The sample is introduced into the ionization chamber through a pulsed valve (General Valve series 9) that delivers $150 \mu \mathrm{s}$ sample pulses at a repetition rate of $10 \mathrm{~Hz}$, which results in the advantage of a reduced gas flow and hence smaller vacuum pumps and is compatible with the pulsed nature of REMPI. Residual gas pressures of $10^{-5}$ Torr in the ionization chamber and $10^{-7}$ Torr in the mass spectrometer are achieved by the combination of sufficiently large pumps (Varian V-550 and Seiko Seki 301), the short gas injection time, a $0.5 \mathrm{~mm}$ orifice of the pulsed valve, and gas samples at atmospheric pressure. The laser system consists of a Continuum Powerlite Precision 9010 Nd:YAG and Sunlite EX OPO including frequency doubling with a nominal tuning range in the UV between 225 and $400 \mathrm{~nm}$, a $5 \mathrm{~ns}$ pulse width, and a repetition rate of $10 \mathrm{~Hz}$. The optical linewidth of the system is approximately $0.1 \mathrm{~cm}^{-1}$. For the two color excitation scheme a combination of tunable UV from the OPO and $266 \mathrm{~nm}$ produced by the quadrupling of the fundamental wavelength of the Nd:YAG pump laser was used. The two laser beams were unfocused, with a beam area of about $2 \mathrm{~mm}^{2}$ and pulse energies of approximately $1 \mathrm{~mJ}$ for the OPO output and $0.1 \mathrm{~mJ}$ of the $4^{\text {th }}$ harmonic were used. Ions were mass analyzed by a reflectron type TOF-MS (R. M. Jordan) with a mass resolution of 1000, amplified by an Ortec 9306 preamplifier (gain $=85$, bandwith $=1$ $\mathrm{GHz}$ ) and recorded by a $500 \mathrm{MHz}$ digitizer (Signatec DA500A).

\section{Results and Discussion}


The on-line detection of lowly chlorinated DD/DF isomers is of particular interest for investigation of the formation chemistry of toxic PCDD/F, and indirect determination of TEQ values $^{1,2}$. For this purpose, we investigate the Jet-REMPI spectroscopy of 2,7- and 2,8-DCDD and 2-MCDF which are proposed as potential TEQ-indicators.

Despite the fact that Jet-REMPI for the two DCDD is already known ${ }^{6}$, it has not been proven yet if Jet-REMPI is a valuable tool to discriminate these isomers. Therefore we conducted experiments by using a sample consisting of a mixture of equal amounts of 2,7- DCDD and 2,8DCDD, desolved in acetone. To simplify these tests, a small quantity of the liquid mixture was injected into a stainless steel sample flask on the upstream side of the pulsed valve, and room air was used as a carrier gas to simulate semi realistic conditions. We estimate that the concentration of each isomer in the sampled gas stream was approximately $1 \mathrm{ppb}$. All components in the sample stream, including the nozzle, were heated to $150^{\circ} \mathrm{C}$ to prevent condensation. Figure 1 shows the ion signal recorded at mass 252 , corresponding to the parent ion of both isomers, as a function of wavelength. Several

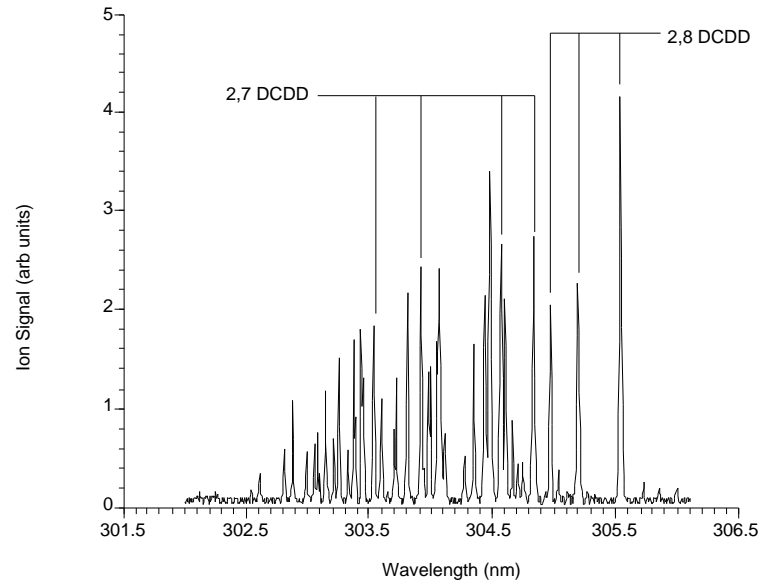

Figure 1: Wavelength dependence of the $\mathrm{m} / \mathrm{z}$ 252 ion individual absorption features are labeled to show which isomer gave rise to the ion signal. Assignment of these absorption features was made by measuring the JetREMPI spectra for each isomer separately and by comparison with the results of Weickardt et al. ${ }^{6}$. From an estimated signal-to-noise ratio of 20 , we calculated a current detection limit of $50 \mathrm{ppt}$ for both DCDD isomers.

The wavelength dependence for 2-MCDF, which has never been examined by JetREMPI, is shown in Figure 2. The wavelength dependence shows a number of clearly resolved peaks, however, the overall ion signal strength was less than for similar dioxin molecules. One common cause of low ionization probability occurs when the two photons do not excite the neutral molecule appreciably above its ionization threshold. One major consideration for improving REMPI sensitivity is the use of a different wavelength (color) for the ionization step, although one color for both the excitation and ionization steps is certainly simpler to implement. 


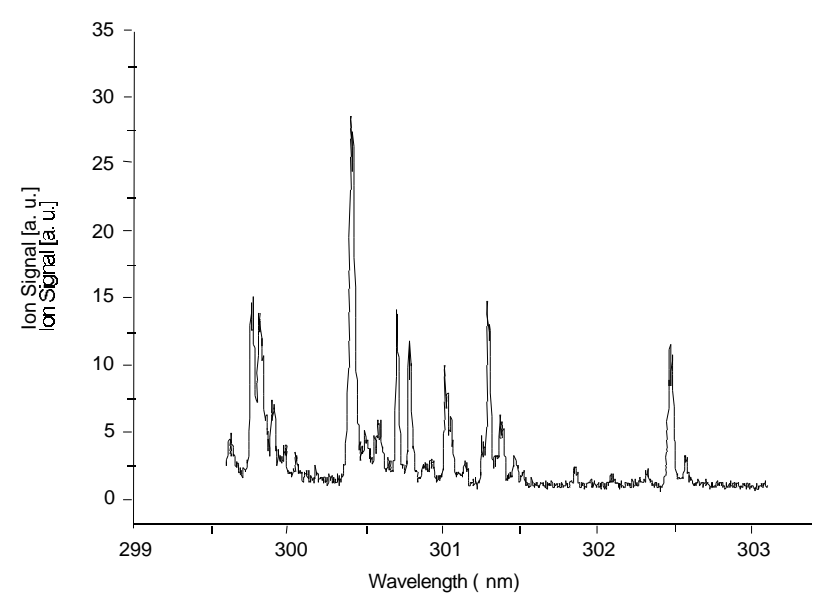

Fig. 2: Wavelength dependence of the $\mathrm{m} / \mathrm{z} 202$ ion signal for jet-REMPI detection of 2-monochlorodihenzofuran
However, in certain cases, using a second color is preferable or essential. Specifically, if the excited state lies at an energy less than half the ionization potential then a second photon of the same wavelength will not have enough energy to ionize the molecule. For example, in tetrachlorinated dioxins (TCDD), the energy of the $S_{1}$ level is approximately half the ionization potential $^{7}$. Thus, singlecolor REMPI may or may not be effective for TCDD. Two-color REMPI using a second, higher energy laser beam may be a much more efficient technique for TCDD detection. The second beam would not require an additional laser. When using a Nd:YAG pumped OPO, the second color can be the

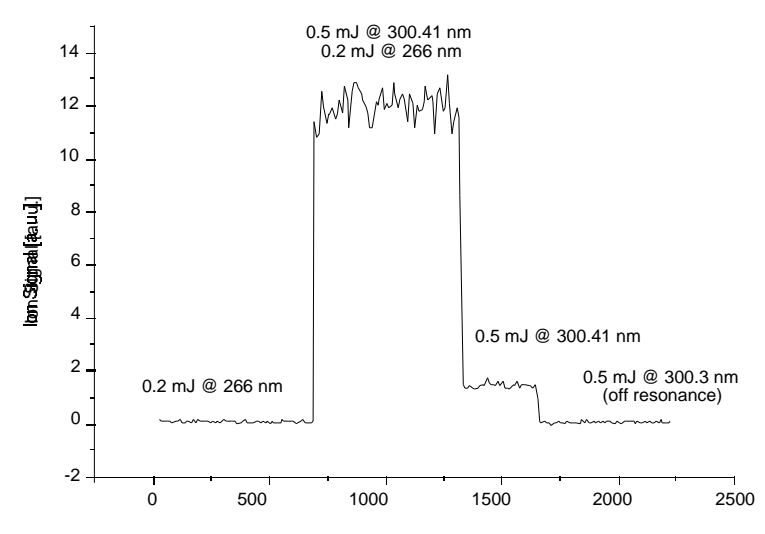

Figure 3: Measured m/z 202 ion signal for jet-REMPI detection of 2-mono-chlorodibenzofuran using various one- and two-color schemes. fourth harmonic at $266 \mathrm{~nm}$ or the fifth harmonic at $213 \mathrm{~nm}$, of the $\mathrm{Nd}: \mathrm{YAG}$ fundamental wavelength produced using nonlinear crystals.

To test this possibility, we reexamined the REMPI signal corresponding to the $\mathrm{m} / \mathrm{z}$ 202 parent ion under a variety of conditions. The result is shown in Figure 3. With only the fixed wavelength (266 $\mathrm{nm})$ light, the signal is at baseline. With the addition of light at $300.41 \mathrm{~nm}$ corresponding to the $\mathrm{S}_{0} \rightarrow \mathrm{S}_{1}$ resonant transition of 2-monochlorofuran, the twocolor REMPI signal appeared and remained constant. Turning off the $266 \mathrm{~nm}$ light caused the ion signal, due now only to the two-photon, one-color REMPI process, to drop by a factor of approximately 8. Detuning the laser off resonance to $300.3 \mathrm{~nm}$ resulted in the signal level again measuring zero. These results appear to confirm the hypothesis that the two-photon, one-color scheme did not impart sufficient energy to achieve a high ionization probability. 


\section{Acknowledgments}

This work was support by the U. S. Department of Energy, National Energy Technology Laboratory, Morgantown, West Virginia under contract DE-AC26-98FT-40370, and the U. S. Environmental Protection Agency under research grant R82792701.

\section{References}

1. Oser, H., Thanner, R., Grotheer, H.-H., Gullett, B. K., Natschke, D., and Raghunathan, K.(1998), Comb. Sci. and Tech., 134, 201.

2. Gullett, B. K., Wikström, E. (2000), Chemosphere, 401015.

3. Zimmermann, R., Heger, H. J., Blumenstock, M., Dorfner, R., Schramm, K. W., Boesl, U., Kettrup, A. (1999), Rapid Comm. Mass Spectr, 13(5), 307.

4. $\quad$ Thanner, R.; Oser, H.; Grotheer, H.-H. (1998), Eur. Mass Spectrom., 4(3), 215.

5. Oser, H.; Coggiola, M. J.; Faris, G. W.; Young, S. E.; Volquardsen, B.; Crosley, R. D. (2001), Applied Optics, 40, 1.

6. Weickhardt, C., Zimmermann, R., Schramm, K.-W., Boesl, U., and Schlag, E. W. (1993), Rapid Comm. Mass. Spectr., 7, 183.

7. Zimmermann, R., Boesl, U., Lenoir, D., Kettrup, A., Grebner, Th. L., Neusser, H. J. (1995), Int. J. Mass. Spectrom. And Ion Process., 145, 97. 